\title{
The Dynamics of \\ Highly Vibrationally Excited CO Scattered from Metal Surfaces
}

\author{
Dissertation
}

zur Erlangung des mathematisch-naturwissenschaftlichen Doktorgrades

„Doctor rerum naturalium“

der Georg-August-Universität Göttingen

im Promotionsprogramm Chemie

der Georg-August University School of Science (GAUSS)

vorgelegt von

Roman Jonathan Viktor Wagner

aus Göttingen

Göttingen, 2019 


\section{$\underline{\text { Betreuungsausschuss: }}$}

Prof. Dr. Alec M. Wodtke

Institut für Physikalische Chemie, Georg-August-Universität Göttingen

Prof. Dr. Dirk Schwarzer

Max-Planck-Institut für Biophysikalische Chemie, Göttingen

\section{Mitglieder der Prüfungskommission:}

Referent:

Prof. Dr. Alec M. Wodtke

Institut für Physikalische Chemie, Georg-August-Universität Göttingen

Korreferent:

Prof. Dr. Dirk Schwarzer

Max-Planck-Institut für Biophysikalische Chemie, Göttingen

Weitere Mitglieder der Prüfungskommission:

Prof. Dr. Jörg Behler

Institut für Physikalische Chemie, Georg-August-Universität Göttingen

Prof. Dr. Burkhard Geil

Institut für Physikalische Chemie, Georg-August-Universität Göttingen

Prof. Dr. Theofanis N. Kitsopoulos

Max-Planck-Institut für Biophysikalische Chemie, Göttingen

Prof. Dr. Jürgen Troe

Institut für Physikalische Chemie, Georg-August-Universität Göttingen

Tag der mündlichen Prüfung: 09.04.2019 


\section{Kurzfassung}

Ein Überschallmolekülstrahl aus hochschwingungsangeregten CO Molekülen wird von $\mathrm{Au}(111)$ und $\mathrm{Ag}(111)$ Oberflächen gestreut. Dafür werden die einfallenden CO Moleküle in dem $\mathrm{X}^{1} \Sigma^{+}(v=17, J=0)$ Zustand präpariert. Die gestreuten Moleküle werden mittels resonanzverstärkter Multiphotonenionisation quantenzustandsspezifisch detektiert. Es werden sowohl Schwingungszustands- und Rotationszustandsverteilungen als auch Winkel- und Geschwindigkeitsverteilungen der gestreuten Moleküle präsentiert. Hochrotationsangeregte Streuprodukte, schmale Winkelverteilungen, und finale Translationsenergien, die in Übereinstimmung mit dem Baule-Grenzwert sind, lassen auf einen direkten Streumechanismus schließen. Die Wahrscheinlichkeit, dass $\operatorname{CO}(v=17)$ einen oder mehrere Schwingungsquanten verliert, ist für Kollisionen mit $\mathrm{Ag}(111)$ höher als für $\mathrm{Au}(111)$. Für beide Metalle wird eine Zunahme der Relaxationswahrscheinlichkeit mit erhöhter Translationsenergie der einfallenden Moleküle beobachtet. Diese Abhängigkeit ist für $\mathrm{Au}(111)$ stärker ausgeprägt als für $\mathrm{Ag}(111)$. Ein umfassender Vergleich mit Schwingungsrelaxationswahrscheinlichkeiten anderer Molekül-OberflächenSysteme diese umfassen zwei Moleküle (CO und NO), zwei Oberflächen (Gold und Silber) und eine Vielzahl unterschiedlicher Schwingungsanregungen der einfallenden Moleküle $(2 \leq v \leq 17)$-zeigt einen einheitlichen Trend auf, demzufolge die Relaxationswahrscheinlichkeit sowohl von der Austrittsarbeit der Oberfläche als auch von der Elektronenbindungsenergie des Moleküls abhängt. Dies ist ein starker Hinweis darauf, dass die elektronisch nichtadiabatische Wechselwirkung zwischen der Schwingung des Moleküls und der elektronischen Anregung der Oberfläche durch einen Elektronentransferprozess vermittelt wird. Die Relaxationswahrscheinlichkeit schwingungsangeregter Moleküle in einfachen Zusammenstößen mit Festkörperoberflächen kann demnach auf Grundlage der energetischen Eigenschaften des isolierten Moleküls und der isolierten Oberfläche vorhergesagt werden. Außerdem können durch Betrachtung der Austrittsarbeit und der Elektronenbindungsenergie des Moleküls jene Molekül-Oberflächen-Systeme identifiziert werden, deren elektronisch nichtadiabatische Dynamik an Oberflächen vornehmlich durch einen Elektronentransfer zwischen Oberfläche und Molekül bestimmt wird. 


\section{Abstract}

Supersonic molecular beams of highly vibrationally excited CO are scattered from atomically clean $\mathrm{Au}(111)$ and $\mathrm{Ag}(111)$ surfaces. Specifically, incident $\mathrm{CO}$ is prepared in the $\mathrm{X}^{1} \Sigma^{+}(v=17, J=0)$ state. Scattered molecules are detected quantum state-selectively by means of resonance-enhanced multi-photon ionization. Final vibrational state distributions and rotational state distributions are presented as well as time-of-flight distributions and angular distributions. Rotationally hot scattering products, narrow angular distributions, and final translational energies consistent with the Baule limit indicate a direct scattering mechanism. The vibrational relaxation probability of $\mathrm{CO}(v=17)$ at $\mathrm{Ag}(111)$ is higher than that at $\mathrm{Au}(111)$. For both metals, the vibrational relaxation probability increases with incidence translational energy. The incidence translational energy dependence is more pronounced for $\mathrm{Au}(111)$ than for $\mathrm{Ag}(111)$. The comparison to previously studied molecule-surface systems - including two molecules (CO and NO), two surfaces (gold and silver), and various incidence vibrational excitations $(2 \leq v \leq 17)$ - reveals a unifying trend, according to which the vibrational relaxation probability depends on both the work function of the surface and the electron binding energy of the molecule. This strongly suggests that an electron transfer process is essential to the electronically non-adiabatic coupling between molecular vibration and electronic degrees of freedom of the surface. Thus, for a single-bounce collision event with a metal surface, the vibrational relaxation probability of a diatomic molecule can be predicted simply by evaluating energetic quantities characterizing the isolated molecule and surface. This allows easy identification of molecule-surface systems in which non-adiabatic surface dynamics are likely to be governed by electron transfer. 


\section{Contents}

$\begin{array}{lr}\text { Kurzfassung } & 3\end{array}$

$\begin{array}{ll}\text { Abstract } & 5\end{array}$

1 Introduction 9

2 Theoretical Background $\quad 17$

2.1 Electronic Non-Adiabaticity . . . . . . . . . . . . . . . . . . . . . . . 17

2.1 .1 Introduction . . . . . . . . . . . . . . 17

2.1.2 Electronically Non-Adiabatic Dynamics at Surfaces . . . . . . . . 23

2.2 Electronic Spectroscopy of Carbon Monoxide . . . . . . . . . . . . . 26

2.3 Stark Deceleration of Molecular Beams . . . . . . . . . . . . . . . . 31

3 Experimental $\mathbf{3 7}$

3.1 Beam-Surface Scattering Apparatus . . . . . . . . . . . . . . . 37

3.2 The Stark Machine . . . . . . . . . . . . . . . . . . . . . . . . 41

3.3 Laser Systems . . . . . . . . . . . . . . . . . . . . . . . . 47

3.4 Experimental Procedures . . . . . . . . . . . . . . . . 49

3.4.1 Preparation and Detection of $\mathrm{CO} \mathrm{X}^{1} \Sigma^{+}(v=17) \ldots \ldots . \ldots 49$

3.4.2 Detection of $\mathrm{CO} \mathrm{a}^{3} \Pi_{1}(v=0) \ldots \ldots \ldots \ldots . \ldots \ldots$

3.4.3 Preparation of Chlorinated $\mathrm{Ag}(111) \ldots \ldots \ldots \ldots$

3.4 .4 Surface Scattering Experiments . . . . . . . . . . . . . . . 58

3.5 Chemicals . . . . . . . . . . . . . . . . . . 60

4 Data Analysis $\quad 61$

4.1 Time-of-Flight Distributions . . . . . . . . . . . . . . . . . . . . . . . . . . . . . 61

4.2 Angular Distributions . . . . . . . . . . . . . . . . . . 64

4.3 Vibrational State Distributions . . . . . . . . . . . . . . . . 66

4.3.1 Relative Vibrational Populations . . . . . . . . . . . . . . 66

4.3.2 Vibrational Relaxation Probabilities . . . . . . . . . . . . 70

4.4 Rotational State Distributions . . . . . . . . . . . . . . . . . 72 
Contents

5 Surface Scattering of $\mathrm{CO} \mathrm{X}^{1} \Sigma^{+}(v=17) \quad 77$

5.1 Time-of-Flight Distributions . . . . . . . . . . . . . . . . . . 77

5.1 .1 Results . . . . . . . . . . . . . . . 77

5.1 .2 Discussion: Translational Inelasticity . . . . . . . . . . . . . 82

5.2 Angular Distributions . . . . . . . . . . . . . . . . . . 92

5.3 Vibrational State Distributions _... . . . . . . . . . . . 94

5.3 .1 Results . . . . . . . . . . . . . . . . . 95

5.3.2 Vibrational Relaxation Mechanism . . . . . . . . . . . . . . . 102

5.3.3 Discussion: Vibrational Inelasticity . . . . . . . . . . . . . . . . . 109

5.4 Rotational State Distributions . . . . . . . . . . . . . . . . . 117

6 Towards Stark-Decelerated Beams of $\mathrm{CO} \mathrm{X}^{1} \Sigma^{+}(v=17) \quad 121$

6.1 Motivation . . . . . . . . . . . . . . . . 121

6.2 Approaches . . . . . . . . . . . . . . . . . . . 122

6.2 .1 Velocity Scans . . . . . . . . . . . . . . . 122

6.2.2 Preparation of Stark-decelerated CO X ${ }^{1} \Sigma^{+}(v=17) \quad \ldots \ldots . .124$

6.2.3 Spatial Separation of $\mathrm{CO} \mathrm{X}^{1} \Sigma^{+}$and $\mathrm{CO}^{3} \Pi_{1} \ldots \ldots \ldots 128$

$\begin{array}{lll}7 & \text { Conclusion } & 133\end{array}$

$\begin{array}{ll}\text { A Appendix } & \mathbf{1 3 7}\end{array}$

A.1 Rotational Transition Wavenumbers of the $\mathrm{A}^{1} \Pi-\mathrm{X}^{1} \Sigma^{+}$System in CO . 137

$\begin{array}{ll}\text { List of Abbreviations } & 142\end{array}$

$\begin{array}{ll}\text { Bibliography } & 143\end{array}$

$\begin{array}{ll}\text { Acknowledgments } & 161\end{array}$

$\begin{array}{ll}\text { List of Publications } & 163\end{array}$ 


\section{Introduction}

Simply put, the present work is about playing squash on the atomic scale. A molecular beam consistent of carbon monoxide molecules is hit against a metallic wall and the energetic states of the molecules are probed before and after the collision. This way, energy transfer processes at the gas-solid interface can be investigated experimentally. Accordingly, the present work constitutes a contribution to the field of chemical dynamics at surfaces.

What is the field of chemical dynamics at surfaces all about? In general, the surface of an object represents the object's outermost layer. From a chemist's point of view, surfaces occur at the interfaces between different phases. The solid phase, the liquid phase, and the gaseous phase, for instance, are states of matter that are generally known from everyday experience. Thus, it is not surprising that surfaces and chemical processes at surfaces are ubiquitous. A well-known example of such a process is the corrosion of iron in the presence of water and air, a chemical reaction that nearly everyone has to deal with at some point. From a microscopic point of view, the formation of rust is a complex interplay between molecules and atoms that occur as gaseous oxygen, liquid water, and solid iron. In the field of chemical dynamics at surfaces, chemists and physicists are aiming at a fundamental understanding of such surface-chemical processes.-Dynamics means the movement of, the forces acting on, and the energy transferred between atoms and molecules. Chemical reaction means the cleavage and formation of bonds between atoms during which reactant molecules are consumed and product molecules are formed.

At an early stage in the history of surface science [1], Johann Wolfgang Döbereiner reported in 1823 [2] "that finely divided platinum powder has the astonishing ability to convert hydrogen and oxygen to water by mere contact and without any external influences." ${ }^{2}$ This observation led to the invention of Döbereiner's lamp, which was a commercially available lighter that initiates a flame by exposing a sponge of platinum to a mixture of air and hydrogen gas [3]. Here, the surface of the platinum serves as a

\footnotetext{
1“[...] daß das rein metallische staubförmige Platin die höchst merkwürdige Eigenschaft hat, das Wasserstoffgas durch bloße Berührung und ohne alle Mitwirkung äußerer Potenzen zu bestimmen, daß es sich mit Sauerstoffgas zu Wasser verbindet [...]." Correspondence between Johann Wolfgang Döbereiner and Johann Wolfgang von Goethe in July 1823 [2].
} 


\section{Introduction}

catalytic agent that reduces the activation energy of the combustion reaction such that the gas mixture ignites spontaneously. In 1902, Wilhelm Ostwald defined a catalyst as "a substance which alters the rate of a chemical reaction without being part of the final reaction products." ${ }^{2}$ [4] Since then, the field of heterogeneous catalysis - the type of catalysis in which the phase of the reactants differs from that of the catalyst - evolved rapidly to one of the most important fields in surface science [5].

Nowadays, heterogeneous catalysis is crucial to many industrial applications or processes found in everyday life. The catalytic converter in a car, for instance, catalyzes the oxidation of poisonous $\mathrm{CO}$ to $\mathrm{CO}_{2}$ before the gas leaves the exhaust pipe. In large-scale industrial applications, the majority of chemical reactions involve the use of a heterogeneous catalyst, for instance in the production of propargyl alcohol out of acetylene and formaldehyde, in the hydrogenation of butynediol to form butanediol, in the HaberBosch synthesis of ammonia out of nitrogen and hydrogen, in the steam reforming of natural gas to produce hydrogen $\left(\mathrm{CH}_{4}+\mathrm{H}_{2} \mathrm{O} \rightleftharpoons \mathrm{CO}+3 \mathrm{H}_{2}\right)$, or in the water-gas shift reaction $\left(\mathrm{CO}+\mathrm{H}_{2} \mathrm{O} \rightleftharpoons \mathrm{CO}_{2}+\mathrm{H}_{2}\right)$, just to mention a few. Even though the drastic reduction of energy needed to run such chemical reactions is a great advantage, one should not forget that heterogeneous catalysis often involves the use of transition metals or related compounds that might be harmful to the environment.

In order to improve surface chemical reactions, the effect of pressure, temperature, or composition on the reaction yield can be studied. However, this approach is limited when it comes to large-scale applications because small test reactors are needed which are possibly incapable of reproducing reaction conditions present in large-scale reactors. As early as 1922, a fundamentally different approach was formulated by Irving Langmuir [6], who was working in Göttingen at one of the first German institutes of physical chemistry. Langmuir says, "most finely divided catalysts, such as platinum black, or activated charcoal, etc., must have structures of great complexity [...]. In order to simplify our theoretical consideration of reactions on surfaces, let us confine our attention mainly to reactions on plain surfaces. If the principles in this case are well understood, it should then be possible to extend the theory to the case of porous bodies." ${ }^{3}$ Langmuir's approach aims at a fundamental understanding of elementary processes in surface chemical reactions gained by studying model systems with reduced complexity. This forms the cornerstone of a large field of fundamental research in surface chemistry which comes along with the development of new strategies and methods.

The advent of new technologies around the 1960s unleashed the full potential of sur-

\footnotetext{
2 "Ein Katalysator ist jeder Stoff, der, ohne im Endprodukt einer chemischen Reaktion zu erscheinen, ihre Geschwindigkeit verändert." Page 316 in Reference [4].

${ }^{3}$ Page 616 in Reference [6].
} 
face science as it is known in its present form, still following the approach proposed by Langmuir. One of the most important pioneers in the field of modern surface science is Gerhard Ertl [7], who received the Nobel Prize in Chemistry for his studies of chemical processes on solid surfaces [1]. For example, he developed new methods to study the catalytic synthesis of ammonia from the elements on iron and identified all surface intermediates and their kinetics. In general, experimental setups designed and built to study surface chemical reactions on the atomic scale combine a number of different technologies, such as ultra-high vacuum (UHV) generation, rare gas ion sputtering, supersonic molecular beam production, metal single crystal growth, and laser based light sources. A number of surface sensitive methods and spectroscopic tools emerged, for instance Auger electron spectroscopy (AES), low-energy electron diffraction (LEED), X-ray and ultraviolet photoelectron spectroscopy (XPS and UPS), scanning tunneling microscopy (STM), and atomic force microscopy (AFM). Thus, a large number of tools is available to study elementary processes in surface chemistry. Studies of this kind address the following questions. Is an atom or molecule that collides with a surface directly scattered off of the surface or does it stick to the surface? If it sticks, is it physisorbed or chemisorbed? What is the fate of the projectile's incident translational energy if it sticks? Does an adsorbed molecule dissociate on the surface or does it stay intact? What are the underlying principles of dissociative adsorption and associative desorption? To which surface site does a molecule bind? Does an adsorbed molecule react with co-adsorbates preferentially on flat terraces or at atomic steps and kinks? What are possible dissipation channels for energy released in chemical reactions at surfaces? Does a molecule that directly scatters off of the surface gain or lose translational energy, vibrational energy, or rotational energy? Do molecular degrees of freedom (like vibration, rotation, and translation) couple to surface degrees of freedom (like phonons and electron-hole pairs)? By which mechanism is energy transferred between different degrees of freedom? Which degree of freedom needs to be excited in order to drive a chemical reaction along a certain reaction coordinate?

Surface chemistry evolved to a versatile field having impact on various applications and technologies [8]. Current trends in fundamental research in this field are reviewed in a selection of articles recently published in the journal Chemical Society Reviews, which dedicated a whole issue to the topic "Surface Reaction Dynamics" [9]. Skimming over the headlines of these articles reveals the equivalent importance of experimental and theoretical investigations in this field. It is the fruitful collaboration between experimentalists and theorists that pushes the limits of our current understanding of surface-dynamical processes.

Theorists are confronted with the challenge of predicting the outcome of complex 


\section{Introduction}

surface-dynamical processes using ab initio methods. In 1931, Henry Eyring and Michael Polanyi introduced the standard model of chemical reactivity in order to predict the dynamics of chemical systems $[10,11]$. Basically, their approach is twofold. First, a potential energy surface (PES) is constructed by calculating the potential energy of a chemical system for different geometric arrangements. Second, the PES is used to calculate the dynamics of the system, which includes the movement of particles and the energy exchanged between them. The standard model of chemical reactivity is based on the Born-Oppenheimer approximation (BOA) [12], which drastically simplifies the solutions of the Schrödinger equation for many-body problems by parametrically separating the electronic motion from the nuclear motion. Based on the large mass mismatch between electrons and nuclei, the Born-Oppenheimer approximation-also known as the adiabatic approximation - assumes that the electronic configuration of a system readjusts instantaneously to a change in the nuclear configuration. As a consequence, dynamics on an adiabatic PES exclude electronic excitations induced by nuclear motion.

The importance of accurate PES in understanding chemical reactivity cannot be overstated [13]. For molecule-molecule interactions, theoretical models attain such a high level of accuracy that the comparison to experiment requires the use of advanced experimental techniques such as velocity-map imaging (VMI) [14, 15], Rydberg atom tagging (RAT) [16], or Stark deceleration of molecular beams [17, 18]. For example, crossed-molecular beam experiments taking advantage of the state selectivity and the velocity control of Stark-decelerated molecular beams impressively reveal the quantum nature of molecules by showing diffraction oscillations in the scattering of NO from rare gas atoms, which is in good agreement with theory [19].

Indeed, many molecule-molecule interactions can be described within the adiabatic picture. However, there are cases for which transitions between adiabatic potential energy surfaces - so-called electronically non-adiabatic transitions - need to be taken into account. For example, this has been recently discussed for the vibrational relaxation of $\mathrm{NO}(v=1)$ in collisions with argon atoms [20]. In contrast to molecule-atom or moleculemolecule interactions, electronically non-adiabatic effects may play a more pronounced role in molecule-surface interactions, especially in the interaction of molecules with metal surfaces. Due to the continuum of electronic states in the metal's conduction band, adiabatic states of a molecule-surface system can be very close in energy, which facilitates non-adiabatic hops between those states. It is thus reasonable to question the validity of the Born-Oppenheimer approximation when modeling molecule-surface interactions [21, 22]. Accordingly, the study of electronically non-adiabatic dynamics at surfaces evolved to an important field in surface science [23]. 
Electronically non-adiabatic dynamics at surfaces are characterized by a coupling of the nuclear motion of the molecule with electronic degrees of freedom of the surface. Thus, non-adiabatic effects may occur in a number of processes such as the vibrational relaxation of adsorbates, the vibrational excitation or relaxation of molecules in direct surface-scattering events, or the dissipation of energy released during the adsorption or reaction of molecules at surfaces. For instance, the scattering of $\mathrm{H}$ atoms from $\mathrm{Au}(111)$ shows that the translational energy loss needed to make an $\mathrm{H}$ atom stick to a metal surface relies on electron-hole pair (EHP) excitation in the metal [24]. The $\mathrm{H} / \mathrm{Au}(111)$ system represents one of the most fundamental atom-metal systems one can possibly think of. Furthermore, the scattering of highly vibrationally excited $\operatorname{NO}(v=15)$ from $\mathrm{Au}(111)$ indicates that the vibrational relaxation during the molecule-surface encounter is governed by an electron transfer from the metal to the molecule [25]. The $\mathrm{NO} / \mathrm{Au}(111)$ system serves as a benchmark system for non-adiabatic energy transfer at the gas-solid interface as it is one of the most extensively studied molecule-metal systems.

Theorists put a lot of effort into constructing accurate molecule-metal interaction potentials in order to model non-adiabatic dynamics at surfaces. Density functional theory (DFT) is most commonly used in order to deal with the large number of atoms in molecule-surface systems. Electronic friction (EF) based [26, 27] and independentelectron surface hopping (IESH) based [28, 29] approaches have been developed to treat the Born-Oppenheimer approximation breakdown in molecule-surface interactions. The physical pictures of these approaches are fundamentally different. While EF describes the motion of nuclei on a single effective PES and accounts for non-adiabatic effects by means of frictional forces acting on the nuclei, IESH employs a multistate PES and describes electron-hole pair excitations by hops between electronic adiabats. When it comes to surface-scattering of vibrationally excited molecules, the comparison to experiment shows that neither approach is yet capable of predicting the correct final vibrational state distribution or the correct dependence on incidence parameters [30]. Further work by theorists and experimentalists is necessary to understand the mechanistic details relevant to the vibrational relaxation of molecules at surfaces.

The present thesis is an experimental study on the vibrational relaxation of diatomic molecules during single-bounce collisions with well-defined metal surfaces. The main part of the present work focuses on the scattering of highly vibrationally excited $\mathrm{CO}$ $\mathrm{X}^{1} \Sigma^{+}(v=17, J=0)$ from $\mathrm{Au}(111)$ and $\mathrm{Ag}(111)$, as sketched in Figure 1.1. Scattering experiments include the measurement of final vibrational state distributions, final rotational state distributions, angular distributions, and time-of-flight distributions probing final translational energies. Throughout the experiments, the incidence translational energy is varied between $0.27 \mathrm{eV}$ and $0.57 \mathrm{eV}$. Major parts of results presented in this 


\section{Introduction}

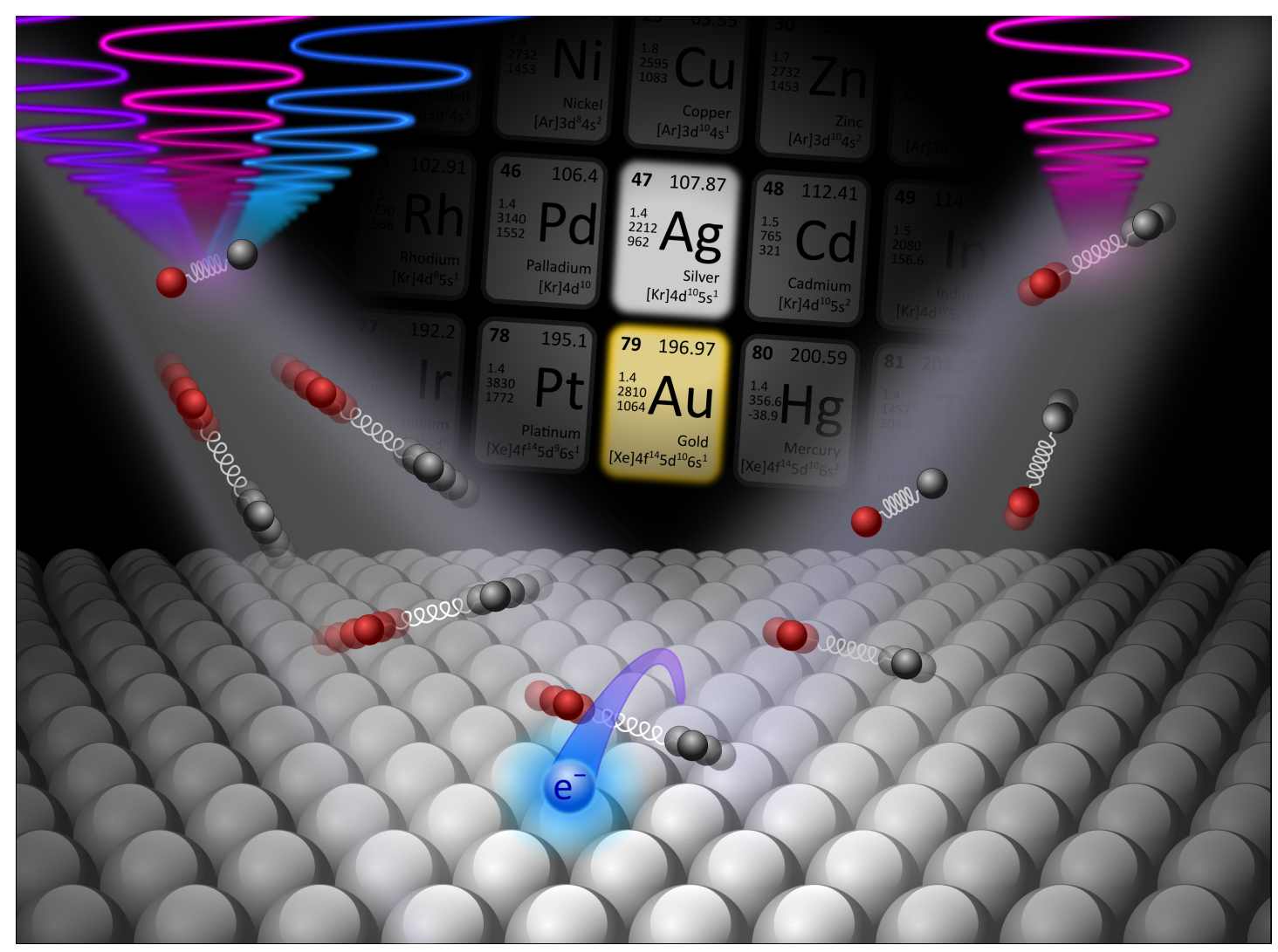

Figure 1.1: Electron transfer mediated vibrational relaxation of diatomic molecules at metal surfaces. Three-laser excitation prepares incident $\mathrm{CO}$ in a highly vibrationally excited state. Vibrationally excited $\mathrm{CO}$ is scattered from an atomically flat silver or gold surface. Scattered molecules are laserdetected by means of resonance-enhanced multi-photon ionization. The figure is adapted from the front cover published in Physical Chemistry Chemical Physics, Volume 21, Issue 4 [32].

thesis, primarily on vibrationally inelastic scattering, are published in peer-reviewed journals $[31,32]$. A smaller part of the thesis describes attempts that have been made to utilize Stark-decelerated molecular beams in beam-surface scattering experiments.

Even though a little exotic, highly vibrationally excited molecules are excellent candidates for the investigation of electronic non-adiabaticity in molecule-surface interactions because they are likely to exhibit a strong vibration-EHP coupling [25]. So far, surfacedynamical effects of highly vibrationally excited diatomic molecules have been studied only for the NO molecule. Thus, results presented for highly vibrationally excited CO 
represent an important extension to existing benchmark data. Together with previously reported results, the results obtained for $\mathrm{CO}(v=17) / \mathrm{Au}(111)$ and $\mathrm{CO}(v=17) / \mathrm{Ag}(111)$ complete a comprehensive data set which contains surface-dynamical information on various molecule-surface systems, including two molecules (CO and NO), two surfaces (gold and silver), and different initial vibrational excitations $(2 \leq v \leq 17)$. The comparison of these systems confirm a unifying trend which has been proposed earlier [33, 34]: The non-adiabatic propensity of a certain molecule-surface system increases with decreasing surface work function and with increasing electron binding energy of the molecule. Now, a sufficient amount of data is available to observe this trend when comparing vibrational relaxation probabilities in different systems. This observation strongly suggests that the vibrational relaxation of diatomic molecules at metal surfaces is mediated by an electron transfer process.

The present work is structured as follows. Chapter 2 provides an introduction to electronic non-adiabaticity in general and to electronic non-adiabaticity in molecule-surface interactions. Furthermore, the reader is introduced to the concept of Stark deceleration, and to the energetic structure of the $\mathrm{CO}$ molecule, which is relevant to spectroscopic methods mentioned in Chapter 3. Chapter 3 describes experimental setups and procedures, including beam-surface scattering apparatuses and spectroscopic methods used to prepare and detect certain quantum states of CO. Chapter 4 explains the analysis that is performed on the raw data in order to yield vibrational state distributions, rotational state distributions, angular distributions, and translational energies. Results obtained for the scattering of $\mathrm{CO}(v=17)$ from $\mathrm{Au}(111)$ and $\mathrm{Ag}(111)$ are presented and discussed in Chapter 5. Chapter 6 describes the attempts that have been made to use Stark-decelerated molecular beams in surface scattering experiments. Concluding remarks are made in Chapter 7. 



\section{Theoretical Background}

\subsection{Electronic Non-Adiabaticity}

\subsubsection{Introduction}

The word adiabatic stems from the Greek word adiabatos ( $\alpha \dot{\delta} \iota \dot{\alpha} \beta \alpha \tau o s)$, meaning "not

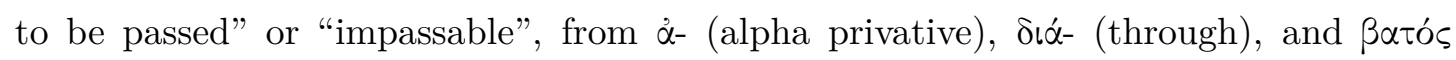
(passable, accessible) [35]. A thermodynamic system is called adiabatic when it cannot exchange heat with its surroundings. As heat cannot pass the border between the system and its surroundings, energy exchange is only possible by means of work. In the field of chemical reaction dynamics, however, a process is called adiabatic if the reaction occurs on a single adiabatic potential energy surface (PES), meaning that the system cannot pass the gap between two close-by PESs. In the adiabatic approximation, the electronic wave function evolves from the reactant electronic configuration to the product electronic configuration on a time scale that is fast compared to the movement of nuclei. If, however, the instantaneous rearrangement of the electronic configuration is not possible, the system hops from one adiabatic PES to another one and ends up in a different electronic state. These hops are designated as non-adiabatic transitions. In the following, the meaning of adiabaticity and non-adiabaticity is elucidated in more detail by introducing the reader to concepts such as the Born-Oppenheimer approximation (BOA) and potential energy surfaces. Electronic non-adiabaticity in chemical reaction dynamics has been reviewed in a number of articles [23, 36-40], which form the basis of the present section.

In the 1920s, the newly developed quantum theory provided a fundamentally new understanding of the structure of atoms. For the lightest atom, the hydrogen atom, quantum theory allows the exact computation of electronic energies and transition frequencies between electronic states. As the complexity increases dramatically with the number of interacting nuclei and electrons, exact numerical calculations are not possible for larger systems such as heavier atoms and molecules. Nevertheless, Born and Oppenheimer realized in 1927 that the lighter electrons move much faster than the heavier nuclei. They concluded, to a good approximation, that the electronic configuration of 


\section{Theoretical Background}

a system readjusts instantaneously to a change in the nuclear configuration [12]. By separating nuclear and electronic motion, the electronic energy of a system can be calculated for a fixed nuclear configuration. By repeating this calculation for different nuclear arrangements, a multidimensional map of electronic energies is obtained as a function of nuclear coordinates, the so-called potential energy surface. Based on the Born-Oppenheimer approximation, Eyring and Polanyi established the standard model of chemical reactivity in 1931 by using potential energy surfaces to predict the reaction dynamics of chemical systems $[10,11]$. According to this model, a reacting system evolves from the reactants to the products on a single PES in an electronically adiabatic way. Since the model makes direct use of the Born-Oppenheimer approximation, it is worthwhile to discuss the Born-Oppenheimer approximation in more detail in order to elucidate the meaning of electronic adiabaticity. This requires a short mathematical digression, which adapts the notation used by Tully [39, 40].

According to the first postulate of quantum mechanics, the properties of a certain state $i$ of a quantum mechanical system are entirely specified by the wave function $\Psi_{i}(\boldsymbol{r}, \boldsymbol{R})$. The wave function contains information about the energy of the system as a function of electronic coordinates $\boldsymbol{r}$ and nuclear coordinates $\boldsymbol{R}$. Energy eigenvalues $E_{i}$ can be determined by solving the time-independent Schrödinger equation, in which the Hamiltonian operator $\hat{H}$ acts on the wave function.

$$
\hat{H}\left|\Psi_{i}(\boldsymbol{r}, \boldsymbol{R})\right\rangle=E_{i}\left|\Psi_{i}(\boldsymbol{r}, \boldsymbol{R})\right\rangle
$$

Without considering spin-orbit interactions, the full non-relativistic molecular Hamiltonian operator can be written as

$$
\hat{H}=\hat{T}_{\mathrm{N}}+\hat{T}_{\mathrm{e}}+\hat{V}_{\mathrm{e}}+\hat{V}_{\mathrm{eN}}+\hat{V}_{\mathrm{N}},
$$

where $\hat{T}_{\mathrm{N}}$ and $\hat{T}_{\mathrm{e}}$ describe the nuclear and electronic kinetic energy, and $\hat{V}_{\mathrm{e}}, \hat{V}_{\mathrm{eN}}$, and $\hat{V}_{\mathrm{N}}$ describe the electron-electron, electron-nucleus, and nucleus-nucleus Coulomb interactions, respectively.

$$
\begin{gathered}
\hat{T}_{\mathrm{N}}=\sum_{M=1}^{N-1}-\frac{\hbar^{2}}{2 \mu_{M}} \nabla_{M}^{2} \\
\hat{T}_{\mathrm{e}}=\sum_{k=1}^{l}-\frac{1}{2} \hbar^{2} \nabla_{k}^{2} \\
\hat{V}_{\mathrm{e}}=\sum_{k=1}^{l-1} \sum_{k^{\prime}>1}^{l} \frac{e^{2}}{\left|\boldsymbol{r}_{k}-\boldsymbol{r}_{k^{\prime}}\right|} \\
\hat{V}_{\mathrm{eN}}=-\sum_{k=1}^{l} \sum_{M=1}^{N} \frac{z_{M} e^{2}}{\left|\boldsymbol{r}_{k}-\boldsymbol{R}_{M}\right|}
\end{gathered}
$$




$$
\hat{V}_{\mathrm{N}}=\sum_{M=1}^{N-1} \sum_{M^{\prime}>M}^{N} \frac{z_{M} z_{M^{\prime}} e^{2}}{\left|\boldsymbol{R}_{M}-\boldsymbol{R}_{M^{\prime}}\right|}
$$

$M$ and $k$ are indices for the nuclei and electrons, and $N$ and $l$ denote the total number of nuclei and electrons, respectively. $\mu_{M}$ is the reduced mass, $\hbar$ is Planck's constant, $e$ is the elementary charge, and $z_{M}$ is the charge associated with nucleus $M$.

Exploiting the large difference between the electronic and nuclear masses, the electronic motion can be separated from the nuclear motion by expressing $\Psi_{i}(\boldsymbol{r}, \boldsymbol{R})$ in terms of an electronic basis function $\phi_{i}(\boldsymbol{r} ; \boldsymbol{R})$ and a nuclear basis function $\chi_{i}(\boldsymbol{R})$.

$$
\Psi_{i}(\boldsymbol{r}, \boldsymbol{R})=\phi_{i}(\boldsymbol{r} ; \boldsymbol{R}) \chi_{i}(\boldsymbol{R})
$$

Electronic basis functions are chosen to be adiabatic, meaning that they depend parametrically on $\boldsymbol{R}$. The parametric dependence is indicated by the semicolon. The electronic part of the Schrödinger equation (see Equation 2.10) can be solved for a given set of stationary nuclear coordinates. The electronic wave functions $\phi_{i}(\boldsymbol{r} ; \boldsymbol{R})$ need to vary continuously with $\boldsymbol{R}$ and form an orthonormal set for every value of $\boldsymbol{R}$. The nuclear wave functions $\chi_{i}(\boldsymbol{R})$ describe the motion of nuclei on the potential energy surface of a certain electronic state $i$.

The electronic Hamiltonian $\hat{H}_{\mathrm{el}}$ for fixed values of $\boldsymbol{R}$ is usually defined as

$$
\hat{H}_{\mathrm{el}}=\hat{T}_{\mathrm{e}}+\hat{V}_{\mathrm{e}}+\hat{V}_{\mathrm{eN}}+\hat{V}_{\mathrm{N}}
$$

In the adiabatic representation, the electronic basis functions are eigenfunctions of $\hat{H}_{\text {el }}$.

$$
\hat{H}_{\mathrm{el}}\left|\phi_{i}(\boldsymbol{r} ; \boldsymbol{R})\right\rangle=U_{i}(\boldsymbol{R})\left|\phi_{i}(\boldsymbol{r} ; \boldsymbol{R})\right\rangle
$$

$U_{i}(\boldsymbol{R})$ is the eigenvalue of $\hat{H}_{\mathrm{el}}$ for a certain electronic state $i$. Plugging Equations 2.8, 2.9 , and 2.10 into 2.1 yields

$$
\begin{aligned}
\hat{H}\left|\phi_{i}(\boldsymbol{r} ; \boldsymbol{R}) \chi_{i}(\boldsymbol{R})\right\rangle= & \left(\hat{T}_{\mathrm{N}}+\hat{H}_{\mathrm{el}}\right)\left|\phi_{i}(\boldsymbol{r} ; \boldsymbol{R}) \chi_{i}(\boldsymbol{R})\right\rangle \\
= & \hat{T}_{\mathrm{N}}\left|\phi_{i}(\boldsymbol{r} ; \boldsymbol{R}) \chi_{i}(\boldsymbol{R})\right\rangle+U_{i}(\boldsymbol{R})\left|\phi_{i}(\boldsymbol{r} ; \boldsymbol{R}) \chi_{i}(\boldsymbol{R})\right\rangle \\
= & \sum_{M=1}^{N-1} \frac{-\hbar^{2}}{2 \mu_{M}}\left(\phi_{i}(\boldsymbol{r} ; \boldsymbol{R}) \cdot \nabla_{M}^{2}\left|\chi_{i}(\boldsymbol{R})\right\rangle\right. \\
& +2 \cdot \underbrace{\nabla_{M}\left|\phi_{i}(\boldsymbol{r} ; \boldsymbol{R})\right\rangle}_{\approx 0} \cdot \nabla_{M}\left|\chi_{i}(\boldsymbol{R})\right\rangle \\
& +\chi_{i}(\boldsymbol{R}) \cdot \underbrace{\nabla_{M}^{2}\left|\phi_{i}(\boldsymbol{r} ; \boldsymbol{R})\right\rangle}_{\approx 0}) \\
& +U_{i}(\boldsymbol{R})\left|\phi_{i}(\boldsymbol{r} ; \boldsymbol{R}) \chi_{i}(\boldsymbol{R})\right\rangle
\end{aligned}
$$




\section{Theoretical Background}

According to the Born-Oppenheimer approximation, the electrons adjust instantaneously to a change in nuclear positions and, thus, adjust to the same optimum configuration that they would have if the nuclei were stationary. This excludes transitions between different electronic states induced by nuclear motion. Consequently, terms in which the nuclear operator $\nabla_{M}$ acts on the electronic wave function $\phi_{i}(\boldsymbol{r} ; \boldsymbol{R})$ are neglected, as indicated in Equation 2.13. The consequences of the Born-Oppenheimer approximation become obvious when multiplying the Schrödinger equation from the left by $\phi_{i}^{*}(\boldsymbol{r} ; \boldsymbol{R})$ and integrating over all electronic coordinates $\boldsymbol{r}$, which yields an infinite set of coupled equations for the nuclear wave function $\chi_{i}(\boldsymbol{R})$.

$$
\begin{aligned}
& \sum_{j}^{\infty}\left\langle\phi_{i}(\boldsymbol{r} ; \boldsymbol{R})|\hat{H}| \phi_{j}(\boldsymbol{r} ; \boldsymbol{R})\right\rangle \chi_{j}(\boldsymbol{R}) \\
& =\left(\mathscr{T}_{\mathrm{N}}+\mathscr{T}_{i i}^{\prime \prime}+U_{i i}\right) \chi_{i}(\boldsymbol{R})+\sum_{j \neq i}^{\infty}\left(\mathscr{T}_{i j}^{\prime}+\mathscr{T}_{i j}^{\prime \prime}+U_{i j}\right) \chi_{j}(\boldsymbol{R}) \\
& =(\underbrace{\sum_{M=1}^{N-1} \frac{-\hbar^{2}}{2 \mu_{M}} \nabla_{M}^{2}}_{\mathscr{T}_{N}=\hat{T}_{\mathrm{N}}}+\underbrace{\sum_{M=1}^{N-1} \frac{-\hbar^{2}}{2 \mu_{M}}\left\langle\phi_{i}(\boldsymbol{r} ; \boldsymbol{R}) \mid \nabla_{M}^{2} \phi_{i}(\boldsymbol{r} ; \boldsymbol{R})\right\rangle}_{\mathscr{T}_{i i}^{\prime \prime}} \\
& +\underbrace{\left\langle\phi_{i}(\boldsymbol{r} ; \boldsymbol{R})\left|\hat{H}_{\mathrm{el}}\right| \phi_{i}(\boldsymbol{r} ; \boldsymbol{R})\right\rangle}_{U_{i i}}) \chi_{i}(\boldsymbol{R}) \\
& +\sum_{j \neq i}^{\infty}(\underbrace{\sum_{M=1}^{N-1} \frac{-\hbar^{2}}{2 \mu_{M}}\left\langle\phi_{i}(\boldsymbol{r} ; \boldsymbol{R}) \mid \nabla_{M} \phi_{j}(\boldsymbol{r} ; \boldsymbol{R})\right\rangle \cdot \nabla_{M}}_{\mathscr{T}_{i j}^{\prime}}+\underbrace{\sum_{M=1}^{N-1} \frac{-\hbar^{2}}{2 \mu_{M}}\left\langle\phi_{i}(\boldsymbol{r} ; \boldsymbol{R}) \mid \nabla_{M}^{2} \phi_{j}(\boldsymbol{r} ; \boldsymbol{R})\right\rangle}_{\mathscr{T}_{i j}^{\prime \prime}} \\
& +\underbrace{\left\langle\phi_{i}(\boldsymbol{r} ; \boldsymbol{R})\left|\hat{H}_{\mathrm{el}}\right| \phi_{j}(\boldsymbol{r} ; \boldsymbol{R})\right\rangle}_{U_{i j}}) \chi_{j}(\boldsymbol{R})
\end{aligned}
$$

$\mathscr{T}_{\mathrm{N}}$ represents the kinetic energy of the nuclei. $U_{i i}(\boldsymbol{R})$ is the effective potential energy surface, which describes the nuclear motion in the potential associated with the electronic state $i$. The diagonal elements $\mathscr{T}_{i i}^{\prime \prime}$ arise from $\hat{T}_{\mathrm{N}}$ acting on the electronic wave function. $\mathscr{T}_{i i}^{\prime \prime}$ are non-adiabatic corrections to the potential energy surface. The offdiagonal elements $\mathscr{T}_{i j}^{\prime}, \mathscr{T}_{i j}^{\prime \prime}$, and $U_{i j}$ govern the interaction between different electronic states. In the adiabatic representation $U_{i j}$ vanishes and only the first and second derivative matrix elements $\mathscr{T}_{i j}^{\prime}$ and $\mathscr{T}_{i j}^{\prime \prime}$ are non-zero. These non-adiabatic interactions are neglected in the Born-Oppenheimer approximation. For reasons of simplicity, the diagonal elements $\mathscr{T}_{i i}^{\prime \prime}$ are also neglected because they are in the same order of magnitude as the off-diagonal $\mathscr{T}_{i j}^{\prime \prime}$ terms. By neglecting the terms governing the non-adiabatic interactions, the quantum mechanical system cannot pass from one PES to another 


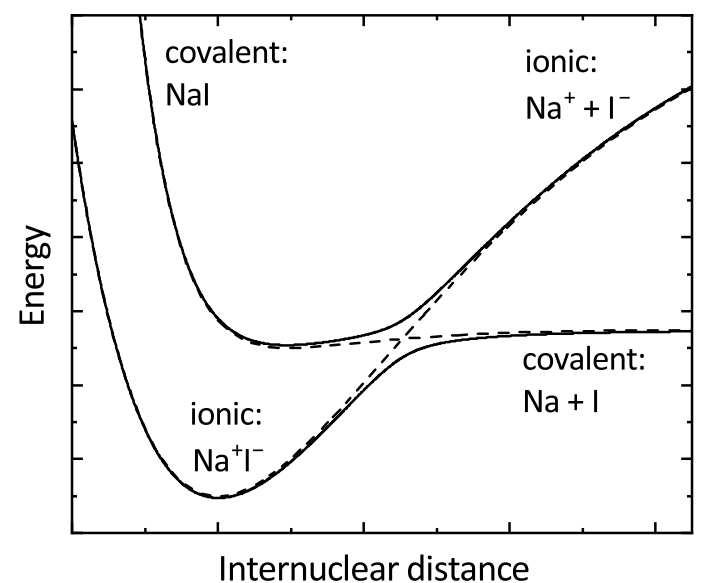

Figure 2.1: Schematic potential diagram of two adiabatic (solid) and diabatic (dashed) potential energy surfaces of sodium iodide. Energies are not true to scale.

one and, thus, evolves electronically adiabatically on just one PES. This is why the Born-Oppenheimer approximation is also referred to as the electronically adiabatic approximation.

The above mathematical digression can be summarized by

$$
\begin{aligned}
\hat{H}\left|\Psi_{i}(\boldsymbol{r}, \boldsymbol{R})\right\rangle & \approx \hat{H}_{\mathrm{BO}}\left|\chi_{i}(\boldsymbol{R})\right\rangle \\
& =\left(\mathscr{T}_{\mathrm{N}}+U_{i i}(\boldsymbol{R})\right)\left|\chi_{i}(\boldsymbol{R})\right\rangle \\
& =\sum_{M=1}^{N-1}-\frac{\hbar^{2}}{2 \mu_{M}} \nabla_{M}^{2}\left|\chi_{i}(\boldsymbol{R})\right\rangle+U_{i i}(\boldsymbol{R}) \chi_{i}(\boldsymbol{R}) \\
& =E_{i}\left|\chi_{i}(\boldsymbol{R})\right\rangle,
\end{aligned}
$$

where $\hat{H}_{\mathrm{BO}}$ is the Born-Oppenheimer-adapted Hamiltonian operator. The first term on the right side of Equation 2.18 describes the kinetic energy of the nuclei while the second term describes the potential energy of nuclei.

Figure 2.1 shows an example of adiabatic potential energy surfaces (solid) representing two electronic states of NaI. In the ground electronic state, the system has an ionic character at short bond lengths and a covalent character at long bond lengths. When ground state NaI dissociates to form a neutral sodium atom and a neutral iodine atom, the electronic configuration switches from ionic to covalent as the nuclear configuration changes such that the system always remains in the ground electronic state. The system evolves adiabatically on the lowest potential energy surface. When, however, ground state $\mathrm{NaI}$ dissociates to $\mathrm{Na}^{+}$and $\mathrm{I}^{-}$, the electronic configuration does not adjust to the 


\section{Theoretical Background}

optimum configuration and stays ionic. The system follows the diabatic curve (dashed) and the dissociation products end up in an electronically excited state.

As indicated in Figure 2.1, adiabatic potential energy curves of the same symmetry do not cross. The Born-Oppenheimer approximation neglects interactions between these curves. However, in regions where adiabatic potential energy surfaces approach one another, off-diagonal matrix elements describing the coupling of states contribute significantly to the total energy of the system and can no longer be neglected. In such regions, the Born-Oppenheimer approximation breaks down and the nuclear momentum may induce a hop between adiabatic potential energy surfaces. These hops are called electronically non-adiabatic transitions. For example, the observation of electronically non-adiabatic dynamics is demonstrated by Rose et al., who excited NaI by generating a wave packet oscillating in the potential energy well of the excited adiabat [41]. Each time the packet passes the region where the diabatic curves cross, a fraction of the molecules undergo non-adiabatic transitions to the lower potential energy surface and dissociate by forming neutral $\mathrm{Na}$ and I atoms. As a consequence, a periodic increase of the Na concentration consistent with the wave package oscillation period is observed.

The non-adiabatic transition probability increases not only near avoided crossing regions where adiabatic potential energy surfaces approach one another, but also when the nuclear velocity along the reaction coordinate increases. This is obvious from $\mathscr{T}_{i j}^{\prime}$ off-diagonal elements, which dominantly govern the non-adiabatic interactions. $\mathscr{T}_{i j}^{\prime}$ is velocity dependent as the differential operator $\nabla_{M}=\partial / \partial R_{M}$ acts on the nuclear wave function $\chi_{i}\left(R_{\mathrm{M}}\right)$. In the two-state model, a measure for the non-adiabatic interaction strength is given by the Massey parameter $\xi$ [42].

$$
\xi=\left|\frac{\hbar \dot{\boldsymbol{R}} \boldsymbol{d}_{i j}}{U_{i}-U_{j}}\right|
$$

$\dot{\boldsymbol{R}}$ is the classical nuclear velocity, $\boldsymbol{d}_{i j}=\left\langle\phi_{i} \mid \nabla_{M} \phi_{j}\right\rangle$ represents non-adiabatic couplings as they appear in $\mathscr{T}_{i j}^{\prime}$, and $U_{i}$ and $U_{j}$ are the adiabatic energies of states $i$ and $j$, respectively. As a rough guide, electronically non-adiabatic transitions are favorable when $\xi \gg 1$, meaning that the nuclear velocity and non-adiabatic couplings are large and the energy difference between the states is small. Following a similar approach, the transition probability $p_{\mathrm{LZ}}$ can be estimated using the Landau-Zener formula [43].

$$
p_{\mathrm{LZ}}=\exp \left(-\xi_{\mathrm{LZ}}\right), \quad \xi_{\mathrm{LZ}}=\frac{\pi(\Delta U)^{2}}{2 \hbar \Delta F \sqrt{2 E_{\mathrm{kin}}(\boldsymbol{R}) / \mu_{M}}}
$$

$E_{\text {kin }}$ is the kinetic energy of nuclei, $\Delta U=U_{i}-U_{j}$, and $\Delta F$ is the difference in the slopes of two asymptotes describing the adiabatic potentials in the avoided crossing 
region. Here, $\Delta F$ is a measure for the non-adiabatic coupling. Further details on non-adiabatic transitions in the two-state approximation are reported elsewhere [44].

\subsubsection{Electronically Non-Adiabatic Dynamics at Surfaces}

As indicated by Equation 2.20, an electronically non-adiabatic event is likely to occur at nuclear configurations where the spacing between adjacent electronic states is small. Due to the lack of a band gap, metal surfaces exhibit a large density of electronic states near the Fermi energy. A system in which a molecule resides in the vicinity of a metal surface has a large number of closely spaced electronic states.

A molecule may accept an electron from the surface forming an anionic species if the energy released by attaching an electron to the molecule exceeds the energy that is needed to elevate a surface electron above the vacuum level. Of course, the electron transfer (ET) can proceed in an electronically adiabatic manner. However, an electronically non-adiabatic electron transfer from the surface to the molecule is likely to occur because of the continuum of occupied electronic states. See Panel (A) of Figure 2.2. Similarly, an anionic molecule that leaves the surface may transfer the electron back into one of the numerous unoccupied electronic states of the metal, as indicated in Panel (B). For systems in which the momentum and kinetic energy coupling terms, $\mathscr{T}_{i j}^{\prime}$ and $\mathscr{T}_{i j}^{\prime \prime}$, respectively, are significantly large, the Born-Oppenheimer approximation breaks down and electronically non-adiabatic dynamics dominate the molecule-surface interaction. This leads researchers to pose questions like, "Can we trust the BornOppenheimer approximation for surface chemistry?" [21] and "How non-adiabatic are surface dynamical processes?" [22] Consequently, electronically non-adiabatic surface dynamics has become an important field of research in surface science, which has been explored by both experimentalists [21-23, 45] and theorists [34, 46-48] for more than 30 years.

A large variety of approaches have been taken to study non-adiabatic surface dynamics. Methods such as reflection absorption infrared spectroscopy (RAIRS), surface enhanced Raman spectroscopy (SERS), and sum frequency generation (SFG) are employed to investigate the vibrational dynamics of adsorbates [49]. The lifetime of vibrationally excited adsorbates is much shorter on metal surfaces than on insulator surfaces because of the non-adiabatic coupling between molecular vibration and electron-hole pairs (EHPs) in the metal $[50,51]$. In surface femtochemistry, the vibrational excitation as well as the desorption and chemical conversion of adsorbates are initiated by photo-excited electron-hole pairs that interact with vibrational degrees of freedom of the adsorbed molecule [52]. Conversely, products of highly exothermic gas-surface reac- 


\section{Theoretical Background}

(A) Incoming molecule

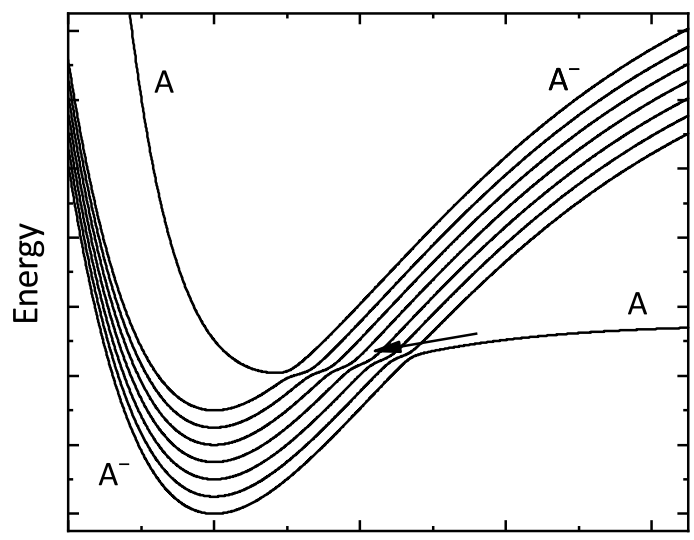

Molecule-surface distance
(B) Scattered anion

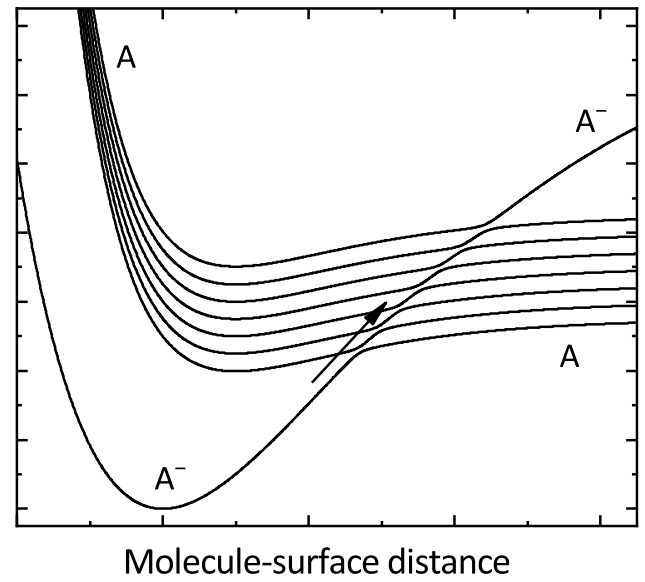

Figure 2.2: Adiabatic potential energy surfaces for a hypothetical molecule $A$ in the vicinity of a metal surface. Panel (A): The approaching molecule may accept an electron from a variety of different electronic states in the surface if electronically non-adiabatic transitions occur. Non-adiabatic transitions are indicated by the arrows. Panel (B): When the anion $\mathrm{A}^{-}$leaves the surface, the electron is transferred back to the surface and an excited electronhole pair is generated if the system suffers non-adiabatic transitions. The figure is adapted from Figure 1 in Reference [21].

tions can relax in an electronically non-adiabatic way by transferring energy to surface electrons, which results in chemiluminescence at or exo-electron emission from surfaces $[53,54]$. The detection of exo-electrons provides direct evidence for electronically nonadiabatic energy dissipation at surfaces. An alternative way to observe non-adiabatic dynamics directly is the measurement of chemicurrents induced by the adsorption or chemical reaction of gaseous molecules at the surface of metal-semiconductor [55] (Schottky diode) or metal-insulator-metal detector devices [56, 57]. With the advent of molecular beams in surface science, quantum-state resolved studies on energy transfer [58, 59] and reaction dynamics [60] in single-bounce molecule-surface collisions has become feasible. The remainder of this section briefly reviews electronically non-adiabatic dynamics in direct molecule-surface scattering events [21, 45, 61], providing background knowledge for experimental results presented and discussed in Chapter 5.

In 1985, Rettner et al. reported the direct vibrational excitation of ground-state NO in collisions with a $\operatorname{Ag}(111)$ surface $[62,63]$. The excitation probability of the $\mathrm{NO}(v=0) \rightarrow \mathrm{NO}(v=1)$ process increases with incidence translational energy and de- 
pends strongly on surface temperature. These findings provide evidence for an electronically non-adiabatic energy transfer in which the nuclear motion of the diatomic molecule couples to excited electron-hole pairs in the surface. An electron transfer mechanism, in which an electron jumps from the surface to the molecule forming a short-lived anionic species, has been proposed in order to explain the experimental observations [64, 65]. See Section 5.3.2 for detailed information about the mechanism. In contrast to nonadiabatic energy transfer [66], electronically adiabatic vibrational excitation at surfaces depends only weakly on the surface temperature. The electronically adiabatic energy transfer - also referred to as mechanical energy transfer - is characterized by a threshold behavior observed in the translational energy dependence of the vibrational excitation probability, which strongly suggests direct conversion of translational to vibrational energy, as reported for the scattering of ammonia at $\mathrm{Au}(111)$ [67].

To date, vibrational excitation via coupling to electron-hole pairs has been reported for a variety of molecule-surface systems, including $\mathrm{NO} / \mathrm{Cu}(110)$ [68], $\mathrm{NO} / \mathrm{Au}(111)$ [6972], $\mathrm{HCl} / \mathrm{Au}(111)$ [73, 74], $\mathrm{CO} / \mathrm{Au}(111)$ [75-77], and $\mathrm{N}_{2} / \mathrm{Pt}(111)$ [78]. The NO/Au(111) system is the most thoroughly studied system and serves as a model system for the determination of absolute vibrational excitation probabilities in single- and multi-quantum vibrational excitation [70]. The Arrhenius-like surface temperature dependence with activation energies similar to the energy spacing between vibrational levels indicates that the excitation energy stems from thermally excited electron-hole pairs [66, 70].

Further insights into non-adiabatic surface dynamics are gained by studies on the $v i$ brational relaxation of excited molecules $(v>0)$ [79-82]. In these studies, the molecule loses vibrational energy during the collision event by exciting electron-hole pairs in the metal. In particular, the scattering of highly vibrationally excited molecules $(v \gg 0)$ attracted special attention because the observed surface dynamics are strongly dominated by electronic non-adiabaticity [25]. $\mathrm{NO}(v=15)$ scattered from $\mathrm{Au}(111)$ undergoes multi-quantum vibrational relaxation, giving rise to a distribution of vibrational energy loss that peaks at $\Delta v=-7,-8$. The vibrational energy loss is inhibited when high- $v$ NO is scattered from $\operatorname{LiF}(001)$ [83], supporting the hypothesis that vibrational energy is gained or lost through non-adiabatic coupling to surface electrons. Further studies investigating the dependence of molecular orientation on the scattering dynamics of highly vibrationally excited NO are consistent with the non-adiabatic picture [84-87].

Perhaps the most compelling evidence for electronic excitation induced by the nuclear motion of a vibrating molecule is the vibrationally promoted emission of electrons observed when highly vibrationally excited NO is scattered from a low-work function surface [88-91]. As the vibrational energy of $\operatorname{NO}(v=15)$ exceeds the work function of a cesium covered $\mathrm{Au}(111)$ surface, multi-quantum vibrational relaxation excites a 


\section{Theoretical Background}

surface electron above the vacuum level. Electron emission is observed as soon as the incident molecule is prepared in a vibrational state that carries along sufficient energy to overcome the work function. Kinetic energy distributions of the ejected electrons are consistent with the vibrational energy loss [92, 93].

In order to elucidate the nature and the underlying mechanism of electronically nonadiabatic dynamics in molecule-surface scattering, it is worth extending the aforementioned experimental findings with $a b$ initio theoretical investigations. The $\mathrm{NO} / \mathrm{Au}(111)$ system serves as a benchmark system for theory because the system exhibits strong non-adiabaticity and has been extensively studied by experimentalists. Two conceptually different approaches have been developed by theorists to explain and predict the scattering outcome. For systems with weak non-adiabatic couplings, electronic friction based models $[26,27,94]$ are commonly used to go beyond the Born-Oppenheimer approximation [95-97]. The coupling of low-energy electron-hole pair excitations to the nuclear motion of molecules at surfaces are treated by introducing a friction force to the classical equations of motion. For systems with strong non-adiabatic interactions, independent-electron surface hopping (IESH) based approaches are used, which explicitly include transitions between adiabatic electronic states [28, 29]. Combined theoretical and experimental studies [30,34,71, 72, 82] show that, for the scattering of highly vibrationally excited $\mathrm{NO}$ from $\mathrm{Au}(111)$, neither the vibrational energy loss nor the incidence translational energy dependence of the relaxation probability is predicted correctly [30]. However, the trend in the velocity dependence is accurately described by a semi-empirical approach assuming anion-mediated vibrational relaxation [98].

\subsection{Electronic Spectroscopy of Carbon Monoxide}

The quantum-state resolved surface scattering of $\mathrm{CO}$ requires extensive knowledge of the energetic structure and spectroscopy of CO. For example, optical transitions observed in resonance-enhanced multi-photon ionization (REMPI) spectra need to be correctly assigned in order to determine final vibrational state and final rotational state distributions. Both quantum-state selective detection and preparation of $\mathrm{CO}$, as mentioned in Section 3.4.1, involve multiple electronic states. The present section provides relevant background about the electronic spectroscopy of CO by briefly introducing the quantum numbers of diatomic molecules, term symbols of diatomic molecules, and selection rules for electronic transitions. For a more detailed description, the reader is referred to Reference [99], which forms the basis of the present section. 
Quantum Numbers of Diatomic Molecules The negatively charged electrons hold the positively charged nuclei of a molecule together. The motion of the electrons determines the electronic state of the molecule. In other words, the electronic state is defined by the orbitals which are occupied by the electrons. The energy difference between electronic states is usually in the range of several $\mathrm{eV}$. In a single atom, electrons move in a spherically symmetric field force. Their motion is described by the orbital angular momentum $\boldsymbol{L}$, as long as the spin of the electrons is neglected. In diatomic molecules, the symmetry is reduced and the specification of $\boldsymbol{L}$ is impossible. Due to the axial symmetry, however, the electronic motion can be described by the projection of $\boldsymbol{L}$ on the internuclear axis, $M_{L}=L, L-1, \ldots,-L$. Note that bold letters indicate a vector while non-bold letters indicate absolute values of quantum numbers. In the absence of a magnetic field, $M_{L}$ states with opposite sign are degenerate. Thus, the component of the electronic orbital angular momentum along the internuclear axis, $\Lambda=\left|M_{L}\right|=$ $0,1,2,3, \ldots, L$, is used to specify the electronic state of a diatomic molecule. States with $\Lambda=0,1,2,3, \ldots$ are designated as $\Sigma, \Pi, \Delta, \Phi, \ldots$ states. $\Pi, \Delta, \Phi, \ldots$ states are doublydegenerate, while $\Sigma$ states are non-degenerate.

Some of these electronic states may exhibit a multiplet structure caused by the interaction with the spin of the electrons. The total spin angular momentum $S$ is either an integer or half integer depending on whether the number of unpaired electrons in the molecule is even or odd, respectively. For $\Sigma$ states, the orientation of the spin vector is fixed in space as long as the molecule is not rotating. In contrast to $\Sigma$ states, $\Pi, \Delta, \Phi, \ldots$ states with $\Lambda \neq 0$ exhibit an internal magnetic field due to the orbital motion of the electrons. This field induces a precession of the spin vector about the internuclear axis. The projection of the spin vector onto the internuclear axis is denoted by the quantum number $\Sigma$, which must not be confused with the aforementioned term symbol $\Sigma$. The spin component along the internuclear axis $\Sigma$ runs in integer steps from $-S$ to $S$. Accordingly, the spin multiplicity is $2 S+1$. Note that the spin quantum number $\Sigma$ is not defined for $\Sigma$ states with $\Lambda=0$.

The multiplet structure of electronic states results from the coupling between $\boldsymbol{\Lambda}$ and $\boldsymbol{\Sigma}$. The projection of the total electronic angular momentum onto the molecular axis is given by $\Omega=|\Lambda+\Sigma|$. The number of different $\Omega$ states is given by the spin multiplicity $2 S+1$.

A certain electronic state of a diatomic molecule is characterized by the term symbol ${ }^{2 S+1} \Lambda_{\Omega}$. The term symbol may also contain information about the symmetry properties of the electronic eigenfunction. For heteronuclear diatomic molecules, + and - indicate whether the sign of the wave function changes upon reflection through a plane that contains the internuclear axis. For homonuclear diatomic molecules, $g$ and $u$ indicate 


\section{Theoretical Background}

the symmetry with respect to molecule-frame inversion on the wave function. In order to label electronic states, term symbols are preceded by an alphabetic letter. Usually, small letters are used when the spin multiplicity differs from that of the ground state. For example, the ground electronic state - commonly denoted by $\mathrm{X}$ - of $\mathrm{CO}$ is $\mathrm{X}^{1} \Sigma^{+}$. The first electronically excited state is $\mathrm{a}^{3} \Pi$, which exhibits a multiplet structure according to ${ }^{3} \Pi_{0},{ }^{3} \Pi_{1}$, and ${ }^{3} \Pi_{2}$.

Rotational Structure of Electronic States So far, the effect of nuclear rotation on the spin and orbital angular momenta of the electrons has not been considered. In the following, the coupling between nuclear rotation and electronic angular momentum will be discussed for electronic states that are relevant to spectroscopic methods applied in this thesis, namely ${ }^{1} \Sigma^{+},{ }^{1} \Pi,{ }^{3} \Sigma^{-}$, and ${ }^{3} \Pi$.

For ${ }^{1} \Sigma$ states, both $S$ and $\Lambda$ are zero and, consequently, the total angular momentum $\boldsymbol{J}$ is simply given by the momentum of nuclear rotation $\boldsymbol{N}$. Rotational energies of CO $\mathrm{X}^{1} \Sigma^{+}$are approximately given by

$$
E_{\mathrm{rot}}=B_{v} J(J+1) .
$$

The rotational constant $B_{v}$ takes the effect of nuclear vibration into account and can be expanded as

$$
B_{v}=B_{e}-\alpha_{e}\left(v+\frac{1}{2}\right)+\ldots
$$

$B_{e}$ is the rotational constant $B_{e}=h /\left(8 \pi^{2} c I_{e}\right)$ with $I_{e}$ being the moment of inertia, $\alpha_{e}$ describes the rotation-vibration interaction, and $v$ is the vibrational quantum number. In general, energy levels of a rotating vibrator can be expressed in terms of the Dunham expansion $[100,101]$.

$$
E(v, J)=\sum_{k, l} Y_{k l}\left(v+\frac{1}{2}\right)^{k}\left[J(J+1)-\Lambda^{2}\right]^{l}
$$

Expansion coefficients $Y_{k l}$ represent vibrational and rotational constants. Dunham coefficients for $\mathrm{CO} \mathrm{X}^{1} \Sigma^{+}$are available from spectroscopic investigations [102]. The rotational structure of ${ }^{1} \Sigma^{+}$states is sketched in Figure 2.3.

Dunham coefficients are also available for $\mathrm{CO} \mathrm{A}^{1} \Pi$ [103]. However, the situation is more complicated for ${ }^{1} \Pi$ states, where $S=0$ and $\Lambda=1$. The two-fold degeneracy of $\Lambda \neq 0$ states is lifted because the nuclear rotation $\boldsymbol{N}$ couples to the orbital angular momentum $\boldsymbol{\Lambda}$ of the electrons. The effect is called $\Lambda$-type doubling. Usually, the splitting is very small and can be neglected for low $J$ states. Since the $\Lambda$-splitting increases with increasing nuclear rotation, the effect may become more relevant for high $J$ states, as indicated in Figure 2.3. 


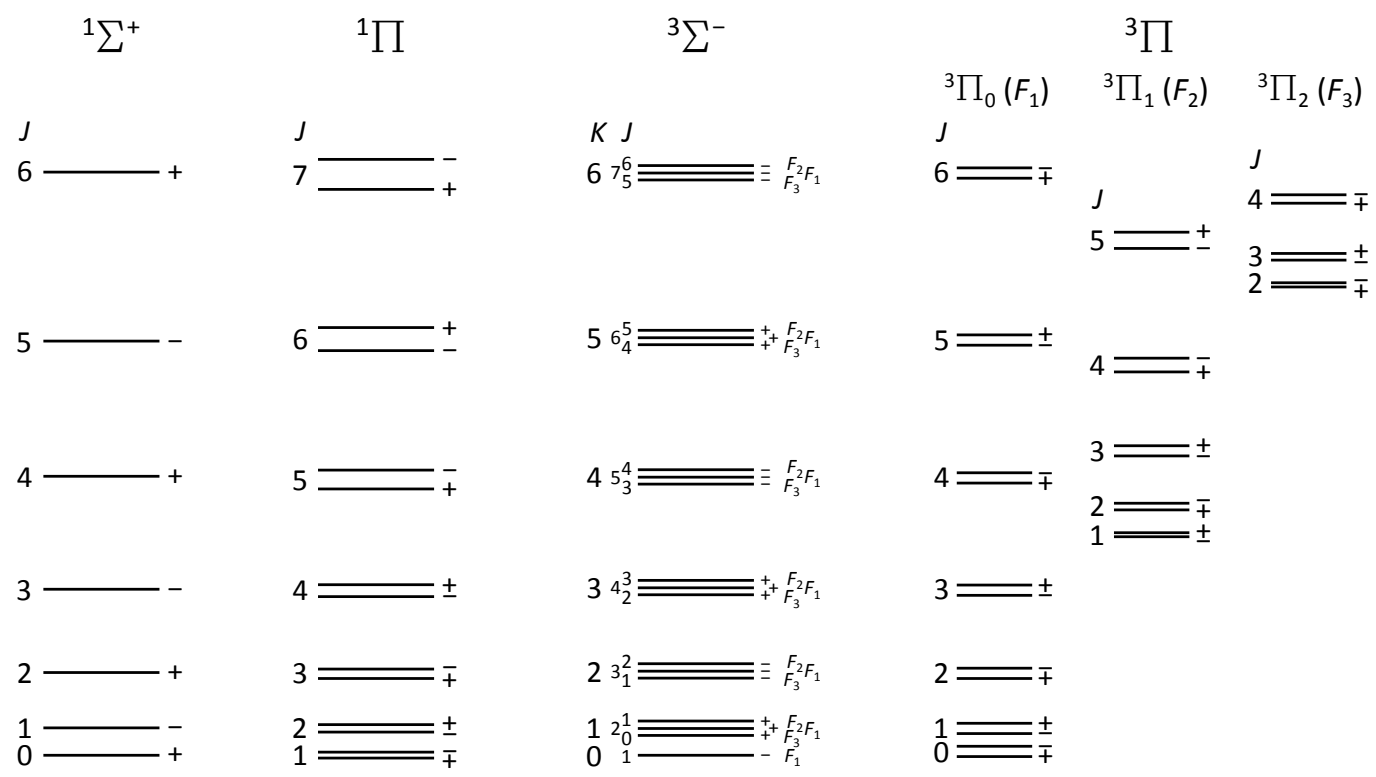

Figure 2.3: Energy level diagram illustrating the rotational structure of the ${ }^{1} \Sigma^{+},{ }^{1} \Pi$, ${ }^{3} \Sigma^{-}$, and ${ }^{3} \Pi$ electronic states of diatomic molecules. Energy spacings between rotational levels, $\Lambda$ doublets, or spin triplets are not drawn to scale.

For ${ }^{3} \Sigma^{-}$states with $S=1$ and $\Lambda=0$, the spin is not coupled to the internuclear axis and the spin quantum number $\Sigma$ is not defined. In this case, the coupling between nuclear rotation and electronic angular momentum is described by Hund's coupling case (b) [104], in which the nuclear rotation $\boldsymbol{N}$ couples to the orbital angular momentum $\boldsymbol{\Lambda}$, if not zero, forming a total angular momentum apart from spin $K=\Lambda, \Lambda+1, \Lambda+2$, etc. $K$ is identical to $N$ when $\Lambda=0 . K$ and $\boldsymbol{S}$ form the total angular momentum $\boldsymbol{J}$ with $J=K+S, K+S-1, \ldots,|K-S|$. For ${ }^{3} \Sigma^{-}$states, the coupling between $\boldsymbol{K}$ and $\boldsymbol{S}$ results in a spin tripling of rotational states. See Figure 2.3.

The rotational structure of ${ }^{3} \Pi$ states with $\Lambda=1$ and $S=1$ can be described by Hund's coupling case (a) [104]. Both the orbital angular momentum and the spin angular momentum couple to the internuclear axis forming the total electronic angular momentum $\Omega$. Here, possible values of $\Omega$ are 0,1 , and 2 , representing three electronic states of the same triplet manifold. See Figure 2.3. $\Omega$ couples to the nuclear rotation $\boldsymbol{N}$ to form the total angular momentum $\boldsymbol{J}$ with $J=\Omega, \Omega+1, \Omega+2$, etc. As in ${ }^{1} \Pi$ states, coupling between the rotation of nuclei and the orbital angular momentum of electrons results in $\Lambda$-type doubling. In contrast to $\Omega=1$ and $2, \Omega=0$ exhibits a relatively 


\section{Theoretical Background}

strong $\Lambda$-splitting for small $J$ and the splitting is almost independent of $J$.

Selection Rules Selection rules tell whether an electric dipole transition between two quantum states is allowed, but they do not predict the transition strength. Generally, selection rules are derived by evaluating the transition dipole moment between two states.

In electric dipole transitions, the selection rule for the total angular momentum is

$$
\Delta J=0, \pm 1, \quad \text { with the restriction } \quad J=0 \nrightarrow J=0 .
$$

Furthermore, the following symmetry selection rules hold for rovibronic transitions.

$$
+\leftrightarrow-\quad+\leftrightarrow+\quad-\leftrightarrow-
$$

For Hund's case (a) and (b) states, the projection of the orbital angular momentum can change by

$$
\Delta \Lambda=0, \pm 1
$$

meaning that $\Sigma-\Sigma, \Sigma-\Pi, \Pi-\Pi, \Pi-\Delta$, etc. transitions are allowed, but transitions such as $\Sigma-\Delta$ are not allowed. In addition, transitions between $\Sigma^{+}$and $\Sigma^{-}$states are not allowed. However, $\Sigma^{+}-\Sigma^{+}$and $\Sigma^{-}-\Sigma^{-}$as well as $\Pi-\Sigma^{+}$and $\Pi-\Sigma^{-}$transitions are allowed. Note that + and - given in the term symbols refer to the parity of the electronic part of the wave function and that the electronic selection rules should not be confused with the rovibronic selection rules given in Equation 2.26.

Experiments presented in the present thesis employ the $\mathrm{A}^{1} \Pi \leftarrow \mathrm{X}^{1} \Sigma^{+}$transition for quantum-state selective detection of $\mathrm{CO}$ molecules. Rotational bands exhibit a P-branch with $\Delta J=-1$, a Q-branch with $\Delta J=0$, and an R-branch with $\Delta J=$ +1 . Note from Figure 2.3 that the lowest $J$ in ${ }^{1} \Pi$ states is $J=1$. Thus, the $\mathrm{P}(1)$ and the $\mathrm{Q}(0)$ transitions do not occur in the spectrum. When calculating rotational transition intensities, the magnetic quantum number $M_{J}$ need to be considered. $\boldsymbol{M}_{\boldsymbol{J}}$ is the projection of $\boldsymbol{J}$ in laboratory frame. The number of possible $M_{J}$ states is $2 J+1$. In general, the selection rule $\Delta M_{J}=0, \pm 1$ holds. However, $M_{J}=0 \rightarrow M_{J}=0$ transitions are forbidden for the Q-branch because of the conservation of angular momentum during the molecule-photon interaction. See Section 4.4 for more details on rotational transition strengths.

Concerning the spin quantum number $S$, the spin multiplicity must not change during electronic transitions.

$$
\Delta S=0
$$


However, this selection may be lifted if the coupling between $\boldsymbol{S}$ and $\boldsymbol{\Lambda}$ is strong, which is the case for heavy nuclei, or if states with different spin multiplicity mix with one another. As mentioned in Section 3.4.1, the laser preparation of highly vibrationally excited CO involves the optical excitation of the $\mathrm{a}^{3} \Pi_{1} \leftarrow \mathrm{X}^{1} \Sigma^{+}$transition and the $\mathrm{e}^{3} \Sigma^{-} \rightarrow \mathrm{X}^{1} \Sigma^{+}$transition. The transition strength of the spin-forbidden $\mathrm{a}^{3} \Pi-\mathrm{X}^{1} \Sigma^{+}$ Cameron band arises from spin-orbit mixing between the $\mathrm{a}^{3} \Pi$ state and the $\mathrm{A}^{1} \Pi$ state [105]. The transition strength of the $\mathrm{e}^{3} \Sigma^{-} \rightarrow \mathrm{X}^{1} \Sigma^{+}$transition originates from a perturbation that affects only low $J$ states of $\mathrm{e}^{3} \Sigma^{-}(v=12)$. Due to the mixing of $\mathrm{e}^{3} \Sigma^{-}(v=12)$ with $\mathrm{A}^{1} \Pi(v=8)$, the $\mathrm{e}^{3} \Sigma^{-}(v=12, J=1)$ triplet state attains partial singlet character. In addition, the $\Sigma^{-} \leftrightarrow \Sigma^{+}$selection rule is weakened because of the $\Pi$ character. As a result, transitions from $\mathrm{e}^{3} \Sigma^{-}(v=12, J=1)$ back into the $\mathrm{X}^{1} \Sigma^{+}$manifold are possible. See Reference [106] for further details on the $\mathrm{e}^{3} \Sigma^{-}(v=12) \sim \mathrm{A}^{1} \Pi(v=8)$ perturbation.

Vibronic Transitions $\mathrm{A}^{1} \Pi\left(v^{\prime}\right) \leftarrow \mathrm{X}^{1} \Sigma^{+}\left(v^{\prime \prime}\right)$ REMPI spectra presented in Section 5.3 show a number of different vibrational bands $v^{\prime}-v^{\prime \prime}$. Each vibrational band consists of rotational lines that obey the aforementioned selection rules. Concerning vibrational bands, there is no restriction for $\Delta v$ in electronic transitions. However, the transition probability of different vibrational bands may vary drastically. The relative transition probability of vibrational bands in excitation spectra are given by the so-called FranckCondon factor

$$
\left|\int \psi_{v}^{\prime *} \psi_{v}^{\prime \prime} \mathrm{d} \tau_{v}\right|^{2}
$$

which represents the overlap integral of the vibrational wave function of the lower state, $\psi_{v}^{\prime \prime}$, and that of the upper state, $\psi_{v}^{\prime}$.

When, however, considering spontaneous emission from an electronically excited state, the vibrational population distribution in the lower electronic state is governed by the Einstein $A$ coefficient, which is proportional to the Franck-Condon factor multiplied with the cubed transition frequency $\nu$.

$$
A \propto\left|\int \psi_{v}^{* *} \psi_{v}^{\prime \prime} \mathrm{d} \tau_{v}\right|^{2} \nu^{3}
$$

\subsection{Stark Deceleration of Molecular Beams}

This section provides a brief introduction to the Stark effect and how it can be exploited to manipulate the velocity of molecular beams. Special emphasis is put on the Stark effect in CO. For a detailed description of the Stark effect in diatomic molecules, the reader is referred to References $[18,99]$. The use of the Stark effect for the manipulation of molecular beams is reviewed elsewhere [18, 107, 108]. 


\section{Theoretical Background}

The Stark effect describes the force that acts on an electric dipole in the presence of an electric field. While an electric charge is translationally accelerated in the presence of a homogeneous electric field, an electric dipole merely feels an orienting force. In an inhomogeneous electric field, however, the electric dipole experiences translational acceleration. Depending on its orientation with respect to the electric field lines, the dipole is attracted to or repelled from areas with increasing field strength. A dipole that is attracted is called high-field seeking and a dipole that is repelled is called low-field seeking.

The Stark effect can be observed for molecules that possess a permanent electric dipole moment and for atoms in which an electric dipole moment is induced by the external field. In the presence of an electric field, certain quantum states may shift in energy. High-field seeking states are stabilized in energy while low-field seeking states are destabilized. This results in a shift or splitting of spectral lines, firstly observed in 1913 by Johannes Stark who investigated the influence of electric fields on the spectral properties of hydrogen and helium atoms [109].

In order to understand the Stark effect in diatomic molecules, a closer look at the energetic structure of quantum states is required. As introduced in Section 2.2, the total angular momentum $\boldsymbol{J}$ includes the orbital motion of electrons, the spin of electrons, and the nuclear rotation. Each $J$ state is $2 J+1$ degenerate. The interaction between the molecule and the field induces a precession of $\boldsymbol{J}$ about an axis that is parallel to the direction of the electric field lines. The component of the total angular momentum along the field direction (space quantization) is then given by $M_{J}=-J,-J+1, \ldots, J$. Thus, the presence of an electric field lifts the degeneracy of $J$ states. Since the sense of nuclear rotation in the electric field has no effect on the energy of the system, $M_{J}$ states with opposite sign are equal in energy. Accordingly, the electric field induces a splitting into $J+1$ sets of states which are doubly degenerate except for the non-degenerate $M_{J}=0$ state.

In the ground electronic state, $\mathrm{X}^{1} \Sigma^{+}, \mathrm{CO}$ has a small permanent dipole moment of $0.11 \mathrm{D}$ [110]. However, $\Sigma$ states do not show a linear Stark splitting because the electric dipole moment of the molecule is oriented perpendicular to the total angular momentum. Consequently, the mean dipole moment along the electric field direction is zero. However, due to the interaction with the field, $\Sigma$ states may have a second order Stark effect, which is generally much weaker than the first order Stark effect. The splitting increases with the square of the electric field strength.

In its first electronically excited state, $\mathrm{a}^{3} \Pi, \mathrm{CO}$ has a much higher dipole moment which is $1.37 \mathrm{D}$ [111] and exhibits a first order Stark effect. The lifetime of the metastable $\mathrm{a}^{3} \Pi_{1}(v=0, J=1)$ state is $2.63 \mathrm{~ms}$ [112] which makes it suitable for molecular beam 
(A)

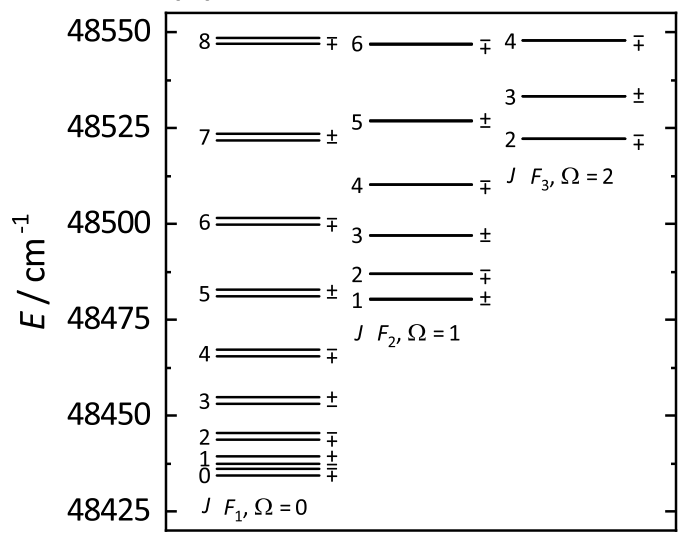

(B)

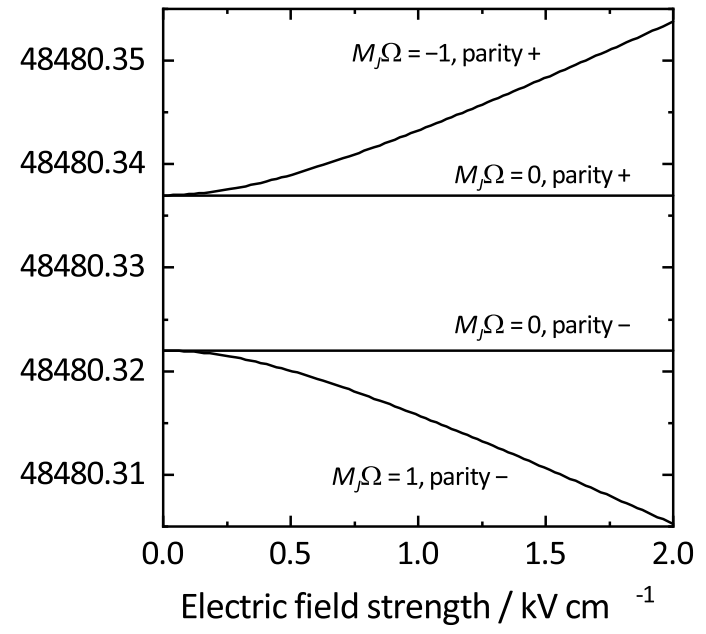

Figure 2.4: Panel (A) illustrates the electronic fine structure of $\mathrm{CO}^{3} \Pi(v=0)$. Panel (B) depicts the + and - parity levels of $\mathrm{CO}^{3} \Pi_{1}(v=0, J=1)$. The first order Stark splitting of the $\Lambda$-doublet is shown as a function of electric field strength.

experiments. The electronic fine structure of $\mathrm{CO} \mathrm{a}^{3} \Pi$ is illustrated in Panel (A) of Figure 2.4. Energy levels are calculated [113] by using effective Hamiltonians [114, 115] for vibronic excitation, nuclear rotation, spin-orbit coupling, spin-rotation coupling, and spin-spin coupling [116]. The relevant molecular constants are given in Reference [111]. Panel (B) illustrates the Stark effect of $\mathrm{CO} \mathrm{a}^{3} \Pi_{1}(v=0, J=1)$ at low electric field strength. In the presence of an electric field, the + parity level splits into two components characterized by $M_{J} \Omega=0$ and $M_{J} \Omega=-1$. While $M_{J} \Omega=0$ is not affected by the field, $M_{J} \Omega=-1$ rises in energy with increasing field strength. Describing the electronic wave functions of $\mathrm{CO} \mathrm{a}^{3} \Pi$ with a Hund's case (a) basis set $\left|J, M_{J}, \Omega\right\rangle$, the $M_{J} \Omega=-1$ component comprises of the $|1,1,-1\rangle$ state and the $|1,-1,1\rangle$ state. Since the energy of these states increases with electric field strength, the mean dipole moment of the rotating molecule is oriented antiparallel with respect to the electric field vector, which makes these states low-field seeking states. The - parity level of the $\mathrm{a}^{3} \Pi_{1}(v=0, J=1) \Lambda$-doublet splits into a $M_{J} \Omega=0$ component, which is also unaffected by the electric field, and a $M_{J} \Omega=1$ component, which comprises of the high-field seeking $|1,1,1\rangle$ and $|1,-1,-1\rangle$ states.

The first order Stark energy is defined as $W_{\mathrm{St}}=-\vec{\mu} \cdot \vec{E}$, where $\vec{\mu}$ is the electric dipole moment vector and $\vec{E}$ is the electric field vector. For a Hund's coupling case 


\section{Theoretical Background}

(a) molecule, the first order Stark energy shift of $\Lambda$-doublet levels with opposite sign is given by

$$
\left\langle J, M_{J}, \Omega, \pm|-\vec{\mu} \vec{E}| J, M_{J}, \Omega, \mp\right\rangle=-\frac{M_{J} \Omega}{J(J+1)}|\vec{\mu}||\vec{E}| .
$$

$|\vec{\mu}|$ is the absolute value of the electric dipole moment, and $|\vec{E}|$ is the absolute value of the electric field strength. $J, M_{J}$, and $\Omega$ are quantum numbers specifying the rotational state, as introduced in Section 2.2. For small electric field strength, the energy of the mixing $\Lambda$-doublet states is approximately given by the effective $2 \times 2$ Hamiltonian matrix

$$
\left(\begin{array}{cc}
\frac{E_{\Lambda}}{2} & -\frac{M_{J} \Omega}{J(J+1)}|\vec{\mu}||\vec{E}| \\
-\frac{M_{J} \Omega}{J(J+1)}|\vec{\mu}||\vec{E}| & -\frac{E_{\Lambda}}{2}
\end{array}\right)
$$

where $E_{\Lambda}$ is the zero-field $\Lambda$ splitting. The eigenvalues of this matrix are given by

$$
W_{\mathrm{St}}= \pm \sqrt{\left(\frac{E_{\Lambda}}{2}\right)^{2}+\left(-\frac{M_{J} \Omega}{J(J+1)}|\vec{\mu}||\vec{E}|\right)^{2}} .
$$

Panel (B) of Figure 2.4 shows a plot of these eigenenergies.

The Stark effect can be exploited in many different ways to manipulate molecular beams, for example in order to orient molecules in the laboratory frame, to select certain quantum states, to change the propagation direction of molecular beams, or to alter their speed. In the present thesis, experiments are described in which a Stark decelerator is used for precise tuning of the velocity of a CO molecular beam. Prior to Stark deceleration, the low-field seeking component of laser-prepared $\mathrm{CO}^{3} \Pi_{1}(v=0, J=1,+)$ is selected by a hexapole device, which focuses the metastable beam into the decelerator.

The hexapole device comprises of six parallel oriented stainless steel rods which are positioned equidistantly on a circle. The main molecular beam axis runs through the center of this circle. The rods are set under high voltage with alternating polarity, creating an inhomogeneous electric field perpendicular to the propagation direction of the molecular beam. If the $\mathrm{CO} \mathrm{a}^{3} \Pi_{1}(v=0, J=1,+)$ beam entering the hexapole is slightly tilted with respect to the hexapole axis, the low-field seeking $M_{J} \Omega=-1$ component is bent and focused onto the hexapole axis where the electric field strength is weakest [117]. Thus, the hexapole serves as a state-selecting device [118-120].

Changing the speed of molecular beams requires the use of time-varying inhomogeneous electric fields that vary along the propagation direction of the molecular beam. The principle of Stark deceleration is sketched in Figure 2.5. A heteronuclear diatomic molecule prepared in a low-field seeking quantum state approaches the inhomogeneous electric field generated between a pair of rod-shaped high voltage electrodes. When the molecule enters the electric field, it is slowed down until it arrives at a point where the 

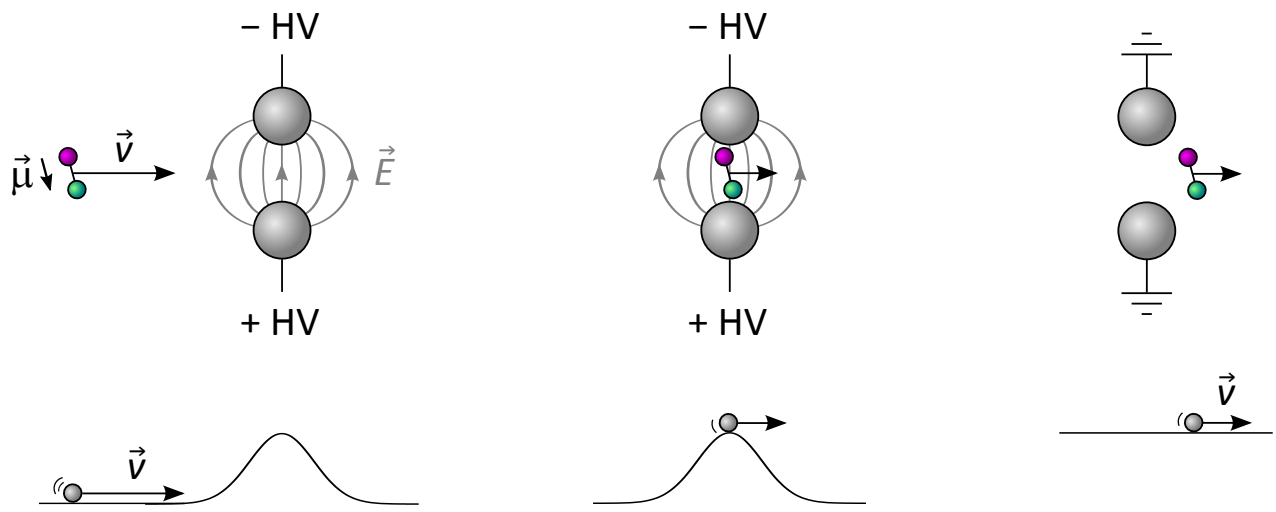

Time

Figure 2.5: Stark deceleration of a low-field seeking diatomic molecule in a timevarying electric field $\vec{E}$. Electric dipole moment and velocity of the molecule are indicated by $\vec{\mu}$ and $\vec{v}$, respectively. The electric field is generated between two rod-shaped high voltage (HV) electrodes. The translational energy loss is illustrated in a simplified picture which shows a ball rolling up a potential energy hill.

electrode-electrode distance is shortest and the electric field is strongest. The molecule will regain its translational energy when it exits the electric field. If, however, the electric field is switched off when the molecule is located directly in between the electrodes, it will keep its reduced velocity. By repeating the process of deceleration with a Stark decelerator consistent of a large number of electrode pairs that can be switched between high voltage and ground at appropriate timings, a molecular beam can be slowed down significantly $[18,108]$. Since low-field seeking molecules avoid getting close to the electrodes, the molecular beam is bunched on the main molecular beam axis and, thus, exhibits low divergence as it traverses through the assembly of high-voltage electrodes. It should also be mentioned that the decelerator is also capable of accelerating a molecular beam if the high voltage is switched on when the molecules are about to leave the electrode pair. When the device is operated in this mode, a low-field seeking molecule moves from an area with high electric field strength to an area with low electric field strength and, thus, gets accelerated.

Although the working principle of a Stark decelerator was developed in the late 1950s $[121,122]$ and some attempts have been made in the 1960s to build a Stark decelerator 


\section{Theoretical Background}

[123, 124], the first molecular Stark decelerator was not successfully operated until 1999 [17]. Bethlem et al. used a $35 \mathrm{~cm}$ long Stark decelerator consistent of 63 electric field stages to slow down a CO molecular beam from $225 \mathrm{~m} / \mathrm{s}$ to $98 \mathrm{~m} / \mathrm{s}$. Typically, the $\mathrm{a}^{3} \Pi_{1}(v=0, J=1,+)$ low-field seeking state is used for the Stark deceleration of CO molecular beams $[17,125,126]$. The Stark decelerator described in the present thesis is made up of 131 synchronously pulsed electric field stages. See Section 3.2 for further details. Each pair of electrodes can create an electric field strength of $80 \mathrm{kV} / \mathrm{cm}$, which results in a translational energy loss of up to $1 \mathrm{~cm}^{-1}$ per deceleration stage. Details on the construction of the decelerator and its physical properties are documented elsewhere $[126,127]$. 


\section{Experimental}

Many of the experiments presented in this thesis, in particular those that involve stateto-state surface scattering of highly vibrationally excited CO, are performed using a conventional molecular beam-surface scattering apparatus. See Section 3.1. Experiments that involve the scattering of Stark-decelerated beams of $\mathrm{CO}$ are performed in a newly developed apparatus combining a Stark decelerator-based molecular beam source with an ultra-high vacuum (UHV) beam-surface scattering chamber. See Section 3.2. The present chapter introduces the reader to experimental setups, laser systems, and experimental procedures used throughout the course of this thesis.

\subsection{Beam-Surface Scattering Apparatus}

Thy dynamics of highly vibrationally excited $\mathrm{CO}$ at gold and silver surfaces is investigated using an experimental setup that was formerly used by Bartels et al. to study the effect of molecular orientation on electron-transfer reactions at surfaces [84, 86, 128]. By removing the orientation electrode and by implementing a new home-built nozzle and a new multi-channel plate (MCP) detector, Krüger et al. improved significantly the detection efficiency of surface scattered molecules, paving the way for state-to-state scattering of highly vibrationally excited CO. In the following, the experimental setup in its present state is described in detail. A description of the apparatus can also be found elsewhere [129]. Figure 3.1 shows a schematic drawing of the setup.

A home-built Even-Lavie type [130] nozzle, designed by Prof. Dr. Schwarzer, is used as the molecular beam source. The nozzle produces supersonic jets at a $10 \mathrm{~Hz}$ repetition rate. Molecular beams of $\mathrm{CO}$ are generated by co-expanding a gas mixture of $\mathrm{CO}$ and $\mathrm{H}_{2}$ through a $500 \mu \mathrm{m}$ wide hole. The translational energy of the beam is tuned by varying the mixing ratio of the gases. The approximate velocity, $v$, can be estimated from the average mass of molecules in the mixture, $\bar{m}$, the average heat capacity at constant pressure, $\bar{c}_{p}$, and the temperature of the nozzle, $T_{0}$, which is kept at room temperature [131].

$$
v=\sqrt{\frac{2}{\bar{m}} \bar{c}_{p} T_{0}}
$$




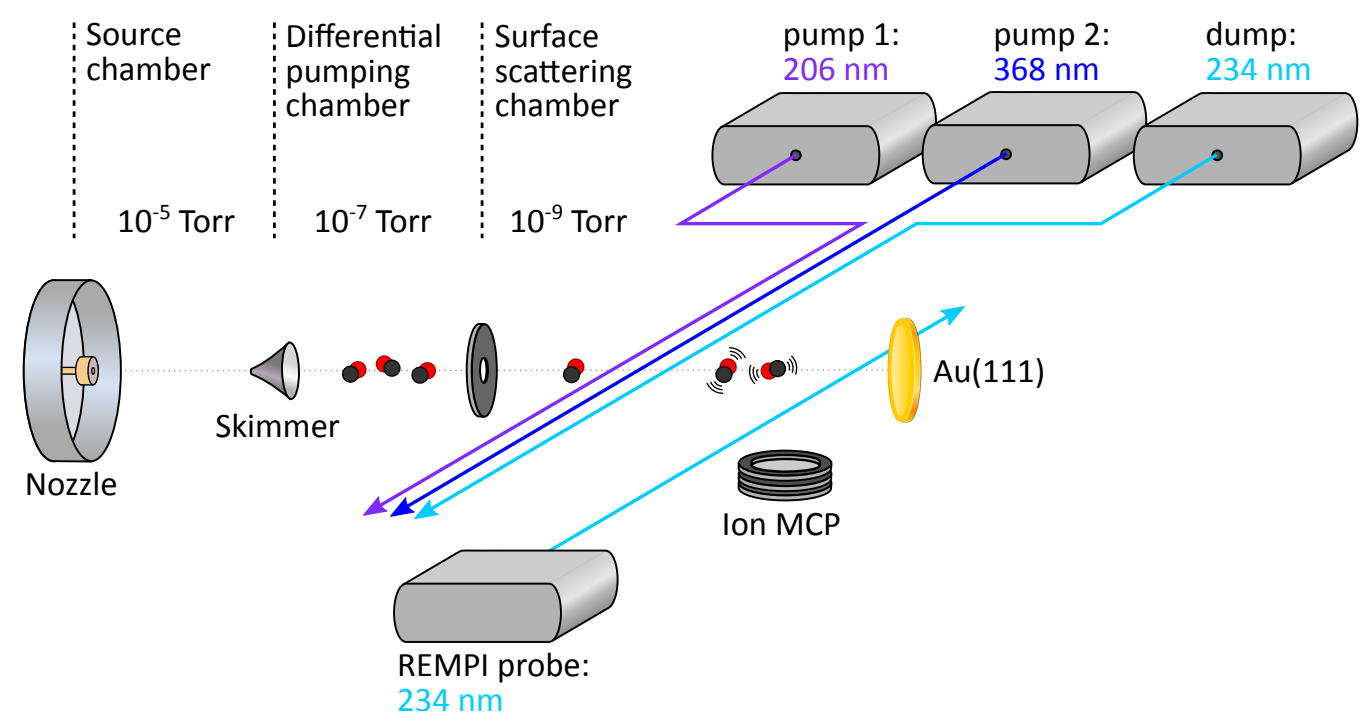

Figure 3.1: Schematic drawing of the molecular beam-surface scattering apparatus. The setup consists of three differentially pumped chambers. Supersonic molecular beams are generated by co-expanding $\mathrm{CO}$ seeded in $\mathrm{H}_{2}$. After passing a skimmer and an aperture, the beam arrives in the UHV surface scattering chamber where high $v$ states are prepared by laser excitation. Surface scattered CO is REMPI ionized and detected by the MCP detector. Pressures are given for a running molecular beam.

Final translational energies are determined more precisely via time-of-flight experiments. See Section 4.1. For instance, the expansion of gas mixtures composed of 25\%, 10\%, and $8 \% \mathrm{CO}$ seeded in $\mathrm{H}_{2}$ yields translational energies of $0.26 \mathrm{eV}, 0.41 \mathrm{eV}$, and $0.57 \mathrm{eV}$, respectively. The stagnation pressure in front of the nozzle is 7 bar. With the nozzle turned off, the source chamber is maintained at $10^{-9}$ Torr. Turning on the nozzle causes the pressure to rise to $10^{-6}-10^{-5}$ Torr.

The molecular beam enters a differentially pumped chamber by passing through a $2 \mathrm{~mm}$ electro-formed skimmer (Ni Model 2, Beam Dynamics, Inc.). The differentially pumped chamber is connected to the surface scattering chamber by a $2 \mathrm{~mm}$ aperture, through which the beam arrives in the scattering chamber. The pressure in the scattering chamber is kept at $10^{-10}$ Torr. The pressure rises to $10^{-9}$ Torr when the molecular beam is turned on.

The molecular beam is scattered from gold and silver single crystals cut along the (111) face (MaTecK, 99.999\% purity). The crystals are mounted between two tungsten wires which can be resistively heated in order to heat the sample. Cooling is also pos- 
(A) $\mathrm{Au}(111)$

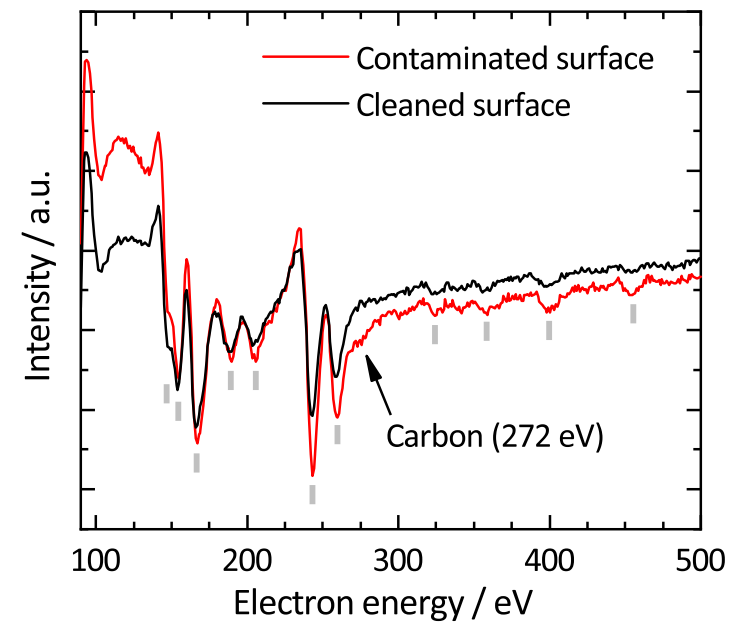

(B) $\mathrm{Ag}(111)$

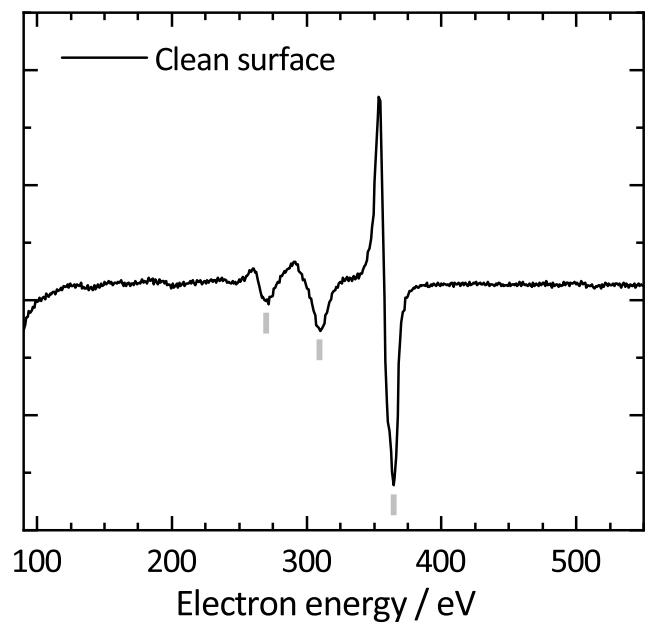

Figure 3.2: Panel (A) shows Auger electron spectra of clean (black) and contaminated (red) $\mathrm{Au}(111)$. The shoulder at $272 \mathrm{eV}$ indicates the presence of carbon at the surface. Peaks at 148, 154, 166, 188, 203, 243, 254, 323, 357, 398, and $453 \mathrm{eV}$ (gray markers) can be attributed to Au. Panel (B) shows the Auger electron spectrum of sputtered and annealed $\operatorname{Ag}(111)$. Peaks at 271,310 , and $364 \mathrm{eV}$ (gray markers) can be attributed to Ag. Spurious contamination of carbon (expected at $272 \mathrm{eV}$ ) and oxygen (expected at 468, 483, and $503 \mathrm{eV}$ ) cannot be found. Auger scans up to $2400 \mathrm{eV}$ (not shown) also prove the absence of sulfur (expected at 2043, $2117 \mathrm{eV}$ ) [132].

sible by cooling down the sample mount with liquid nitrogen. The surface temperature is probed by a K-type thermocouple. The sample mount is connected to a high precision manipulator (U.H.V. Instruments, Burlington, Ontario, Canada, model number: 30007651110 ), capable of $250 \mathrm{~mm}$ translation along the vertical $z$-axis, $25 \mathrm{~mm}$ along the $x$ - and $y$-axis, and $360^{\circ}$ rotation around the $z$-axis.

Standard cleaning procedures under UHV conditions are used in order to prepare atomically clean and flat surfaces [133]. The surface is sputtered with $\mathrm{Ar}^{+}$-ions for $20 \mathrm{~min}$ (LK Technologies, NGI3000-SE, $3 \mathrm{kV}$ beam energy, $20 \mathrm{~mA}$ emission current, $12 \mu \mathrm{A}$ surface current, $1.5 \times 10^{-6}$ Torr). Subsequent annealing ( $970 \mathrm{~K}$ for gold, $870 \mathrm{~K}$ for silver) for $20 \mathrm{~min}$ completes the cleaning process. Afterwards, the surface is kept in UHV, where it stays clean over several hours. Even after $9 \mathrm{~h}$ of exposure to the molecular beam, no spurious contaminations can be found on the surface. The chemical cleanliness of the surface is probed by Auger electron spectroscopy (ESA 150, Staib 


\section{Experimental}

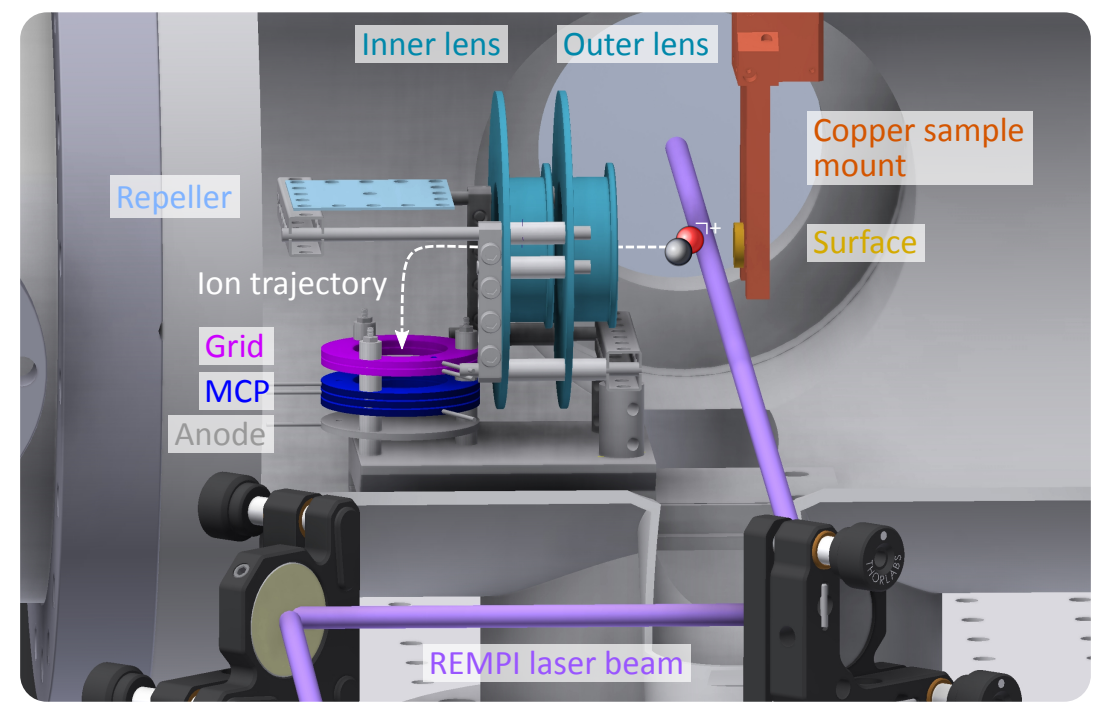

Figure 3.3: Side view of the surface scattering chamber interior, showing surface (yellow) and multi-channel plate (MCP) detector. The detector is comprised of ion lenses (turquois), ion repeller (cyan), grid (magenta), two multichannel plates (blue), and anode (gray). Incident or scattered CO is ionized by the REMPI laser (purple) and guided to the MCP plates.

Instruments, $4 \mathrm{kV}$ beam energy, $1.5 \mathrm{~A}$ emission current, $1350 \mathrm{~V}$ detector voltage). See Figure 3.2. The structure of the surface has been probed many times in the past by low energy electron diffraction (LEED), proving that sputtering followed by annealing creates flat surfaces with a negligible step density [134]. The surface scattering chamber is equipped with a residual gas analyzer (SRS, RGA 200), which can be used to probe the composition of residual gas in the UHV chamber. A leak valve (VACGEN Ltd., LVM series, ZLVM940R) is mounted on the UHV chamber allowing foreign gases to be let into the chamber in a controlled manner. The same leak valve is used for argon ion sputtering.

Two vacuum windows (fused silica, DN $100 \mathrm{CF}$ ) provide laser access to the surface scattering chamber. Laser beams traverse the vacuum chamber perpendicular to the molecular beam. The large windows allow laser excitation of incident molecules $22 \mathrm{~mm}$ in front of the surface and laser detection of scattered molecules as close to the surface as possible.

Quantum-state specific detection of surface scattered molecules is achieved by resonance-enhanced multi-photon ionization (REMPI) spectroscopy. The absorption of two photons ionizes $\mathrm{CO}$ and creates $\mathrm{CO}^{+}$. A set of electrodes (ion lenses and repeller) 
guide the positively charged molecules to the multi-channel plate (MCP, tectra, chevron configuration, $25 \mathrm{~mm}$ diameter) detector. See Figure 3.3. The voltage on the MCP plates is increased after the REMPI laser is fired in order to prevent saturation of the detector caused by UV stray light. Inner lens, outer lens, repeller, grid and MCP are usually operated at $-200,-900,+100,-2000$, and $+1400-1900 \mathrm{~V}$, respectively, in order to detect positively charged ions. Note that laser ionized CO is initially accelerated parallel to the molecular beam axis, such that the detector is capable of differentiating between incident molecules, which are moving away from the outer lens, and scattered molecules, which are moving towards the outer lens, by time-of-flight.

In order to keep the pressure in the surface scattering chamber as low as possible while the molecular beam is turned on, the whole machine is composed of three differentially pumped vacuum chambers, as sketched in Figure 3.1. The surface scattering chamber is pumped by a turbomolecular pump (Osaka Vacuum Ltd., TNP062, 420 l/s), which is backed by a smaller turbomolecular pump (Pfeiffer Vacuum, TPU 170, 170l/s). UHV is generated by baking the surface scattering chamber at $373 \mathrm{~K}$ for two days. The source chamber (Osaka Vacuum Ltd., TS443, 400 l/s) and the differential chamber (Leybold, Turbovac 360CSV, 360l/s), which is located between the source and the surface scattering chamber, are evacuated by turbomolecular pumps as well. All turbomolecular pumps are backed by rotary vane pumps (Trivac, W42 and W47). The gas station, which is used to prepare gas mixtures for molecular beams, can be evacuated by a rotary vane pump as well (Edwards, E2M30).

\subsection{The Stark Machine}

In addition to the beam-surface scattering apparatus described in Section 3.1, experiments were also performed using the so-called Stark machine. The Stark machine is a newly developed experimental setup $[113,127,135]$ that combines the technique of Stark deceleration $[17,18,107,136]$ for precise translational energy control of molecular beams with a UHV beam-surface scattering chamber. A detailed description of the apparatus has been published in Reference [126].

A pulsed supersonic molecular beam is produced in the source chamber by expanding a gas mixture consistent of $20 \%$ CO seeded in Xe. A molecular beam with a mean velocity of $360 \mathrm{~m} / \mathrm{s}$ is produced by cooling the nozzle to $260 \mathrm{~K}$ (home-built Even-Lavie type nozzle [130] designed by Prof. Dr. Schwarzer, with a $500 \mu \mathrm{m}$ diameter aperture). Nozzle cooling is achieved by exposing the nozzle housing to cool dry $\mathrm{N}_{2}$ gas, which is pre-cooled by flowing through a copper coil submerged in liquid nitrogen. Note that the nozzle is tilted by $3.5^{\circ}$ with respect to the main molecular beam axis of the machine. See 


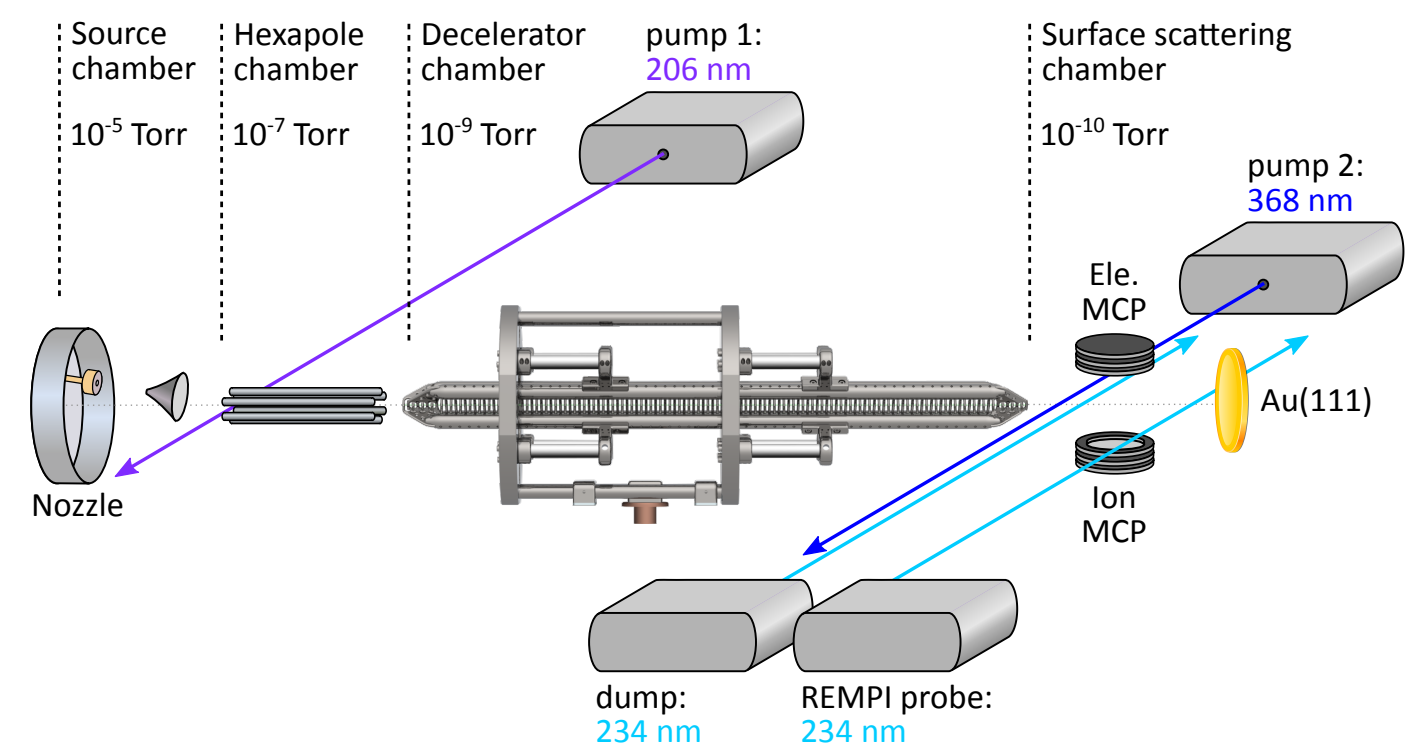

Figure 3.4: Schematic drawing of the Stark machine. A supersonic molecular beam is produced by expanding $20 \% \mathrm{CO}$ seeded in Xe through a pulsed nozzle. The first pump laser prepares $\mathrm{CO}$ in the metastable $\mathrm{a}^{3} \Pi$ state. Having passed through the hexapole and the Stark decelerator, the metastable beam arrives in the UHV surface scattering chamber, which houses the $\mathrm{Au}(111)$ surface, an electron MCP detector, and an ion MCP detector. Laser pulses shown in the drawing refer to the $\mathrm{P}^{3} \mathrm{D}$ pumping scheme described in Section 3.4.1.

Figure 3.4. The source chamber is separated from the hexapole chamber by a flange that serves as a support for a skimmer (Beam Dynamics, Inc., Model 2, Nickel) of $1.5 \mathrm{~mm}$ diameter. The skimmer is located $30 \mathrm{~mm}$ downstream from the nozzle and allows the central part of the supersonic beam to arrive in the hexapole chamber.

The hexapole chamber is equipped with laser access windows mounted at Brewster's angle. Before the molecular beam enters the hexapole device, metastable $\mathrm{CO}$ is generated by exciting the $\mathrm{a}^{3} \Pi_{1}(v=0, J=1,+) \leftarrow \mathrm{X}^{1} \Sigma^{+}(v=0, J=1,-)$ transition with a narrow-bandwidth laser at $206.293 \mathrm{~nm}$. The preparation of $\mathrm{CO} \mathrm{a}^{3} \Pi_{1}$ is necessary in order to exploit the Stark effect for molecular beam manipulation. See Section 2.3. In contrast to $\mathrm{CO}$ in the ground electronic state, metastable $\mathrm{CO}$ exhibits a first order Stark effect and has a sufficiently large dipole moment. The hexapole is used to bend the trajectories of metastable molecules. This separates $\mathrm{CO}^{3} \Pi_{1}(v=0, J=1,+)$ from residual ground state $\mathrm{CO}$ and $\mathrm{Xe}$ carrier gas, both of which are unaffected by the strong 

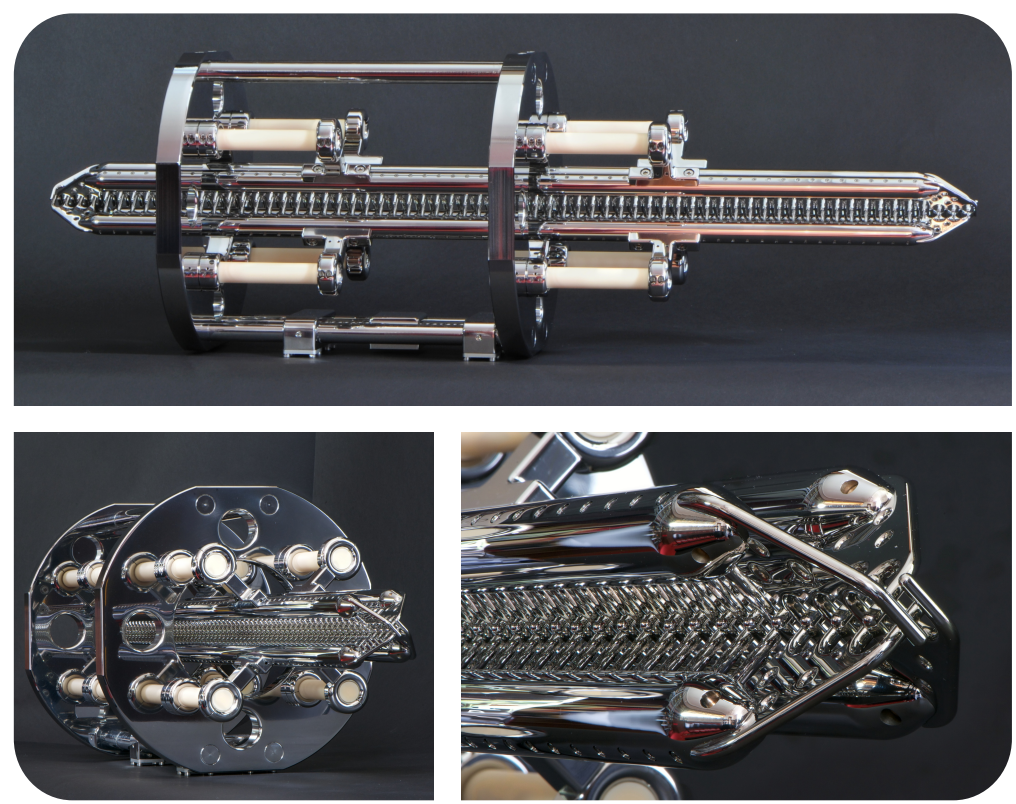

Figure 3.5: Photographs of the Stark decelerator. Photographs by Georg Heyne, FritzHaber-Institute of the Max-Planck-Society, Berlin.

electric fields of the hexapole. Furthermore, the electric fields of the hexapole act as a positive lens that precisely couples the metastable beam into the decelerator device. The hexapole consists of $110 \mathrm{~mm}$ long highly polished stainless steel rods that are operated at $\pm 14 \mathrm{kV}$ (Spellman SL20P1200/SL20N1200 power supply, $20 \mathrm{kV}, 1200 \mathrm{~W}$ ). In order to prevent discharges, high voltage of the hexapole is turned on $5 \mu \mathrm{s}$ after the excitation laser has fired using fast high voltage switches (Behlke Power Electronics GmbH, HTS-201-06-GSM).

The metastable beam enters the decelerator chamber through a $2 \mathrm{~mm}$ orifice. The arrival of metastable $\mathrm{CO}$ in the decelerator chamber is monitored by a solar blind photo multiplier tube (Hamamatsu Photonics, R821, $3.6 \times 10^{5}$ gain, $160 \mathrm{~nm}-320 \mathrm{~nm}$ ) which collects UV photons originating from the radiative decay of $\mathrm{CO} \mathrm{a}^{3} \Pi$. The decelerator chamber houses the $720.5 \mathrm{~mm}$ long Stark decelerator, which consists of 132 pairs of highly polished stainless steel electrodes with hemispherical end caps, forming an array of 131 equidistant electric field stages [127]. Each pair of electrodes consists of two mutually parallel rod-shaped electrodes that are spatially separated by $2 \mathrm{~mm}$. Each rod is $3 \mathrm{~mm}$ thick. The surface-to-surface distance to an adjacent pair of electrodes is $2.5 \mathrm{~mm}$. Each pair of electrodes is oriented perpendicular to the molecular beam axis. Adjacent pairs are rotated $90^{\circ}$ relative to one another. See Figure 3.5. 


\section{Experimental}

All electrodes are mounted to four stainless steel rods, which are held in place by four rod-shaped ceramic insulators. The ceramic insulators connect the high voltage electrodes to the grounded stainless steel support frame. The whole decelerator setup is designed such that the alignment of electrodes survives a bakeout at $100^{\circ} \mathrm{C}$ for several hours. This allows the decelerator to be operated under UHV conditions, as is required for application of the technique to beam-surface scattering experiments.

The decelerator is operated between $\pm 14.0 \mathrm{kV}$ and $\pm 15.5 \mathrm{kV}$ using high voltage power supplies (Spellman SL20P1200/SL20N1200, 20kV, 1200 W). As the molecular beam travels through the decelerator, fast switching between ground and high voltage is achieved by using fast high voltage switches with a rise time of $200 \mathrm{~ns}$ (Behlke Power Electronics GmbH, HTS-201-06-GSM). A burst of triggers is needed to turn on and off the high voltage at correct timings as the molecular beam traverses the 132 pairs of electrodes. The respective trigger timings are listed in the so-called burst file. The burst file is read by the computer program KouDA which feeds the triggers to a delay generator that is directly connected to the high voltage switches. Burst file timings are calculated using the libcoldmol software [137].

In order to generate a molecular beam with a certain final velocity, the burst file with the correct timings must be loaded. The procedure of loading a burst file prior to each experiment, however, complicates experiments in which the velocity needs to be scanned over a wide range in small steps. In the course of the present thesis, a method has been developed that allows automated velocity scans, making velocity scans a convenient procedure. See Section 6.2.1.

The initial velocity of molecules entering the decelerator is $360 \mathrm{~m} / \mathrm{s}$, as determined by the supersonic jet expansion of the cooled $\mathrm{CO} / \mathrm{Xe}$ gas mixture. The Stark decelerator can be used to guide $\left(\phi_{0}=0^{\circ}\right)$, decelerate $\left(\phi_{0}>0^{\circ}\right)$, or accelerate $\left(\phi_{0}<0^{\circ}\right)$ the molecular beam (the phase angle $\phi_{0}$ specifies the molecule's reduced position with respect to the potential of the electric field of one electrode pair). The maximum velocity change accompanied with reasonable beam intensities behind the decelerator is obtained for phase angles of $\phi_{0}=69^{\circ}$ and $\phi_{0}=-90^{\circ}$, corresponding to beam velocities of $99 \pm 7 \mathrm{~m} / \mathrm{s}$ $(1.4 \mathrm{meV})$ and $512 \pm 4 \mathrm{~m} / \mathrm{s}(38 \mathrm{meV})$, respectively. When guiding the beam at a constant velocity of $360 \pm 14 \mathrm{~m} / \mathrm{s}$, the decelerator is operated in $s=3$ mode, meaning that the high voltage is switched every third deceleration stage that is passed by the molecular beam. The $s=3$ mode offers a better longitudinal velocity resolution and provides a more pronounced and homogeneous peak shape (See Reference [127], p. 53 for more information). However, only every third deceleration stage is effectively used in $s=3$ mode. Thus, the decelerator is always operated in $s=1$ mode when decelerating or accelerating the beam, making use of every deceleration stage. 
The end of the Stark decelerator extends into the surface scattering chamber. The end of the decelerator is shielded by a stainless steel cap, which is mounted inside the vacuum chamber. The inside of the cap is highly polished to prevent discharges. The cap is equipped with two fused silica UV windows mounted at Brewster's angle, providing laser access to the last six decelerator stages. The laser beam enters the vacuum chamber through $100 \mathrm{~mm}$ diameter viewports mounted on the surface scattering chamber.

After leaving the decelerator, the metastable beam enters the surface scattering chamber through a $2 \mathrm{~mm}$ diameter circular hole in the cap. The molecular beam is scattered from a $\mathrm{Au}(111)$ (Monocrystals Company, Medina, Ohio, USA, $10 \mathrm{~mm}$ diameter, $2 \mathrm{~mm}$ thickness) surface located $4.5 \mathrm{~cm}$ downstream from the end of the decelerator. The region between decelerator and surface can be accessed by lasers through the aforementioned $100 \mathrm{~mm}$ viewports. Here, lasers are employed to either transfer metastable CO back to the ground electronic state prior to surface scattering or to detect CO molecules by quantum-state specific REMPI spectroscopy. See Figure 3.4.

The arrival of metastable $\mathrm{CO}$ in the surface scattering chamber can be probed in two different manners. First, the de-excitation of $\mathrm{CO} \mathrm{a}^{3} \Pi$ at $\mathrm{Au}(111)$ causes electron emission because the excitation energy of metastable $\mathrm{CO}(6.0 \mathrm{eV})$ exceeds the work function of gold $(5.3 \mathrm{eV})$. Ejected electrons are guided to a multi-channel plate (MCP) detector located above the molecular beam axis (Tectra, MCP 050, chevron configuration, $40 \mathrm{~mm}$ active diameter). Second, metastable CO and laser prepared highly vibrationally excited CO (see Section 3.4.1) can be probed by REMPI spectroscopy. Positively charged ions are guided to a second MCP detector (Hamamatsu, type F1094-01, chevron configuration, $24.8 \mathrm{~mm}$ diameter, $0.48 \mathrm{~mm}$ thickness; formerly used: Tectra, MCP 050, chevron configuration, $40 \mathrm{~mm}$ active diameter) located below the molecular beam axis. See Figure 3.4. Various REMPI schemes for $\mathrm{CO} \mathrm{a}^{3} \Pi$ detection are summarized in Section 3.4.2.

The surface temperature can be varied between $28 \mathrm{~K}$ and the melting point of gold at $1337 \mathrm{~K}$. The gold crystal is mounted between two tungsten wires that can be resistively heated. The wires are connected to the copper sample mount via two sapphire plates. This way, the surface is electrically insulated from the sample mount, but can exchange heat with the sample mount. A so-called coldfinger made from copper connects the sample mount to a water-cooled helium compressor (Advanced Research Systems, $4 \mathrm{HW}$ compressor), which is used to cool the surface. The temperature of the surface is read by an E-type thermocouple. The coldfinger is connected to a manipulator (VG Scienta, Omniax MXZ800, MT211B6S, ZRP100H) with two differentially pumped rotatable flanges (thermionics vacuum products, RNN-100/MS). The system allows rotation about two axes pointing along the $z$ direction, $800 \mathrm{~mm}$ translation along the $z$-axis, and 


\section{Experimental}

$25 \mathrm{~mm}$ translation along the $x$ - and $y$-axis.

The surface scattering chamber is equipped with several surface preparation and analysis tools. A residual gas analyzer (Stanford Research Systems, RGA 200) is used to monitor UHV vacuum conditions and to perform temperature programmed desorption (TPD) measurements. The RGA is shielded by a cylindrical copper cap in order to reduce the background signal in TPD measurements. Molecules desorbing from the surface are allowed to enter the ionization region through a $1 \mathrm{~mm}$ aperture on the front face of the cap. A leak valve connected to a $83 \mathrm{~mm}$ long dosing tube, which has an inner diameter of $500 \mu \mathrm{m}$, is used for controlled deposition of gaseous molecules on the cooled surface.

Prior to any TPD or surface scattering experiment, the $\mathrm{Au}(111)$ surface is cleaned by $\mathrm{Ne}^{+}$sputtering (STAIB Instruments, Ion Source IG-5-C) and subsequent annealing at $900 \mathrm{~K}$, following standard cleaning procedures for well-defined metal surfaces under UHV conditions [133]. $\mathrm{Ne}^{+}$sputtering is used rather than $\mathrm{Ar}^{+}$sputtering in order to minimize background pressure caused by rare gas atoms desorbing from the liquid helium cooled coldfinger. The chemical purity of the surface is probed by Auger electron spectroscopy (STAIB Instruments, Electron Spectrometer ESA 100). See for instance Panel (A) of Figure 3.2.

The work function of the surface can be probed relative to that of a stainless steel plate by using a Kelvin probe (KP Technology, UHVKP020).

The whole experimental setup comprises four vacuum chambers. The source, hexapole, and decelerator chambers are differentially pumped. The decelerator and surface scattering chamber are kept under UHV conditions. The source, decelerator, and surface scattering chamber are pumped by $685 \mathrm{~L} / \mathrm{s}$ turbomolecular pumps (Pfeiffer Vacuum, HiPace 700), while the hexapole chamber is pumped by a $355 \mathrm{~L} / \mathrm{s}$ turbomolecular pump (Pfeiffer Vacuum, HiPace 400). The turbomolecular pump mounted to the surface scattering chamber is backed by a smaller turbomolecular pump (Pfeiffer Vacuum, HiPace 80, 67 L/s). All turbomolecular pumps are backed by three diaphragm pumps (Pfeiffer Vacuum, MVP-070-3, $4.3 \mathrm{~m}^{3} / \mathrm{h}$ ), maintaining a background pressure of $10^{-1}$ Torr. In order to generate UHV in the surface scattering chamber, the machine is baked at $100{ }^{\circ} \mathrm{C}$ for several hours. During the experiments, the surface scattering chamber is maintained at $10^{-10}$ Torr.

There is no discernible pressure rise in the surface scattering chamber when the molecular beam is turned on. This strongly indicates that the number of molecules that enter the surface scattering chamber is extremely small. As a consequence, there was too little signal to allow laser-preparation of ground state CO [106, 138] followed by surface scattering and REMPI detection of scattered molecules. See Section 6.2.2. Thus, 


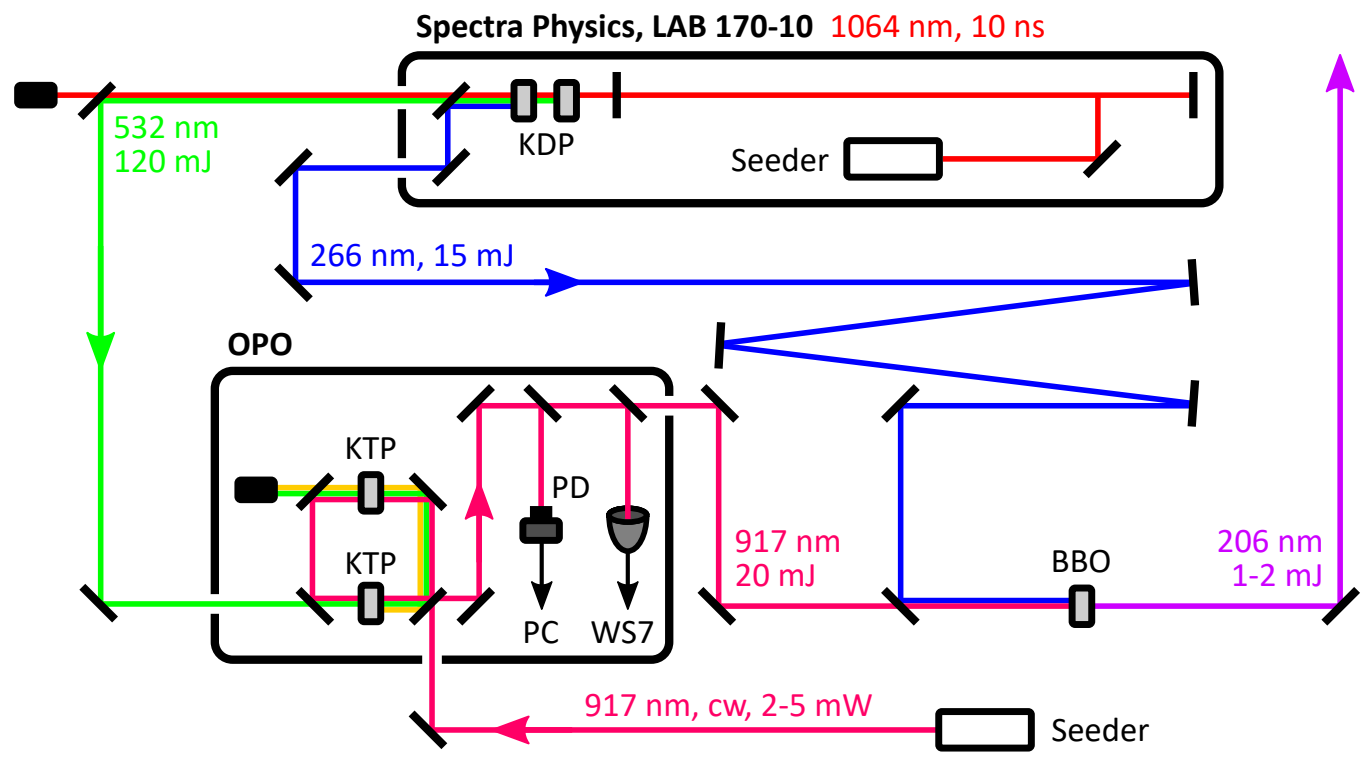

Figure 3.6: The frequency doubled output (green) of a seeded Nd:YAG laser pumps the optical parametric oscillator (OPO). Two potassium titanyl phosphate (KTP) crystals in the OPO's resonator produce a signal beam (pink, $917 \mathrm{~nm}$ ) and an idler beam (yellow) by means of down-conversion. The OPO is seeded by a continuous-wave (cw) diode laser. The OPO output is monitored by a photodiode (PD) and a wavelength meter (WS7). A $\beta$-barium borate (BBO) crystal generates a $206 \mathrm{~nm}$ laser beam (purple) by sum frequency generation (SFG) using the OPO output and the fourth harmonic of the Nd:YAG laser (blue, $266 \mathrm{~nm}$ ).

the Stark decelerator-based molecular beam source was removed from the experimental setup in 2018. The Stark decelerator moved back to Prof. Dr. Gerard Meijer's group at the Fritz-Haber-Institute in Berlin where it was designed and built.

\subsection{Laser Systems}

Narrow Bandwidth Nanosecond Laser Pulses in the Deep UV Narrow bandwidth laser pulses at $206 \mathrm{~nm}$ are produced by a home-built laser setup based on the description published by Velarde et al. [139]. Figure 3.6 shows a sketch of the setup which is briefly explained in the following. A narrow bandwidth nanosecond laser pulse at $206 \mathrm{~nm}$ with 1-2 mJ pulse energy is generated by means of sum frequency generation (SFG) in a 


\section{Experimental}

$\beta$-barium borate $(\mathrm{BBO})$ crystal using the Fourier-transform limited fourth harmonic (266 nm, $15 \mathrm{~mJ})$ of an injection-seeded (Spectra Physics, Injection Seeder Model 6350) Nd:YAG laser (Spectra Physics, pulsed Nd:YAG laser, LAB 170-10) and the output $(917 \mathrm{~nm}, 20 \mathrm{~mJ})$ of a home-built injection-seeded optical parametric oscillator (OPO). The OPO is pumped with $120 \mathrm{~mJ}$ of the second harmonic $(532 \mathrm{~nm})$ produced by the same Nd:YAG laser. The resonator of the OPO is equipped with two nonlinear potassium titanyl phosphate (KTP) crystals. Down conversion of the pump beam yields a socalled signal beam $(917 \mathrm{~nm}, 20 \mathrm{~mJ})$ and an idler beam. The idler beam is discarded. In order to generate Fourier-transform limited nanosecond laser pulses, the OPO is seeded by a continuous-wave (cw) external cavity diode laser (Toptica Photonics, DL Pro 100, $917 \mathrm{~nm}, 2-5 \mathrm{~mW}$ ) operated on a single longitudinal mode. The OPO output is monitored by a photodiode (PD) which measures the intensity of the light leaving the resonator as a probe of resonator setting. The length of the resonator is precisely adjusted by a piezoelectric transducer that serves as a mount for one of the four resonator mirrors. The wavelength of the OPO output is monitored by a wavelength meter (HighFinesse, WS7).

The light source generates 1-2 mJ of $206.293 \mathrm{~nm}$ with a spectral width of $300 \mathrm{MHz}$ and excites the spin-forbidden $\mathrm{a}^{3} \Pi_{1}(v=0, J=1,+) \leftarrow \mathrm{X}^{1} \Sigma^{+}(v=0, J=1,-)$ transition in CO. This transition is the first pump step in the $\mathrm{P}^{3} \mathrm{D}$ pumping scheme [106]. See Section 3.4.1. Even though the transition derives intensity from spin-orbit mixing between $\mathrm{a}^{3} \Pi$ and $\mathrm{A}^{1} \Pi[105]$, the transition is not saturated.

Spectra Physics Dye Laser A pulsed dye laser (Spectra Physics, Quanta Ray PDL-2 Pulsed Dye laser) is used to produce light for the second pump step in two different pumping schemes $[106,138]$. The dye laser is pumped with the second harmonic of a pulsed Nd:YAG laser (Continuum, Powerlite 8000, PL 8010). The dye laser is operated with Rhodamine 6G dissolved in methanol or with Styryl 8 (Exciton, LDS 751) dissolved in ethanol generating $563-566 \mathrm{~nm}$ and $736 \mathrm{~nm}$, respectively, with a spectral linewidth of $0.2 \mathrm{~cm}^{-1}$. If needed, the frequency of the dye laser output is doubled by a nonlinear BBO crystal. The laser setup is used to excite the $\mathrm{d}^{3} \Delta_{1}(v=5) \leftarrow \mathrm{a}^{3} \Pi_{1}(v=0)$ transition at $563 \mathrm{~nm}$, the $\mathrm{b}^{3} \Sigma^{+}(v=0) \leftarrow \mathrm{a}^{3} \Pi_{1}(v=0)$ transition at $283 \mathrm{~nm}$, or the $\mathrm{e}^{3} \Sigma^{-}(v=12) \leftarrow \mathrm{a}^{3} \Pi_{1}(v=0)$ transition at $368 \mathrm{~nm}$.

Sirah Dye Lasers Two pulsed dye lasers (Sirah Laser- und Plasmatechnik GmbH, Cobra Stretch and Precision Scan) are used to excite the dump transition in the $\mathrm{P}^{3} \mathrm{D}$ pumping scheme [106] and to detect $\mathrm{CO} \mathrm{X} \mathrm{X}^{1} \Sigma^{+}$and $\mathrm{CO} \mathrm{a}^{3} \Pi_{1}$ by means of REMPI. The dye lasers are operated with Coumarin C460. Pulsed Nd:YAG lasers (Continuum, 
Powerlite 8000, PL 8010, and Spectra Physics, LAB 230-10) serve as pumping sources. The frequency of the dye laser output is doubled by a nonlinear BBO crystal. The setups are used to drive the dump transition at $234.160 \mathrm{~nm}$ or to utilize various REMPI schemes for the detection of $\mathrm{CO} \mathrm{X}^{1} \Sigma^{+}(v=0), \mathrm{CO} \mathrm{X}^{1} \Sigma^{+}(v \geq 14)$, and $\mathrm{CO} \mathrm{a}^{3} \Pi_{1}(v=0)$ between $228 \mathrm{~nm}$ and $245 \mathrm{~nm}$.

Continuum OPO Laser System The commercially available tunable OPO (Continuum, Sunlite ${ }^{\mathrm{TM}}$ EX OPO FX-1) is capable of producing any wavelength between $225.5 \mathrm{~nm}$ and $1750 \mathrm{~nm}$ with a linewidth smaller than $0.3 \mathrm{~cm}^{-1}$. The system consists of an injection-seeded pulsed Nd:YAG laser pump source, an OPO, and a subsequent FX-1 doubling unit. The setup is used to excite the dump transition in the $\mathrm{P}^{3} \mathrm{D}$ pumping scheme [106] at $234.160 \mathrm{~nm}$.

\subsection{Experimental Procedures}

\subsubsection{Preparation and Detection of $\mathrm{CO} \mathrm{X}^{1} \Sigma^{+}(v=17)$}

Stimulated emission pumping (SEP) is the method of choice for preparing molecular beams consisting of diatomic molecules in high $v$ states [140,141]. As the preparation by one-photon excitation is not feasible, SEP populates highly vibrationally excited states via an excited electronic state. This requires two light sources, namely one pump laser and one dump laser. However, the preparation of highly vibrationally excited $\mathrm{CO}$ is challenging because high lying electronically excited states require vacuum ultraviolet (VUV) radiation for the pump step. Thus, a new optical pumping scheme has been developed recently which omits the use of VUV radiation by employing two UV pump lasers [106]. This so-called pump-pump-perturb-dump $\left(\mathrm{P}^{3} \mathrm{D}\right)$ scheme is used to prepare $\mathrm{CO} \mathrm{X}^{1} \Sigma^{+}(v=17)$.

Figure 3.7 shows electronic and vibrational states that are relevant to the $\mathrm{P}^{3} \mathrm{D}$ optical preparation scheme. The first pump laser excites the $\mathrm{a}^{3} \Pi_{1}(v=0, J=1,+) \leftarrow$ $\mathrm{X}^{1} \Sigma^{+}(v=0, J=1,-)$ transition. Even though this spin-forbidden transition derives intensity from spin-orbit mixing of the $A^{1} \Pi$ state with the $a^{3} \Pi$ state [105], it cannot be saturated with a narrow-bandwidth laser $(\Delta \nu=300 \mathrm{MHz})$. The first pump step is therefore the bottleneck of the $\mathrm{P}^{3} \mathrm{D}$ pumping scheme. The second pump excites the $\mathrm{e}^{3} \Sigma^{-}(v=12, J=1,-) \leftarrow \mathrm{a}^{3} \Pi_{1}(v=0, J=1,+)$ transition. Since the $\mathrm{e}^{3} \Sigma^{-}(v=12, J=1)$ state mixes with the $\mathrm{A}^{1} \Pi_{1}(v=8, J=1)$ state, high $v$ states of the ground electronic state can be accessed from $\mathrm{e}^{3} \Sigma^{-}(v=12, J=1)$ because of large Franck-Condon overlap between the $\mathrm{A}^{1} \Pi(v=8)$ state and high vibrational levels 


\section{Experimental}

of the $\mathrm{X}^{1} \Sigma^{+}$state. The population transfer to high $v$ states can be achieved in two different ways. First, a number of different high $v$ states is populated by radiative decay of the $\mathrm{e}^{3} \Sigma^{-}(v=12, J=1)$ state. The resulting vibrational state population distribution is then determined by the Einstein $A$ coefficients. As the Einstein $A$ coefficient of a certain transition is given by the respective Franck-Condon factor multiplied by the cubed transition frequency, the population of high $v$ states by means of spontaneous emission is often referred to as Franck-Condon pumping (FCP). Second, the population



Figure 3.7: Potential energy diagram illustrating electronic states relevant to the $\mathrm{P}^{3} \mathrm{D}$ optical pumping scheme. Pump 1 excites the nominally spin-forbidden $\mathrm{a}^{3} \Pi_{1}(v=0) \leftarrow \mathrm{X}^{1} \Sigma^{+}(v=0)$ transition. Subsequently, pump 2 excites the $\mathrm{e}^{3} \Sigma^{-}(v=12) \leftarrow \mathrm{a}^{3} \Pi_{1}(v=0)$ transition. The dump laser stimulates the $\mathrm{e}^{3} \Sigma^{-}(v=12) \rightarrow \mathrm{X}^{1} \Sigma^{+}(v=17)$ transition, which derives intensity from the $\mathrm{e}^{3} \Sigma^{-}(v=12, J=1) \sim \mathrm{A}^{1} \Pi(v=8, J=1)$ perturbation. Highly vibrationally excited $\mathrm{CO}$ is probed by $(1+1) \mathrm{A}^{1} \Pi(v=8) \leftarrow \mathrm{X}^{1} \Sigma^{+}(v=17)$ REMPI spectroscopy. The figure is adapted from Fig. 1 in Ref. [106]. 
(A)

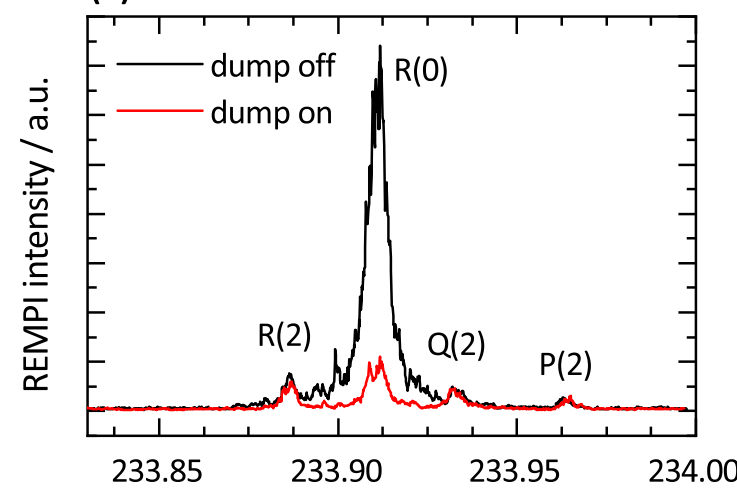

(B)

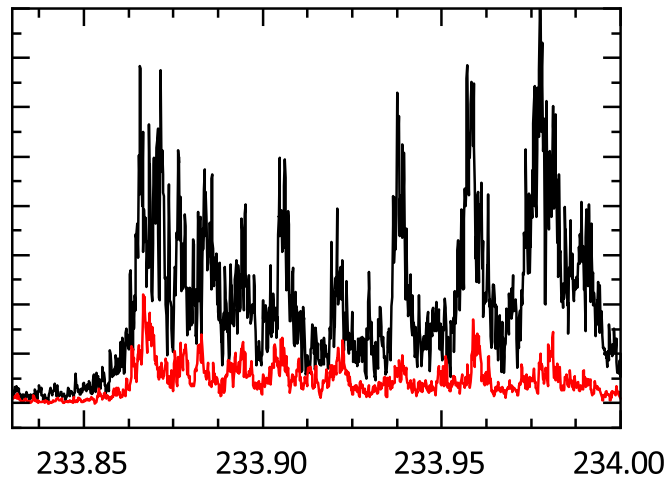

Figure 3.8: Panel (A) and (B) show $(1+1) \mathrm{A}^{1} \Pi\left(v^{\prime}=8\right) \leftarrow \mathrm{X}^{1} \Sigma^{+}\left(v^{\prime \prime}=17\right)$ REMPI spectra of incident and surface scattered $\mathrm{CO}(v=17)$, respectively. Spectra are shown for the dump laser turned on (black) and turned off (red). The red traces indicate the background signal induced by FCP. The spectra of incoming $\mathrm{CO}$ contains four transitions because two rotational states can be accessed by FCP. The spectrum of surface scattered $\mathrm{CO}$ is more complex because the surface collision results in rotational excitation.

in a single rovibrational state can be selectively increased by stimulated emission, which requires the use of a so-called dump laser. The frequency of the dump laser is chosen such that it drives the $\mathrm{e}^{3} \Sigma^{-}(v=12, J=1,-) \rightarrow \mathrm{X}^{1} \Sigma^{+}(v=17, J=0,+)$ transition. Any observation reported in the present thesis concerning the surface scattering of highly vibrationally excited $\mathrm{CO}$ results from the scattering of incident CO prepared in the $\mathrm{X}^{1} \Sigma^{+}(v=17, J=0,+)$ state. The presence of $\mathrm{CO}(v=17)$ in the incoming beam and the presence of different high $v$ states in the scattered beam is probed by $(1+1) \mathrm{A}^{1} \Pi\left(v^{\prime}, J^{\prime}\right) \leftarrow \mathrm{X}^{1} \Sigma^{+}\left(v^{\prime \prime}, J^{\prime \prime}\right)$ REMPI spectroscopy. $\mathrm{X}^{1} \Sigma^{+}(v=17)$ can be probed via $\mathrm{A}^{1} \Pi(v=7)$ or $\mathrm{A}^{1} \Pi(v=8)$. Figure 3.8 shows REMPI spectra of incident and scattered $\mathrm{CO}$, illustrating the effect of the dump laser.

Figure 3.8 demonstrates that stimulated emission does not suppress FCP. The presence of high $v$ states other than $v=17$ in the incoming beam leads to an undesired background signal in the detection of surface scattered molecules. Great care is taken in the analysis to correct for the FCP induced background. This is basically achieved by repeating the surface scattering measurement for the dump laser turned on and off, as indicated in Panel (B) of Figure 3.8. See Section 4.3 for more details.

As the first pump laser produces long-lived metastable $\mathrm{CO}$ with a lifetime of $2.63 \mathrm{~ms}$ 


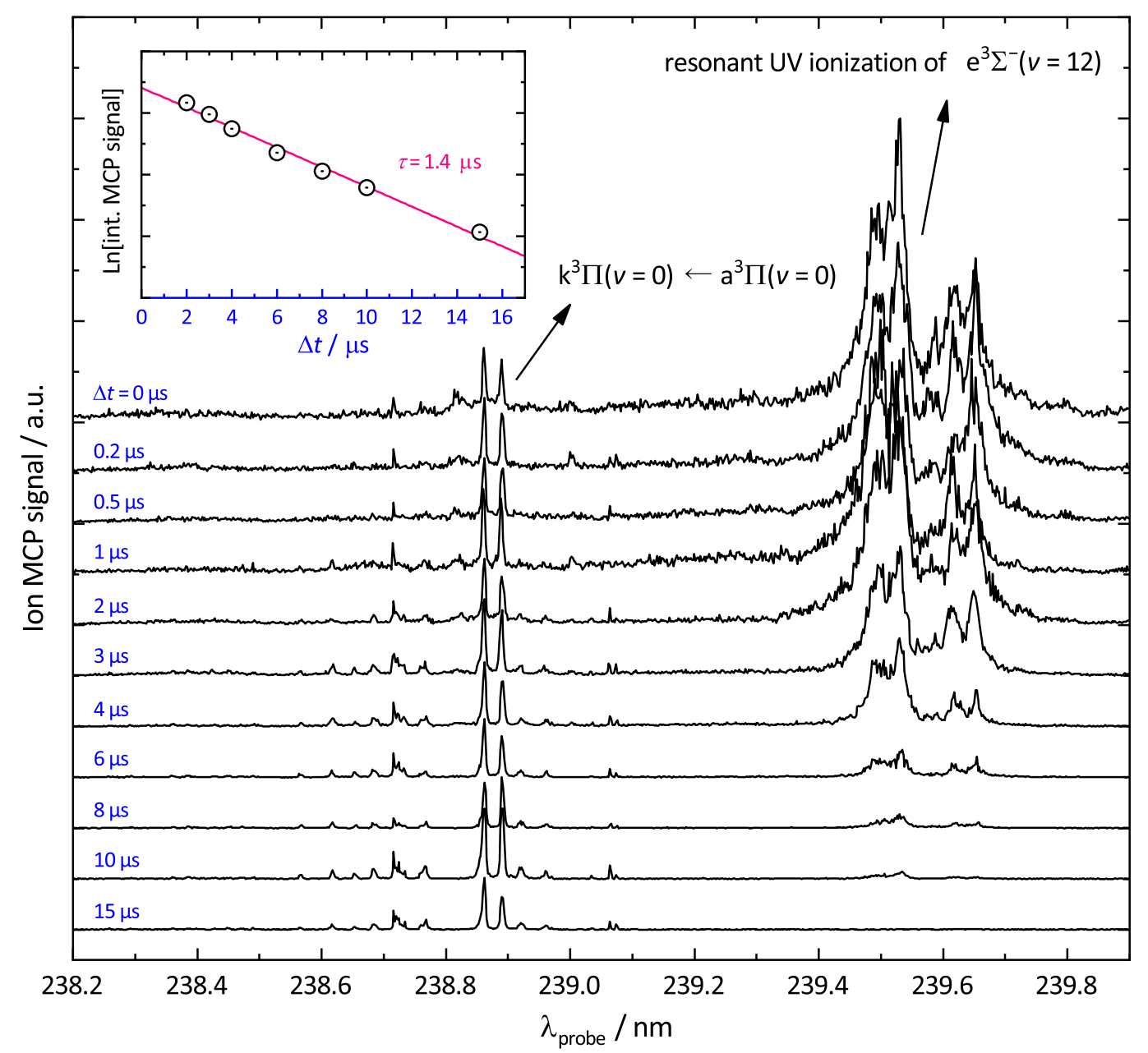

Figure 3.9: Photoionization spectra after the first and second pump lasers have prepared $\mathrm{CO} \mathrm{e}^{3} \Sigma^{-}(v=12, J=1)$. Spectra are recorded for different time delays $\Delta t$ between the second pump laser and the probe laser. The dump laser is not used. The band around $238.9 \mathrm{~nm}$ is attributed to $(1+1) \mathrm{k}^{3} \Pi(v=0) \leftarrow \mathrm{a}^{3} \Pi_{1}(v=0)$ REMPI. Over the whole spectral range $(238.2-239.8 \mathrm{~nm})$, the probe laser ionizes $\mathrm{e}^{3} \Sigma^{-}(v=12, J=1)$, which is seen as a constant ion signal in the $\Delta t=0 \mu$ s trace and which should not be mistaken as noise. Note that the signal decays as the time delay $\Delta t$ is increased. At around $239.5 \mathrm{~nm}$, an extremely broad and intense band arises, which probes the $\mathrm{e}^{3} \Sigma^{-}(v=12)$ state presumably via a short-lived resonance above the $\mathrm{CO}$ ionization potential. The inset shows the natural logarithm of the integrated band $(239.2-239.9 \mathrm{~nm})$ as a function of $\Delta t$. The linear dependence indicates a monoexponential decay, from which the $\mathrm{e}^{3} \Sigma^{-}\left(v=12, J=1,-, F_{3}\right)$ state's lifetime of $\tau=1.4 \pm 0.2 \mu$ s is deduced. 
Table 3.1: Exact wavelengths and recommended laser settings for the pump-pumpperturb-dump [106] preparation of $\mathrm{CO} \mathrm{X}^{1} \Sigma^{+}(v=17)$. Transitions marked with an asterisk are used in this thesis to produce $\operatorname{CO}(v=17, J=0)$.

\begin{tabular}{llllll}
\hline \hline Laser & Wavelength & Polarization & Power & Diameter & Timing \\
\hline Pump 1 & $206.293 \mathrm{~nm}, \mathrm{Q}_{2}(1)^{*}$ & $\mathrm{p} ; \mathrm{s}$ & $1-2 \mathrm{~mJ}$ & $3 \mathrm{~mm}$ & $0 \mathrm{~s}$ \\
\hline Pump 2 & $368.863 \mathrm{~nm}, \mathrm{Q}_{32}{ }^{*}$ & $\mathrm{p} ; \mathrm{s}$ & $2-3 \mathrm{~mJ}$ & $3 \mathrm{~mm}$ & $50 \mathrm{~ns}$ \\
& $368.930 \mathrm{~nm}, \mathrm{Q}_{12}$ & & & & \\
\hline Dump & when $Q_{32}$ is pumped & $\mathrm{p} ; \mathrm{s}$ & $3 \mathrm{~mJ}$ & $3 \mathrm{~mm}$ & $100 \mathrm{~ns}$ \\
& dumping into $J=0:$ & & & & \\
& $234.160 \mathrm{~nm}^{*}$ & & & & \\
& dumping into $J=2:$ & & & \\
& $234.212 \mathrm{~nm}$ & & & & \\
\end{tabular}

when $Q_{12}$ is pumped

dumping into $J=0$ :

$234.194 \mathrm{~nm}$

dumping into $J=2$ :

$234.249 \mathrm{~nm}$

\begin{tabular}{llllll}
\hline Probe & $233.890 \mathrm{~nm}, \mathrm{R}(2)$ & $\mathrm{p} ; \mathrm{s}$ & $1-2 \mathrm{~mJ}$ & $3 \mathrm{~mm}$ & $>10 \mu \mathrm{s}$ \\
& $233.910 \mathrm{~nm}, \mathrm{R}(0)$ & & & \\
& $233.930 \mathrm{~nm}, \mathrm{Q}(2)$ & & & \\
& $233.965 \mathrm{~nm}, \mathrm{P}(2)$ & & & \\
\hline
\end{tabular}

[112], residual $\mathrm{CO} \mathrm{a}^{3} \Pi_{1}(v=0)$ approaches the surface along with $\mathrm{CO} \mathrm{X}^{1} \Sigma^{+}(v=17)$. However, metastable CO does not contribute to the observed background signal because $\mathrm{CO} \mathrm{a}^{3} \Pi_{1}(v=0)$ does not relax to high $v$ states of the ground electronic state during collisions with gold or silver surfaces. This is obvious as the presence of highly vibrationally excited $\mathrm{CO}$ in the scattered beam disappears when the second pump laser is turned off. Furthermore, a contribution to the background signal due to $\mathrm{CO}^{3} \Sigma^{-}(v=12)$ scattering is excluded. $\mathrm{e}^{3} \Sigma^{-}(v=12)$ decays radiatively during the flight time to the surface. Based on resonant UV ionization spectroscopy, the lifetime of $\mathrm{e}^{3} \Sigma^{-}\left(v=12, J=1,-, F_{3}\right)$ is estimated to be $1.4 \pm 0.2 \mu \mathrm{s}$. See Figure 3.9. This value agrees with $1.8 \pm 0.3 \mu$ s obtained from laser-induced fluorescence measurements [106]. The flight time to the surface is at least $10 \mu \mathrm{s}$ as highly vibrationally excited CO is prepared $22 \mathrm{~mm}$ in front of the surface and the beam with the highest velocity travels at $2000 \mathrm{~m} / \mathrm{s}$. 


\section{Experimental}

Table 3.1 summarizes wavelengths and laser settings for the $\mathrm{P}^{3} \mathrm{D}$ optical preparation of $\mathrm{CO} \mathrm{X}^{1} \Sigma^{+}(v=17)$ in molecular beams. Laser systems that are used to generate the required transition frequencies are described in Section 3.3.

\subsubsection{Detection of $\mathrm{CO} \mathrm{a}^{3} \Pi_{1}(v=0)$}

The $\mathrm{a}^{3} \Pi$ state is the lowest-lying electronically excited state of CO. Due to its long lifetime of $2.63 \mathrm{~ms}$ [112] and its large dipole moment of $1.37 \mathrm{D}[111]$, the $\mathrm{a}^{3} \Pi$ state is of great importance in the Stark-manipulation of $\mathrm{CO}$ molecular beams and in optical pumping schemes such as $\mathrm{P}^{3} \mathrm{D}$, where it serves as an intermediate state allowing the excitation to higher states without using VUV light sources. Fortunately, spin-orbit mixing between the $\mathrm{a}^{3} \Pi$ and $\mathrm{A}^{1} \Pi$ state allows optical access from the singlet ground electronic state [105]. In the present thesis, metastable $\mathrm{CO}$ is produced by exciting the $\mathrm{Q}_{2}(1)$ transition, $\mathrm{a}^{3} \Pi_{1}(v=0, J=1,+) \leftarrow \mathrm{X}^{1} \Sigma^{+}(v=0, J=1,-)$, at $206.293 \mathrm{~nm}$. As the detection of $\mathrm{CO} \mathrm{a}^{3} \Pi$ is necessary in order to locate the metastable beam in the beam-scattering apparatus and to align subsequent excitation lasers, this chapter briefly summarizes different ways to detect metastable CO. Figure 3.10 shows various resonance-enhanced multi-photon ionization (REMPI) spectra, including (1+1) REMPI via the $\mathrm{k}^{3} \Pi$ and the $\mathrm{b}^{3} \Sigma^{+}$state, and $\left(1+1^{\prime}\right)$ REMPI via the $\mathrm{e}^{3} \Sigma^{-}$state. Useful wavelengths are given in Table 3.2 .

The positions of $\mathrm{k}^{3} \Pi\left(v^{\prime}=0,1,2,3\right) \leftarrow \mathrm{a}^{3} \Pi_{1}\left(v^{\prime \prime}=0\right)$ vibrational bands are consistent with spectra reported by Berden et al., who were the first to observe the $\mathrm{k}^{3} \Pi(v=0)$ level in 1997 [142]. As indicated in Figure 3.10, the ${ }^{\mathrm{S}} \mathrm{R}_{32}(1)$ transition excites molecules to the $\Omega^{\prime}=2$ manifold, the ${ }^{\mathrm{R}} \mathrm{R}_{22}(1)$ and ${ }^{\mathrm{Q}} \mathrm{Q}_{22}(1)$ transitions in the $\Omega^{\prime}=1$ manifold, and the ${ }^{\mathrm{Q}} \mathrm{R}_{12}(1),{ }^{\mathrm{P}} \mathrm{Q}_{12}(1)$ and ${ }^{\mathrm{O}} \mathrm{P}_{12}(1)$ transitions in the $\Omega^{\prime}=0$ manifold of the $\mathrm{k}^{3} \Pi_{\Omega^{\prime}}$ state. Note that the ${ }^{\mathrm{R}} \mathrm{R}_{22}(1)$ and ${ }^{\mathrm{Q}} \mathrm{Q}_{22}(1)$ branches shown in Figure 3.10 exhibit a doublet-like structure for $\mathrm{k}^{3} \Pi(v=1,2,3)$, but not for $\mathrm{k}^{3} \Pi(v=0)$. The splitting is 1.0, 1.6 and $1.2 \mathrm{~cm}^{-1}$ for $v^{\prime}=1,2,3$, respectively, and has not been observed by Berden et al. The reason for the splitting is not clear.

If the splitting were due to a population transfer from the $\mathrm{a}^{3} \Pi_{1}(v=0, J=1,+)$ parity level to the - parity level, $\mathrm{k}^{3} \Pi$ states with + parity could be accessed. This would result in a splitting of lines if the lambda doubling of $\mathrm{k}^{3} \Pi_{1}$ can be resolved. In this scenario, however, additional lines were also expected to occur close to the $\Omega^{\prime}=0$ lines. Since $\mathrm{k}^{3} \Pi_{0}$ exhibits a larger lambda doubling than $\mathrm{k}^{3} \Pi_{1}$, Berden et al. were able to observe these additional lines because of collisional population transfer between the + and - parity levels of the $\mathrm{a}^{3} \Pi$ state. The $\mathrm{k}^{3} \Pi_{1}$ and $\mathrm{k}^{3} \Pi_{2}$ lambda splittings, however, are expected to be too small to be resolved. For experiments presented in the present 

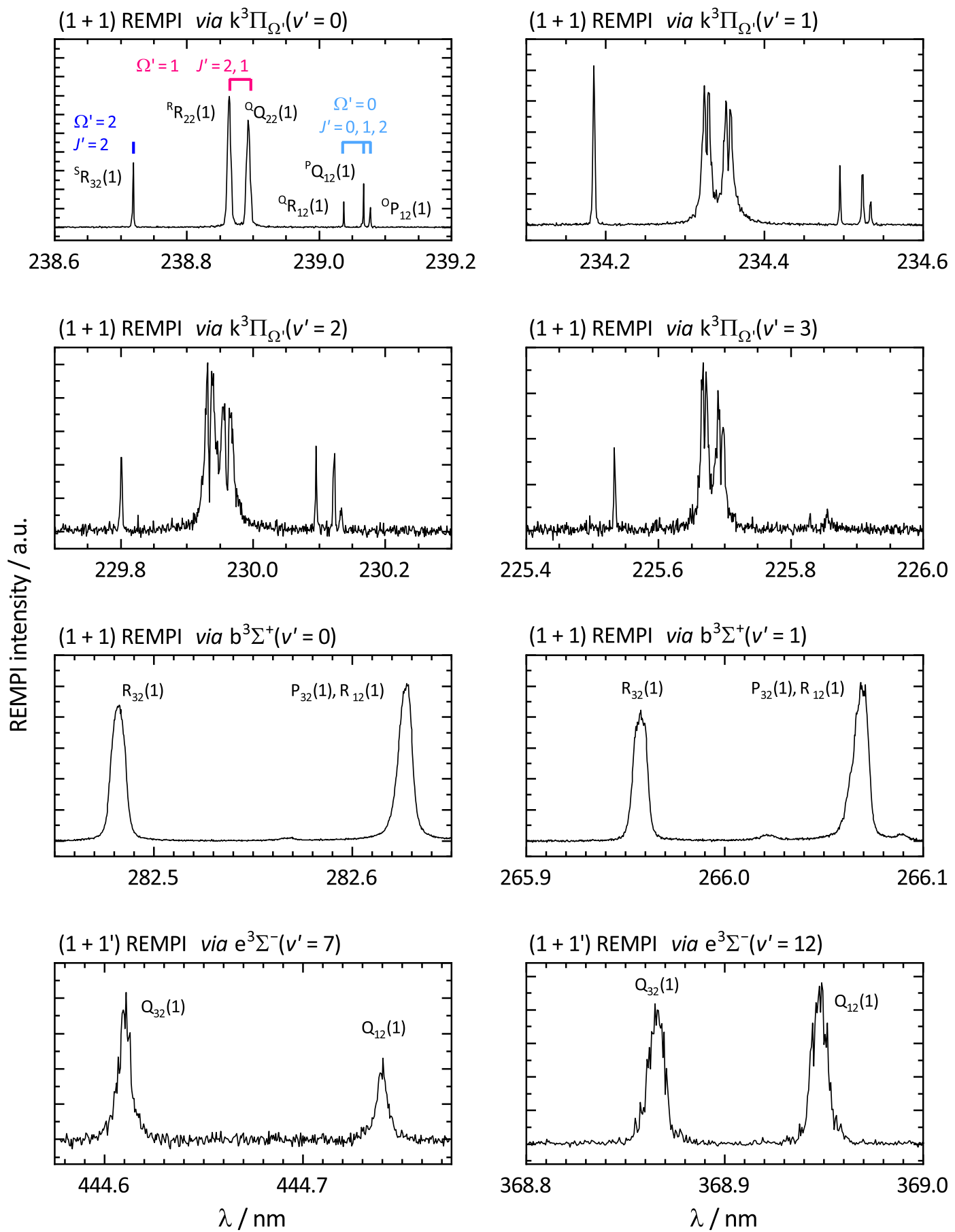

Figure 3.10: Various resonance-enhanced multi-photon ionization (REMPI) schemes for the detection of $\mathrm{CO} \mathrm{a}^{3} \Pi_{1}\left(v^{\prime \prime}=0, J^{\prime \prime}=1,+\right)$, which has been generated in advance from $\mathrm{X}^{1} \Sigma^{+}(v=0, J=1,-)$ by laser-exciting the $\mathrm{Q}_{2}(1)$ transition. In $\left(1+1^{\prime}\right)$ REMPI via $\mathrm{e}^{3} \Sigma^{-}\left(v^{\prime}=7\right)$ and $\mathrm{e}^{3} \Sigma^{-}\left(v^{\prime}=12\right)$, the ionizing laser is operated at $297.0 \mathrm{~nm}$ and $234.0 \mathrm{~nm}$, respectively. 
Table 3.2: Wavelengths in $\mathrm{nm}$ of REMPI transitions suited for the detection of $\mathrm{CO}$ $\mathrm{a}^{3} \Pi_{1}\left(v^{\prime \prime}=0, J^{\prime \prime}=1,+\right)$. Respective transitions are shown in Figure 3.10.

\begin{tabular}{llllllll}
\hline \hline State & Transition & $\boldsymbol{v}^{\prime}=\mathbf{0}$ & $\boldsymbol{v}^{\prime}=\mathbf{1}$ & $\boldsymbol{v}^{\prime}=\mathbf{2}$ & $\boldsymbol{v}^{\prime}=\mathbf{3}$ & $\boldsymbol{v}^{\prime}=\mathbf{7}$ & $\boldsymbol{v}^{\prime}=\mathbf{1 2}$ \\
\hline $\mathrm{k}^{3} \Pi$ & ${ }^{\mathrm{S}} \mathrm{R}_{32}(1)$ & 238.719 & 234.184 & 229.801 & 225.533 & - & - \\
& ${ }^{\mathrm{R}} \mathrm{R}_{22}(1)$ & 238.863 & 234.327 & 229.934 & 225.669 & - & - \\
& ${ }^{\mathrm{Q}} \mathrm{Q}_{22}(1)$ & 238.892 & 234.354 & 229.961 & 225.694 & - & - \\
& ${ }^{\mathrm{R}_{12}(1)}$ & 239.037 & 234.495 & 230.095 & 225.829 & - & - \\
& ${ }^{{ }} \mathrm{Q}_{12}(1)$ & 239.067 & 234.524 & 230.123 & 225.855 & - & - \\
& ${ } \mathrm{P}_{12}(1)$ & 239.077 & 234.534 & 230.134 & - & - & - \\
\hline $\mathrm{b}^{3} \Sigma^{+}$ & $\mathrm{R}_{32}(1)$ & 282.482 & 265.958 & - & - & - & - \\
& $\mathrm{P}_{32}(1), \mathrm{R}_{12}(1)$ & 282.627 & 266.069 & - & - & - & - \\
\hline $\mathrm{e}^{3} \Sigma^{-}$ & $\mathrm{Q}_{32}(1)$ & - & - & - & - & 444.610 & 368.863 \\
& $\mathrm{Q}_{12}(1)$ & - & - & - & - & 444.740 & 368.930 \\
\hline \hline
\end{tabular}

thesis, collisional population transfer can be excluded because laser-excitation of the beam is performed in the collision-free regime of the jet.

If the splitting were due to low- $J$ perturbations of the $\mathrm{k}^{3} \Pi_{1}(v=1,2,3)$ levels, the splitting should have been also observed by Berden et al., who detected vibrational states up to $\mathrm{k}^{3} \Pi(v=6)$.

If the splitting observed for $\mathrm{k}^{3} \Pi_{1}$ were due to first-order Stark splitting induced by electric fields of the detector setup, a splitting of $\mathrm{k}^{3} \Pi_{2}$ would be expected too. However, the $\mathrm{k}^{3} \Pi_{2}$ splitting is not observed in the spectra, although the first-order Stark effect of $\mathrm{k}^{3} \Pi_{2}$ should be even stronger than for $\mathrm{k}^{3} \Pi_{1}$ due to the higher $\Omega^{\prime}$. Furthermore, the splitting is not observed by Berden et al., who also excite the molecules in the presence of an electric field. If the observed vibrational state dependent splitting originates from Stark splitting in the electric field of the detector, which is approximately $500 \mathrm{~V} / \mathrm{cm}$, the resultant dipole moment of these states would be in the order of $10^{2} \mathrm{D}$, which appears to be unreasonably high for the $\mathrm{k}^{3} \Pi$ valence state. An alternative explanation could be the AC Stark effect (Autler-Townes effect) as the splitting seems to increase when the REMPI laser is focused. Further experiments probing the dependence of the splitting on electric field strength and laser power are clearly needed solve this issue. 


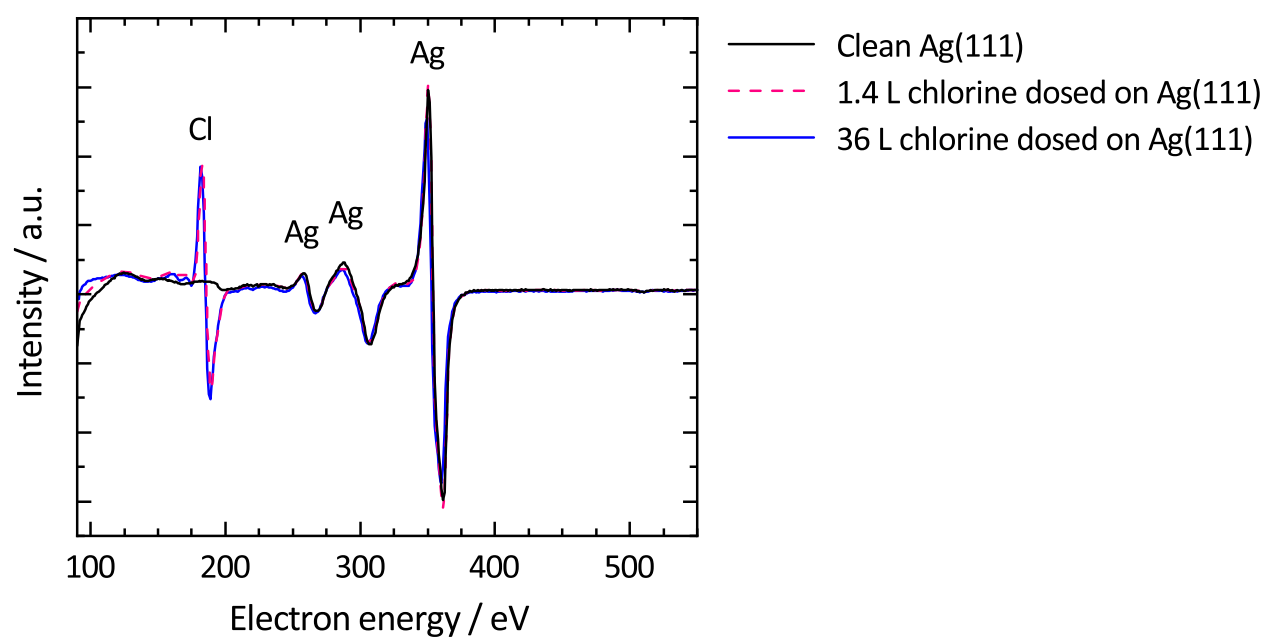

Figure 3.11: Auger electron spectra of an atomically clean (black solid) and a chlorinated $\operatorname{Ag}(111)$ surface. Spectra are shown for two different $\mathrm{Cl}_{2}$ dosages (red dashed, blue solid), indicating that the ratio between chlorine and silver peak intensities does not change for doses between $1.4 \mathrm{~L}$ and $36 \mathrm{~L}$. The surface temperature is kept at room temperature while dosing.

\subsubsection{Preparation of Chlorinated $\mathrm{Ag}(\mathbf{1 1 1})$}

In the literature, the controversial structure of chlorinated $\operatorname{Ag}(111)$ surfaces is discussed for different chlorine dosages and adsorption temperatures [143-147]. However, there is clear evidence that the surface work function increases by $1.5 \mathrm{eV}$ when $\mathrm{Ag}(111)$ is dosed with chlorine at room temperature [144]. Due to the strong increase in the work function, the chlorination technique is used to passivate the $\operatorname{Ag}(111)$ such that the vibrational relaxation of highly vibrationally excited $\mathrm{CO}$ is suppressed in surface scattering experiments.

Prior to chlorination, the $\mathrm{Ag}(111)$ surface is cleaned by argon ion sputtering and subsequent annealing at $870 \mathrm{~K}$, as described in Section 3.1. For chlorination, the surface is exposed to $5 \times 10^{-8}$ Torr of molecular chlorine for $5 \mathrm{~min}$. This corresponds to a dose of $15 \mathrm{~L}$. The surface temperature is kept at room temperature while dosing. The deposition of chlorine on the surface is monitored by Auger electron spectroscopy (AES). See Figure 3.11. The ratio between the chlorine and silver peak intensities initially increases with the amount of $\mathrm{Cl}_{2}$ dosed on the surface until it reaches a plateau where the ratio is invariant to continuing dosing (see Figure 1 in Reference [144]). Figure 3.11 demonstrates that the plateau is reached for doses between $1.4 \mathrm{~L}$ and $36 \mathrm{~L}$. Thus, the 


\section{Experimental}

work function of the chlorinated $\mathrm{Ag}(111)$ surfaces is expected to be $6.2 \mathrm{eV}$ when dosing $15 \mathrm{~L}$ of molecular chlorine.

Chlorination of $\operatorname{Ag}(111)$ inhibits the vibrational relaxation of directly scattered highly vibrationally excited CO. See Sections 4.3.2 and 5.3.1. Molecular beam-surface scattering experiments probing the energy transfer between molecule and surface require that incident $\mathrm{CO}$ molecules are not chemically converted at the chlorinated surface. Two observations indicate that $\mathrm{CO}$ is not reacting with chlorinated $\mathrm{Ag}(111)$. First, the REMPI spectra of surface scattered $\mathrm{CO}$ do not change with time, indicating that surface scattering conditions and surface composition are not affected by the exposure of the surface to the molecular beam. Second, AES spectra of the chlorinated surface after being exposed to the molecular beam for several hours confirm the absence of chemisorbed CO or conceivable reaction products. In contrast, the situation is different when $\operatorname{Ag}(111)$ is covered with oxygen atoms by dosing $\mathrm{NO}_{2}$ at $510 \mathrm{~K}$ [148-150]. Oxygen covered $\mathrm{Ag}(111)$ also suppresses vibrational relaxation, but the effect attenuates rapidly as the molecular beam impinges on the surface. Here, temperature programmed desorption experiments show that $\mathrm{CO}$ chemisorbs to the surface and reacts with oxygen by forming $\mathrm{CO}_{2}$.

\subsubsection{Surface Scattering Experiments}

This section summarizes the experimental conditions used in time-of-flight measurements, angular distribution measurements, and REMPI spectra measurements. All experiments have in common that highly vibrationally excited CO is generated $20-22 \mathrm{~mm}$ upstream from the surface. $\mathrm{CO}(v=17)$ is produced by two pump lasers and one dump laser, as described in Section 3.4.1. Corresponding wavelengths and pulse energies are given in Table 3.1. The beam diameter of the first pump laser is $3 \mathrm{~mm}$. The beam diameter of the second pump laser is narrowed down to $3 \mathrm{~mm}$ using a telescope consisting of two lenses with focal lengths $f=500 \mathrm{~mm}$ and $f=-100 \mathrm{~mm}$. The spot size of the dump laser is slightly larger than that of the second pump laser in order to minimize spurious fluorescence from remaining $\mathrm{CO}^{3} \Sigma^{-}(v=12)$.

REMPI ionized molecules are guided to the MCP detector. See for instance Figure 3.3. The outer lens, the inner lens, the repeller, and the grid are operated at $-200 \mathrm{~V},-900 \mathrm{~V}$, $+100 \mathrm{~V}$, and $-2000 \mathrm{~V}$, respectively. The MCP plates are operated between $-1450 \mathrm{~V}$ and

$-1950 \mathrm{~V}$. Note that the MCP voltage is ramped from $-1100 \mathrm{~V}$ to the operation voltage after the probe laser is fired in order to prevent saturation of the MCP detector by UV stray light. 
REMPI Spectra Measurements REMPI spectra are measured in order to determine vibrational state distributions and rotational state distributions of surface scattered molecules. $(1+1) \mathrm{A}^{1} \Pi\left(v^{\prime}, J^{\prime}\right) \leftarrow \mathrm{X}^{1} \Sigma^{+}\left(v^{\prime \prime}, J^{\prime \prime}\right)$ REMPI spectra covering the wavelength range between $228 \mathrm{~nm}$ and $245 \mathrm{~nm}$ probe the vibrational bands $\left(v^{\prime}-v^{\prime \prime}\right) 8-18,7-17,6$ 16, 9-18, 5-15, 8-17, 4-14, 7-16, 6-15, and 9-17. REMPI spectra of surface scattered molecules are probed $1 \mathrm{~mm}$ away from the surface. The MCP detector is operated at $1800 \mathrm{~V}$ or $-1850 \mathrm{~V}$ for the detection of scattered molecules and at $-1550 \mathrm{~V}$ or $-1650 \mathrm{~V}$ for the detection of incident molecules. The beam diameter of the probe laser is narrowed down to $2 \mathrm{~mm}$ using a telescope consisting of two lenses with focal lengths of $f=200 \mathrm{~mm}$ and $f=-100 \mathrm{~mm}$.

Time-of-Flight Measurements Time-of-flight measurements are performed for both incident and scattered molecules. For the incoming beam, the $\mathrm{R}(0)$ branch of the 817 band is probed at $233.91 \mathrm{~nm}$. The probe laser beam is focused by a $f=500 \mathrm{~mm}$ lens. The MCP is operated at $-1450 \mathrm{~V}$ or $-1550 \mathrm{~V}$. Arrival time distributions of incident CO are measured $3.3 \mathrm{~mm}, 5.3 \mathrm{~mm}, 7.3 \mathrm{~mm}, 9.3 \mathrm{~mm}, 11.3 \mathrm{~mm}$, and $13.3 \mathrm{~mm}$ upstream from the surface. For the scattered beam, the MCP detector is operated at $-1900 \mathrm{~V}$. Scattered molecules are detected at a surface distance of $4.3-5.3 \mathrm{~mm}$. The probe laser beam is focused by a $f=500 \mathrm{~mm}$ lens. The $4-14,5-15,6-16$, and $8-17$ vibrational band heads are probed at $233.32 \mathrm{~nm}, 235.72 \mathrm{~nm}, 238.23 \mathrm{~nm}$, and $233.86 \mathrm{~nm}$, respectively. $\mathrm{R}(15), \mathrm{Q}(25), \mathrm{P}(36)$, and $\mathrm{Q}(37)$ rotational transitions of the 7-17 band are probed at $240.91 \mathrm{~nm}, 241.62 \mathrm{~nm}, 242.98 \mathrm{~nm}$, and $242.46 \mathrm{~nm}$, respectively. $\mathrm{Q}(6), \mathrm{P}(14)$, and $\mathrm{P}(27)$ rotational transitions of the $8-17$ band are probed at $233.98 \mathrm{~nm}, 234.39 \mathrm{~nm}$, and $235.22 \mathrm{~nm}$, respectively.

Angular Distribution Measurements In angular distribution measurements, the probe laser is parked $4.3-5.3 \mathrm{~mm}$ away from the surface. The probe laser is focused by a $f=500 \mathrm{~mm}$ lens. The MCP detector is operated at $-1900 \mathrm{~V}$. The $z$ position of the probe laser is varied in total by $7.62 \mathrm{~mm}$ around the center of the molecular beam in steps of $0.51 \mathrm{~mm}$. Measurements on the incoming beam show that the beam diameter of the incident molecular beam is $1 \mathrm{~mm}$. Due to experimental limitations, the surface normal of the $\mathrm{Au}(111)$ and the $\mathrm{Ag}(111)$ surface is tilted by $5^{\circ}$ and $3^{\circ}$, respectively, with respect to the incoming molecular beam axis. Angular distributions are recorded for $\mathrm{CO}(v=17)$ scattered into $v_{\text {sca }}=17$ and $v_{\text {sca }}=14$. Corresponding wavelengths probing the $4-14$ and $8-17$ band heads are $233.32 \mathrm{~nm}$ and $233.86 \mathrm{~nm}$. 


\subsection{Chemicals}

Table 3.3 provides an overview of chemical compounds used in experiments presented in the present thesis.

\begin{tabular}{llll}
\hline \hline Compound & CAS Registry Number & Manufacturer & Purity \\
\hline Argon & $7440-37-1$ & Linde & $99.999 \%$ \\
Carbon monoxide & $630-08-0$ & Air Liquide & $99.997 \%$ \\
Chlorine & $7782-50-5$ & Linde & $99.8 \%$ \\
Coumarin C460 & $91-44-1$ & Exciton & \\
Coumarin C480 & $41267-76-9$ & Exciton & \\
DABCO & $280-57-9$ & Sigma-Aldrich & $\geq 99 \%$ \\
Ethanol & $64-17-5$ & Sigma-Aldrich & $99.9 \%$ \\
Hydrogen & $1333-74-0$ & Air Liquide & $99.999 \%$ \\
Methanol & $67-56-1$ & VWR International & $99.9 \%$ \\
Neon & $7440-01-9$ & Westfalen AG & $99.99 \%$ \\
Nitrogen & $7727-37-9$ & Air Liquide & $99.999 \%$ \\
Nitrogen dioxide & $10102-44-0$ & Air Liquide & $98 \%$ \\
Rhodamine 6G & $989-38-8$ & Exciton & \\
Styryl 8 (LDS 751) & - & Exciton & \\
Xenon & $7440-63-3$ & Air Liquide & $99.998 \%$ \\
\hline \hline
\end{tabular}

Table 3.3: Information about chemical compounds. 


\section{Data Analysis}

\subsection{Time-of-Flight Distributions}

Molecular beam-surface scattering experiments probing the dynamics of molecules at surfaces require precise knowledge about the initial and final energetic states of the molecules involved. This includes the knowledge about the translational energy of the molecules. The present section deals with the analysis of time-of-flight distributions, from which velocity distributions and mean translational energies of molecular beams can be derived. The analysis is described first for incident molecules and then for scattered molecules.

The velocity of a single molecule is defined by the rate at which the molecule changes its position with respect to time. In order to determine the incidence translational energy, the arrival time of the incoming beam is measured for various $x$ positions along the propagation direction of the molecular beam. The first well-defined point in space and time is the one at which highly vibrationally excited $\mathrm{CO}$ is prepared. It is characterized by the spacial position $x_{\text {prep }}$ of the dump laser beam, which intersects the molecular beam perpendicularly, and the dump laser timing $t_{\text {prep. }}$. The molecular beam can be considered to be stationary during the interaction time with the dump laser because the laser pulse duration is in the order of a few ns. Thus, the dump laser only excites molecules located in the volume of the $1 \mathrm{~mm}$ wide laser beam centered at $x_{\text {prep. This }}$ corresponds to a fraction of the initial beam produced by the nozzle. The package of highly vibrationally excited molecules consists of molecules with a certain spatial spread and velocity spread. Thus, a time-of-flight distribution is measured when probing the beam further downstream. This is achieved by scanning the probe laser timing $t_{\text {inc }}$ for a certain probe laser focus position $x_{\text {inc }}$ which can be arbitrarily varied between the surface position $x_{\text {surf }}$ and $x_{\text {prep. }}$.

The incidence translational energy can be determined in two different ways with similar accuracy. The first method requires the measurement of time-of-flight distributions for different probe laser positions $x_{\text {inc }}$. These distributions are illustrated in Panel (A) of Figure 4.1. The timing $t_{\text {inc, max }}$ at which the most intense part of the molecular beam appears is calculated by fitting a Gaussian function to each data set. Here, $t_{\text {inc, max }}$ as 
(A)

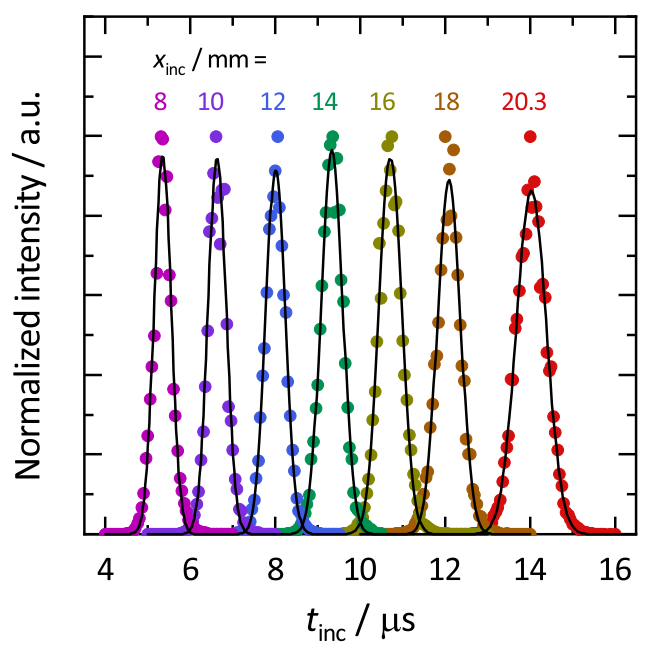

(B)

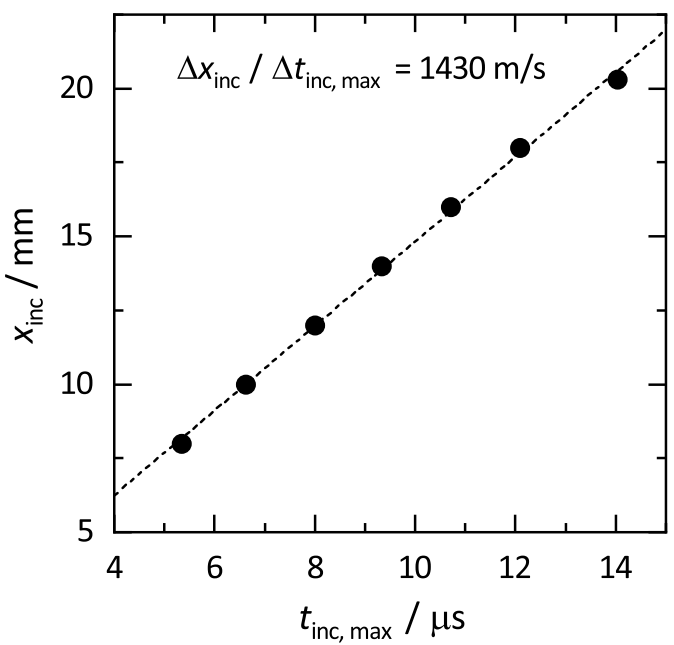

Figure 4.1: Panel (A) shows background-corrected time-of-flight distributions of incident highly vibrationally excited $\mathrm{CO}$ for seven different probe laser positions $x_{\text {inc }}$. Panel (B) shows a plot of $x_{\text {inc }}$ versus the position of the distributions maxima $t_{\text {inc, max }}$. The velocity of the incident beam $v_{\text {inc }}$ is given by the slope of the linear fit (dashed line). In this example, $v_{\text {inc }}$ is $1430 \mathrm{~m} / \mathrm{s}$, which corresponds to a translational energy of $0.30 \mathrm{eV}$.

well as the amplitude and the width of the distribution are fitting parameters. A plot of $x_{\text {inc }}$ against $t_{\text {inc, max }}$ yields a straight line with a slope equal to the velocity of the incoming beam. See Panel (B) of Figure 4.1.

In an alternative approach, the velocity distribution of the particles in the molecular beam is extracted from the measured time-of-flight distribution. It will be shown that the mean velocity of the beam can be derived from this velocity distribution. A floating three-dimensional Maxwellian velocity distribution of the flux $f_{\mathrm{f}}(v)$ is commonly used to describe the speed distribution of supersonic molecular beams [151].

$$
f_{\mathrm{f}}(v)=N_{v} v^{3} \exp \left[-\left(\frac{v-v_{0}}{w_{v}}\right)^{2}\right]
$$

Here, $v$ is the velocity, $v_{0}$ is the center of the exponential part of the distribution, $w_{v}$ is a measure of the distribution's width, and $N_{v}$ is the normalization factor. The goal is to convert Equation 4.1 into a flight time distribution $f(t)$ that can be used to fit the experimental data.

In general, the flux is defined as the number of molecules passing through a unit area 
per unit time. However, the measured REMPI signal is proportional to the number of molecules in the volume of the probe laser focus, i.e. the density of molecules in the molecular beam at the probe laser focus position. Equation 4.1 can be converted into a density distribution $f(v)$ by dividing the flux of particles by their velocity. Note that the flux-to-density conversion should be applied twice because both the laser preparation and the laser detection of highly vibrationally excited CO need to be taken into account.

$$
f(v)=\frac{1}{v^{2}} f_{\mathrm{f}}(v)
$$

The transformation from velocity to time space is achieved by multiplying Equation 4.2 with the Jacobian.

$$
f(t)=f(v) \frac{\mathrm{d} v}{\mathrm{~d} t}
$$

Since the velocity $v$ of a molecule is defined as

$$
v=\frac{l}{t}
$$

where $l$ is the distance covered by the molecule during the time $t$, the final expression for $f(t)$ is yielded by combining Equations 4.1, 4.2, 4.3, and 4.4.

$$
\begin{gathered}
f(t)=\frac{\mathrm{d} v}{\mathrm{~d} t} \frac{1}{v^{2}} f_{\mathrm{f}}(v) \\
f(t)=N_{v}\left(-\frac{l}{t^{2}}\right)\left(\frac{t}{l}\right)^{2}\left(\frac{l}{t}\right)^{3} \exp \left[-\left(\frac{l / t-v_{0}}{w_{v}}\right)^{2}\right]
\end{gathered}
$$

In order to determine the mean velocity of the incoming beam, a single time-of-flight distribution needs to be measured, preferably as far away from $x_{\text {prep }}$ as possible. The molecules then have traveled the distance $l_{\text {inc }}=x_{\text {prep }}-x_{\text {inc }}$. When $l$ is replaced by the constant $l_{\text {inc }}$, Equation 4.6 can be fitted to the experimental time-of-flight trace. Note that the time $t$ corresponds to the time delay between the dump laser and the probe laser. $t$ is varied in the experiment by shifting the probe laser timing.

Having determined the fitting parameters $N_{v}, w_{v}$, and $v_{0}$, the mean velocity of the incoming beam $\left\langle v_{\text {inc }}\right\rangle$ can be calculated as

$$
\left\langle v_{\text {inc }}\right\rangle=\int_{0}^{\infty} v f_{\mathrm{f}}(v) \mathrm{d} v .
$$

The mean value of the squared velocity is given by

$$
\left\langle v_{\text {inc }}^{2}\right\rangle=\int_{0}^{\infty} v^{2} f_{\mathrm{f}}(v) \mathrm{d} v,
$$




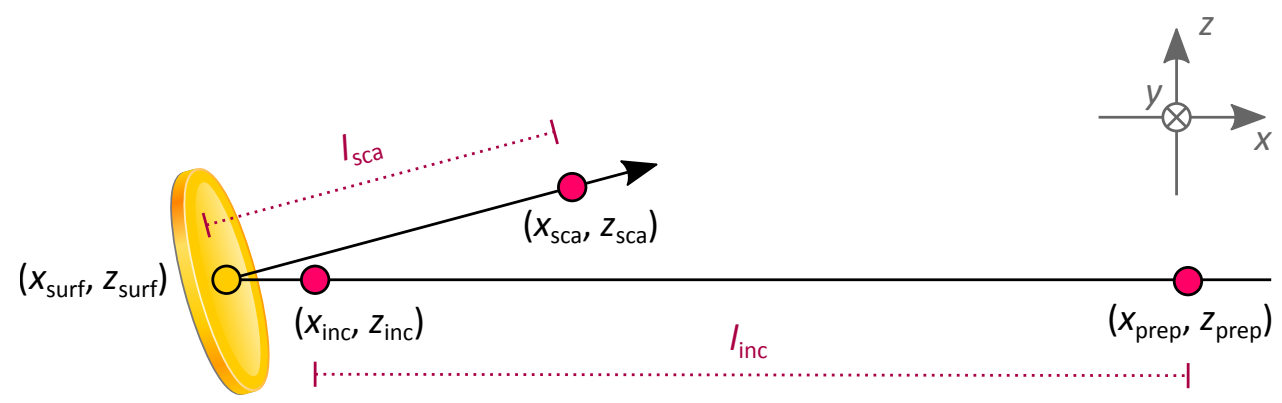

Figure 4.2: Schematic drawing of the time-of-flight setup. The molecular beam is indicated by the black arrow. Laser beam positions (red points) are shown for the preparation laser ("prep") and the probe laser, which either crosses the incoming beam ("inc") or the scattered beam ("sca"). The surface position is indicated by the yellow point.

which is needed in order to calculate the mean translational energy as

$$
\left\langle E_{\text {inc }}\right\rangle=\frac{1}{2} m\left\langle v_{\text {inc }}^{2}\right\rangle,
$$

where $m$ is the mass of the molecule.

The mean velocity of the scattered beam $\left\langle v_{\text {sca }}\right\rangle$ is derived in a similar fashion. Again, Equation 4.6 is used for fitting the experimentally measured time-of-flight distribution. Now, the time $t$ corresponds to the time that is needed for the molecules to travel from the surface position $\left(x_{\text {surf }}, z_{\text {surf }}\right)$ to the point of detection $\left(x_{\text {sca }}, z_{\text {sca }}\right)$. The time at which the molecules leave the surface is approximately given by the arrival time at the surface assuming that the interaction time at the surface is several orders of magnitude shorter than the flight time to the detection zone. The arrival time at the surface can be calculated since the surface position and the incoming beam velocity are known. Due to the fact that the surface is slightly tilted, $x$ and $z$ coordinates must be taken into account for the calculation of the flight distance $l_{\text {sca }}=\sqrt{\left(x_{\text {surf }}-x_{\text {sca }}\right)^{2}+\left(z_{\text {surf }}-z_{\text {sca }}\right)^{2}}$. See Figures 4.2 and 4.3 for clarification.

\subsection{Angular Distributions}

Angular distributions of scattered molecular beams help to identify the scattering mechanism in reactive and non-reactive molecule-surface scattering. In non-reactive scattering, angular distributions provide valuable information on whether the molecules are directly scattered, which is indicated by a narrow distribution, or whether they undergo 


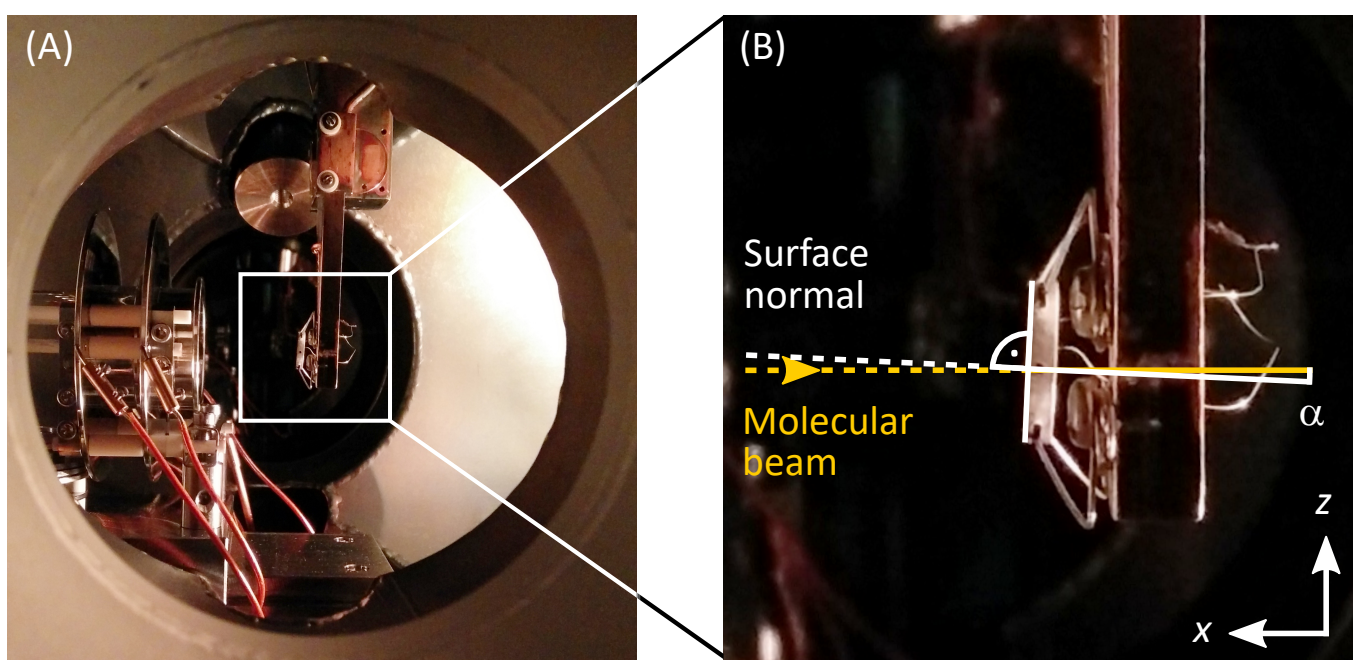

Figure 4.3: Panel (A) shows the surface scattering chamber. The ring-shaped electrodes of the detector, through which the molecular beam approaches the surface, are located on the left-hand side. The surface holder and the surface itself are shown sideways in the center of the picture. A magnified image of the surface holder and the surface is shown in Panel (B), in which the incoming molecular beam axis, the surface normal, and the surface tilt angle $\alpha$ are sketched.

trapping-desorption, indicated by a broad distribution.

The width of the scattered molecular beam is probed by parking the probe laser at a certain $x$ position, $x_{\text {sca }}$, and detecting the scattered molecules for various $z$ positions of the probe laser, $z_{\text {sca. }}$. The experimental geometry is described in Section 3.4.4. The scattering angle $\theta_{\text {sca }}$ is defined as the angle between the propagation direction of scattered molecules and the surface normal. $\theta_{\text {sca }}$ can be calculated from the position at which the incident beam strikes the surface, $\left(x_{\text {surf }}, z_{\text {surf }}\right)$, and the probe laser position.

$$
\theta_{\text {sca }}=\arctan \left(\frac{z_{\text {sca }}-z_{\text {surf }}}{x_{\text {sca }}-x_{\text {surf }}}\right)-\alpha
$$

$z_{\text {surf }}$ equals the $z$ position of the incoming beam $z_{\text {inc }}$. Note that the scattering angle needs to be corrected by $\alpha$, the angle about which the surface is tilted with respect to the incoming molecular beam axis. This surface tilt is illustrated in Figure 4.3. $\alpha$ is $5^{\circ}$ in experiments with $\mathrm{Au}(111)$ and $3^{\circ}$ in experiments with $\mathrm{Ag}(111)$.

The measured REMPI intensity must be corrected for the spatial detection efficiency of the detector. The correction factor is determined by leaking $1.1 \times 10^{-8}$ Torr CO 
into the vacuum chamber and measuring the $(2+1)$ REMPI signal via the $\mathrm{B}^{1} \Sigma^{+}\left(v^{\prime}=\right.$ $0) \leftarrow \mathrm{X}^{1} \Sigma^{+}\left(v^{\prime \prime}=0\right)$ transition for the same probe laser positions as used in the angular distribution experiment. Since the number of ions should be the same at every position, variations in the measured REMPI intensity arise from different spatial detection efficiencies.

The final angular distribution is characterized by plotting the corrected REMPI intensity $I$ as a function of $\theta_{\text {sca }}$ and by fitting the $\cos ^{n}$ distribution

$$
I\left(\theta_{\text {sca }}\right)=I_{0} \cos ^{n}\left(\theta_{\text {sca }}-\theta_{0}\right)
$$

to these data points. Here, $I_{0}$ is the normalized intensity and $\theta_{0}$ is the maximum of the distribution. The exponent $n$ describes the width of the distribution. The angular distribution of trapped molecules desorbing from the surface resembles a cosine distribution with $n=1$. Directly scattered molecules show a much narrower distribution with $n>1$. In addition, directly scattered molecules obey the "memory effect", which means that the angle of the scattered beam depends on the incidence angle $\theta_{\text {inc. Note }}$ that $\theta_{0}$ is expected to be slightly larger than $\left|\theta_{\text {inc }}\right|=|\alpha|$, assuming that the molecules lose momentum parallel to the surface normal whereas their momentum perpendicular to the surface normal is conserved.

\subsection{Vibrational State Distributions}

Highly vibrationally excited $\mathrm{CO} \mathrm{X}^{1} \Sigma^{+}\left(v_{\text {inc }}^{\prime \prime}=17\right)$ may lose vibrational quanta during the collision with a metal surface. The aim of this section is to determine the final vibrational population distribution in the scattered beam. This can be achieved by analyzing the $(1+1)$ REMPI spectra via the $\mathrm{A}^{1} \Pi\left(v^{\prime}\right) \leftarrow \mathrm{X}^{1} \Sigma^{+}\left(v^{\prime \prime}\right)$ transition, which consist of several vibrational bands $v^{\prime}-v^{\prime \prime}$ probing different $v^{\prime \prime}$. First, the quantitative analysis of the REMPI spectra is described, which yields the relative population of each vibrational state. Second, the calculation of normalized vibrational populations is described, which are plotted in final vibrational state distributions. In addition, normalized vibrational populations are used to determine vibrational relaxation probabilities. The following analysis is based on the methodology that was developed for the analysis of REMPI spectra of highly vibrationally excited NO [86] and has been described explicitly for the scattering of $\mathrm{CO}(v=17)$ in References [31, 32].

\subsubsection{Relative Vibrational Populations}

The REMPI spectrum of surface scattered CO covers the wavenumber range between $40800 \mathrm{~cm}^{-1}$ and $43800 \mathrm{~cm}^{-1}$ and consists of several vibrational bands probing the vi- 
brational levels $v^{\prime \prime}=14,15,16,17$ and 18. The integrated REMPI signal of a certain vibrational band $v^{\prime}-v^{\prime \prime}$, which consists of many rotational lines, is related to the population of $v^{\prime \prime}$. The relative population of each $v^{\prime \prime}$ is calculated for a certain $v^{\prime}-v^{\prime \prime}$ band as

$$
\begin{aligned}
N_{v^{\prime}-v^{\prime \prime}}^{\text {sca }} \propto\left(\int_{v^{\prime}-v^{\prime \prime}} \frac{S_{\text {dump on }}(\tilde{\nu})}{P_{\text {probe }}(\tilde{\nu})} \mathrm{d} \tilde{\nu}-\beta \int_{v^{\prime}-v^{\prime \prime}} \frac{S_{\text {dump off }}(\tilde{\nu})}{P_{\text {probe }}(\tilde{\nu})} \mathrm{d} \tilde{\nu}\right) \gamma_{v^{\prime}-v^{\prime \prime}}^{-1} \\
\times\left\langle v_{\text {inc }}\right\rangle\left\langle v_{\text {sca }}\right\rangle w_{\text {ang }} w_{v} \Gamma(U)^{-1} .
\end{aligned}
$$

$S_{\text {dump on }}(\tilde{\nu})$ and $S_{\text {dump off }}(\tilde{\nu})$ are the REMPI signals of surface scattered CO for the dump laser turned on and turned off, respectively, $P_{\text {probe }}(\tilde{\nu})$ is the probe laser power, $\beta$ is the FCP depletion factor, $\gamma_{v^{\prime}-v^{\prime \prime}}$ is the band-specific detection efficiency, $\left\langle v_{\text {inc }}\right\rangle$ is the mean velocity of the incoming beam, $\left\langle v_{\text {sca }}\right\rangle$ is the mean velocity of the scattered beam, $w_{\text {ang }}$ is the width of the angular distribution, $w_{v}$ is the width of the velocity distribution of the scattered beam, and $\Gamma(U)$ is the gain of the MCP detector as a function of the MCP voltage $U .{ }^{1}$ In the following, Equation 4.12 is explained in more detail.

$S_{\text {dump on }}(\tilde{\nu})$ is the REMPI signal measured when the dump laser is turned on, stimulating emission into $\mathrm{X}^{1} \Sigma^{+}\left(v^{\prime \prime}=17, J^{\prime \prime}=0\right) . \quad S_{\text {dump on }}(\tilde{\nu})$ is divided by the probe laser power $P_{\text {probe }}(\tilde{\nu})$ to correct for fluctuations in the probe laser power. $P_{\text {probe }}(\tilde{\nu})$ is measured on-line while scanning the probe laser frequency. As shown in Panel (A) of Figure 4.4, the REMPI intensity scales linearly with $P_{\text {probe }}(\tilde{\nu})$, indicating that the first step of the $(1+1)$ REMPI process is saturated. The corrected REMPI signal is then integrated over the $v^{\prime}-v^{\prime \prime}$ band.

Even though the dump laser populates a single rovibrational state, the population of $v^{\prime \prime}$ states other than 17 by Frank-Condon pumping (FCP) cannot be avoided. This causes an undesired background signal because of two reasons. First, vibrational states lower than $v^{\prime \prime}=17$, which are the expected scattering products, are already present in the incident beam. Second, vibrational states higher than $v^{\prime \prime}=17$ are present in the incoming beam and will also lead to vibrationally relaxed scattering products. In order to quantify the background signal, the REMPI spectrum $S_{\text {dump off }}(\tilde{\nu})$ of surface scattered $\mathrm{CO}$ is measured with the dump laser turned off. The REMPI signal then originates only from Franck-Condon pumped highly vibrationally excited CO.

Note that the integrated background signal must be corrected by the depletion factor $\beta$ because the fluorescence is reduced when the dump laser is turned on. The dump laser depopulates the upper state from which fluorescence occurs because it drives the

\footnotetext{
${ }^{1}$ Note that $\gamma_{v^{\prime}-v^{\prime \prime}}$ and $\Gamma(U)$ are reciprocally defined compared to the formula presented in the Supporting Information of Reference [31].
} 
(A)

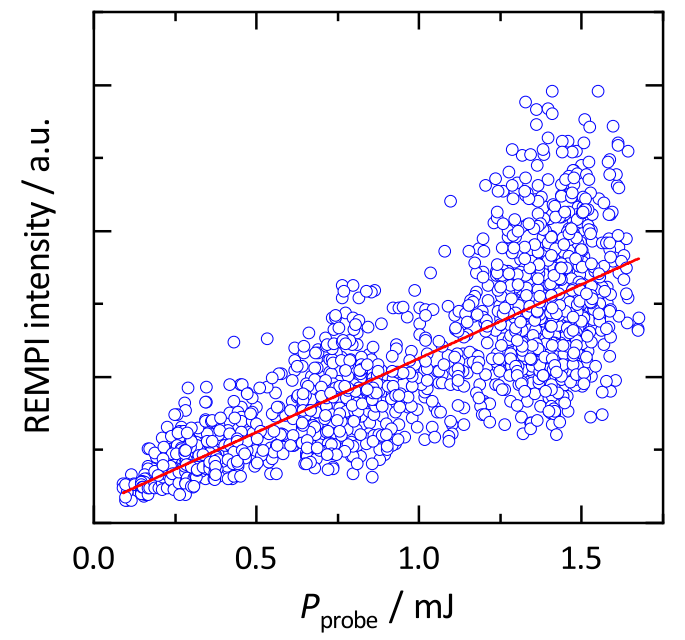

(B)

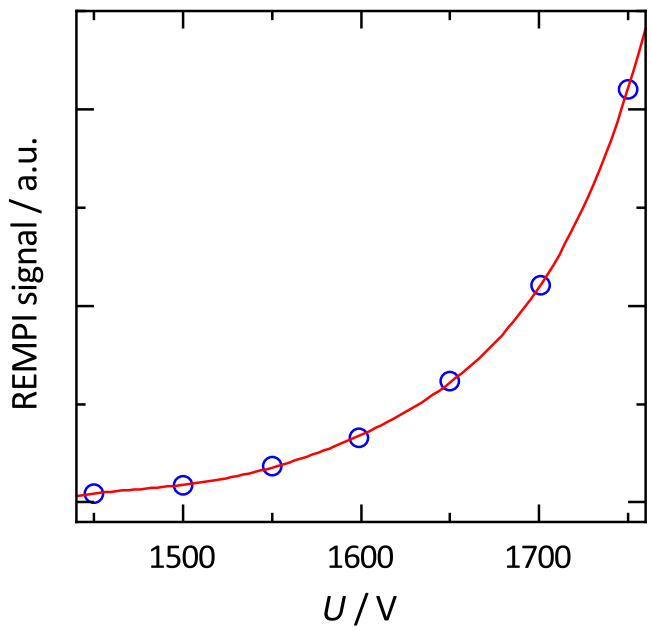

Figure 4.4: In Panel (A), the REMPI intensity of the $\mathrm{A}^{1} \Pi\left(v^{\prime}=8, J^{\prime}=1\right) \leftarrow$ $\mathrm{X}^{1} \Sigma^{+}\left(v^{\prime \prime}=17, J^{\prime \prime}=0\right)$ transition is plotted as a function of the probe laser pulse energy $P_{\text {probe }}$. The red line is a linear fit through the data points. Panel (B) shows the magnitude of the REMPI ion signal for different voltages of the MCP detector. The fit (red curve) corresponds to the function $\Gamma(U)$ used in Equation 4.12.

transition to $\mathrm{X}^{1} \Sigma^{+}\left(v^{\prime \prime}=17, J^{\prime \prime}=0\right)$. Panel $(\mathrm{A})$ in Figure 4.5 shows the depletion of the 7-16 band when the dump laser is turned on, and Panel (B) illustrates the population increase in $\mathrm{X}^{1} \Sigma^{+}\left(v^{\prime \prime}=17, J^{\prime \prime}=0\right)$ due to dumping. $\beta$ can be derived from REMPI spectra as shown in Panel (A) where $v^{\prime}-v^{\prime \prime}$ bands are detected prior to surface scattering. $\beta$ is obtained by dividing the integrated signal for dump on by the integrated signal for dump off. $\beta$ may vary from experiment to experiment and reflects the efficiency of dumping.

Panel (C) in Figure 4.5 shows the spectra of surface scattered CO for dump on and dump off. The REMPI signal that only probes the scattering products of incident CO in the single rovibrational state $\mathrm{X}^{1} \Sigma^{+}\left(v^{\prime \prime}=17, J^{\prime \prime}=0\right)$ corresponds to the difference between the black and the red trace. Its magnitude is given by the term in parentheses in Equation 4.12.

The signal is then divided by the band-specific detection efficiency $\gamma_{v^{\prime}-v^{\prime \prime}} \cdot \gamma_{v^{\prime}-v^{\prime \prime}}$ is determined experimentally by probing the vibrational bands $v^{\prime}-v^{\prime \prime}$ prior to surface scattering with the dump laser turned off. High $v^{\prime \prime}$ states are then populated by fluorescence and their population distribution is determined by the Einstein $A$ coefficients. Dividing 
(A)

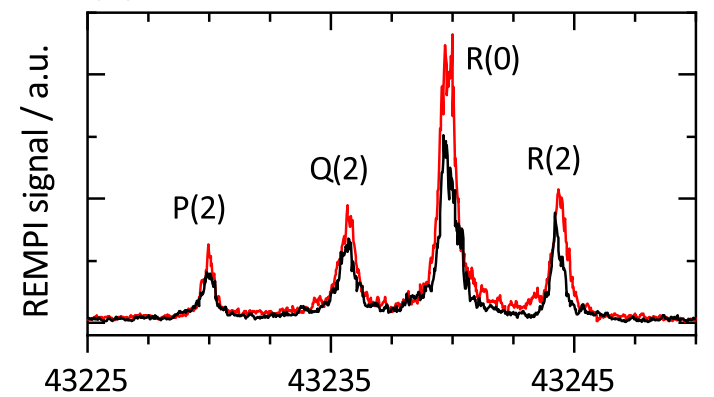

(C)
(B)

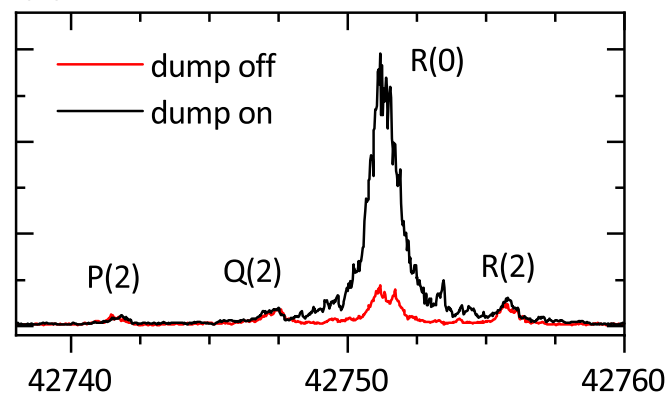



Figure 4.5: $(1+1) \mathrm{A} \leftarrow \mathrm{X}$ REMPI spectra of highly vibrationally excited $\mathrm{CO}$ are shown for dump on (black) and dump off (red). Panel (A) shows the 7-16 band of incident $\mathrm{CO}$ illustrating that the population in $v^{\prime \prime}=16$ is depleted when dumping. Panel (B) shows the 8-17 band of incident CO illustrating that the population in $\mathrm{X}^{1} \Sigma^{+}\left(v^{\prime \prime}=17, J^{\prime \prime}=0\right)$ is significantly increased when dumping. Panel (C) shows the spectrum of $\mathrm{CO}$ scattered from $\mathrm{Au}(111)\left(E_{\text {inc }}=0.57 \mathrm{eV}\right)$, where the red trace corresponds to the background signal induced by Franck-Condon pumping. Note that the intense $(2+1) \mathrm{B}^{1} \Sigma^{+}\left(v^{\prime}=0,1\right) \leftarrow \mathrm{X}^{1} \Sigma^{+}\left(v^{\prime \prime}=0,1\right)$ REMPI bands at $43460 \mathrm{~cm}^{-1}$ and $43428 \mathrm{~cm}^{-1}$ are removed from the spectrum.

the integrated spectrum of a certain $v^{\prime}-v^{\prime \prime}$ band by the corresponding Einstein $A$ coefficient gives $\gamma_{v^{\prime}-v^{\prime \prime}}$. Einstein $A$ coefficients of $\mathrm{A} \rightarrow \mathrm{X}$ vibronic transitions are calculated using the computer programs RKR1 2.0 [152] and LEVEL 8.0 [153]. Since the $v^{\prime}-v^{\prime \prime}$ REMPI spectra of both incident and scattered CO are measured directly after one another, $\gamma_{v^{\prime}-v^{\prime \prime}}$ accounts not only for vibronic transition intensities but also corrects for temporal shifts in the power of the pump lasers.

As described in Section 4.1, the density-to-flux conversion is achieved by multiplying 


\section{Data Analysis}

the signal by $\left\langle v_{\text {inc }}\right\rangle$ and $\left\langle v_{\text {sca }}\right\rangle$, accounting for both laser preparation and laser detection of highly vibrationally excited CO.

Since the scattered beam spreads along the $x, y$, and $z$ direction when it leaves the surfaces, REMPI signals must in general be corrected for the angular and longitudinal spread by multiplication with the width at half maximum of the angular distribution $w_{\text {ang }}$ and of the time-of-flight distribution $w_{v}$. However, this correction was found to be of minor importance because the scattered molecules are detected extremely close to the surface where the spatial spread of the scattered beam barely deviates from that of the incoming beam.

When vibrational bands are detected at different voltages of the MCP detector, the signal must be corrected for the gain of the detector. This is achieved by dividing the signal by $\Gamma(U) . \Gamma(U)$ is determined experimentally and reflects the magnitude of the REMPI signal as a function of MCP voltage. $\Gamma(U)$ is shown in Panel (B) of Figure 4.4.

\subsubsection{Vibrational Relaxation Probabilities}

Once $N_{v^{\prime}-v^{\prime \prime}}^{\mathrm{sca}}$ is known from Equation 4.12 for each vibrational band $v^{\prime}-v^{\prime \prime}$, the relative population of a certain $v^{\prime \prime}, N_{v^{\prime \prime}}^{\mathrm{sca}}$, is given by the mean value of $N_{v^{\prime}-v^{\prime \prime}}^{\mathrm{sca}}$ over all $v^{\prime}$ that probe this particular state. In the following, the determination of normalized vibrational populations and vibrational relaxation probabilities are described first for gold, then for silver.

As can be seen in Panel (C) of Figure 4.5, a rather small fraction of molecules populate $v^{\prime \prime}=14$ after $\mathrm{CO}\left(v^{\prime \prime}=17\right)$ is scattered from $\mathrm{Au}(111)$. The majority of molecules either scatter vibrationally elastically or relax to $v^{\prime \prime}=15$ and 16 . Assuming that the molecules do not relax to states lower than $v^{\prime \prime}=14$, the total number of scattered molecules $N_{\text {sca }}$ is given by summing $N_{v^{\prime \prime}}^{\text {sca }}$ from $v^{\prime \prime}=14$ to 17 . The normalized population of $v^{\prime \prime}, R_{v^{\prime \prime}}^{\text {sca }}$, is then calculated as

$$
R_{v^{\prime \prime}}^{\mathrm{sca}}=\frac{N_{v^{\prime \prime}}^{\mathrm{sca}}}{N_{\mathrm{sca}}}=\frac{N_{v^{\prime \prime}}^{\mathrm{sca}}}{\sum_{v^{\prime \prime}=14}^{17} N_{v^{\prime \prime}}^{\mathrm{sca}}}
$$

The vibrational relaxation probability $P_{\text {relax }}$ is given by

$$
P_{\text {relax }}=1-R_{17}^{\text {sca }}
$$

In order to test the assumption that the scattered molecules do not occupy vibrational levels other than $14 \leq v^{\prime \prime} \leq 17$, the ratio between the total number of scattered molecules $N_{\text {sca }}$ and the total number of incident molecules $N_{\text {inc }}$ is calculated.

$$
\frac{N_{\text {sca }}}{N_{\text {inc }}}=\frac{\sum_{v^{\prime \prime}=14}^{17} N_{v^{\prime \prime}}^{\text {sca }}}{N_{17}^{\text {inc }}}
$$


(A) $\mathrm{Ag}(111)$

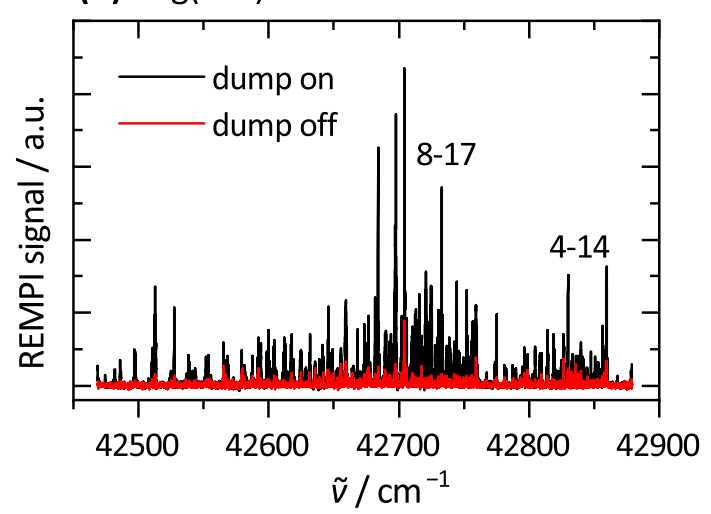

(B) Chlorinated $\mathrm{Ag}(111)$

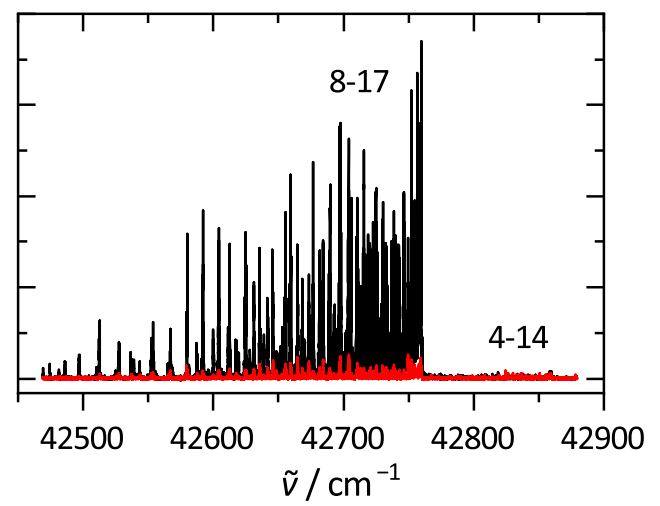

Figure 4.6: $(1+1) \mathrm{A} \leftarrow \mathrm{X}$ REMPI spectra of highly vibrationally excited $\mathrm{CO}$ are shown for dump on (black) and dump off (red). Panel (A) shows the 8-17 and the 4-14 band for $\mathrm{CO}$ scattered from $\mathrm{Ag}(111)$. Panel (B) shows spectra in the same spectral range for $\mathrm{CO}$ scattered from a chlorinated $\mathrm{Ag}(111)$ surface. In both cases, $E_{\text {inc }}=0.57 \mathrm{eV}$.

The number of incident molecules equals the number of molecules prepared in $v^{\prime \prime}=17$, $N_{17}^{\text {inc }}$, which is calculated from REMPI spectra probing the 8-17 band in the incoming beam for dump on and dump off using Equation 4.12. The ratio is found to be 0.45, 0.34, and 1.07 for molecular beams with incidence translational energies of $0.26 \mathrm{eV}, 0.41 \mathrm{eV}$, and $0.57 \mathrm{eV}$, respectively. The scatter of these values indicates the large error of this estimation. In addition, the absence of $v^{\prime \prime}=1$ and 2 in the scattered beam is confirmed by $(2+1)$ REMPI via the B state, supporting the hypothesis that highly vibrationally excited $\mathrm{CO}$ does not relax to low $v^{\prime \prime}$ states when it scatters from $\mathrm{Au}(111)$.

The analysis is different for scattering experiments on $\mathrm{Ag}(111)$, in which $\mathrm{CO}$ relaxes to vibrational states lower than $v^{\prime \prime}=14$. Panel (A) of Figure 4.6 indicates that $v^{\prime \prime}=14$ is significantly populated relative to $v^{\prime \prime}=17$ when $\mathrm{CO}$ is scattered from $\mathrm{Ag}(111)$. Since the vibrational states $v^{\prime \prime} \leq 13$ are not detected, the total number of scattered molecules $N_{\text {sca }}$ cannot be determined as mentioned in Equation 4.13. In order to determine $N_{\text {sca }}$, the silver surface is chlorinated which passivates the surface and suppresses vibrational relaxation. Panel (B) of Figure 4.6 demonstrates the absence of $v^{\prime \prime}=14$ in the beam scattered from the chlorinated silver surface. Relaxation to $v^{\prime \prime}=15$ or 16 is not observed either. As the molecules are scattered vibrationally elastically, $N_{\text {sca }}$ is derived from the 7-17 and the 8-17 band, following Equation 4.12. $R_{v^{\prime \prime}}^{\text {sca }}$ and $P_{\text {relax }}$ are then determined as described for gold. 


\subsection{Rotational State Distributions}

The REMPI spectra of scattered CO show a distinct rotational structure. Each vibrational band consists of a P-, Q- and R-branch. The population of individual rotational states and the mean rotational energy of scattered molecules can be derived from these spectra. This section describes the underlying analysis, in which a $J$-dependent population factor is determined in a fitting procedure such that the experimental spectrum can be accurately reproduced.

Fitting rotational spectra requires the knowledge of precise rotational line positions. In order to assign the observed rotational lines, $\mathrm{A}^{1} \Pi\left(v^{\prime}, J^{\prime}\right) \leftarrow \mathrm{X}^{1} \Sigma^{+}\left(v^{\prime \prime}, J^{\prime \prime}\right)$ transition frequencies are calculated using the computer program PGOPHER [154, 155]. Spectroscopic constants published by Schneider et al. [102] and Simmons et al. [103] are used to calculate term energies of the $\mathrm{X}^{1} \Sigma^{+}$and $\mathrm{A}^{1} \Pi$ state, respectively. However, due to experimental limitations, the constants provided by Simmons are only accurate for rotational excitations up to $J \approx 20$. Exact line positions for transitions probing $J^{\prime \prime}>20$ are calculated from transition frequencies reported by Gerö [156]. Since Gerö made a careful analysis of perturbations in the $\mathrm{A}^{1} \Pi \rightarrow \mathrm{X}^{1} \Sigma^{+}$system, the list is also used to identify perturbed levels in the $\mathrm{A}^{1} \Pi$ state and to recalculate their term energies. Manually replacing transition frequencies from the PGOPHER output with frequencies calculated from Gerö's data provides an accurate linelist for the following analysis.

The 7-17 and 7-16 vibrational bands are good candidates for the rotational analysis of vibrationally elastically and inelastically scattered molecules. $v^{\prime \prime}=16$ and $v^{\prime \prime}=17$ are probed via the $\mathrm{A}^{1} \Pi\left(v^{\prime}=7\right)$ state. Note that in $\mathrm{A}^{1} \Pi\left(v^{\prime}=7\right)$, the rotational states $J^{\prime}=26,29,32,33,35,38,39,42$ and 43 are perturbed [156]. As the mixing of states may strongly affect transition intensities, rotational transitions involving perturbed states are omitted from the rotational analysis. A list of rotational transition wavenumbers of the 7-17 and the 7-16 band, including transitions to perturbed levels, can be found in Appendix A.1. Figure 4.7 shows assigned rotational transitions for both the 7-16 band and the 7-17 band of surface-scattered CO.

The population factors $P_{J^{\prime \prime}}$ of the rotational states $J^{\prime \prime}$ are determined by fitting a Lorentzian line profile to each rotational line in the observed spectrum. A single rotational line can be labeled by $J^{\prime \prime}$ and the respective rotational branch. The Lorentzian line shape is given by

$$
I(\tilde{\nu})=I_{0} \frac{\gamma / 2 \pi}{\left(\tilde{\nu}-\tilde{\nu}_{0}\right)^{2}+(\gamma / 2)^{2}},
$$

where the natural linewidth $\gamma$ is the full width at half maximum, $I$ is the intensity, $\tilde{\nu}$ is the wavenumber, and $\tilde{\nu}_{0}$ is the resonant wavenumber of the transition. 
(A) 7-16 vibrational band

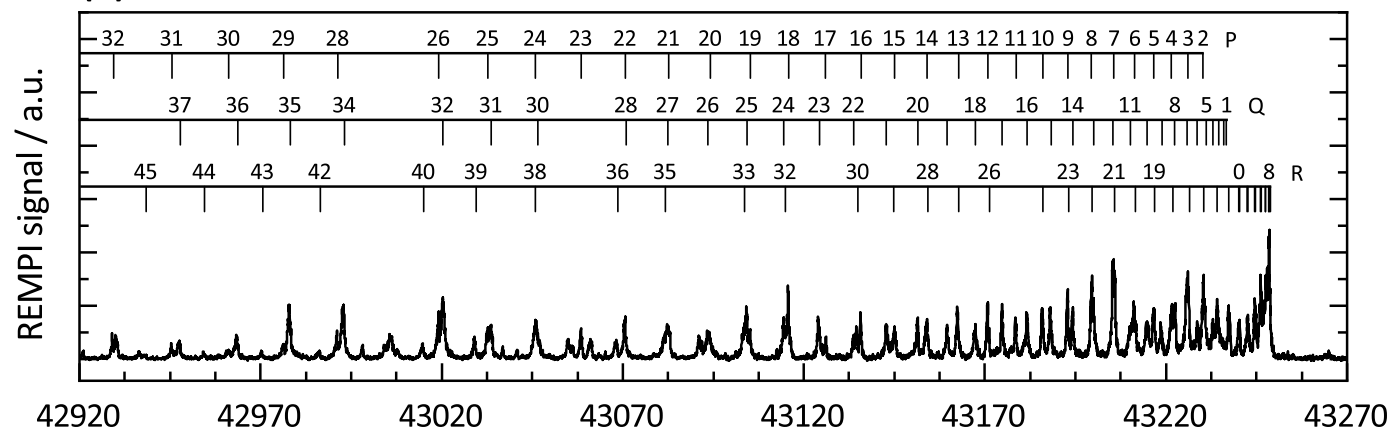

(B) 7-17 vibrational band

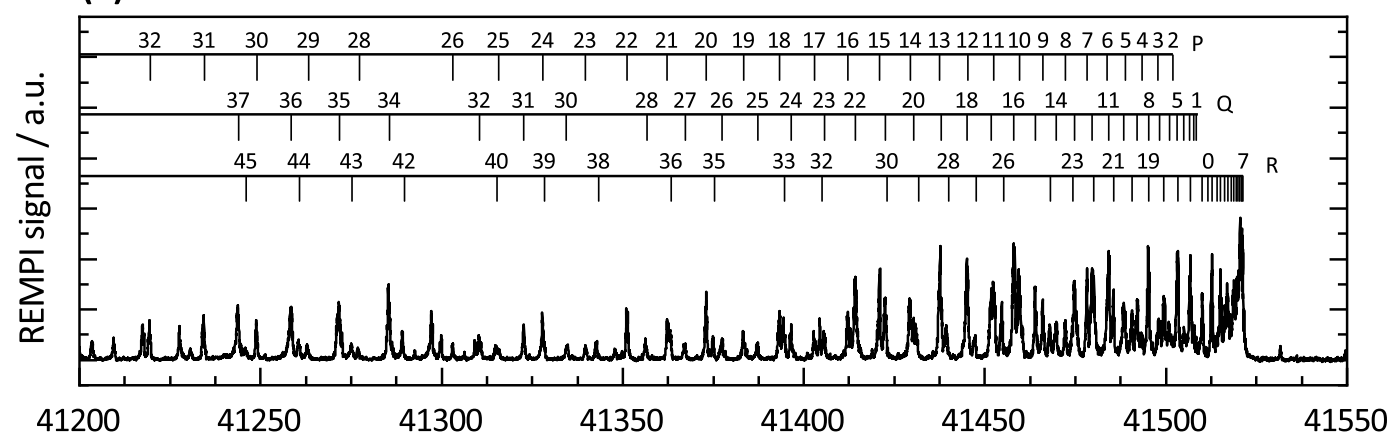

(C) 7-17 vibrational band (zoomed in)

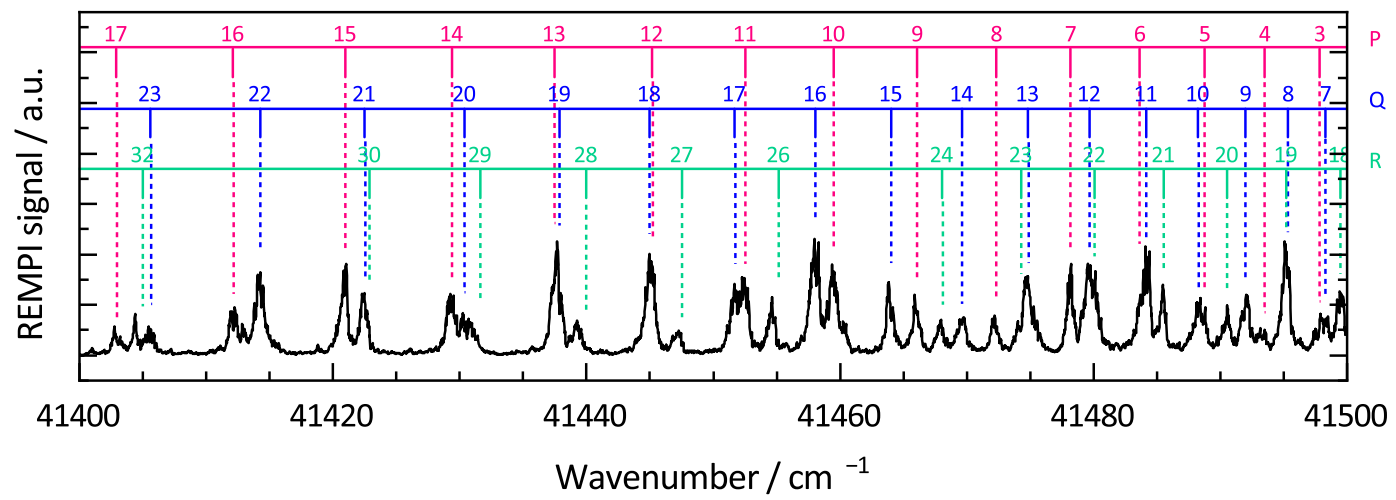

Figure 4.7: $(1+1) \mathrm{A}^{1} \Pi\left(v^{\prime}, J^{\prime}\right) \leftarrow \mathrm{X}^{1} \Sigma^{+}\left(v^{\prime \prime}, J^{\prime \prime}\right)$ REMPI spectra with assigned rotational transitions for $\mathrm{CO}$ scattered from $\mathrm{Au}(111)$ at $\left\langle E_{\text {inc }}\right\rangle=0.57 \mathrm{eV}$. The $\mathrm{R}$-branch exhibits a band head around $\mathrm{R}(7)-\mathrm{R}(8)$. Note that transitions to perturbed states are not marked. Panel (A) shows the 7-16 $\left(v^{\prime}-v^{\prime \prime}\right)$ vibrational band. Panel (B) shows the 7-17 vibrational band. Panel (C) shows an enlargement of the spectrum displayed in Panel (B) in order to illustrate the quality of the assignment. 


\section{Data Analysis}

The total intensity $I_{0}$ of a rovibronic transition is proportional to the population factor $P_{J^{\prime \prime}}$ and to the rotational transition strength $S_{J^{\prime \prime}}^{\text {branch }}$ for a certain $J^{\prime \prime}$ and branch. $S_{J^{\prime \prime}}^{\text {branch }}$ corresponds to the Hönl-London factor as long as the transition is not saturated. In the case of complete saturation, $S_{J^{\prime \prime}}^{\text {branch }}$ equals the number of possible transitions per $J^{\prime \prime}$. In the absence of external fields, each $J^{\prime \prime}$ state is $2 J^{\prime \prime}+1$ degenerate. Thus, the number of possible transitions is $2 J^{\prime \prime}+1$ for the R-branch, $2 J^{\prime \prime}$ for the Q-branch as $M_{J}^{\prime}=0 \leftarrow M_{J}^{\prime \prime}=0$ is forbidden, and $2 J^{\prime \prime}-1$ for the P-branch as transitions from $M_{J}^{\prime \prime}= \pm J^{\prime \prime}$ do not exist. Since the observed REMPI intensity depends linearly on the REMPI laser power (see Figure 4.4), saturation plays a significant role in the resonant excitation step of the two-photon REMPI process. The effect of saturation on the rotational transition strength is treated as described in the PGOPHER manual [154]. In order to account for saturation, $S_{J^{\prime \prime}}^{\text {branch }}$ is expressed in terms of

$$
S_{J^{\prime \prime}}^{\text {branch }}=1-\exp \left[-S_{\text {sat }} \zeta_{J^{\prime \prime}}^{\text {branch }}\right]
$$

where $S_{\text {sat }}$ is the saturation factor and $\zeta_{J^{\prime \prime}}^{\text {branch }}$ is the Hönl-London factor for a certain $J^{\prime \prime}$ and rotational branch. For high $S_{\text {sat }}$, the transition is saturated and $S_{J^{\prime \prime}}^{\text {branch }}$ converges to the saturation limit. If $S_{\text {sat }}$ approaches $0, S_{J^{\prime \prime}}^{\text {branch }}$ equals the product of the HönlLondon factor and the saturation factor, which scales with laser power. Hönl-London factors can be calculated from the rotational matrix elements (see Reference [101] for more details)

$$
\begin{aligned}
& \sum_{\sigma^{\prime}=-1}^{1}\left|\left\langle J^{\prime}, \Lambda^{\prime}, M_{J}^{\prime}\left|D_{\sigma \sigma^{\prime}}^{(\omega) *}\right| J^{\prime \prime}, \Lambda^{\prime \prime}, M_{J}^{\prime \prime}\right\rangle\right|^{2} \\
& \quad=\sum_{\sigma^{\prime}=-1}^{1}\left((-1)^{\Lambda^{\prime}+M_{J}^{\prime}} \sqrt{\left(2 J^{\prime \prime}+1\right)\left(2 J^{\prime}+1\right)}\left(\begin{array}{ccc}
J^{\prime \prime} & \omega & J^{\prime} \\
\Lambda^{\prime \prime} & \sigma^{\prime} & -\Lambda^{\prime}
\end{array}\right)\left(\begin{array}{ccc}
J^{\prime \prime} & \omega & J^{\prime} \\
M_{J}^{\prime \prime} & \sigma & -M_{J}^{\prime}
\end{array}\right)\right)^{2} .
\end{aligned}
$$

Here, $J$ is the total angular momentum, $\Lambda$ is the projection of the electronic orbital angular momentum on the molecular axis, $M_{J}$ is the projection of $J$ in the laboratory frame, $\omega=1$ is the photon angular momentum, $\sigma^{\prime}$ is the polarization of the light in the molecular frame, and $\sigma=0$ is the polarization of the light in the laboratory frame. $\zeta_{J^{\prime \prime}}^{\text {branch }}$ is obtained from Equation 4.18 by summing over $M_{J}^{\prime \prime}$ from $-J^{\prime \prime}+1$ to $J^{\prime \prime}-1$ for the P-branch $\left(\Delta J=J^{\prime}-J^{\prime \prime}=-1\right)$ and from $-J^{\prime \prime}$ to $J^{\prime \prime}$ for the Q-branch $(\Delta J=0)$ and R-branch $(\Delta J=1)$. Note that $\Delta M_{J}=M_{J}^{\prime \prime}-M_{J}^{\prime}=0$.

$S_{\text {sat }}$ is determined in an iterative way by fitting the rotational spectrum for various $S_{\text {sat }}$ and calculating the population factor $P_{J^{\prime \prime}}^{\text {branch }}$ separately for the P-, Q- and Rbranch. The sum of $P_{J^{\prime \prime}}^{\text {branch }}$ over all $J$ should give the same value for each branch. $S_{\text {sat }}$ 
is chosen such that the deviation between summed populations of individual branches is minimized.

Since the $\mathrm{A} \leftarrow \mathrm{X}$ transitions are partially saturated, the observed absorption lines are power broadened. Thus, the natural linewidth $\gamma$ in Equation 4.16 needs to be replaced by a linewidth that accounts for power broadening. According to Equation 4.18, the rotational transition strength decreases with increasing $J^{\prime \prime}$. Following the description by Demtröder [157], the power broadened Lorentzian line width $\gamma_{J^{\prime \prime}}^{\text {branch }}$ is calculated for P-, Q- and R-transitions originating from $J^{\prime \prime}$ as

$$
\gamma_{J^{\prime \prime}}^{\text {branch }}=\Delta \tilde{\nu}_{0} \sqrt{1+S_{\mathrm{sat}} \frac{\zeta_{J^{\prime \prime}}^{\text {branch }}}{g^{\text {branch }}}}
$$

The degeneracy factor $g^{\text {branch }}$ is $2 J^{\prime \prime}-1$ for the P-branch, $2 J^{\prime \prime}$ for the Q-branch, and $2 J^{\prime \prime}+1$ for the R-branch. $\Delta \tilde{\nu}_{0}$ is limited by the spectral width of the frequency doubled dye laser and is estimated to be $0.16 \mathrm{~cm}^{-1}$.

Finally, the rotational population factor $P_{J^{\prime \prime}}$ is determined by fitting the function

$$
I_{J^{\prime \prime}}^{\text {branch }}(\tilde{\nu}) \propto P_{J^{\prime \prime}} S_{J^{\prime \prime}}^{\text {branch }} \frac{\gamma_{J^{\prime \prime}}^{\text {branch }} / 2 \pi}{\left(\tilde{\nu}-\tilde{\nu}_{J^{\prime \prime}}^{\text {branch }}\right)^{2}+\left(\gamma_{J^{\prime \prime}}^{\text {branch }} / 2\right)^{2}}
$$

to each rotational line observed in the REMPI spectrum. The only fit parameter $P_{J^{\prime \prime}}$ is restrained to be the same for all rotational branches and varies only with $J^{\prime \prime}$. Line positions $\tilde{\nu}_{J^{\prime \prime}}^{\text {branch }}$ are listed in Appendix A.1.

A Boltzmann plot of rotational states $J^{\prime \prime}$ is obtained by plotting $\ln \left(P_{J^{\prime \prime}}\right)$ as a function of the rotational energy of the scattered molecule. Note that $P_{J^{\prime \prime}}$ need not to be divided by the degeneracy of rotational states due to the definition of $S_{J^{\prime \prime}}^{\text {branch }}$. The rotational energy of a molecule in the highly vibrationally excited $\mathrm{X}^{1} \Sigma^{+}\left(v^{\prime \prime}\right)$ state is given by

$$
E\left(v^{\prime \prime}, J^{\prime \prime}\right)-E\left(v^{\prime \prime}, J^{\prime \prime}=0\right)
$$

where $E\left(v^{\prime \prime}, J^{\prime \prime}\right)$ can be expressed in terms of the standard Dunham expansion [101]

$$
E\left(v^{\prime \prime}, J^{\prime \prime}\right)=\sum_{k=0}^{\infty} \sum_{l=0}^{\infty} Y_{k l}\left(v^{\prime \prime}+\frac{1}{2}\right)^{k}\left[J^{\prime \prime}\left(J^{\prime \prime}+1\right)-\Lambda^{\prime \prime 2}\right]^{l}
$$

For $\operatorname{CO~X}{ }^{1} \Sigma^{+}, \Lambda^{\prime \prime}=0 . Y_{k l}$ are the Dunham expansion coefficients, which are listed for $\mathrm{CO} \mathrm{X}^{1} \Sigma^{+}$in Table 1 of Reference [102].

The mean rotational energy of scattered molecules is calculated as

$$
\left\langle E_{\text {rot }}\left(v^{\prime \prime}\right)\right\rangle=\sum_{J^{\prime \prime}=0}^{\infty} \frac{\left(2 J^{\prime \prime}+1\right) P_{J^{\prime \prime}}}{\sum_{J^{\prime \prime}=0}^{\infty}\left(2 J^{\prime \prime}+1\right) P_{J^{\prime \prime}}}\left[E\left(v^{\prime \prime}, J^{\prime \prime}\right)-E\left(v^{\prime \prime}, J^{\prime \prime}=0\right)\right] .
$$





\section{Surface Scattering of $\mathrm{CO} \mathrm{X}^{1} \Sigma^{+}(v=17)$}

This chapter presents and discusses the results obtained from state-to-state surface scattering experiments using molecular beams of highly vibrationally excited CO. The experimental data comprises time-of-flight distributions, angular distributions, and REMPI spectra of surface scattered CO, which are used to extract final vibrational state and rotational state distributions. Final vibrational state distributions of highly vibrationally excited $\mathrm{CO}$ scattered from $\mathrm{Au}(111)$ are published in Reference [31]. For the scattering from $\operatorname{Ag}(111)$, final vibrational state distributions and angular distributions are published in Reference [32].

\subsection{Time-of-Flight Distributions}

\subsubsection{Results}

The mean translational energy $\left\langle E_{\mathrm{sca}}\right\rangle$ of surface scattered molecules can be derived from time-of-flight distributions, as described in Section 4.1. State-to-state time-of-flight distributions have been measured for different final vibrational $\left(v_{\text {sca }}\right)$ and rotational $\left(J_{\text {sca }}\right)$ states. Single rovibrational states are probed during the time-of-flight scan by setting the probe laser to the frequency of a resolved rovibronic transition. Since the initial energetic state of incident $\mathrm{CO}$ is well defined by $\left\langle E_{\text {inc }}\right\rangle, v_{\text {inc }}=17$, and $J_{\text {inc }}=0$, the information extracted from time-of-flight distributions of scattered molecules allow the investigation of translation-to-rotation coupling (T-R) and vibration-to-translation coupling (V-T) during the surface scattering event.

The upper panels of Figure 5.1(a) show time-of-flight distributions of CO molecules that scatter vibrationally elastically from $\mathrm{Au}(111)$. The distributions have been measured for three different incidence translational energies (left, center, right). Each plot contains the data obtained for three scattering products, $J_{\text {sca }}=6,14$, and 27 (black, red, blue). The arrival time on the horizontal axis corresponds to the flight time of scattered molecules from the surface to the point of detection. The point of detection is located $4.3 \mathrm{~mm}$ in front of the surface and is chosen such that the scattered beam is probed at the maximum of the angular distribution. The solid lines represent the best fit to the time-of-flight data using Equation 4.6. The corresponding velocity distributions 




Arrival time / $\mu \mathrm{s}$

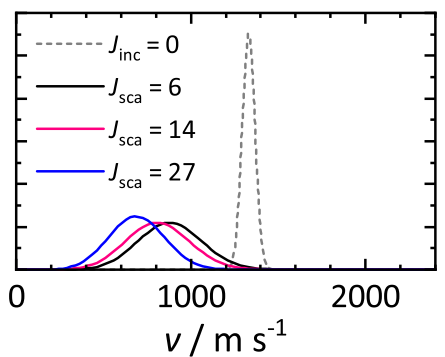

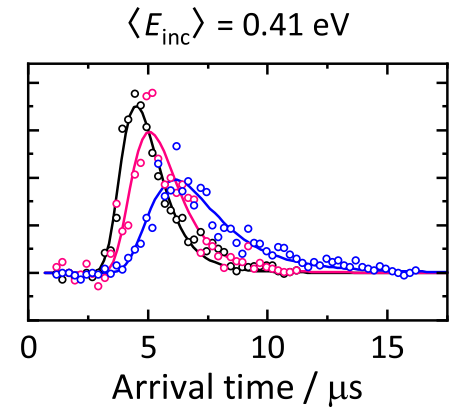

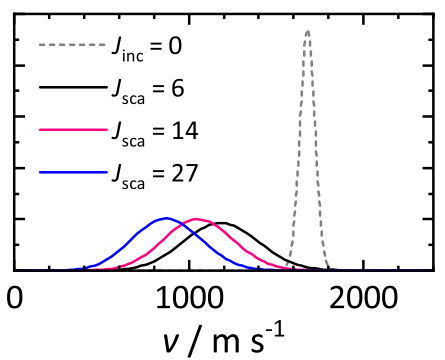

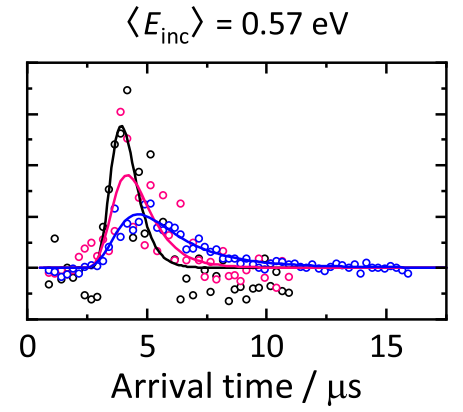

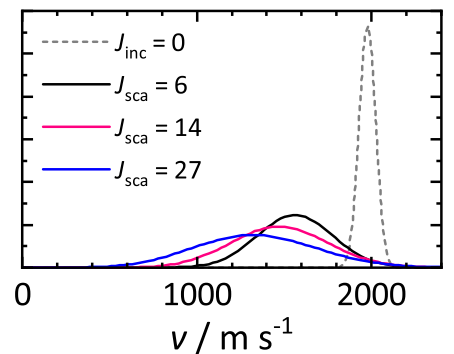

(a) $v_{\text {inc }}=17 \rightarrow v_{\text {sca }}=17$ scattering of $\mathrm{CO}$ on $\mathrm{Au}(111)$.
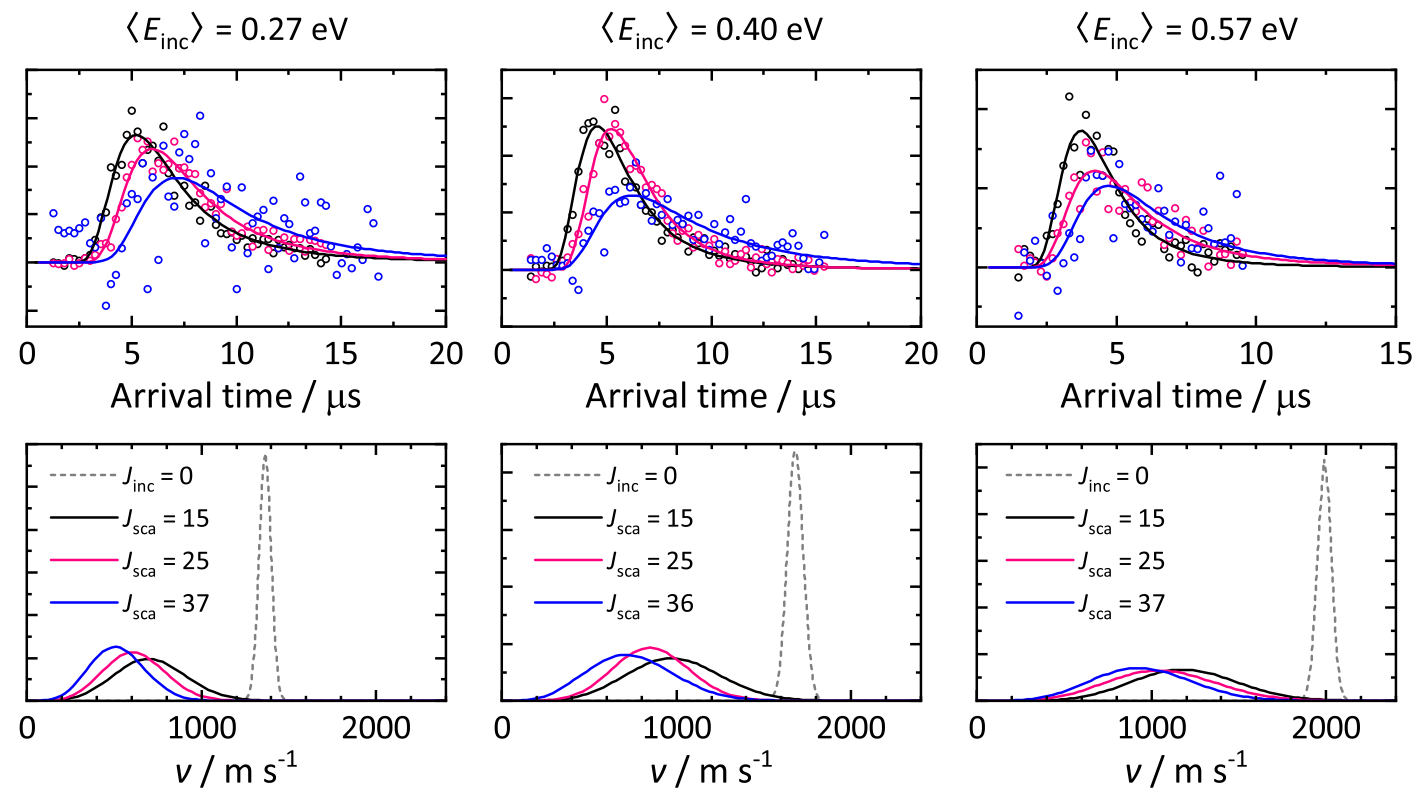

(b) $v_{\text {inc }}=17 \rightarrow v_{\text {sca }}=17$ scattering of $\mathrm{CO}$ on $\mathrm{Ag}(111)$.

Figure 5.1: Normalized arrival time distributions (upper panels) and corresponding velocity distributions (lower panels) are shown for three incidence translational energies (columns) and three final rotational states (see color code). The velocity distribution of the incident beam is also shown in the lower panels (gray dashed). 
are shown in the lower panels of Figure 5.1(a). Tables 5.1 summarizes the final translational energies and velocities as well as the fitting parameters for the scattering from $\mathrm{Au}(111)$. Results for the scattering from $\mathrm{Ag}(111)$ are given in Figure 5.1(b) and Table 5.2. Note that the detected rotational states differ from those probed in experiments with gold.

Table 5.1: Fitting parameters $N_{v}, v_{0}$, and $w_{v}$ from Equation 4.6, final translational energies $\left\langle E_{\text {sca }}\right\rangle$, and mean velocities $\left\langle v_{\text {sca }}\right\rangle$ of $\operatorname{CO}\left(v_{\text {inc }}=17, J_{\text {inc }}=0\right)$ scattered from $\mathrm{Au}(111)$ are given for different incidence translational energies $\left\langle E_{\text {inc }}\right\rangle$ and final scattering products $\left(v_{\text {sca }}, J_{\text {sca }}\right)$.

\begin{tabular}{clccccc}
\hline \hline $\begin{array}{c}\left\langle\boldsymbol{E}_{\text {inc }}\right\rangle / \\
\mathbf{e V}\end{array}$ & Scattering & $\left\langle\begin{array}{c}\left\langle\boldsymbol{E}_{\text {sca }}\right\rangle / \\
\mathbf{e V}\end{array}\right.$ & $\begin{array}{c}\left\langle\boldsymbol{v}_{\text {sca }}\right\rangle / \\
\mathbf{m ~ s}^{-\mathbf{1}}\end{array}$ & $\begin{array}{c}\boldsymbol{N}_{\boldsymbol{v}} / \\
\mathbf{m}^{-\mathbf{3}}\end{array}$ & $\begin{array}{c}\boldsymbol{v}_{\mathbf{0}} / \\
\mathbf{m} \mathbf{~ s}^{-\mathbf{1}}\end{array}$ & $\begin{array}{c}\boldsymbol{w}_{\boldsymbol{v}} / \\
\mathbf{m ~ s}^{-\mathbf{1}}\end{array}$ \\
\hline 0.57 & $v_{\text {sca }}=17, J_{\text {sca }}=6$ & 0.36 & 1564 & $1.88 \cdot 10^{12}$ & 1466 & 315 \\
0.57 & $v_{\text {sca }}=17, J_{\text {sca }}=14$ & 0.33 & 1477 & $1.85 \cdot 10^{12}$ & 1294 & 417 \\
0.57 & $v_{\text {sca }}=17, J_{\text {sca }}=27$ & 0.28 & 1343 & $1.34 \cdot 10^{12}$ & 964 & 561 \\
0.41 & $v_{\text {sca }}=17, J_{\text {sca }}=6$ & 0.21 & 1186 & $7.51 \cdot 10^{11}$ & 1051 & 320 \\
0.41 & $v_{\text {sca }}=17, J_{\text {sca }}=14$ & 0.17 & 1056 & $4.84 \cdot 10^{11}$ & 924 & 300 \\
0.41 & $v_{\text {sca }}=17, J_{\text {sca }}=27$ & 0.12 & 876 & $2.45 \cdot 10^{11}$ & 709 & 304 \\
0.26 & $v_{\text {sca }}=17, J_{\text {sca }}=6$ & 0.12 & 883 & $2.46 \cdot 10^{11}$ & 747 & 276 \\
0.26 & $v_{\text {sca }}=17, J_{\text {sca }}=14$ & 0.10 & 813 & $1.81 \cdot 10^{11}$ & 659 & 281 \\
0.26 & $v_{\text {sca }}=17, J_{\text {sca }}=27$ & 0.07 & 692 & $9.60 \cdot 10^{10}$ & 548 & 250 \\
\hline \hline
\end{tabular}

Table 5.2: Fitting parameters $N_{v}, v_{0}$, and $w_{v}$ from Equation 4.6, final translational energies $\left\langle E_{\mathrm{sca}}\right\rangle$, and mean velocities $\left\langle v_{\mathrm{sca}}\right\rangle$ of $\mathrm{CO}\left(v_{\mathrm{inc}}=17, J_{\mathrm{inc}}=0\right)$ scattered from $\mathrm{Ag}(111)$ are given for different incidence translational energies $\left\langle E_{\text {inc }}\right\rangle$ and final scattering products $\left(v_{\text {sca }}, J_{\text {sca }}\right)$. Note that for vibrationally elastic scattering, the REMPI signal is integrated over the band head of the $\mathrm{R}$ branch $\left(5 \leq J_{\text {sca }} \leq 8\right)$.

\begin{tabular}{|c|c|c|c|c|c|c|}
\hline $\begin{array}{c}\left\langle E_{\text {inc }}\right\rangle / \\
\mathrm{eV}\end{array}$ & $\begin{array}{l}\text { Scattering } \\
\text { product }\end{array}$ & $\begin{array}{c}\left\langle\boldsymbol{E}_{\mathrm{sca}}\right\rangle / \\
\mathrm{eV}\end{array}$ & $\begin{array}{c}\left\langle v_{\text {sca }}\right\rangle / \\
\mathbf{m s}^{-1}\end{array}$ & $\begin{array}{l}N_{v} / \\
\mathbf{m}^{-3}\end{array}$ & $\begin{array}{c}v_{0} / \\
\mathrm{ms}^{-1}\end{array}$ & $\begin{array}{c}w_{v} / \\
\mathbf{m s}^{-1}\end{array}$ \\
\hline \multicolumn{7}{|c|}{ Vibrationally inelastic scattering: } \\
\hline 0.57 & $v_{\mathrm{sca}}=17, J_{\mathrm{sca}}=7$ & 0.21 & 1165 & $1.30 \cdot 10^{-12}$ & 915 & 428 \\
\hline 0.57 & $v_{\mathrm{sca}}=17, J_{\mathrm{sca}}=15$ & 0.22 & 1197 & $1.17 \cdot 10^{-12}$ & 894 & 474 \\
\hline 0.57 & $v_{\mathrm{sca}}=17, J_{\mathrm{sca}}=25$ & 0.18 & 1068 & $2.00 \cdot 10^{-12}$ & 658 & 514 \\
\hline
\end{tabular}


5 Surface Scattering of $C O X^{1} \Sigma^{+}(v=17)$

Table 5.2: (continued)

\begin{tabular}{|c|c|c|c|c|c|c|}
\hline $\begin{array}{c}\left\langle\boldsymbol{E}_{\text {inc }}\right\rangle / \\
\mathrm{eV}\end{array}$ & $\begin{array}{l}\text { Scattering } \\
\text { product }\end{array}$ & $\begin{array}{c}\left\langle\boldsymbol{E}_{\mathrm{sca}}\right\rangle / \\
\mathrm{eV}\end{array}$ & $\begin{array}{c}\left\langle v_{\text {sca }}\right\rangle / \\
\mathrm{m} \mathrm{s}^{-1}\end{array}$ & $\begin{array}{l}N_{v} / \\
\mathbf{m}^{-3}\end{array}$ & $\begin{array}{c}v_{0} / \\
\mathrm{ms}^{-1}\end{array}$ & $\begin{array}{c}w_{v} / \\
\mathrm{ms}^{-1}\end{array}$ \\
\hline 0.57 & $v_{\mathrm{sca}}=17, J_{\mathrm{sca}}=31$ & 0.13 & 912 & $3.55 \cdot 10^{-12}$ & 628 & 399 \\
\hline 0.57 & $v_{\mathrm{sca}}=17, J_{\mathrm{sca}}=37$ & 0.14 & 955 & $3.26 \cdot 10^{-12}$ & 548 & 484 \\
\hline 0.40 & $v_{\mathrm{sca}}=17, J_{\mathrm{sca}}=7$ & 0.17 & 1058 & $1.92 \cdot 10^{-12}$ & 774 & 431 \\
\hline 0.40 & $v_{\mathrm{sca}}=17, J_{\mathrm{sca}}=15$ & 0.15 & 991 & $2.56 \cdot 10^{-12}$ & 679 & 436 \\
\hline 0.40 & $v_{\mathrm{sca}}=17, J_{\mathrm{sca}}=25$ & 0.11 & 859 & $4.41 \cdot 10^{-12}$ & 642 & 341 \\
\hline 0.40 & $v_{\mathrm{sca}}=17, J_{\mathrm{sca}}=31$ & 0.12 & 865 & $5.00 \cdot 10^{-12}$ & 474 & 450 \\
\hline 0.40 & $v_{\mathrm{sca}}=17, J_{\mathrm{sca}}=36$ & 0.09 & 748 & $1.12 \cdot 10^{-11}$ & 275 & 455 \\
\hline 0.27 & $v_{\mathrm{sca}}=17, J_{\mathrm{sca}}=7$ & 0.07 & 676 & $1.21 \cdot 10^{-11}$ & 445 & 308 \\
\hline 0.27 & $v_{\mathrm{sca}}=17, J_{\mathrm{sca}}=15$ & 0.08 & 713 & $1.01 \cdot 10^{-11}$ & 442 & 342 \\
\hline 0.27 & $v_{\mathrm{sca}}=17, J_{\mathrm{sca}}=25$ & 0.06 & 620 & $1.74 \cdot 10^{-11}$ & 393 & 292 \\
\hline 0.27 & $v_{\mathrm{sca}}=17, J_{\mathrm{sca}}=31$ & 0.05 & 589 & $2.45 \cdot 10^{-11}$ & 297 & 320 \\
\hline 0.27 & $v_{\mathrm{sca}}=17, J_{\mathrm{sca}}=37$ & 0.04 & 522 & $3.77 \cdot 10^{-11}$ & 284 & 273 \\
\hline \multicolumn{7}{|c|}{ Vibrationally elastic scattering: } \\
\hline 0.57 & $v_{\mathrm{sca}}=17,5 \leq J_{\mathrm{sca}} \leq 8$ & 0.22 & 1199 & $1.17 \cdot 10^{-12}$ & 978 & 410 \\
\hline 0.57 & $v_{\mathrm{sca}}=16,5 \leq J_{\mathrm{sca}} \leq 8$ & 0.21 & 1159 & $1.37 \cdot 10^{-12}$ & 789 & 513 \\
\hline 0.57 & $v_{\mathrm{sca}}=15,5 \leq J_{\mathrm{sca}} \leq 8$ & 0.26 & 1297 & $9.15 \cdot 10^{-13}$ & 810 & 619 \\
\hline 0.57 & $v_{\mathrm{sca}}=14,5 \leq J_{\mathrm{sca}} \leq 8$ & 0.26 & 1303 & $8.76 \cdot 10^{-13}$ & 853 & 597 \\
\hline 0.40 & $v_{\mathrm{sca}}=17,5 \leq J_{\mathrm{sca}} \leq 8$ & 0.15 & 993 & $2.48 \cdot 10^{-12}$ & 721 & 409 \\
\hline 0.40 & $v_{\mathrm{sca}}=16,5 \leq J_{\mathrm{sca}} \leq 8$ & 0.15 & 991 & $2.50 \cdot 10^{-12}$ & 737 & 395 \\
\hline 0.40 & $v_{\mathrm{sca}}=15,5 \leq J_{\mathrm{sca}} \leq 8$ & 0.20 & 1135 & $1.47 \cdot 10^{-12}$ & 795 & 488 \\
\hline 0.40 & $v_{\mathrm{sca}}=14,5 \leq J_{\mathrm{sca}} \leq 8$ & 0.21 & 1167 & $1.36 \cdot 10^{-12}$ & 762 & 536 \\
\hline 0.30 & $v_{\mathrm{sca}}=17,5 \leq J_{\mathrm{sca}} \leq 8$ & 0.11 & 834 & $6.27 \cdot 10^{-12}$ & 398 & 464 \\
\hline 0.30 & $v_{\mathrm{sca}}=16,5 \leq J_{\mathrm{sca}} \leq 8$ & 0.11 & 825 & $5.28 \cdot 10^{-12}$ & 576 & 356 \\
\hline 0.30 & $v_{\mathrm{sca}}=15,5 \leq J_{\mathrm{sca}} \leq 8$ & 0.13 & 922 & $3.37 \cdot 10^{-12}$ & 653 & 391 \\
\hline 0.30 & $v_{\mathrm{sca}}=14,5 \leq J_{\mathrm{sca}} \leq 8$ & 0.12 & 883 & $4.29 \cdot 10^{-12}$ & 543 & 427 \\
\hline 0.27 & $v_{\mathrm{sca}}=17,5 \leq J_{\mathrm{sca}} \leq 8$ & 0.08 & 695 & $1.12 \cdot 10^{-11}$ & 426 & 336 \\
\hline 0.27 & $v_{\mathrm{sca}}=16,5 \leq J_{\mathrm{sca}} \leq 8$ & 0.08 & 735 & $8.55 \cdot 10^{-12}$ & 493 & 330 \\
\hline 0.27 & $v_{\mathrm{sca}}=15,5 \leq J_{\mathrm{sca}} \leq 8$ & 0.08 & 727 & $8.57 \cdot 10^{-12}$ & 548 & 284 \\
\hline 0.27 & $v_{\mathrm{sca}}=14,5 \leq J_{\mathrm{sca}} \leq 8$ & 0.08 & 703 & $1.73 \cdot 10^{-11}$ & 172 & 464 \\
\hline
\end{tabular}

The reader may notice that the time-of-flight traces are measured fairly close to the surface. In order to minimize the relative error of the travel distance, the time-of-flight traces should be measured as far away from the surface as possible. However, the travel 
distance is limited to $4.3 \mathrm{~mm}$ because the signal is too weak at larger distances as it gets diluted due to the temporal and angular spread of the beam. The detection is challenging because of the small number of highly vibrationally excited $\mathrm{CO}$ molecules in the incident beam. The small number of molecules results from the inefficient preparation of highly vibrationally excited $\mathrm{CO}$, which involves three laser-driven optical transitions including weak transitions between the singlet and the triplet manifolds. Furthermore, the detection is challenging because high $v$ states other than $v=17$ are populated by means of Franck-Condon pumping which results in an undesired background signal. Figure 5.1 shows the background corrected data. In contrast to $\mathrm{CO}$, the preparation of high $v$ states is less demanding for NO because the optical pumping scheme involves only two spin-allowed transitions and Franck-Condon pumping can be suppressed by a process called sweeping [158], resulting in a much better signal-to-noise ratio.

Two conclusions can be drawn from the velocity distributions shown in Figure 5.1. First, vibrationally elastically scattered molecules lose translational energy during the collision. Second, the translational energy loss increases with rotational excitation of the scattered molecules.

The correlation between final translational energy $\left\langle E_{\text {sca }}\right\rangle$ and final rotational energy $E_{\text {rot }}$ is illustrated in Figure 5.2. The error bars are based on the error in the measurement of the flight distance, which is the largest source of uncertainty in this experiment. In order to determine the probe laser position, the surface is moved into the power-reduced laser beam, whose profile is monitored on a screen. The surface is assumed to be at the probe laser position when the laser spot is half eclipsed by the surface. The surface is mounted on a micrometer with $10 \mu \mathrm{m}$ resolution. Following this procedure, the position of laser focus can be determined with an accuracy of $\Delta x=200 \mu \mathrm{m}$. Error propagation yields the error bars shown for $\left\langle E_{\text {sca }}\right\rangle$ in Figure 5.2.

Time-of-flight traces have been also determined for molecules that undergo vibrationally inelastic scattering and lose vibrational energy during the surface collision. Inelastic scattering results are only available from $\mathrm{Ag}(111)$. The final vibrational states $v_{\text {sca }}=14,15,16$ and 17 are detected by probing the rotational band head of the Rbranch, which exhibits a strong signal but consists of multiple overlapping transitions ranging from $R(5)$ to $R(8)$. This means that the detected molecules experience a small amount of rotational excitation. The measurement of multiple rotational lines and extrapolation to $J_{\text {sca }}=0$ is not feasible due to the weak signal of vibrationally relaxed molecules. Figure 5.3 illustrates the vibration-to-translation (V-T) coupling by plotting the final translational energy as a function of the final vibrational energy. For $\left\langle E_{\text {inc }}\right\rangle=0.57 \mathrm{eV}$, the final translational energy clearly increases with vibrational energy loss. However, this effect depends on $\left\langle E_{\text {inc }}\right\rangle$ and almost vanishes for low $\left\langle E_{\text {inc }}\right\rangle$ as shown 
(A) $\mathrm{Au}(111)$

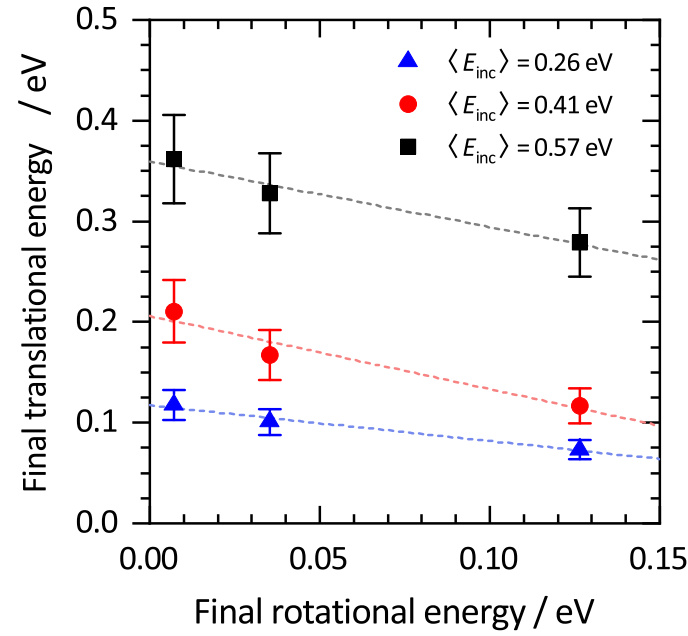

(B) $\operatorname{Ag}(111)$

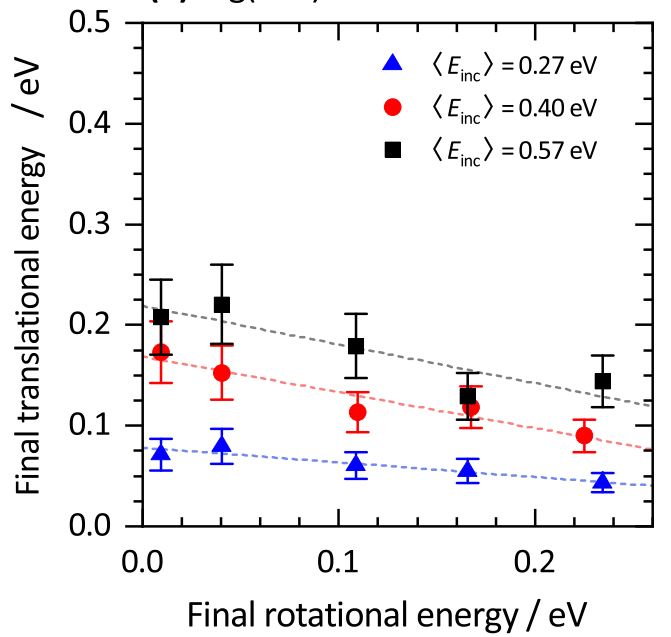

Figure 5.2: Final translational energy versus final rotational energy for vibrationally elastically scattered $\mathrm{CO}\left(v_{\text {inc }}=17, J_{\text {inc }}=0\right)$. Panel $(\mathrm{A})$ shows results obtained for $\mathrm{Au}(111)$, where rotational states between $J_{\text {sca }}=6$ and 27 have been detected. Panel (B) shows results obtained for $\operatorname{Ag}(111)$, where rotational states between $J_{\text {sca }}=7$ and 37 have been detected. The dashed lines represent linear fits to the data. Note that the final translational energy of molecules scattered from silver is in general lower than that of molecules scattered from gold because of the lower atomic mass of silver.

for $0.27 \mathrm{eV}$.

\subsubsection{Discussion: Translational Inelasticity}

Vibrationally and Rotationally Elastic Scattering The velocity distributions in Figure 5.1 indicate that the scattered molecules lose a large fraction of their initial translational energy during the collision with the surface. For incidence translational energies in the range of $0.26 \mathrm{eV} \leq\left\langle E_{\text {inc }}\right\rangle \leq 0.57 \mathrm{eV}$, final translational energies are found between $0.07 \mathrm{eV}$ and $0.36 \mathrm{eV}$ for gold, and between $0.04 \mathrm{eV}$ and $0.22 \mathrm{eV}$ for silver. What is the fate of the molecule's translational energy? Different energy dissipation pathways are conceivable for the molecule-surface system. The translational energy may either excite surface degrees of freedom or internal degrees of freedom of the molecule, such as vibration and rotation. In the case of vibrationally and rotationally elastic scattering, where $\left(v_{\text {inc }}=17, J_{\text {inc }}=0\right) \rightarrow\left(v_{\text {sca }}=17, J_{\text {sca }}=0\right)$, excitation of molecular vibration 


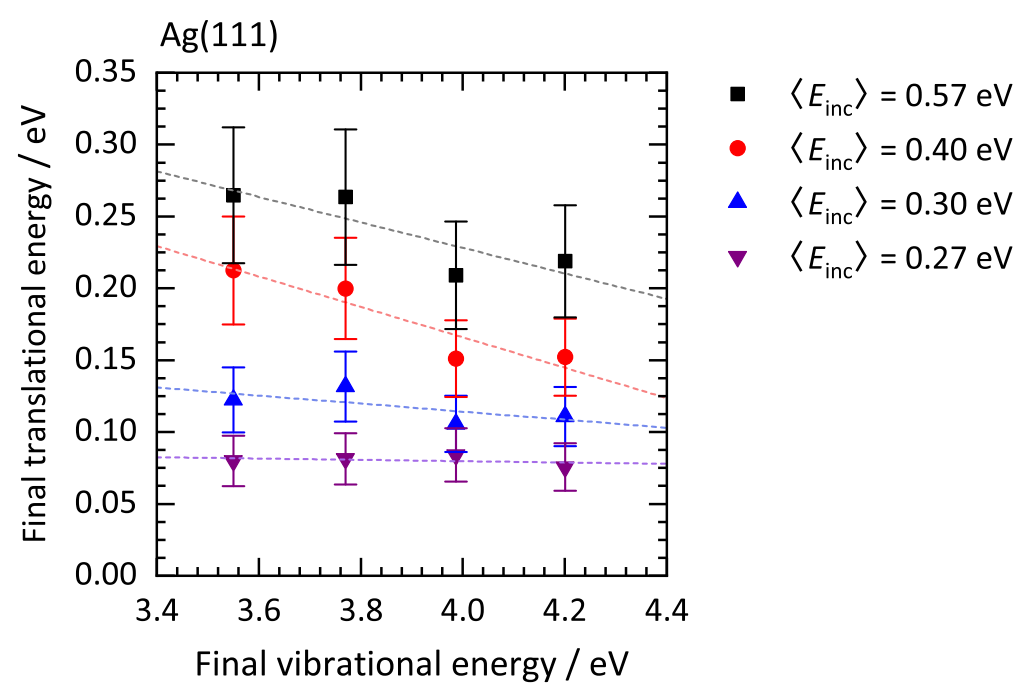

Figure 5.3: Final translational energy versus final vibrational energy for $\mathrm{CO}\left(v_{\text {inc }}=17\right.$, $\left.J_{\text {inc }}=0\right)$ scattered from $\mathrm{Ag}(111)$. Results are shown for four incidence translational energies. The final vibrational states $v_{\text {sca }}=14,15,16$ and 17 have been detected by probing the rotational band heads of the respective R-branches. The dashed lines show linear fits to the experimental data.

and rotation can be excluded. Thus, the fraction of translational energy lost during the surface collision is completely transferred to the surface.

The dependence of the final translational energy $\left\langle E_{\text {sca }}\right\rangle$ on the incidence translational energy $\left\langle E_{\text {inc }}\right\rangle$ is illustrated in Figure 5.4. $\left\langle E_{\text {sca }}\right\rangle$ of vibrationally and rotationally elastically scattered $\mathrm{CO}$ is derived by extrapolating the data shown in Figure 5.2, using the linear fits. Figure 5.4 shows two trends. First, the translational inelasticity is stronger for silver than for gold. Second, $\left\langle E_{\text {sca }}\right\rangle$ seems to increase linearly with $\left\langle E_{\text {inc }}\right\rangle$. The molecules retain $\approx 40 \%$ and $\approx 50 \%$ of their incidence translational energy when scattered from silver and gold, respectively.

The transfer of translational energy to the surface is assumed to follow an electronically adiabatic pathway. In order to test this hypothesis, the experimental results are compared to $\left\langle E_{\text {sca }}\right\rangle$ predicted by the hard-cube model [159]. The hard-cube model is a simple model which assumes a purely mechanical energy transfer. In this model, the CO molecule is represented by a hard sphere with mass $m_{1}=28.0 \mathrm{amu}$ which impinges on the surface of a hard cube with the mass $m_{2}$. Based on the conservation laws of energy and linear momentum, the hard-cube model predicts $\left\langle E_{\text {sca }}\right\rangle$ for an incident trajectory 


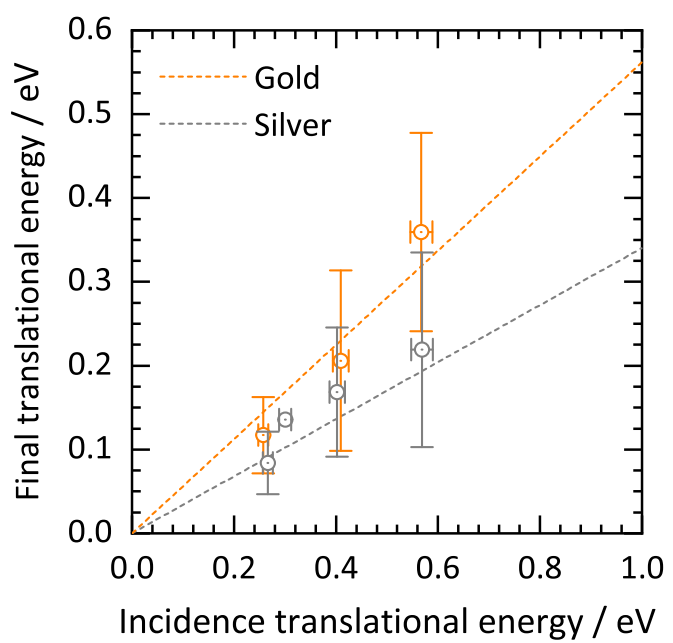

Figure 5.4: Extrapolated final translational energy of $\mathrm{CO}\left(v_{\mathrm{sca}}=17, J_{\mathrm{sca}}=0\right)$ versus incidence translational energy of $\mathrm{CO}\left(v_{\text {inc }}=17, J_{\text {inc }}=0\right)$ for the scattering from $\mathrm{Au}(111)$ (orange) and $\mathrm{Ag}(111)$ (gray). The dashed lines show the final translational energy predicted by the Baule model for collision with a single metal atom.

parallel to the surface normal as

$$
\left\langle E_{\text {sca }}\right\rangle=\frac{\left(m_{1}-m_{2}\right)^{2}}{\left(m_{1}+m_{2}\right)^{2}}\left\langle E_{\text {inc }}\right\rangle
$$

Two limiting cases are conceivable for $\left\langle E_{\text {sca }}\right\rangle$. First, the amount of energy transferred to the surface is negligible when $m_{2}$ is considerably larger than $m_{1}$. This scenario corresponds to a stiff surface with immobile surface atoms. Second, the upper limit of translational inelasticity - commonly known as the Baule limit - can be estimated by assuming the collision with a single metal atom as collision partner $\left(m_{2}=m_{\mathrm{Au}}=\right.$ $197.0 \mathrm{amu}$ or $m_{2}=m_{\mathrm{Ag}}=107.9 \mathrm{amu}$ ) [160]. According to the Baule limit, $34 \%$ and $56 \%$ of $\left\langle E_{\text {inc }}\right\rangle$ is preserved in collisions with silver and gold, respectively. These values are remarkably close to the ones found in the experiment and confirm that the momentum transfer is more efficient for silver than for gold due to the lower atomic mass of silver.

In Figure 5.4, the Baule limit is indicated by the dashed lines. Note that in the hard-cube model, only momentum parallel to the surface normal can be transferred to surface atoms, while the momentum perpendicular to the surface normal is conserved. Accordingly, the Baule limits shown in Figure 5.4 account for the slight tilt of the 
molecular beam axis with respect to the surface normal.

$$
\left\langle E_{\text {sca }}\right\rangle=\frac{\left(m_{1}-m_{2}\right)^{2}}{\left(m_{1}+m_{2}\right)^{2}}\left\langle E_{\text {inc }}\right\rangle \cos ^{2}\left(\theta_{\text {inc }}\right)+\left\langle E_{\text {inc }}\right\rangle\left(1-\cos ^{2}\left(\theta_{\text {inc }}\right)\right)
$$

The incidence angle $\theta_{\text {inc }}$ is $3^{\circ}$ for experiments with gold and $5^{\circ}$ for experiments with silver. Within the error bars, the slope of the Baule limit agrees with the observed incidence translational energy dependence. The fact that $\left\langle E_{\text {sca }}\right\rangle$ depends linearly on $\left\langle E_{\text {inc }}\right\rangle$ can be interpreted in terms of a direct scattering mechanism. A characteristic feature of direct scattering is the preservation of information about the molecule's initial properties such as velocity and incidence angle. In contrast, the molecules would lose their "memory" of incidence velocity and angle when undergoing a trapping-desorption (TD) scattering mechanism. In the TD mechanism, the molecules equilibrate thermally with the surface during the residence time on the surface. The velocity distribution of the desorbing molecules can then be described by the Maxwell-Boltzmann flux distribution, treating the surface as an effusive beam source. The mean translational energy does not depend on $\left\langle E_{\text {inc }}\right\rangle$. Rather, it is given by $2 k_{\mathrm{B}} T_{\mathrm{s}}$, where $T_{\mathrm{s}}$ is the surface temperature. Note that this estimation does not account for energy barriers the desorbing molecules may have to surmount. Based on these assumptions for the trapping-desorption mechanism, the final translational energy of desorbing molecules is expected to be $0.052 \mathrm{eV}$ and $0.068 \mathrm{eV}$ for experiments with gold $\left(T_{\mathrm{s}}=300 \mathrm{~K}\right)$ and silver $\left(T_{\mathrm{s}}=400 \mathrm{~K}\right)$, respectively. This is in stark contrast to the experimental findings, which clearly indicate a direct scattering channel.

The comparison of the Baule limit with experimental results has been previously reported for different molecule-surface systems. Krüger investigated the translational inelasticity of highly vibrationally excited NO scattered from gold [129]. For the $\mathrm{NO}\left(v_{\text {inc }}=\right.$ 11) $/ \mathrm{Au}(111)$ system, the Baule limit is in good agreement with experimentally determined $\left\langle E_{\text {sca }}\right\rangle$ of vibrationally elastically scattered NO. Golibrzuch et al. presented results on the $\mathrm{NO}\left(v_{\text {inc }}=3\right) / \mathrm{Au}(111)$ system $[80,81]$. State-to-state time-of-flight measurements with high resolutions reveal that the Baule limit slightly overestimates the translational energy loss. This agrees with results published by Rettner et al. who studied the $\mathrm{NO}\left(v_{\text {inc }}=0\right) / \mathrm{Ag}(111)$ system for an incidence angle of $\theta_{\text {inc }}=30^{\circ}$ [161] . Here, the Baule limit predicts a translational energy loss of $59 \%$, which again exceeds the experimental value. The comparison of the results by Golibrzuch et al. and Rettner et al. confirm that the translational inelasticity is stronger for silver than for gold.

To sum up, the translational energy loss of highly vibrationally excited CO in vibrationally and rotationally elastic surface scattering is in good agreement with predictions based on the simple Baule model. Even though the physical meaning of the Baule limit should not be overestimated due to its simplicity, two conclusions can be drawn. First, 


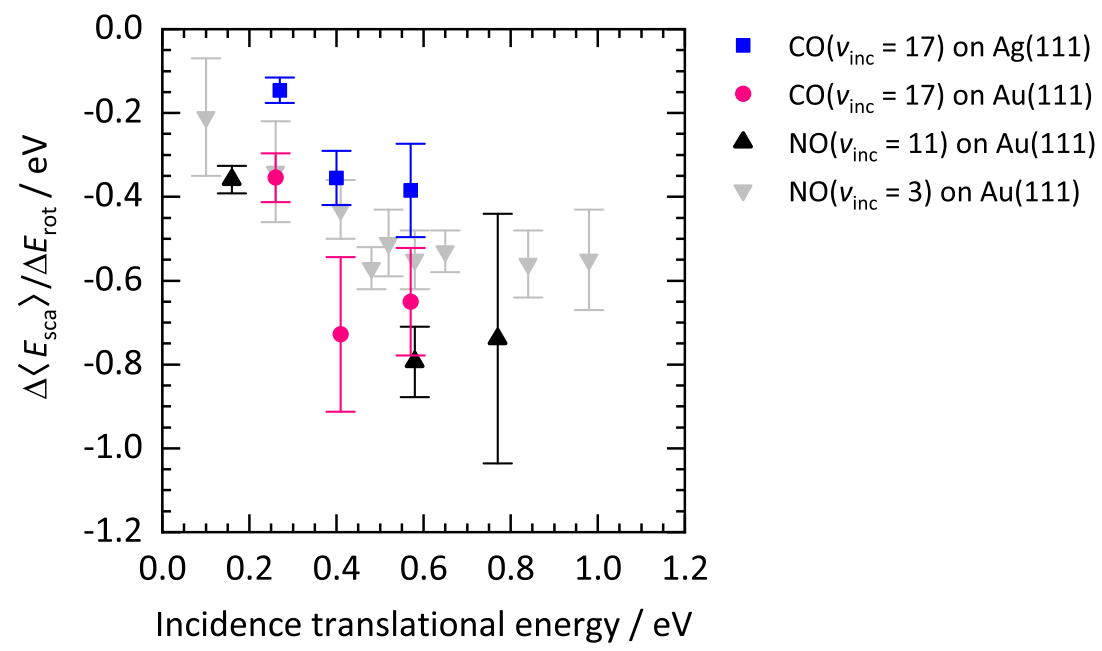

Figure 5.5: $\Delta\left\langle E_{\text {sca }}\right\rangle / \Delta E_{\text {rot }}$ as a function of incidence translational energy $\left\langle E_{\text {inc }}\right\rangle$ for vibrationally elastic scattering of $\mathrm{CO}\left(v_{\mathrm{inc}}=17\right)$ from gold (red) and silver (blue). For comparison, data are also shown for the $\mathrm{NO}\left(v_{\text {inc }}=3\right) / \mathrm{Au}(111)$ system [134] and the $\mathrm{NO}\left(v_{\text {inc }}=11\right) / \mathrm{Au}(111)$ system [129].

the good agreement with the simple Baule limit suggests that the transfer of translational energy to the surface proceeds via a mechanical, electronically adiabatic pathway. Second, the linear dependence of $\left\langle E_{\text {sca }}\right\rangle$ on $\left\langle E_{\text {inc }}\right\rangle$ gives rise to a direct scattering mechanism.

Rotationally Inelastic Scattering This subsection discusses the T-R energy transfer in vibrationally elastic scattering. Figure 5.2 shows the final translational energy $\left\langle E_{\text {sca }}\right\rangle$ of $\mathrm{CO}\left(v_{\text {inc }}=17 \rightarrow v_{\text {sca }}=17\right)$ as a function of the final rotational energy $E_{\text {rot }}$ for three different incidence translational energies $\left\langle E_{\text {inc }}\right\rangle$. The reader is reminded of two trends that are observed. First, $\left\langle E_{\text {sca }}\right\rangle$ decreases with increasing $E_{\text {rot }}$, suggesting that the rotational energy of the molecule originates from the conversion of translational energy. Second, the absolute value of the slope of linear fits plotted through the data points, $\Delta\left\langle E_{\text {sca }}\right\rangle / \Delta E_{\text {rot }}$, increases with $\left\langle E_{\text {inc }}\right\rangle$.

In Figure 5.5, $\Delta\left\langle E_{\text {sca }}\right\rangle / \Delta E_{\text {rot }}$ is plotted as a function of $\left\langle E_{\text {inc }}\right\rangle$ for different moleculesurface systems. The results obtained for highly vibrationally excited $\mathrm{CO}$ scattered from gold (red) and silver (blue) show a similar trend as data previously reported on the $\mathrm{NO}\left(v_{\text {inc }}=3\right) / \mathrm{Au}(111)$ system [134] (gray) and the $\mathrm{NO}\left(v_{\text {inc }}=11\right) / \mathrm{Au}(111)$ system [129] (black). The Figure demonstrates the increasing steepness of the slope with $\left\langle E_{\text {inc }}\right\rangle$. 
In order to understand the physical meaning of $\Delta\left\langle E_{\text {sca }}\right\rangle / \Delta E_{\text {rot }}$, it is helpful to consider to limiting cases [81, 162]. First, the slope would be -1 if there was pure energy conservation for the T-R energy transfer, implying that the sum of the final translational energy and the rotational energy of the molecule is a constant, $\left\langle E_{\mathrm{sca}}\right\rangle+E_{\text {rot }}=$ constant, and that the energy transferred to surface phonons, $E_{\mathrm{P}}$, does not change with the rotational excitation of the molecule. Second, $\Delta\left\langle E_{\mathrm{sca}}\right\rangle / \Delta E_{\mathrm{rot}}=0$ indicates that the T-R energy transfer and the translation-to-phonon (T-P) energy transfer are anti-correlated, meaning that the sum of translational energy transferred to phonons and the rotational excitation of the molecule is a constant, $E_{\mathrm{P}}+E_{\mathrm{rot}}=$ constant. In this case, the final translational energy exhibits no dependence on the rotational excitation.

Figure 5.5 shows that the value of $\Delta\left\langle E_{\text {sca }}\right\rangle / \Delta E_{\text {rot }}$ is clearly larger than -1 at $\left\langle E_{\text {inc }}\right\rangle=$ $0.57 \mathrm{eV}$ for both the scattering from gold and from silver. A slope greater than -1 can be explained within the picture of pure T-R energy transfer if there is an anti-correlation between the T-R energy transfer and T-P energy transfer. In this mechanism, the energy transfer is sensitive on the molecular orientation during the impact [81, 162]. Imagine a head-on collision of a diatomic molecule with a surface atom at zero impact parameter. The momentum transferred from the molecule to the surface will be large, but there will be no rotational excitation. On the other hand, a slight tilt of the internuclear axis with respect to the surface normal will lead to rotational excitation and the amount of phonon excitation is decreased. The data shown in Figure 5.2 support this hypothesis. For little rotational excitation, $\left\langle E_{\text {sca }}\right\rangle$ is reproduced correctly by the hard-cube model, meaning that the translational energy lost is transferred completely to phonons. For high rotational excitation, the sum of $E_{\text {rot }}$ and $\left\langle E_{\text {sca }}\right\rangle$ equals $\left\langle E_{\text {inc }}\right\rangle$, suggesting that the translational energy only excites the molecular rotation and no energy is transferred to the surface. See for example $\mathrm{CO}$ on $\mathrm{Ag}(111)$ at $\left\langle E_{\text {inc }}\right\rangle=0.27 \mathrm{eV}$, where $E_{\text {rot }} \approx 0.24 \mathrm{eV}$ and $\left\langle E_{\text {sca }}\right\rangle=0.04 \mathrm{eV}$ (see Table 5.2). This excludes rotational excitation by phonons. However, the question may arise why $E_{\text {rot }}$ gets larger than $\left\langle E_{\text {inc }}\right\rangle$ when the fits shown in Figure 5.2 are extrapolated to the intersection with the $x$-axis. Does this suggest that phonon-to-rotation energy transfer cannot be completely neglected? This question is speculative since no data points are available for such high rotational excitations. For example, the equal importance of incidence translational energy and surface thermal motion for the rotational excitation has been reported for the $\mathrm{HCl} / \mathrm{Au}(111)$ system, which exhibits a surface temperature dependence of $E_{\text {rot }}$ [163]. However, for the scattering of $\mathrm{CO}(v=17)$ from gold and silver, rotational excitation through phonons is of minor importance because of the low kinetic energy of surface atoms at a surface temperature of $300 \mathrm{~K}$ and $400 \mathrm{~K}$, respectively.

As shown in Figure 5.5, $\Delta\left\langle E_{\text {sca }}\right\rangle / \Delta E_{\text {rot }}$ approaches zero at lower $\left\langle E_{\text {inc }}\right\rangle$. This trend 


\section{Surface Scattering of $\operatorname{COX} X^{1} \Sigma^{+}(v=17)$}

may be explained by dynamical steering, which has been shown to be important for NO scattered from metal surfaces [29, 81]. Slow molecules have a higher probability to be oriented by the attractive long-range potential in front of the surface during the scattering event. This may lead to higher values of $\Delta\left\langle E_{\text {sca }}\right\rangle / \Delta E_{\text {rot }}$ given that the energetically favored orientation leads to less rotational excitation. For example, the orientation of $\mathrm{CO}$ at $\mathrm{Ni}(111)$ affects the rotational excitation as the $\mathrm{C}$ end down configuration experiences a stronger attraction than the $\mathrm{O}$ end down configuration [164].

Figure 5.5 clearly shows that $\Delta\left\langle E_{\text {sca }}\right\rangle / \Delta E_{\text {rot }}$ is significantly higher for $\operatorname{CO}\left(v_{\text {inc }}=17\right)$ scattered from $\operatorname{Ag}(111)$ than from $\mathrm{Au}(111)$. This observation agrees with the previous discussion on the hard-cube model. As the transfer of translational energy to the surface is more efficient for silver due to its lower mass, the rotational excitation will be less efficient for silver than for gold.

Vibrationally Inelastic Scattering Figure 5.3 shows $\left\langle E_{\text {sca }}\right\rangle$ as a function of final vibrational energy for $\mathrm{CO}\left(v_{\text {inc }}=17\right)$ scattered from silver into vibrational states between $v_{\text {sca }}=14\left(E_{\mathrm{vib}}=3.55 \mathrm{eV}\right)$ and $v_{\mathrm{sca}}=17\left(E_{\mathrm{vib}}=4.20 \mathrm{eV}\right)$. The final translational energy clearly increases with vibrational energy loss. The slope $\Delta\left\langle E_{\mathrm{sca}}\right\rangle / \Delta E_{\mathrm{vib}}$ of linear fits plotted through the data points gives the translational energy gain per $1 \mathrm{eV}$ vibrational energy loss and can be regarded as an indicator for the strength of the V-T energy transfer. The steepness of the fits increases with $\left\langle E_{\text {inc }}\right\rangle . \Delta\left\langle E_{\text {sca }}\right\rangle / \Delta E_{\text {vib }}$ is found to be -0.005 for $\left\langle E_{\text {inc }}\right\rangle=0.27 \mathrm{eV}$ while it is -0.089 for $\left\langle E_{\text {inc }}\right\rangle=0.57 \mathrm{eV}$. The trend in the dependence on incidence translational energy is illustrated in Figure 5.6, showing $\Delta\left\langle E_{\mathrm{sca}}\right\rangle / \Delta E_{\mathrm{vib}}$ as a function of $\left\langle E_{\text {inc }}\right\rangle$ (blue). For comparison, results are also shown for $\mathrm{NO}\left(v_{\text {inc }}=11\right)$ (green) [129] and $\mathrm{NO}\left(v_{\text {inc }}=3\right)$ (gray) [134] scattered from gold.

The translational inelasticity of vibrationally inelastically scattered molecules has been observed previously for various molecule-surface systems [80, 165, 166]. Golibrzuch et al. investigated both the vibrational relaxation and excitation of $\mathrm{NO}\left(v_{\mathrm{inc}}=2,3\right)$ at gold using state-to-state time-of-flight techniques. The authors observe that $\left\langle E_{\text {sca }}\right\rangle$ is larger (smaller) for vibrationally relaxed (excited) molecules than for vibrationally elastically scattered molecules. This observation agrees with the results presented for highly vibrationally excited $\mathrm{CO}$. A similar trend has been observed for highly vibrationally excited $\mathrm{NO}\left(v_{\text {inc }}=11\right)$ on gold [129]. Figure 5.6 compares these molecule-surface systems, indicating that they behave quite similarly.

The mechanistic details that explain the V-T energy transfer are not fully understood [80, 129]. Several mechanisms have been proposed and discussed yet by Golibrzuch et al. However, none of them is capable of explaining comprehensively all observations that are related to the V-T energy transfer. An adiabatic, purely mechanical mech- 


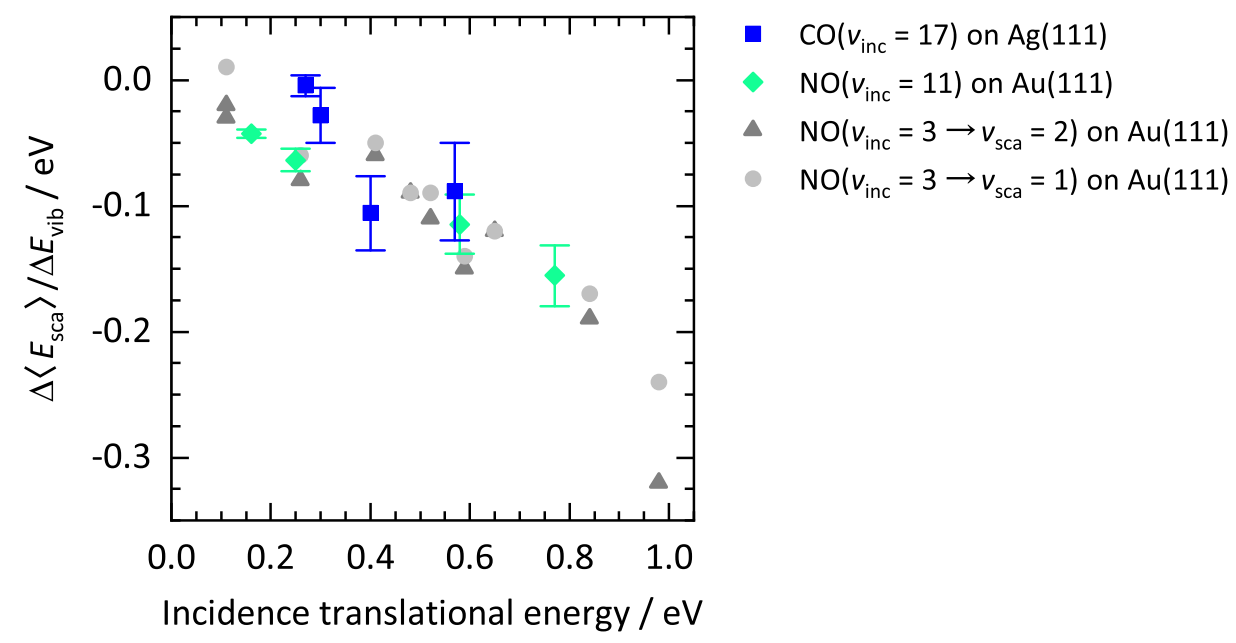

Figure 5.6: $\Delta\left\langle E_{\text {sca }}\right\rangle / \Delta E_{\text {vib }}$ as a function of incidence translational energy $\left\langle E_{\text {inc }}\right\rangle$ for $\mathrm{CO}\left(v_{\text {inc }}=17\right)$ scattered from silver (blue). For comparison, data are also shown for the $\mathrm{NO} / \mathrm{Au}(111)$ system with different initial vibrational states $[129,134]$.

anism can be ruled out because the observed V-T energy transfer is by far less than the vibrational energy loss during the collision. Complete conversion of vibrational to translational energy would be expected for an electronically adiabatic energy transfer according to experiments probing the vibrational excitation of ammonia at $\mathrm{Au}(111)$ [67]. Golibrzuch et al. propose a number of further mechanisms such as the acceleration of a transiently formed anion due to image charge stabilization, different surface site dependent reactivities, and the effect of molecular orientation at the surface. None of these mechanisms is consistent with the observed change in translational energy. However, an electronically non-adiabatic mechanism initially proposed by Rahinov et al. agrees qualitatively with the observed V-T energy transfer [166]. Here, direct coupling between the electronic degrees of freedom of the surface and the translation of the molecule is assumed. During vibrational relaxation, vibrational energy is transferred to the surface and excites an electron-hole pair (EHP), which subsequently decays partially into translation. However, this mechanism cannot explain the dependence of $\left\langle E_{\mathrm{sca}}\right\rangle$ on the surface temperature $T_{\mathrm{s}}$, which exhibits a steeper slope $\mathrm{d}\left\langle E_{\mathrm{sca}}\right\rangle / \mathrm{d} T_{\mathrm{s}}$ for vibrationally inelastic scattering than for elastic scattering [80]. Thus, certain aspects of the translational inelasticity remain unclear.

The results presented for highly vibrationally excited CO are consistent with the electronically non-adiabatic mechanism, which makes sense as the coupling of vibration 
to EHPs is well known for CO and NO at metal surfaces [25, 31, 62, 76, 77, 86]. In the following, it will be shown that the concept of image charge stabilization (ICS), which has been rejected earlier [80], is actually consistent with the non-adiabatic picture and helps to understand the nature of the V-T energy transfer.

The detailed mechanism will be explained in Section 5.3.2, which deals with the vibrational relaxation of highly vibrationally excited CO at metal surfaces. Briefly, the mechanism consists of two steps. First, the approaching molecule accepts an electron from the surface and forms a transient anion. The electron transfer happens when the affinity level of the molecule is sufficiently lowered in energy by ICS such that it is resonant with occupied states of the surface. Second, the vibrational state of the departing molecule may have changed during the scattering event, which results in the generation of an excited or relaxed EHP as soon as the electron is transferred back to the surface. Once the anion is formed, the incoming molecule is accelerated in the electric field induced by the positive image charge in the surface. Consequently, the scattered molecule is decelerated as it leaves the surface until the neutral species is reformed. The largest molecule-surface distance at which the electron transfer is energetically possible is determined by the ICS that is needed to make the affinity level resonant with occupied surface states. The required ICS depends on the work function of the surface and the electron binding energy of the molecule. The electron binding energy in turn depends on the vibrational state of the molecule. At higher electron binding energies, the required ICS is lower and the maximum molecule-surface distance at which the electron transfer is energetically feasible becomes larger. For vibrational states considered in the context of this thesis, the vertical electron binding energy at the outer classical turning point of the vibrating $\mathrm{CO}$ (or NO) molecule increases with vibrational excitation. Based on these considerations, it is possible to understand the impact of vibrational inelasticity on the final translational energy. When the vibration is de-excited during the collision event, the travel distance of the transient anion through the Coulomb potential of the image charge is shorter for the scattered molecule than for the incoming molecule. Vibrationally relaxed molecules gain translational energy as they experience a longer period of acceleration when they approach the surface than of deceleration when they leave the surface. The opposite scenario holds for the case of vibrational excitation.

The trend that $\left\langle E_{\mathrm{sca}}\right\rangle$ increases with vibrational relaxation and decreases with vibrational excitation agrees with the observations for $\mathrm{NO}(v=2,3)$ scattered from gold [80]. The proposed mechanism also agrees with the trend that the translational energy gain increases with the number of relaxed vibrational quanta, as observed for CO and NO initially prepared in high $v$ states. However, the mechanism is still not capable of explaining the aforementioned effect of surface temperature on the translational 
(A)

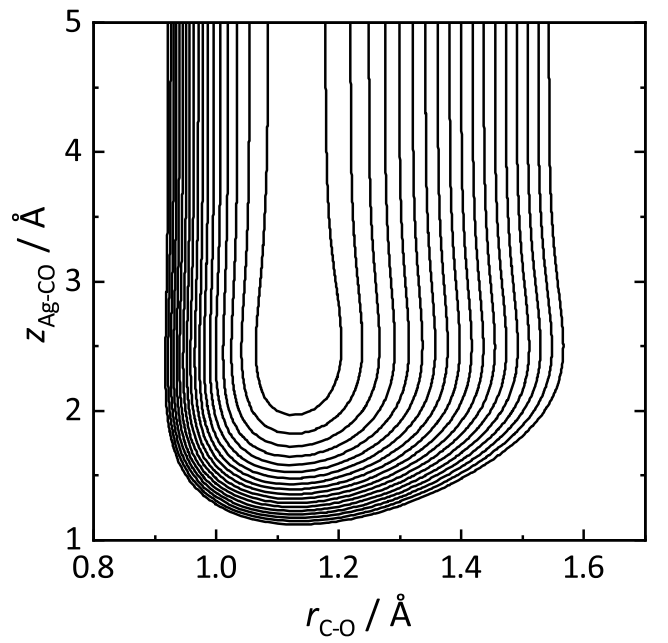

(B)

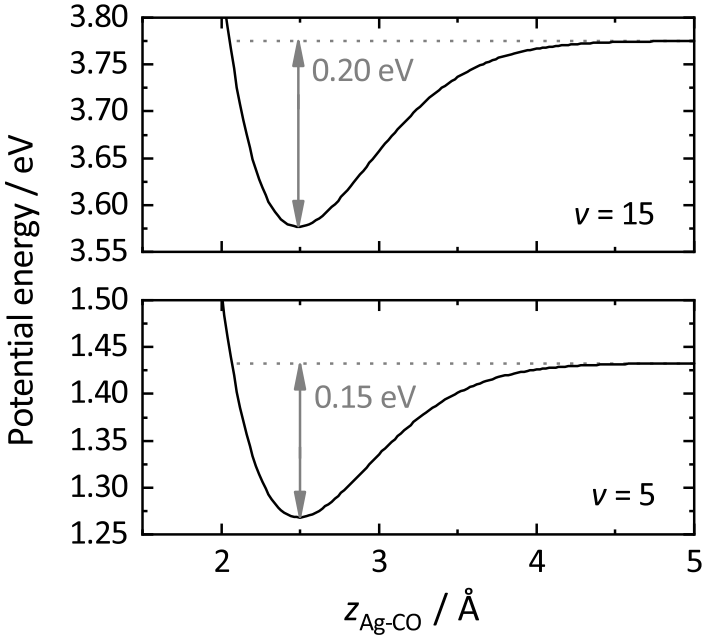

Figure 5.7: Panel (A) shows the electronically adiabatic potential energy surface (PES) of the $\mathrm{CO} / \mathrm{Ag}(111)$ system as a function of the internuclear distance $r_{\mathrm{C}-\mathrm{O}}$ and the molecule-surface distance $z_{\mathrm{Ag}-\mathrm{CO}}$. The $\mathrm{CO}$ approaches the hcp site of the surface with the $\mathrm{C}$ end pointing towards the surface and the $\mathrm{CO}$ bond axis parallel to the surface normal. The energy of the depicted contours correspond to the vibrational energies of gas-phase CO infinitely far from the surface ranging from $v=0$ to $v=17$. Detailed information on the calculation of the $\mathrm{CO} / \mathrm{Ag}(111)$ PES will be provided in Section 5.3.3. Panel (B) shows the vibrationally adiabatic potential energy of $\mathrm{CO}(v=5)$ and $\mathrm{CO}(v=15)$ as a function of $z_{\mathrm{Ag}-\mathrm{CO}}$.

inelasticity.

The qualitative understanding of the V-T energy transfer is supported by a closer look at the potential energy surface (PES) of the molecule-surface system, which is shown in Figure 5.7. Computational details on the PES are provided in Section 5.3.3. Panel (A) shows the electronically adiabatic potential energy surface of the $\mathrm{CO} / \mathrm{Ag}(111)$ system as a function of the internuclear distance $r_{\mathrm{C}-\mathrm{O}}$ and the molecule-surface distance $z_{\mathrm{Ag}-\mathrm{CO}}$. The isoenergetic contours correspond to the vibrational energies of gas-phase $\mathrm{CO}(0 \leq v \leq 17)$ infinitely far from the surface. Panel (B) shows the vibrationally adiabatic $\mathrm{PES}$ of $\mathrm{CO}(v=5)$ (lower panel) and $\mathrm{CO}(v=15)$ (upper panel) as a function of $z_{\text {Ag-CO }}$. Vibrationally adiabatic PESs are obtained by fitting the internuclear potential for each value of $z_{\mathrm{Ag}-\mathrm{CO}}$, which corresponds to slices through the PES shown in Panel 
(A) at the respective $z_{\mathrm{Ag}-\mathrm{CO}}$, and calculating the vibrational energy associated with this internuclear potential. The curves shown in Panel (B) exhibit a potential energy well that originates from the anionic character of the PES at small $z_{\mathrm{Ag}-\mathrm{CO}}$ and the associated ICS. Note that the well depth increases slightly with vibrational excitation, which agrees with the proposed mechanism. The higher the vibrational excitation, the further away from the surface the molecule may accept an electron. CO in a high $v$ state experiences the field of the image charge longer than CO in a low $v$ state, no matter whether the molecule approaches or leaves the surface. If, however, the vibrational state changes during the scattering event, the well depth of the departing molecule differs from the one of the incoming molecule, which directly affects the translational energy. The potential energy well is deeper for vibrationally excited molecules which, thus, lose translational energy. On the other hand, the energy well is shallower for vibrationally relaxed molecules which results in a translational energy gain.

It has been demonstrated that the translational inelasticity in vibrationally inelastic scattering is likely to obey electronically non-adiabatic dynamics. Concepts of transient anion formation and image charge stabilization help explain the nature of the EHP-V and EHP-T interactions. It will be shown in Chapter 5.3 that the proposed mechanism is fundamentally important for the dynamics of diatomics at metal surfaces because it helps explain the final vibrational state distributions of surface scattered $\operatorname{CO}\left(v_{\text {inc }}=17\right)$.

\subsection{Angular Distributions}

This section presents the angular distributions measured for highly vibrationally excited CO scattered from $\mathrm{Au}(111)$ and $\mathrm{Ag}(111)$. Together with time-of-flight measurements, angular distributions provide valuable information about the scattering mechanism and help to distinguish between direct scattering and trapping-desorption [167]. Assuming a smooth surface potential, direct scattering is indicated by a narrow $\cos ^{n}(\theta)$ distribution with $n \gg 1$, while trapping-desorption is characterized by a broad distribution with $n \approx 1$ peaking at the surface normal.

Figures 5.8 and 5.9 show angular distributions of $\mathrm{CO}\left(v_{\text {inc }}=17\right)$ scattered vibrationally elastically from gold and silver, respectively. The signal is corrected for background signal arising from Franck-Condon pumping. Results are shown for incidence translational energies ranging from $0.26 \mathrm{eV}$ to $0.57 \mathrm{eV}$. The arrow indicates the incidence angle of the molecular beam. The cosine distribution given in Equation 4.11 is used to fit the experimental data (solid lines).

Two observations are striking. First, the comparison of the incidence angle $\theta_{\text {inc }}$ with the scattering angle $\theta_{\text {sca }}$ at which the cosine distribution peaks suggests specular scat- 


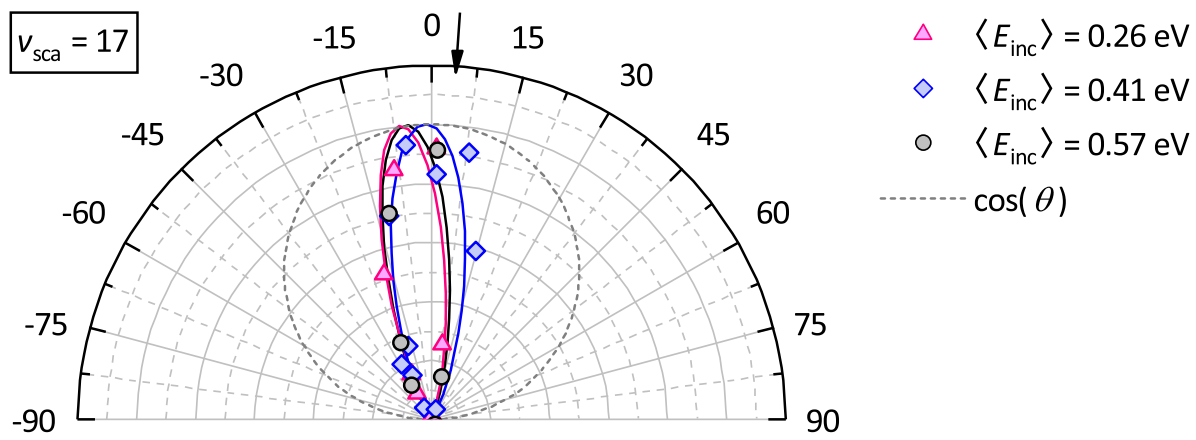

Figure 5.8: Normalized angular distributions of $\mathrm{CO}\left(v_{\text {inc }}=17\right)$ scattered vibrationally elastically from $\mathrm{Au}(111)$. Results are shown for three different incidence translational energies. The surface temperature is $T_{\mathrm{s}}=300 \mathrm{~K}$. The arrow marks the incidence angle at $\theta_{\text {inc }}=3^{\circ}$. A $\cos ^{n}(\theta)$ distribution is fitted through the data points (solid lines) using Equation 4.11. The exponent $n$ varies between 23 and 30 .

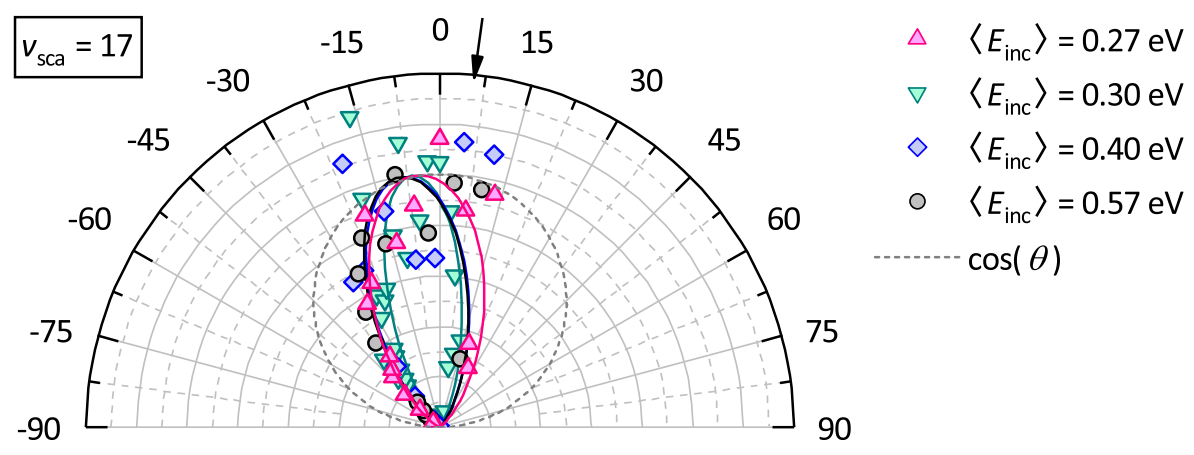

Figure 5.9: Normalized angular distributions of $\mathrm{CO}\left(v_{\text {inc }}=17\right)$ scattered vibrationally elastically from $\operatorname{Ag}(111)$. Results are shown for four different incidence translational energies. The surface temperature is $T_{\mathrm{s}}=400 \mathrm{~K}$. The arrow marks the incidence angle at $\theta_{\mathrm{inc}}=5^{\circ} . \mathrm{A} \cos ^{n}(\theta)$ distribution is fitted through the data points (solid lines) using Equation 4.11. The exponent $n$ varies between 7 and 17 .

tering. Second, the fitting procedure yields $23 \leq n \leq 30$ for gold and $7 \leq n \leq 17$ for silver, indicating that the observed angular distributions are rather narrow. For comparison, a $\cos ^{1}(\theta)$ distribution (gray dashed) is also shown in the Figures. These two observations suggest that vibrationally elastically scattered molecules follow a direct 


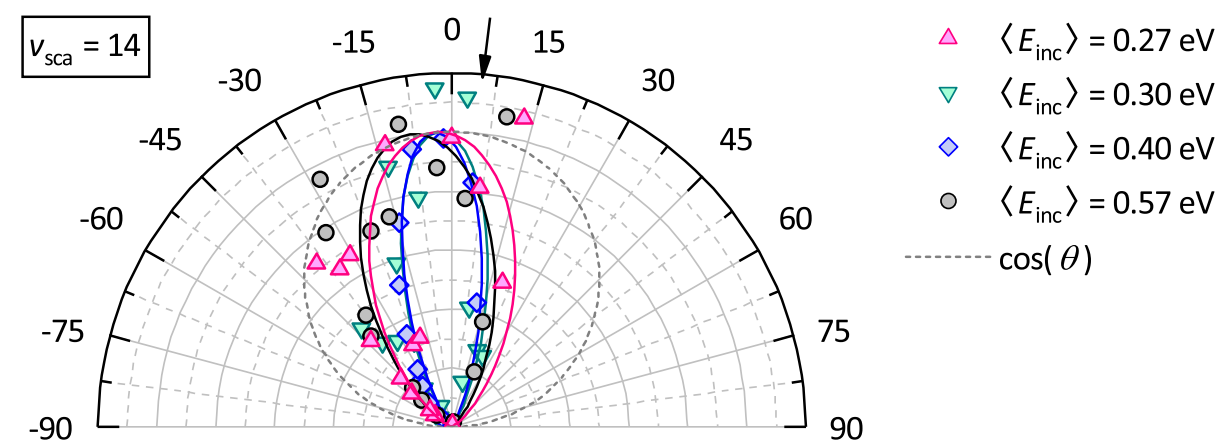

Figure 5.10: Normalized angular distributions of $\mathrm{CO}\left(v_{\text {inc }}=17\right)$ scattered vibrationally inelastically from $\operatorname{Ag}(111)$ into $v_{\text {sca }}=14$. Results are shown for four different incidence translational energies. The surface temperature is $T_{\mathrm{s}}=400 \mathrm{~K}$. The arrow marks the incidence angle at $\theta_{\text {inc }}=5^{\circ} . \mathrm{A}$ $\cos ^{n}(\theta)$ distribution is fitted through the data points (solid lines) using Equation 4.11. The exponent $n$ varies between 6 and 20 .

scattering mechanism. This conclusion is consistent with the observation that $\left\langle E_{\text {sca }}\right\rangle$ of vibrationally elastically scattered $\mathrm{CO}$ increases linearly with $\left\langle E_{\text {inc }}\right\rangle$ (see Section 5.1.2). The observation that the angular distribution seems to be broader for silver than for gold might be a consequence of the more efficient transfer of linear momentum to silver than to gold (see Section 5.1.2).

Angular distributions for $\mathrm{CO}\left(v_{\text {inc }}=17 \rightarrow v_{\text {sca }}=14\right)$ scattering on silver are shown in Figure 5.10. The angular distributions of vibrationally inelastic scattering are also consistent with a direct scattering mechanism.

It can be concluded that in both the vibrationally elastic and inelastic channels, highly vibrationally excited $\mathrm{CO}$ with $\left\langle E_{\mathrm{inc}}\right\rangle \geq 0.26 \mathrm{eV}$ scatters directly from both $\mathrm{Au}(111)$ and $\operatorname{Ag}(111)$.

\subsection{Vibrational State Distributions}

This section presents the REMPI spectra of surface scattered CO and discusses the resulting final vibrational state distributions. In the following, results are shown for the $\mathrm{CO} / \mathrm{Au}(111)$ system [31] first and then for the $\mathrm{CO} / \mathrm{Ag}(111)$ system [32]. These systems are characterized by different work functions. Scattering results are also presented for a chlorinated $\mathrm{Ag}(111)$ surface, which exhibits a work function $1.5 \mathrm{eV}$ higher than that of clean $\operatorname{Ag}(111)$. The comparison to previously studied molecule-surface systems suggests 
that an electron transfer process is essential to the vibrational relaxation of diatomic molecules at metal surfaces.

\subsubsection{Results}

Surface Scattering from Au(111) $(1+1)$ REMPI spectra via the $\mathrm{A}^{1} \Pi\left(v^{\prime}, J^{\prime}\right) \leftarrow$ $\mathrm{X}^{1} \Sigma^{+}\left(v^{\prime \prime}, J^{\prime \prime}\right)$ transition ranging from $41000 \mathrm{~cm}^{-1}(244 \mathrm{~nm})$ to $43800 \mathrm{~cm}^{-1}(228 \mathrm{~nm})$ have been recorded to probe the vibrational states $14 \leq v_{\text {sca }} \leq 17$ in the surface scattered beam. Figure 5.11 shows spectra of highly vibrationally excited CO scattered from $\mathrm{Au}(111)$ for three different $\left\langle E_{\text {inc }}\right\rangle$. Vibrational bands are labeled by $v^{\prime}-v^{\prime \prime}$, where $v^{\prime \prime}$ is the detected vibrational state. Results are shown for the dump laser turned on (black) and turned off (orange). The reader is reminded that the dump laser selectively increases the population of $\operatorname{CO}\left(v_{\text {inc }}=17, J_{\text {inc }}=0\right)$ in the incoming beam. The signal obtained after subtracting the orange trace from the black trace corresponds to the signal that originates only from incident $\mathrm{CO}$ prepared in the single rovibrational state $\mathrm{X}^{1} \Sigma^{+}\left(v_{\text {inc }}=17, J_{\text {inc }}=0\right)$. Thus, the orange trace can be regarded as a background signal which originates from various rovibrational states present in the incident beam due to Franck-Condon pumping.

Three conclusions can be drawn from inspection of the spectra shown in Figure 5.11. First, highly vibrationally excited CO undergoes vibrational relaxation when colliding with a gold surface because population in $v_{\text {sca }}<17$ is observed. Second, the population in relaxed vibrational states increases with $\left\langle E_{\text {inc }}\right\rangle$, suggesting that the vibrational relaxation probability increases with $\left\langle E_{\text {inc }}\right\rangle$. Third, a closer look at the rotational structure of the vibrational bands reveals that the rotational temperature increases with $\left\langle E_{\text {inc }}\right\rangle$. Together with the linear dependence of $\left\langle E_{\mathrm{sca}}\right\rangle$ on $\left\langle E_{\mathrm{inc}}\right\rangle$ (Section 5.1) and the narrow angular distribution observed for vibrationally relaxed scattering products (Section 5.2), the increase in rotational excitation with $\left\langle E_{\text {inc }}\right\rangle$ provides further evidence for a direct scattering mechanism. Final rotational state distributions are presented in Section 5.4.

Figure 5.12 shows the final vibrational state distributions that are derived from the REMPI spectra following the analysis described in Section 4.3. The plots demonstrate that highly vibrationally excited $\mathrm{CO}$ molecules scatter mainly vibrationally elastically from $\mathrm{Au}(111)$ and, thus, retain their vibrational energy. For $\left\langle E_{\text {inc }}\right\rangle=0.26 \mathrm{eV}, 20 \%$ of the molecules suffer vibrational relaxation. For $\left\langle E_{\text {inc }}\right\rangle=0.57 \mathrm{eV}$, the fraction of vibrationally relaxed molecules increases to $40 \%$. However, less than $5 \%$ of the molecules relax down to $v_{\text {sca }}=14$ for any $\left\langle E_{\text {inc }}\right\rangle$. It is concluded that vibrational relaxation to the levels $v_{\text {sca }}<14$ is very unlikely to occur and can be neglected in the determination of vibrational relaxation probabilities. This hypothesis is supported by a calculation 
that compares the number of incident $\mathrm{CO}\left(v_{\text {inc }}=17\right)$ with the number of molecules scattered into $14 \leq v_{\text {sca }} \leq 17$. Following the analysis described in Section 4.3, it can be shown that the amount of incident $\mathrm{CO}\left(v_{\mathrm{inc}}=17\right)$ equals the amount of scattered $\mathrm{CO}\left(14 \leq v_{\text {sca }} \leq 17\right)$ for $\left\langle E_{\text {inc }}\right\rangle=0.57 \mathrm{eV}$. Thus, relaxation to lower vibrational states is not expected. Note that the relaxation probability decreases as $\left\langle E_{\text {inc }}\right\rangle$ is decreased

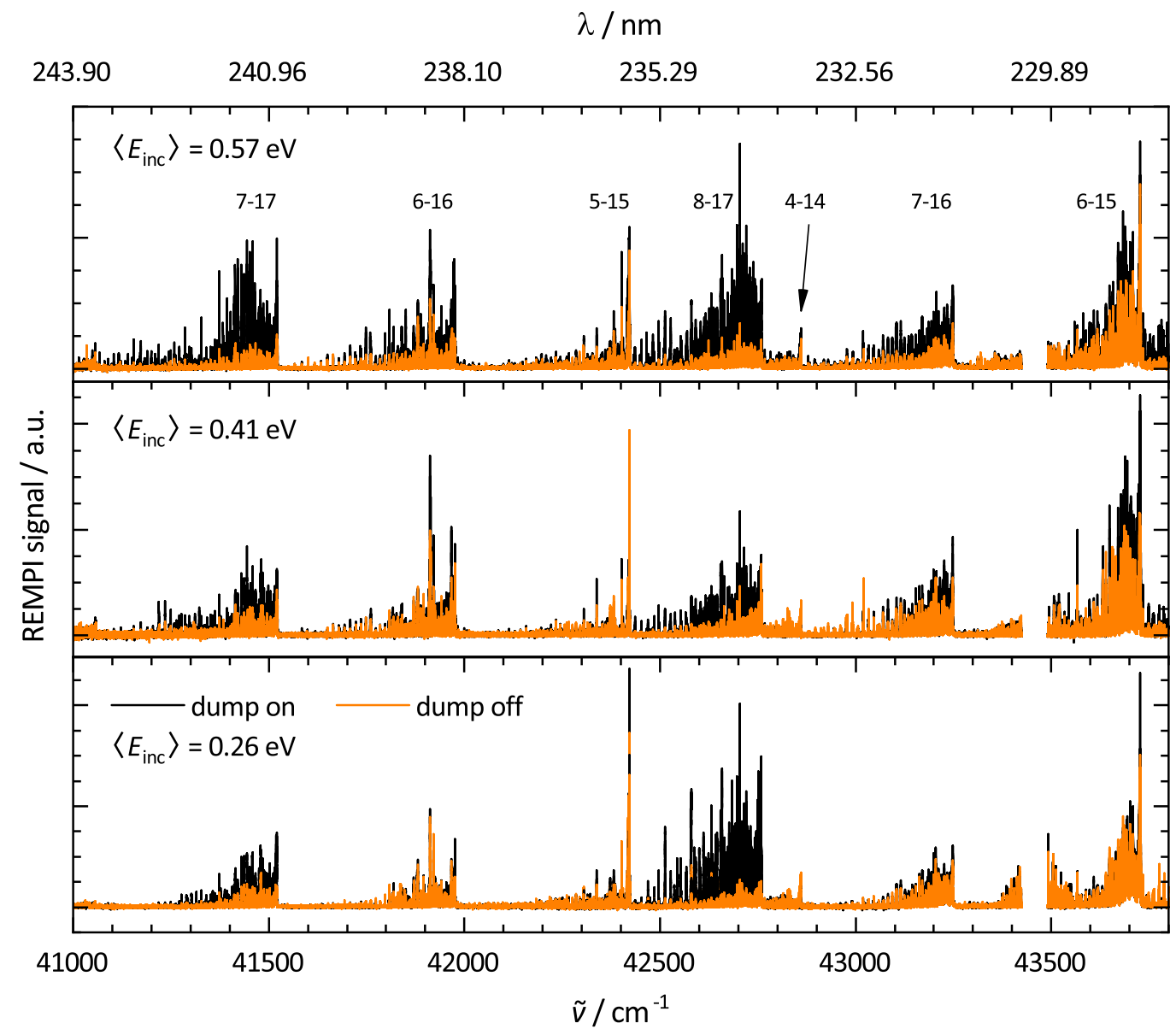

Figure 5.11: $(1+1)$ REMPI spectra via $\mathrm{A}^{1} \Pi\left(v^{\prime}, J^{\prime}\right) \leftarrow \mathrm{X}^{1} \Sigma^{+}\left(v^{\prime \prime}, J^{\prime \prime}\right)$ are shown for highly vibrationally excited $\mathrm{CO}$ scattered from $\mathrm{Au}(111)$ at three incidence translational energies $\left\langle E_{\text {inc }}\right\rangle$. Spectra are shown with the dump laser turned on (black) and turned off (orange). The dump laser selectively increases the population of $\operatorname{CO}\left(v_{\text {inc }}=17, J_{\text {inc }}=0\right)$ in the incoming beam. The spectra are corrected for fluctuations in the probe laser power. Vibrational bands are labeled by $v^{\prime}-v^{\prime \prime}$. 
and that $\left\langle E_{\text {inc }}\right\rangle=0.57 \mathrm{eV}$ is the highest incidence translational energy investigated. Consequently, the assumption also holds for lower $\left\langle E_{\text {inc }}\right\rangle$. The fact that the relaxation probability varies with $\left\langle E_{\text {inc }}\right\rangle$ can be seen in Figure 5.12, as the population of $v_{\text {sca }}=17$ clearly decreases with $\left\langle E_{\text {inc }}\right\rangle$ while the population of $v_{\text {sca }}=16$ increases. The trend is
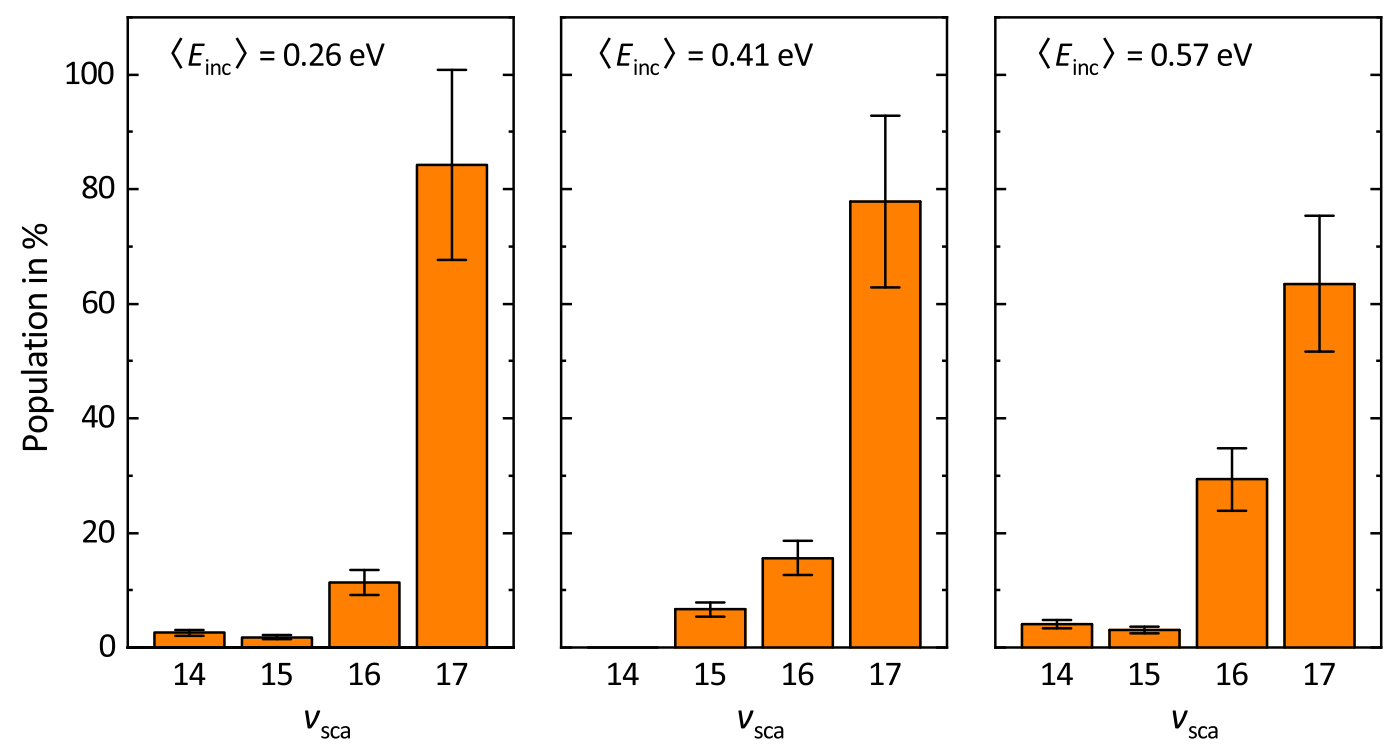

Figure 5.12: Final vibrational state distributions of $\mathrm{CO}\left(v_{\text {inc }}=17\right)$ scattered from $\mathrm{Au}(111)$ for three different incidence translational energies $\left\langle E_{\mathrm{inc}}\right\rangle$.

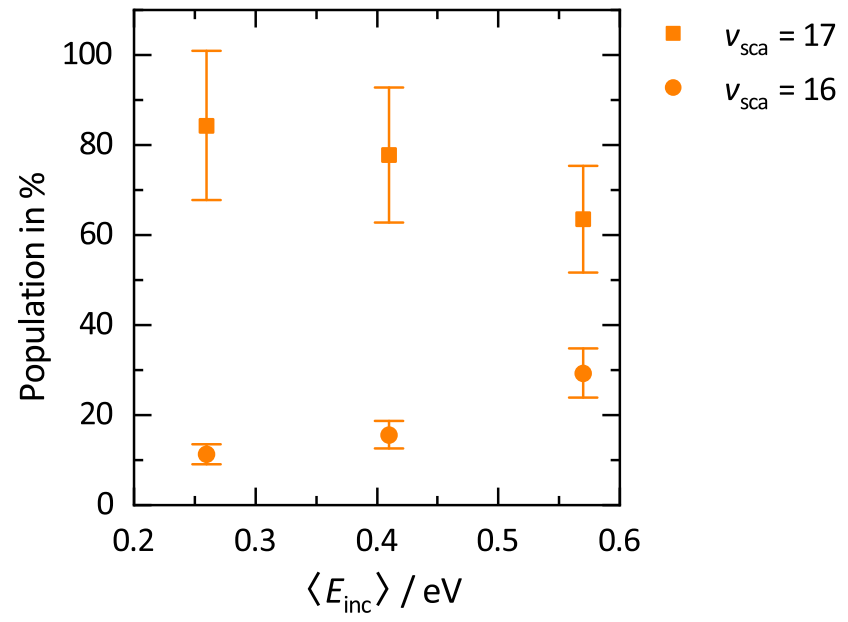

Figure 5.13: Population of $v_{\text {sca }}=16$ and 17 in $\%$ as a function of incidence translational energy $\left\langle E_{\text {inc }}\right\rangle$. 


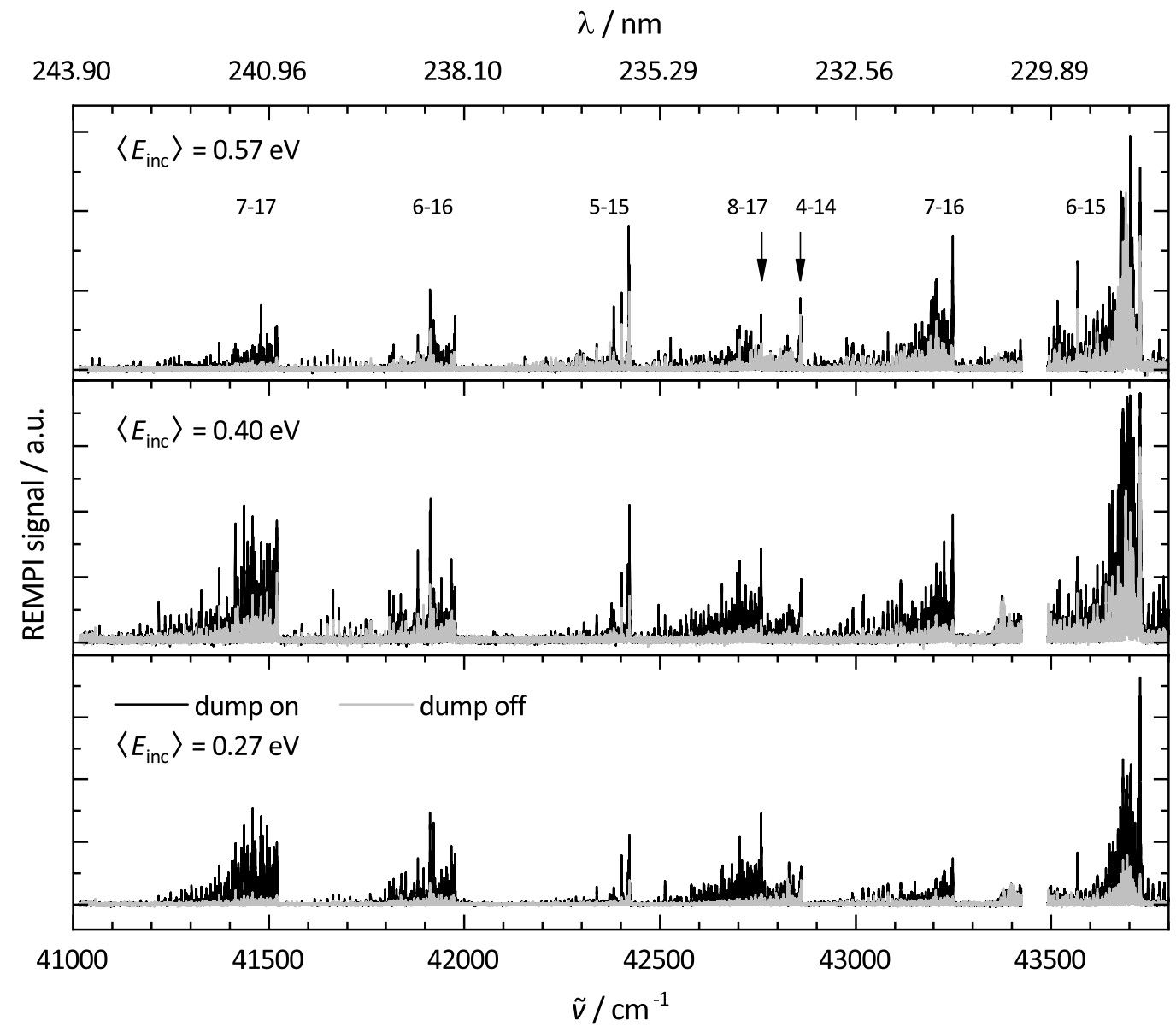

Figure 5.14: $(1+1)$ REMPI spectra via $\mathrm{A}^{1} \Pi\left(v^{\prime}, J^{\prime}\right) \leftarrow \mathrm{X}^{1} \Sigma^{+}\left(v^{\prime \prime}, J^{\prime \prime}\right)$ are shown for highly vibrationally excited $\mathrm{CO}$ scattered from $\mathrm{Ag}(111)$ at three incidence translational energies $\left\langle E_{\text {inc }}\right\rangle$. Spectra are shown with the dump laser turned on (black) and turned off (gray). The dump laser selectively increases the population of $\mathrm{CO}\left(v_{\mathrm{inc}}=17, J_{\mathrm{inc}}=0\right)$ in the incoming beam. The spectra are corrected for fluctuations in the probe laser power. Vibrational bands are labeled by $v^{\prime}-v^{\prime \prime}$.

illustrated more clearly by Figure 5.13 which shows the population of $v_{\text {sca }}=16$ and 17 as a function of $\left\langle E_{\text {inc }}\right\rangle$.

Surface Scattering from $\mathbf{A g}(\mathbf{1 1 1}) \quad(1+1)$ REMPI spectra via the $\mathrm{A}^{1} \Pi\left(v^{\prime}, J^{\prime}\right) \leftarrow$ $\mathrm{X}^{1} \Sigma^{+}\left(v^{\prime \prime}, J^{\prime \prime}\right)$ transition are presented in Figure 5.14 for highly vibrationally excited CO scattered from $\operatorname{Ag}(111)$. Similar trends can be observed as for the scattering from 

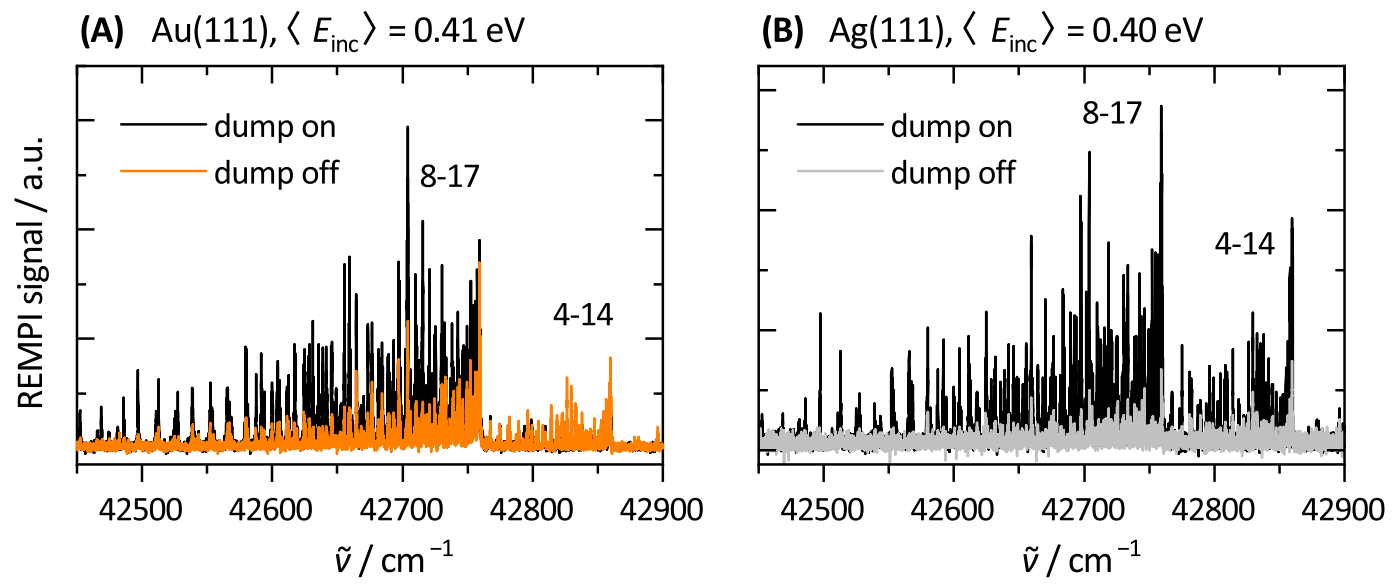

Figure 5.15: $(1+1)$ REMPI spectra via $\mathrm{A}^{1} \Pi\left(v^{\prime}, J^{\prime}\right) \leftarrow \mathrm{X}^{1} \Sigma^{+}\left(v^{\prime \prime}, J^{\prime \prime}\right)$ of highly vibrationally excited CO scattered from gold, Panel (A), and silver, Panel (B). The vibrational bands 8-17 and 4-14 are probed. Spectra are shown for the dump laser turned on (black) and turned off (orange/gray).

gold. However, the spectra indicate that the vibrational relaxation is more efficient for silver than for gold. This is emphasized in Figure 5.15, which directly compares the vibrationally elastic channel with the vibrationally inelastic channel by contrasting the relative intensities of the 8-17 band and the 4-14 band in the scattering distribution at the two surfaces. Panel (A) and (B) show results obtained for the scattering from gold and silver, respectively, at similar incidence translational energies. Note that the intensity of the 4-14 band relative to the 8-17 band is stronger for silver than for gold. Furthermore, the difference between the "dump on" spectrum and the "dump off" spectrum of the 4-14 band is larger for silver than for gold. Both of these observations indicate that the population in $v_{\text {sca }}=14$ relative to the population in $v_{\text {sca }}=17$ is greater when highly vibrationally excited $\mathrm{CO}$ is scattered from silver.

The quantitative analysis of the spectra yields the final vibrational state distributions shown in Figure 5.16. The strong vibrational inelasticity is indicated by the small population of $v_{\text {sca }}=17.85 \%(78 \%)$ of the incident molecules experience vibrational relaxation for $\left\langle E_{\text {inc }}\right\rangle=0.57 \mathrm{eV}(0.27 \mathrm{eV})$. The incidence translational energy dependence of the relaxation probability is also observed for the scattering from silver but it is not as strong as that observed for gold.

It is also striking that the vibrational energy loss appears to be larger for silver than for gold. For scattering from silver, the number of incident $\mathrm{CO}\left(v_{\text {inc }}=17\right)$ molecules significantly exceeds the number of scattered molecules detected in the range $14 \leq$ 

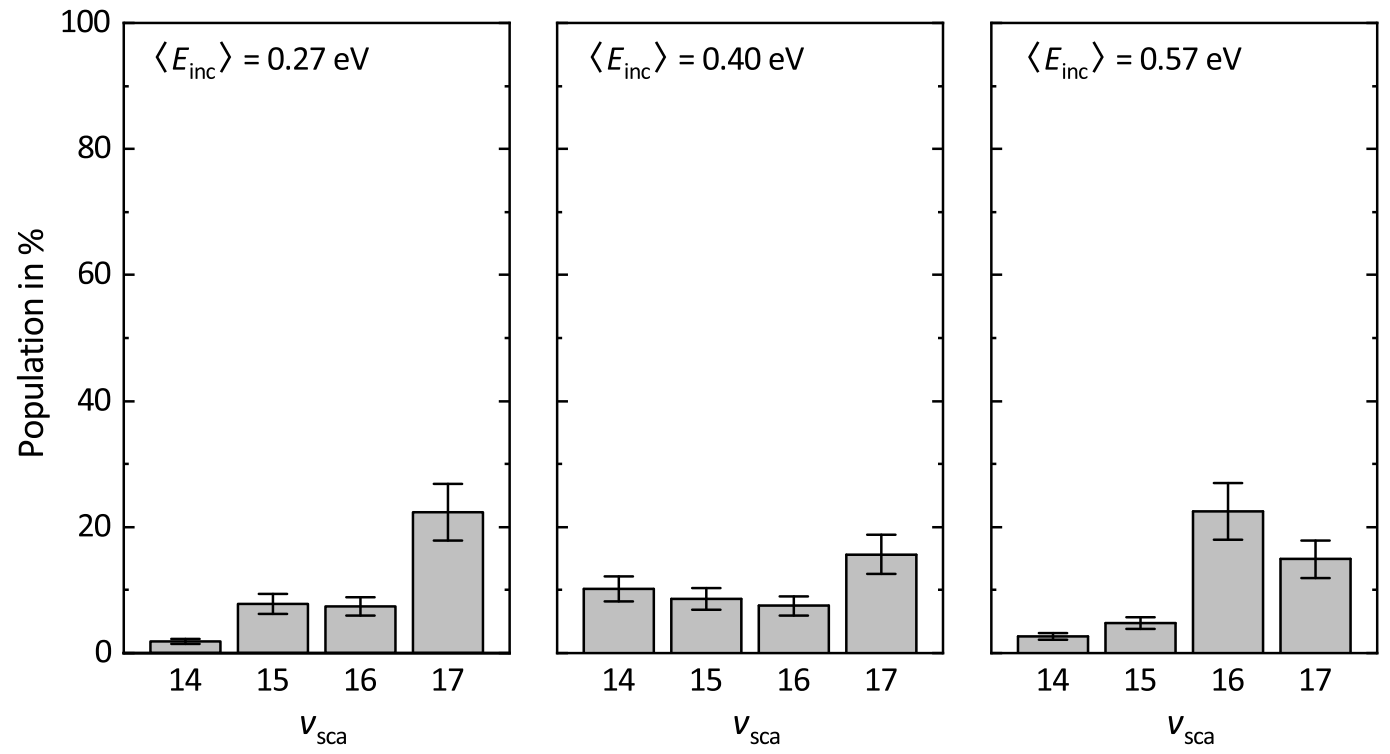

Figure 5.16: Final vibrational state distributions of $\mathrm{CO}\left(v_{\text {inc }}=17\right)$ scattered from $\operatorname{Ag}(111)$ for three different incidence translational energies $\left\langle E_{\text {inc }}\right\rangle$.

$v_{\text {sca }} \leq 17$. This suggests that the scattered molecules also populate vibrational states lower than 14 although $v_{\text {sca }}<14$ cannot be detected directly due to limitations in the REMPI scheme.

Surface Scattering from Chlorinated $\mathbf{A g}(\mathbf{1 1 1})$ In order to understand the influence of the work function on the vibrational relaxation probability, a chlorinated $\operatorname{Ag}(111)$ surface was prepared by dosing molecular chlorine onto $\mathrm{Ag}(111)$. The chlorinated surface exhibits a work function which is $1.5 \mathrm{eV}$ higher than that of atomically clean $\mathrm{Ag}(111)$ [144]. Even though the chlorinated surface is not as well characterized as the (111) face of a metal single crystal, beam-surface scattering experiments reveal that the scattering of highly vibrationally excited $\mathrm{CO}$ from chlorinated silver proceeds via a direct scattering mechanism. This is demonstrated by the angular distributions shown in Panel (B) of Figure 5.17, which are as narrow as the ones obtained for clean $\mathrm{Ag}(111)$ shown in Figure 5.9. In addition, time-of-flight experiments indicate that the final translational energy of vibrationally elastically scattered molecules obeys the Baule limit calculated for an average mass of one silver atom and one chlorine atom.

The REMPI spectra in Panel (A) of Figure 5.17 show the 8-17 band and the 4-14 of highly vibrationally excited $\mathrm{CO}$ scattered from chlorinated $\mathrm{Ag}(111)$. The spectra show that a large amount of $\mathrm{CO}\left(v_{\mathrm{inc}}=17\right)$ survives the collision with the surface, while 
(A)

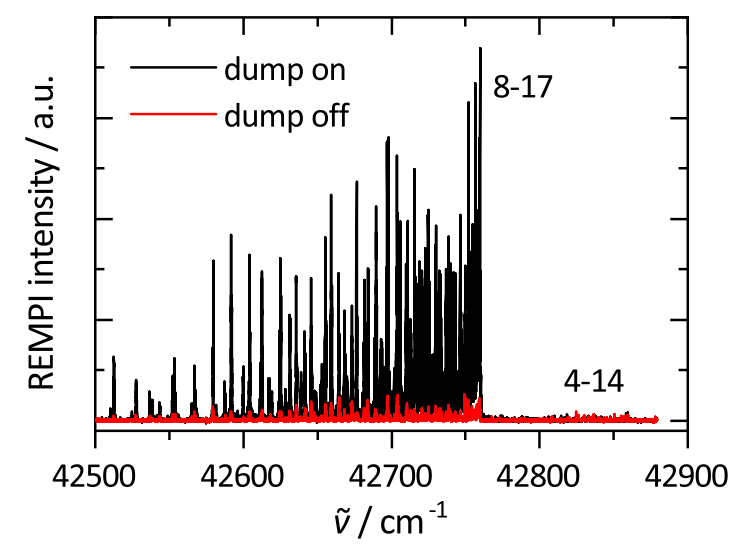

(B)



Figure 5.17: Panel (A) shows the $(1+1) \mathrm{A}^{1} \Pi\left(v^{\prime}, J^{\prime}\right) \leftarrow \mathrm{X}^{1} \Sigma^{+}\left(v^{\prime \prime}, J^{\prime \prime}\right)$ REMPI spectra of highly vibrationally excited CO scattered from a chlorinated $\mathrm{Ag}(111)$ surface for dump on (black) and dump off (red). The spectrum contains the 8-17 band and the 4-14 band, the latter of which is almost not existent. The incidence translational energy is $\left\langle E_{\text {inc }}\right\rangle=0.57 \mathrm{eV}$. Panel (B) shows the angular distribution of $\mathrm{CO}\left(v_{\text {inc }}=17\right)$ scattered vibrationally elastically from a chlorinated $\mathrm{Ag}(111)$ surface (red open circles). A cosine distribution is fitted to the experimental data using Equation 4.11 (black solid). The exponent is $n=9.9$, indicating a direct scattering mechanism. The arrow marks the incidence angle $\theta_{\text {inc }}=5^{\circ}$. For comparison, a cosine distribution with $n=1$ is plotted, too (gray dashed).

relaxation to $v_{\text {sca }}=14$ is absent. Investigations of the $6-15$ band and the 7-16 band show that the probability for vibrational relaxation to $v_{\text {sca }}=15$ and 16 is extremely small. Figure 5.18 shows the final vibrational state distribution for $\mathrm{CO}\left(v_{\text {inc }}=17\right)$ scattered from chlorinated silver and compares the results with those obtained for clean $\operatorname{Ag}(111)$ and $\mathrm{Au}(111)$. For $\left\langle E_{\text {inc }}\right\rangle=0.57 \mathrm{eV}$, the survival probability for highly vibrationally excited $\mathrm{CO}$ is $96 \%$ for chlorinated silver, $63 \%$ for clean $\mathrm{Au}(111)$, and $15 \%$ for clean $\mathrm{Ag}(111)$. These results suggest that the vibrational relaxation probability increases with decreasing work function of the surface. In the following, these findings will be discussed in terms of a mechanism that involves an electron transfer between surface and molecule. 

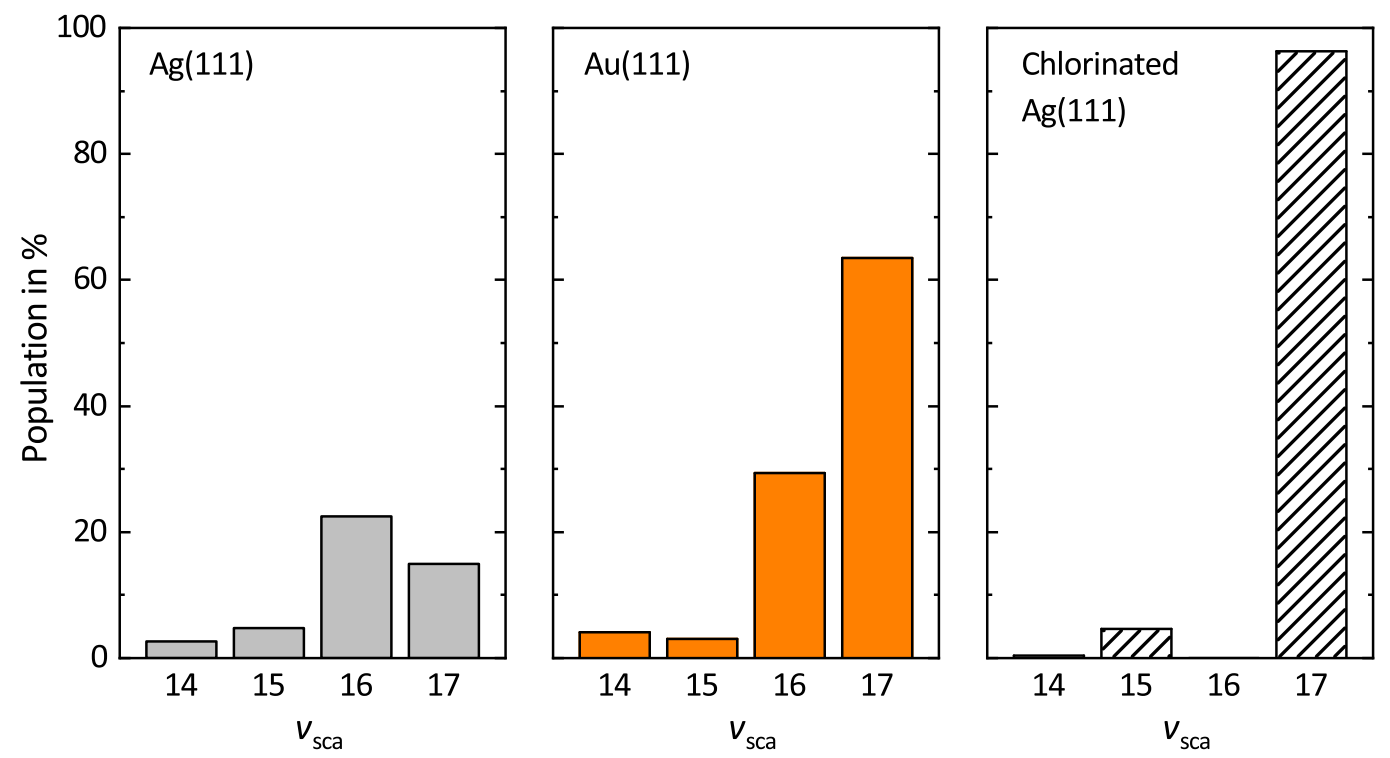

Figure 5.18: Final vibrational state distributions of $\mathrm{CO}\left(v_{\text {inc }}=17\right)$ scattered from $\mathrm{Ag}(111), \mathrm{Au}(111)$, and chlorinated $\mathrm{Ag}(111)$ at $\left\langle E_{\text {inc }}\right\rangle=0.57 \mathrm{eV}$.

\subsubsection{Vibrational Relaxation Mechanism}

In collisions with metal surfaces, such as $\mathrm{Au}(111)$ and $\mathrm{Ag}(111)$, the vibrational excitation or relaxation of diatomic molecules, such as $\mathrm{NO}$ and $\mathrm{CO}$, is governed by a charge transfer process between surface and molecule, which leads to the formation of a short-lived anionic species in the vicinity of the surface $[64,65]$. This section provides a detailed description of the underlying mechanism, forming the basis for Section 5.3.3, in which the results presented in Section 5.3.1 are discussed.

Panel (A) of Figure 5.19 show two-dimensional adiabatic potential energy surfaces (solid lines) of a hypothetical molecule-surface system. Infinitely far away from the surface, the lower potential energy surface (PES) corresponds to the neutral molecule in its ground electronic state, while the upper PES represents the respective anion. At large molecule-surface distances, the anionic state is $\Phi-E_{\mathrm{A}}$ above the neutral state, implying that the anion is formed by accepting an electron from the surface. $\Phi$ is the work function of the surface and $E_{\mathrm{A}}$ is the electron affinity of the molecule. When the molecule approaches the surface, the energy decreases due to attractive forces between the molecule and the surface. The energy of the anionic state decreases much faster than that of the neutral state because of the attractive Coulomb force between the anion and its positive image charge in the surface. In the diabatic representation (dashed lines), 
(A)

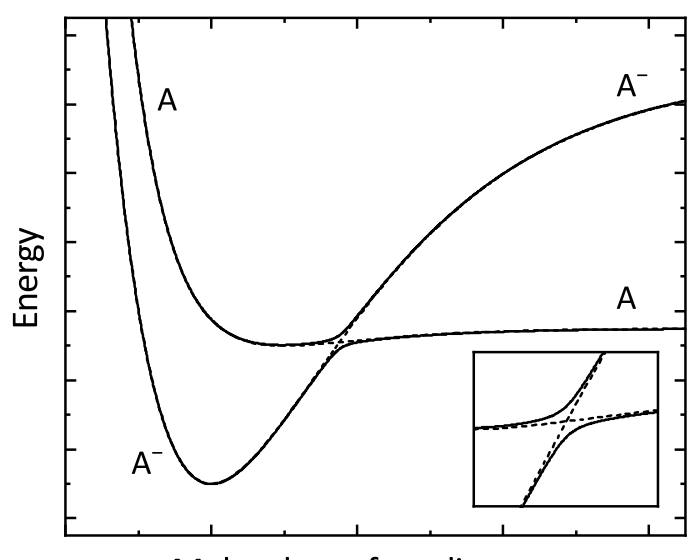

Molecule-surface distance
(B)

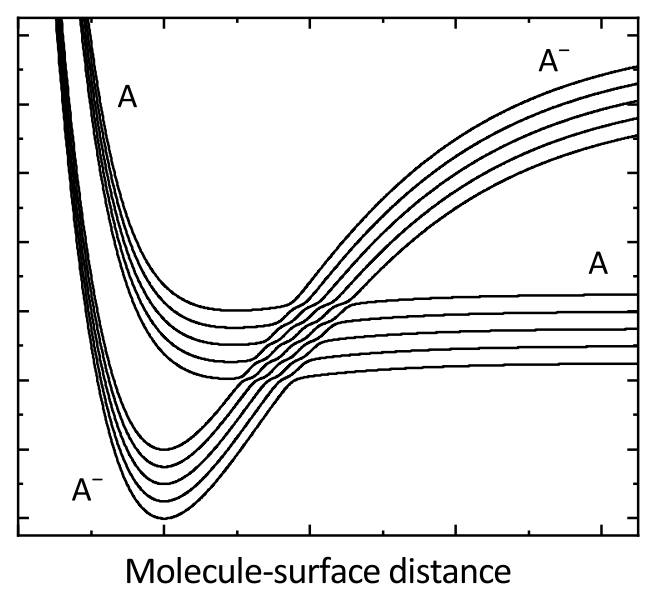

Figure 5.19: Schematic drawing of diabatic (dashed) and adiabatic (solid) twodimensional potential energy surfaces (PES) of a hypothetical moleculesurface system. Panel (A) shows the energy of the neutral molecule "A" and the respective anion " $\mathrm{A}$ " " as a function of molecule-surface distance. Following the electronically adiabatic pathway, the neutral molecule will accept an electron from the surface when it reaches the region where the diabats cross. Panel (B) shows adiabatic PESs of the same system, but includes five different electronic states of the metal. When the moleculesurface distance changes rapidly, non-adiabatic transitions between the adiabatic PES may occur in the crossing region.

the large image charge stabilization (ICS) of the anionic state results in a crossing of the anionic PES with the neutral PES at a certain molecule-surface distance. In the adiabatic representation (solid lines), however, the curve crossing is avoided, as indicated in the figure. In the electronically adiabatic picture, the molecule-surface system remains on a single adiabatic PES during the whole scattering process. Panel (A) of Figure 5.19 thus illustrates that a neutral molecule, which approaches the surface, accepts an electron from the surface and forms an anion when following the adiabatic pathway.

The electronic state of the molecule-surface system depends on both the electronic configuration of the molecule and that of the surface. The electron that is transferred from the surface to the molecule stems from a certain electronic level in the solid. Due to the high density of electronic states in the metal, the molecule-surface system is 


\section{Surface Scattering of $C O X^{1} \Sigma^{+}(v=17)$}

represented by a continuum of adiabatic PESs, as illustrated schematically in Panel (B) of Figure 5.19. In the curve crossing region, a large number of different PESs couple with each other. When the molecule-surface distance changes rapidly such that the electronic configuration of the whole system cannot follow the coordinate change adiabatically, an electronically non-adiabatic transition from one adiabatic PES to another occurs. These electronically non-adiabatic transitions play a key role in the coupling of molecular vibration to electronic degrees of freedom in the surface.

When electronically non-adiabatic transitions occur during the scattering event, the scattered molecule may leave an excited or relaxed electron-hole pair in the surface. The electronic state of the molecule, however, does not change. Instead, the molecule loses or gains vibrational energy. The coupling between large-amplitude molecular vibration and electrons in the solid is illustrated by Figure 5.20, using the benchmark system $\mathrm{NO} / \mathrm{Au}(111)$ as an example. The figure shows potential energy curves of NO (black solid) and $\mathrm{NO}^{-}$(red dot-dashed) as a function of the $\mathrm{N}-\mathrm{O}$ distance. Here, the molecule surface-distance is assumed to be $2 \AA$. The initial vibrational state is $v_{\text {inc }}=16$. Due to its different shape and equilibrium distance, the anionic state is energetically favored at the outer classical turning point of the highly vibrationally excited molecule. On the other hand, the neutral state is favored with respect to the anionic state at the inner classical turning point. In the adiabatic picture, the vibrating molecule accepts an electron from the surface at stretched geometries. The electron is transferred back to the surface as soon as the bond contracts. When, however, a non-adiabatic transition occurs, vibrational energy of the molecule can be transferred to the electron. As indicated by the blue arrows, the molecule may lose vibrational energy during one period of oscillation, creating an excited electron-hole pair.

As mentioned before, the molecule-surface distance at which the diabats shown in Panel (A) of Figure 5.19 intersect and at which the electron transfer from the surface to the molecule becomes feasible depends critically on the work function $\Phi$ of the surface and the electron affinity $E_{\mathrm{A}}$ of the molecule. With increasing $E_{\mathrm{A}}$ and decreasing $\Phi$, less ICS is needed to make the affinity level of the molecule overlap with occupied electronic states of the metal. As a consequence, the point of the curve crossing shifts to larger molecule-surface distances. On the other hand, the point shifts to shorter molecule-surface distances when $\Phi$ increases and $E_{\mathrm{A}}$ decreases. If, however, $\Phi$ is too high or $E_{\mathrm{A}}$ too low, the molecule reaches the repulsive wall and scatters off the surface before anion formation becomes possible. Thus, low $\Phi$ and high $E_{\mathrm{A}}$ favor the electron transfer process and also the non-adiabatic energy transfer between molecule and surface. Consequently, $E_{\mathrm{A}}-\Phi$ can be introduced as a parameter that classifies different molecule-surface systems by their non-adiabatic propensity $[33,34]$. The higher $E_{\mathrm{A}}-\Phi$, 


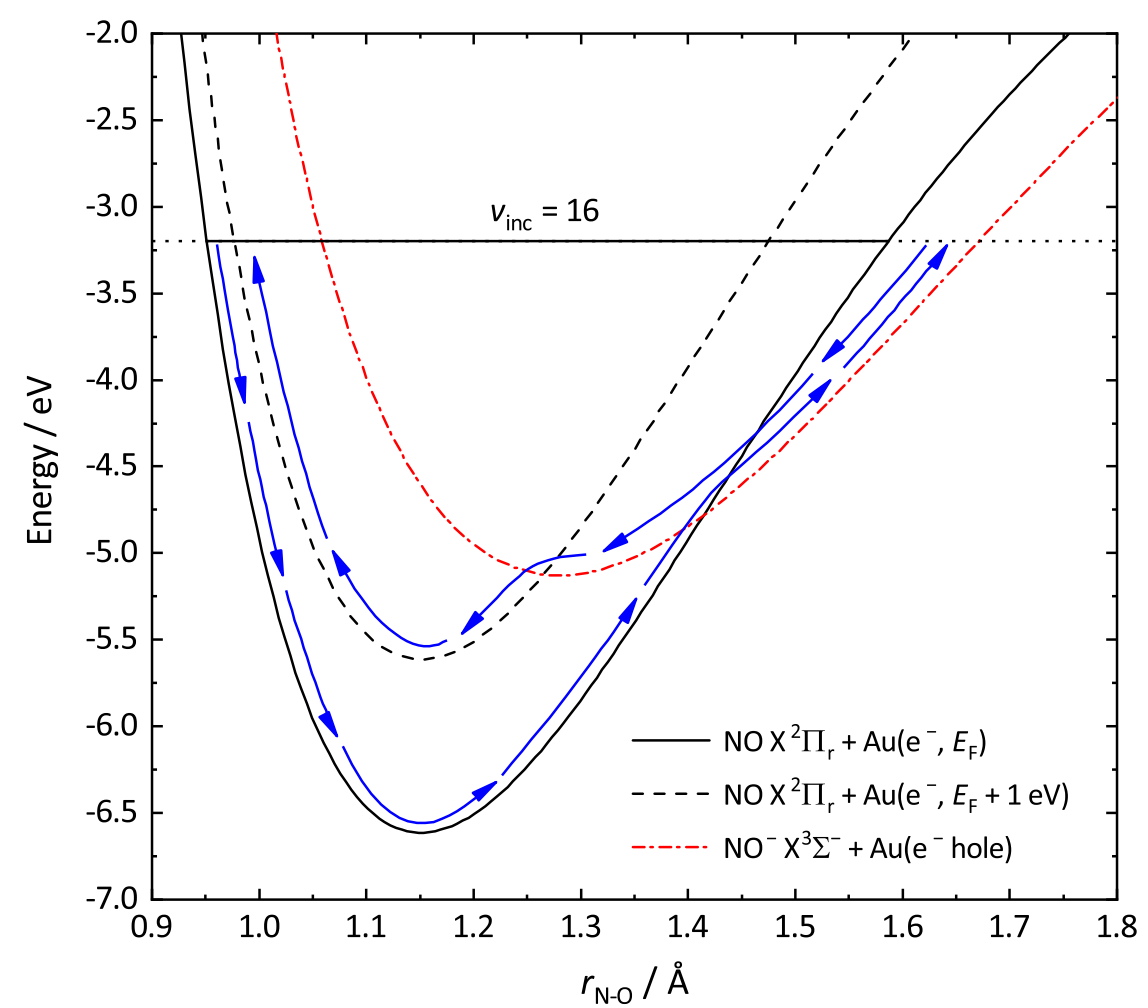

Figure 5.20: Anion mediated vibrational relaxation illustrated by potential energy curves of $\mathrm{NO} \mathrm{X} \mathrm{X}^{2} \Pi_{\mathrm{r}}$ and $\mathrm{NO}^{-} \mathrm{X}^{3} \Sigma^{-}$in the vicinity of a $\mathrm{Au}(111)$ surface. The potential energy is shown for a system consisting of a neutral NO molecule and an electron $\mathrm{e}^{-}$in the metal at the Fermi level $E_{\mathrm{F}}$ (black solid), and for neutral $\mathrm{NO}$ and an excited electron in the metal $1 \mathrm{eV}$ above $E_{\mathrm{F}}$ (black dashed). The potential energy curve of $\mathrm{NO}^{-}$(red dot-dashed) has been vertically shifted from its gas-phase position to account for the energetic penalty $\Phi$ of removing an electron from the surface and the energetic stabilization from ICS. ICS is calculated for a negative point charge $2.0 \AA$ in front of the surface. The initial vibrational state of the incident molecule is $v_{\text {inc }}=16$. The blue arrows indicate the vibrating molecule, which becomes negatively charged at stretched geometries and turns neutral again as the bond contracts. Here, $1 \mathrm{eV}$ of vibrational energy is transferred to electronic degrees of freedom of the metal, which corresponds to a vibrational energy loss of 5 quanta. Potential energy curves of $\mathrm{NO}$ and $\mathrm{NO}^{-}$are reproduced from Reference [25]. The figure is adapted from Figure 8.1. a) in Reference [129]. 


\section{Surface Scattering of $\operatorname{COX} X^{1} \Sigma^{+}(v=17)$}

the higher is the chance for a molecule to suffer anion mediated vibrational excitation or relaxation during a direct scattering event. Accordingly, Golibrzuch et al. rank the following molecule-surface systems by increasing electron-transfer propensity: $\mathrm{CH}_{4} / \mathrm{Ni}$ $<\mathrm{H}_{2} / \mathrm{Cu}<\mathrm{N}_{2} / \mathrm{Ru}<\mathrm{CO} / \mathrm{Cu}<\mathrm{HCl} / \mathrm{Au}<\mathrm{NO} / \mathrm{Au}<\mathrm{O}_{2} / \mathrm{Al}[34]$.

When vibrational relaxation from $v>0$ is considered rather than vibrational excitation from $v=0$, the situation is more complex because the electron binding energy - that is, the energy released when attaching an electron to a molecule - varies with the vibrational state of the molecule. So far, the electron affinity $E_{\mathrm{A}}$ has been considered, which is the energy difference between the vibrational ground states of the neutral molecule and the corresponding anion. Since Figure 5.20 demonstrates that the electron transfer happens preferentially at elongated bond lengths, it is more reasonable to use the vertical electron binding energy $E_{\mathrm{v}}$ at the outer classical turning point as a measure for the probability with which a molecule accepts an electron [31]. The meaning of $E_{\mathrm{A}}$ and $E_{\mathrm{v}}$ is illustrated in Panel (A) of Figure 5.21, which shows the potential energy curves of $\mathrm{NO}$ and $\mathrm{NO}^{-}$. Panel (B) shows the dependence of $E_{\mathrm{v}}$ on the internuclear distance $r_{\mathrm{N}-\mathrm{O}}$. $E_{\mathrm{v}}$ is simply calculated by subtracting the potential energy of the anion from that of the neutral species. For vibrational states discussed in this thesis $(v \leq 16$ for NO and $v \leq 17$ for $\mathrm{CO}$ ), the vertical electron binding energy at the outer classical turning point

$r_{\text {out }}$ increases with vibrational excitation. Thus, depending on shape and position of the neutral and anionic potential energy curves, the probability of the electron transfer and also the non-adiabatic propensity of a certain molecule-surface system vary with the initial vibrational state of the molecule. For $\mathrm{NO}$ and $\mathrm{CO}$, the non-adiabatic propensity increases with vibrational excitation as long as $v \leq 17$. Therefore, it is more reasonable to replace $E_{\mathrm{A}}-\Phi$ by $E_{\mathrm{v}}\left(r_{\text {out }}(v)\right)-\Phi$ in order to predict the anion-mediated vibrational relaxation probability.

Panel (A) of Figure 5.22 shows the two-dimensional adiabatic potential energy surface of the $\mathrm{NO} / \mathrm{Au}(111)$ system, providing a more detailed picture than the one-dimensional PES sketched in Figure 5.19. The effect of molecular vibration is taken into account by plotting the energy as a function of both the molecule-surface distance and the internuclear distance of $\mathrm{NO}$. The contours indicate the vibrational energies of $\mathrm{NO}(0 \leq$ $v \leq 17)$ infinitely far from the surface. The PES exhibits an attractive potential energy well that originates from the anionic character of the PES at small molecule-surface distances. The red line indicates the positions at which the adiabatic state is a mixture of $50 \%$ anionic and 50\% neutral configuration, which corresponds to the crossing of the anionic and the neutral diabats. At the crossing, the net charge of the molecule is $-0.5 e$. The shaded region marks the part of the PES at which the negative charge of the molecule is greater than $-0.5 e$. Note that the crossing region at the outer turning 
(A)

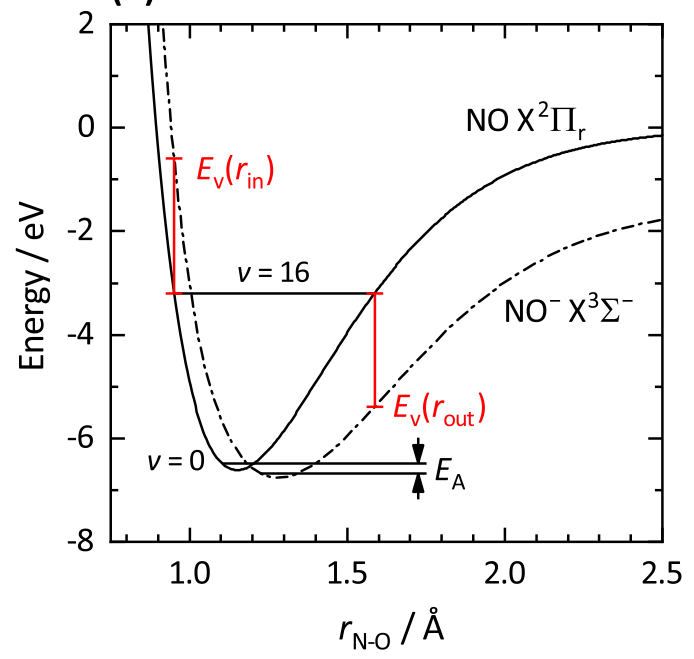

(B)

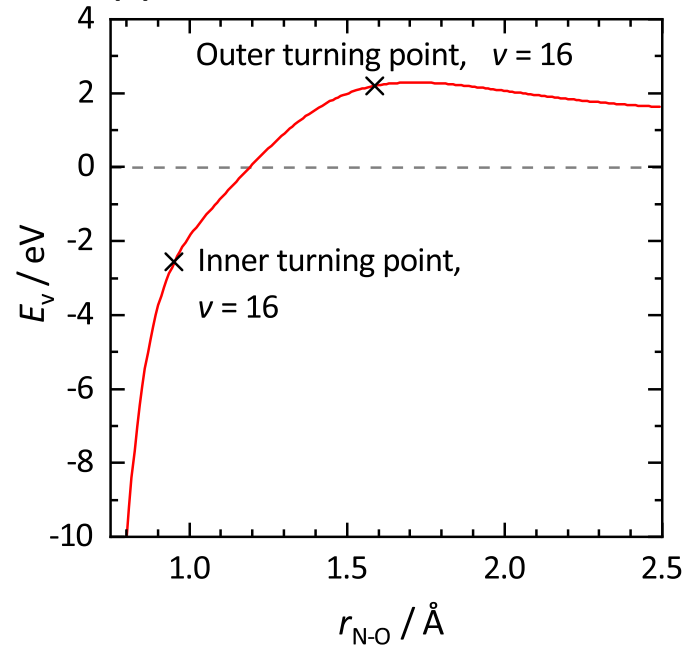

Figure 5.21: Panel (A) shows the potential energy curve of $N O X^{2} \Pi_{\mathrm{r}}$ (solid) and $\mathrm{NO}^{-}$ $\mathrm{X}^{3} \Sigma^{-}$(dot-dashed). The electron affinity $E_{\mathrm{A}}$ is shown as well as the vertical electron binding energy $E_{\mathrm{v}}$ of $\mathrm{NO}(v=16)$ at both the inner $\left(r_{\text {in }}\right)$ and the outer $\left(r_{\text {out }}\right)$ classical turning point. Panel $(\mathrm{B})$ shows the vertical electron binding energy $E_{\mathrm{v}}$ of $\mathrm{NO}$ as a function of the internuclear distance $r_{\mathrm{N}-\mathrm{O}} . E_{\mathrm{v}}$ corresponds to the difference between the $\mathrm{NO}$ and the $\mathrm{NO}^{-}$potential energy curves. The curves shown in Panel (A) and (B) are reproduced from Reference [25].

point moves to larger molecule-surface distances with increasing vibrational excitation of the molecule. The two-dimensional adiabatic PES clearly indicates that at higher vibrational excitation, the electron transfer occurs the further away from the surface. As a consequence, the probability of observing electronically non-adiabatic scattering dynamics for a certain molecule-surface system increases with vibrational excitation.

Note that the molecule-surface distance at which the anionic and neutral diabats cross should not be confused with a strict border defining the largest distance at which the electron transfer is feasible. As indicated in Panel (B) of Figure 5.22, the anionic character of the ground state adiabatic PES (red dot-dashed) rather increases gradually with decreasing surface distance. Due to the strong coupling between the diabats, anionic contributions set in $\approx 1 \AA$ before the crossing point. At the crossing point itself, the anionic contribution amounts $50 \%$.

To sum up, the vibrational relaxation (and excitation) of diatomic molecules that are 

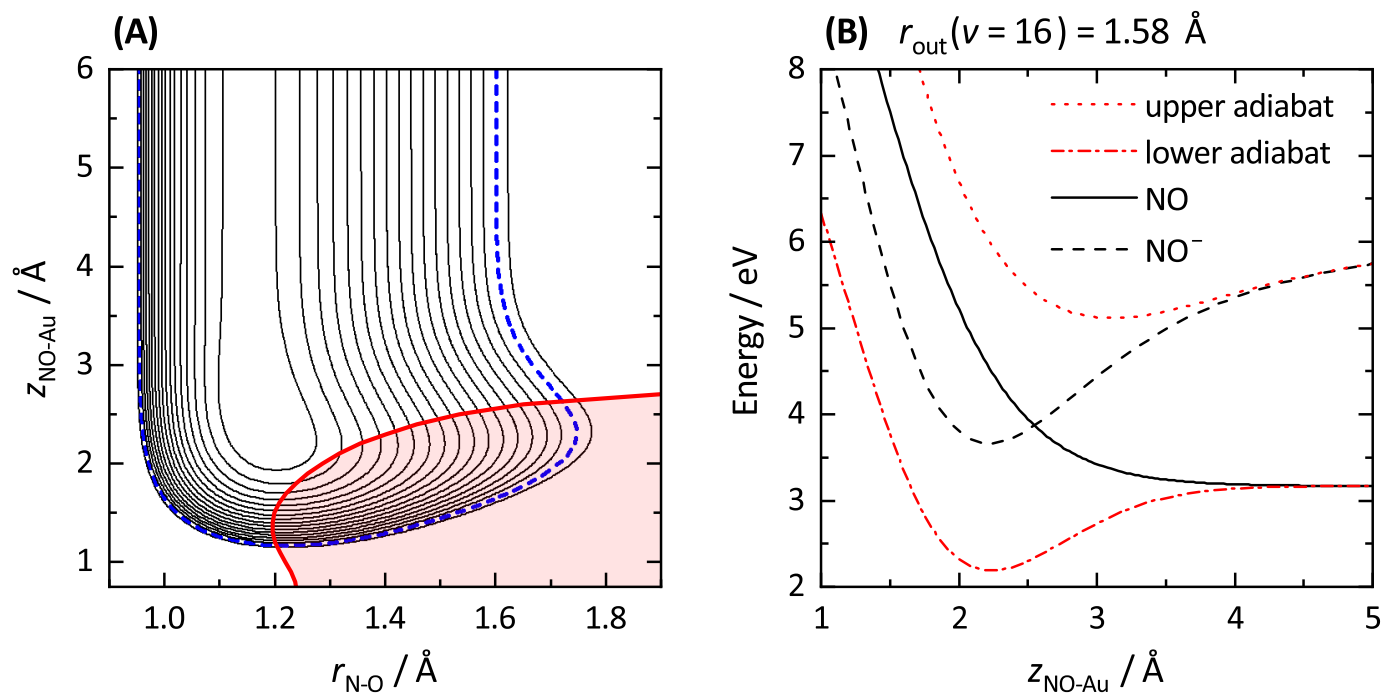

Figure 5.22: Panel (A) shows the adiabatic potential energy surface (PES) of $\mathrm{NO} / \mathrm{Au}(111)$ as a function of the molecule-surface distance $z_{\mathrm{NO}-\mathrm{Au}}$ and the internuclear distance $r_{\mathrm{N}-\mathrm{O}}$. The contours (black) indicate vibrational energies of isolated $\mathrm{NO}(0 \leq v \leq 17)$. The dashed blue contour highlights $v=16$. The red curve marks the diabatic crossing seam at which the charge of the molecule is $-0.5 e$. Note that the outer turning point crossing region moves to larger $z_{\text {NO-Au }}$ with vibrational excitation. The shaded region marks the region in which the charge is smaller than $-0.5 e$. Panel (B) shows one-dimensional diabats of $\mathrm{NO}$ (black solid) and $\mathrm{NO}^{-}$(black dashed) and the corresponding adiabatic curves (red dot-dashed and dotted). The lower adiabatic curve (red dot-dashed) corresponds to a cut through the PES shown in Panel $(\mathrm{A})$ at $r_{\mathrm{N}-\mathrm{O}}=1.58 \AA$, representing the outer classical turning point of $\mathrm{NO}(v=16)$. Due to the strong coupling, the lower adiabat exhibits anionic contributions at $z_{\mathrm{NO}-\mathrm{Au}}$ significantly larger than the crossing point of the diabats $\left(z_{\mathrm{NO}-\mathrm{Au}}=2.54 \AA\right)$.- All potential energy curves shown in Panel (A) and (B) are reproduced following the procedure described in Reference [168]. The interaction potential between $\mathrm{NO}$ and $\mathrm{Au}(111)$ is obtained by constructing a $2 \times 2$ diabatic Hamiltonian matrix based on ground-state density functional theory (DFT) calculations. The matrix consists of the neutral and anionic diagonal elements and the off-diagonal couplings. The matrix elements are fitted analytically for various $\mathrm{N}$ and $\mathrm{O}$ positions combined with an empirical $\mathrm{Au}-\mathrm{Au}$ interaction potential. 
scattered from metal surfaces can be explained by a mechanism that involves an electron transfer from the surface to the molecule. This has been demonstrated with the help of the well-known benchmark system $\mathrm{NO} / \mathrm{Au}(111)$. Basically, the mechanism comprises two steps. First, the approaching molecule accepts an electron from the surface and forms a transient anion as soon as the anionic state is sufficiently lowered in energy and overlaps with electronic states in the metal. Second, the electron is transferred back to the metal as the scattered molecule leaves the surface. Vibrational energy of the molecule is transferred to electrons in the metal if electronically non-adiabatic transitions occur during the scattering process. The amount of image charge stabilization that is needed for a certain molecule-surface system to enable the electron transfer depends on the vertical electron binding energy at the outer classical turning point of the vibrating molecule and the work function of the surface. The parameter $E_{\mathrm{v}}\left(r_{\text {out }}(v)\right)-\Phi$ has been introduced in order to classify different molecule-surface systems by their vibrational state dependent non-adiabatic propensity.

\subsubsection{Discussion: Vibrational Inelasticity}

Table 5.3 summarizes the vibrational relaxation probabilities $P_{\mathrm{R}}$ obtained for different initial conditions and compares them to results previously reported for other moleculesurface systems. Three conclusions can be drawn by comparing different combinations of $\mathrm{CO}$ and $\mathrm{NO}$ with $\mathrm{Au}(111)$ and $\mathrm{Ag}(111)$. First, NO generally exhibits higher relaxation probabilities than CO for similar $v_{\text {inc }}$. Second, the relaxation probability observed for silver is higher than that for gold. Third, the vibrational relaxation probability increases with the initial vibrational excitation of the incident molecule. In the following, it will be shown that these observations are consistent with the electron-transfer mediated relaxation mechanism introduced in Section 5.3.2.

The observed increase of $P_{\mathrm{R}}$ with the initial vibrational excitation $v_{\text {inc }}$ relies on the increase of $E_{\mathrm{v}}$ with $v_{\text {inc }}$. When $E_{\mathrm{v}}$ is higher, less ICS is required to enable the electron transfer, and the electron transfer becomes feasible farther from the surface. Panel (A) of Figure 5.23 shows $E_{\mathrm{v}}$ at the outer classical turning point $r_{\text {out }}$ as a function of the vibrational state $v$. For both $\mathrm{CO}$ (circles) and $\mathrm{NO}$ (squares), $E_{\mathrm{v}}$ increases with $v$, which agrees with the observed surface dynamics. For CO and NO, it can be concluded that electronically non-adiabatic scattering dynamics are expected to be stronger in high $v$ states than in low $v$ states.

Note that for each of the displayed $v$ states, NO exhibits a higher vertical electron binding energy than CO. This is consistent with the observation that NO has higher relaxation probabilities than $\mathrm{CO}$ at similar $v$ states. The reason for this can be found in 
5 Surface Scattering of $C O X^{1} \Sigma^{+}(v=17)$

Table 5.3: Vibrational relaxation probabilities $P_{\mathrm{R}}$ for different molecule-surface systems, initial vibrational states $v_{\text {inc }}$, and incidence translational energies $\left\langle E_{\text {inc }}\right\rangle . E_{\mathrm{v}}\left(r_{\text {out }}\left(v_{\text {inc }}\right)\right)$ is the vertical electron binding energy of the incident molecule at the classical outer turning point. $\Phi$ is the work function of the surface.

\begin{tabular}{lcccccl}
\hline \hline Molecule & Surface & $\begin{array}{c}\left\langle\boldsymbol{E}_{\text {inc }}\right\rangle \\
(\mathbf{e V})\end{array}$ & $\begin{array}{c}\boldsymbol{E}_{\mathbf{v}}\left(\boldsymbol{r}_{\text {out }}\left(\boldsymbol{v}_{\text {inc }}\right)\right) \\
(\mathbf{e V})\end{array}$ & $\begin{array}{c}\mathbf{\Phi} \\
(\mathbf{e V})\end{array}$ & $\begin{array}{c}\boldsymbol{P}_{\mathbf{R}} \\
(\boldsymbol{\%})\end{array}$ & Reference \\
\hline $\mathrm{CO}\left(v_{\text {inc }}=17\right)$ & $\mathrm{Au}(111)$ & 0.26 & 0.54 & 5.3 & 16 & this work \\
$\mathrm{CO}\left(v_{\text {inc }}=17\right)$ & $\mathrm{Au}(111)$ & 0.41 & 0.54 & 5.3 & 22 & this work \\
$\mathrm{CO}\left(v_{\text {inc }}=17\right)$ & $\mathrm{Au}(111)$ & 0.57 & 0.54 & 5.3 & 37 & this work \\
$\mathrm{CO}\left(v_{\text {inc }}=2\right)$ & $\mathrm{Au}(111)$ & 0.63 & -1.05 & 5.3 & 2 & {$[134]$} \\
\hline $\mathrm{CO}\left(v_{\text {inc }}=17\right)$ & $\mathrm{Ag}(111)$ & 0.27 & 0.54 & 4.7 & 78 & this work \\
$\mathrm{CO}\left(v_{\text {inc }}=17\right)$ & $\mathrm{Ag}(111)$ & 0.40 & 0.54 & 4.7 & 84 & this work \\
$\mathrm{CO}\left(v_{\text {inc }}=17\right)$ & $\mathrm{Ag}(111)$ & 0.57 & 0.54 & 4.7 & 85 & this work \\
$\mathrm{CO}\left(v_{\text {inc }}=2\right)$ & $\mathrm{Ag}(111)$ & 0.59 & -1.05 & 4.7 & 2 & {$[169]$} \\
\hline $\mathrm{CO}\left(v_{\text {inc }}=17\right)$ & $\mathrm{Cl} / \mathrm{Ag}(111)$ & 0.57 & 0.54 & 6.8 & 4 & this work \\
\hline $\mathrm{NO}\left(v_{\text {inc }}=16\right)$ & $\mathrm{Au}(111)$ & 0.66 & 2.20 & 5.3 & 98 & {$[86,129]$} \\
$\mathrm{NO}\left(v_{\text {inc }}=11\right)$ & $\mathrm{Au}(111)$ & 0.69 & 1.93 & 5.3 & 95 & {$[86,129]$} \\
$\mathrm{NO}\left(v_{\text {inc }}=3\right)$ & $\mathrm{Au}(111)$ & 0.65 & 0.94 & 5.3 & 57 & {$[86,129]$} \\
\hline $\mathrm{NO}\left(v_{\text {inc }}=11\right)$ & $\mathrm{Ag}(111)$ & 0.51 & 1.93 & 4.7 & 100 & {$[170]$} \\
\hline \hline
\end{tabular}

the nature of the ground state potential energy surfaces of the neutral and the anionic molecule. Panel (B) of Figure 5.23 shows the potential energy curves of neutral (solid) and anionic (dot-dashed) CO (lower panel) and NO (upper panel) as a function of the internuclear distance. Two features are striking when comparing CO with NO. First, the minimum of the $\mathrm{CO}^{-}$PES lies approximately $1.1 \mathrm{eV}$ higher in energy than that of the neutral CO PES, while the minima of the $\mathrm{NO}$ and $\mathrm{NO}^{-}$PESs are almost at the same energy. This is reflected in the electron affinity of the molecules, which is $E_{\mathrm{A}}=-1.14 \mathrm{eV}$ for $\mathrm{CO}$ [171] and $E_{\mathrm{A}}=-0.02 \mathrm{eV}$ for $\mathrm{NO}$ [172]. An increase in $E_{\mathrm{A}}$ results inevitably in an increase of $E_{\mathrm{v}}$. Second, the equilibrium bond length of $\mathrm{NO}^{-}$is significantly longer than that of neutral NO, while the equilibrium bond length of $\mathrm{CO}$ is nearly unaffected by the formation of an anion. An increased equilibrium bond length of the anionic state leads to higher $E_{\mathrm{v}}$ at the outer turning point. In conclusion, both the vertical and the horizontal shift of the anionic PES with respect to the neutral PES affect the magnitude 
(A)

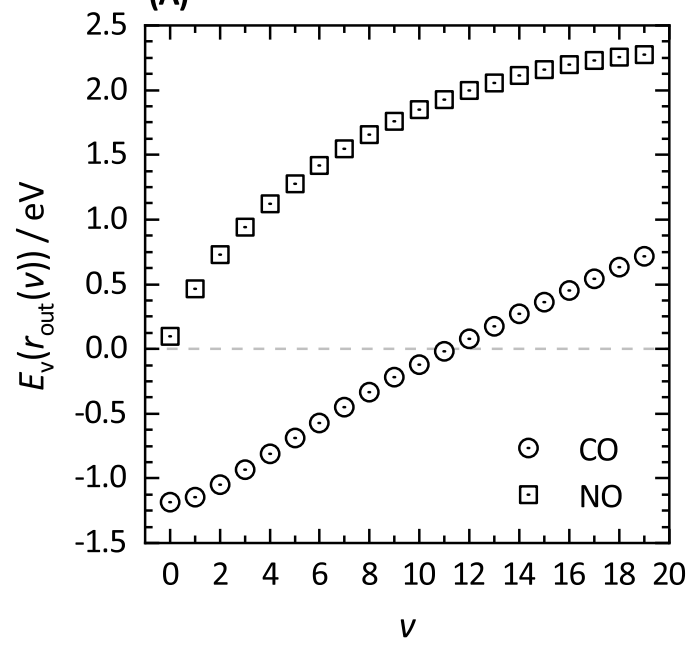

(B)

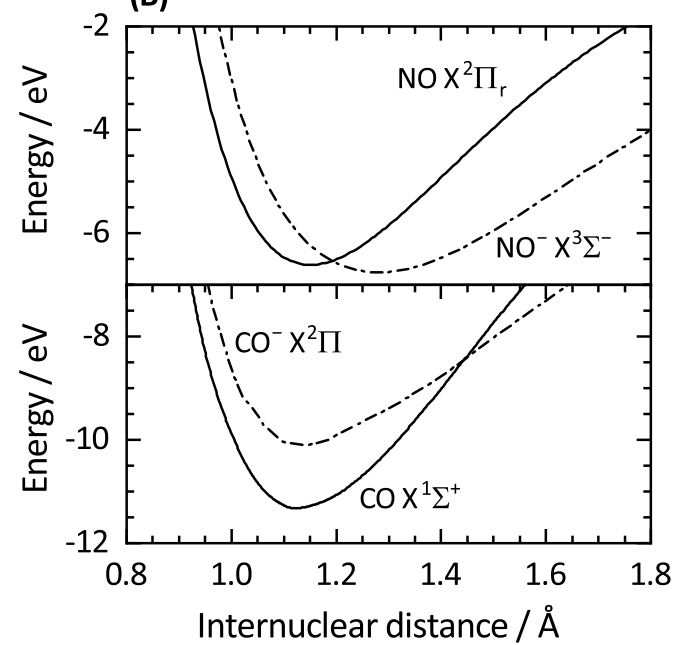

Figure 5.23: Panel (A) shows the vertical electron binding energy $E_{\mathrm{v}}$ for $\mathrm{CO}$ (circle) and NO (square) at the outer classical turning point $r_{\text {out }}$ as a function of the vibrational state $v . E_{\mathrm{v}}$ is calculated based on the gas-phase potential energy curves of neutral and anionic CO and NO shown in Panel (B). These curves are reproduced from References [171] and [25], respectively.

of $E_{\mathrm{v}}$. Furthermore, the steepness of the potential energy curves also has an influence on $E_{\mathrm{v}}$. Thus, shape and position of the electronic ground state PES of the gas-phase molecule and the respective anion provide valuable information about the dynamics at surfaces. As a first hint, the electron affinity is a useful and easily accessible parameter to evaluate the non-adiabatic propensity of a molecule-surface system. However, the change in the equilibrium bond length induced by anion formation and the resulting dependence of $E_{\mathrm{v}}$ on $v$ need to be considered when discussing non-adiabatic dynamics of vibrationally excited molecules at surfaces.

The influence of the work function $\Phi$ on $P_{\mathrm{R}}$ can be explained in a similar way as the influence of the electron binding energy. The lower $\Phi$, the earlier in the scattering process the electron transfer can take place. Consequently, $P_{\mathrm{R}}$ is higher for $\operatorname{Ag}(111)$ $(\Phi=4.7 \mathrm{eV})$ than for $\mathrm{Au}(111)(\Phi=5.3 \mathrm{eV})$, just as observed in the experiment. Note that depositing $\mathrm{Cl}$ atoms on $\mathrm{Ag}(111)$ leads to an increase of the work function by $1.5 \mathrm{eV}$ and, thus, quenches the vibrational relaxation of highly vibrationally excited CO.

The preceding discussion demonstrates that the vibrational relaxation probability depends critically on the work function of the surface and the vertical electron binding energy at the outer classical turning point of the vibrating molecule, $E_{\mathrm{v}}\left(r_{\text {out }}\left(v_{\text {inc }}\right)\right)$. 


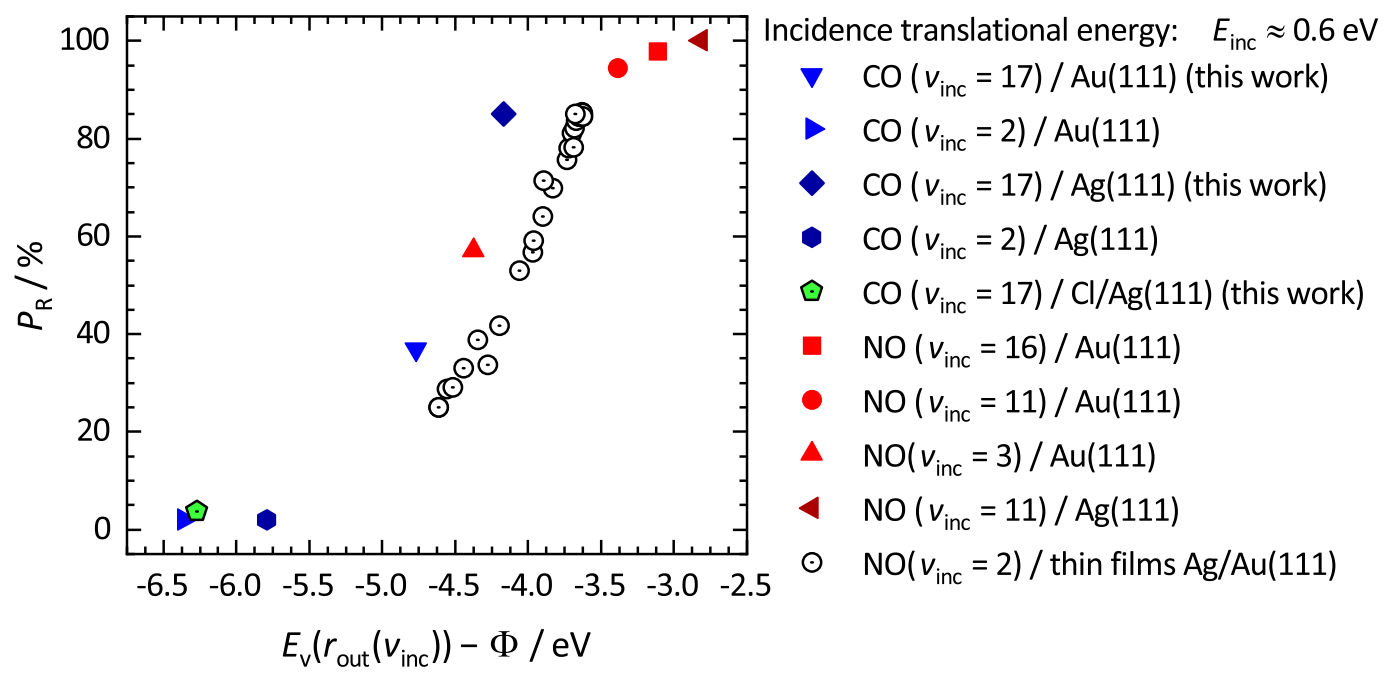

Figure 5.24: Vibrational relaxation probability $P_{\mathrm{R}}$ as a function of $E_{\mathrm{v}}\left(r_{\text {out }}\left(v_{\text {inc }}\right)\right)-\Phi$ for different molecule-surface systems $\left(E_{\mathrm{inc}} \approx 0.6 \mathrm{eV}\right)$ and different initial vibrational states. $E_{\mathrm{v}}\left(r_{\text {out }}\left(v_{\text {inc }}\right)\right)$ is the vertical electron binding energy at the outer classical turning point $r_{\text {out }}$ of a certain initial vibrational state $v_{\text {inc }} . \Phi$ is the work function of the respective surface. References for data points that do not stem from this work are listed in Table 5.3. Data points for the scattering from thin films of $\mathrm{Ag}$ on $\mathrm{Au}(111)$ are taken from Reference [169].

As proposed in Section 5.3.2, the parameter $E_{\mathrm{v}}\left(r_{\text {out }}\left(v_{\text {inc }}\right)\right)-\Phi$ serves as an indicator for the non-adiabatic propensity of a molecule-surface system and the probability of de-exciting vibrationally excited molecules. Figure 5.24 shows $P_{\mathrm{R}}$ as a function of $E_{\mathrm{v}}\left(r_{\text {out }}\left(v_{\text {inc }}\right)\right)-\Phi$ for a variety of molecule-surface systems. The figure gathers data points from several different beam-surface scattering experiments $[86,134,169,170]$. A clear correlation between $P_{\mathrm{R}}$ and $E_{\mathrm{v}}\left(r_{\text {out }}\left(v_{\text {inc }}\right)\right)-\Phi$ is noticeable. As expected, $P_{\mathrm{R}}$ increases with $E_{\mathrm{v}}\left(r_{\text {out }}\left(v_{\text {inc }}\right)\right)-\Phi$. Note that the data points describe a sigmoidal curve, which is discussed in the following.

The difference $E_{\mathrm{v}}\left(r_{\text {out }}\left(v_{\text {inc }}\right)\right)-\Phi$ is an approximate measure of the amount of ICS that is needed to lower the anionic state in energy until it crosses the neutral state, as sketched in Panel (A) of Figure 5.19. Since the image charge stabilization varies with $z^{-1}$, with $z$ being the molecule-surface distance, $E_{\mathrm{v}}\left(r_{\text {out }}\left(v_{\text {inc }}\right)\right)-\Phi$ is an indicator for the molecule-surface distance at which the neutral and the anionic diabats cross and non-adiabatic transitions are most likely to occur. If $E_{\mathrm{v}}\left(r_{\text {out }}\left(v_{\text {inc }}\right)\right)-\Phi \lesssim-6.0 \mathrm{eV}$, 
the molecules recoil from repulsive wall of the molecule-surface interaction potential before reaching the crossing region. Thus, anion formation is hampered and vibrational relaxation is very unlikely. This scenario is observed for $\operatorname{CO}\left(v_{\text {inc }}=2\right)$ on $\operatorname{Au}(111)$ and $\mathrm{Ag}(111)$ because of the small electron binding energy of $\mathrm{CO}$ in low $v$ states, and for $\mathrm{CO}\left(v_{\text {inc }}=17\right)$ on chlorinated silver because of the high work function. See Figure 5.24. If $E_{\mathrm{v}}\left(r_{\text {out }}\left(v_{\text {inc }}\right)\right)-\Phi \gtrsim-3.0 \mathrm{eV}$, the incident molecule enters the crossing region early in the scattering process. Here, anion formation is energetically feasible at moleculesurface distances that are far larger than the closest approach to the surface. These systems, such as $\mathrm{NO}\left(v_{\text {inc }}=16\right) / \operatorname{Ag}(111)$, show complete vibrational relaxation in the experiment. In the intermediate region, where $-6.0 \mathrm{eV} \lesssim E_{\mathrm{v}}\left(r_{\text {out }}\left(v_{\text {inc }}\right)\right)-\Phi \lesssim-3.0 \mathrm{eV}$, the neutral and the anionic diabats cross near the molecule-surface distance at which the molecule encounters the hard repulsive region of the potential. In this case, the vibrational relaxation probability is very sensitive to variations in $E_{\mathrm{v}}\left(r_{\text {out }}\left(v_{\text {inc }}\right)\right)$ or $\Phi$. This behavior is observed for $\mathrm{CO}$ in high $v$ states and NO in low $v$ states on $\mathrm{Au}(111)$. Switching from $\mathrm{Au}(111)$ to $\mathrm{Ag}(111)$ drastically increases the relaxation probability of $\mathrm{CO}\left(v_{\text {inc }}=17\right)$ from $37 \%$ to $85 \%$. The influence of the work function is also demonstrated by $\mathrm{NO}\left(v_{\text {inc }}=2\right)$ scattering experiments in which the work function has been smoothly varied between that of gold and silver by dosing different amounts of $\mathrm{Ag}$ atoms on $\mathrm{Au}(111)$. See Figure 5.24.

An incidence translational energy dependence of $P_{\mathrm{R}}$ is expected for molecule-surface systems that belong to the intermediate regime. For these systems, the relaxation probability is very sensitive to the closest approach to the surface. The faster the incident projectile, the closer it approaches the surface and the higher is the ICS of the anionic state. Figure 5.25 shows $P_{\mathrm{R}}$ as a function of $\left\langle E_{\text {inc }}\right\rangle$ for different moleculesurface systems. $\mathrm{NO}\left(v_{\text {inc }}=3\right) / \mathrm{Au}(111)$ and $\mathrm{CO}\left(v_{\text {inc }}=17\right) / \mathrm{Au}(111)$ show a pronounced increase of $P_{\mathrm{R}}$ with $\left\langle E_{\mathrm{inc}}\right\rangle$. The impact of $\left\langle E_{\text {inc }}\right\rangle$ is twofold. First, the ICS increases with $\left\langle E_{\text {inc }}\right\rangle$ as the molecule gets closer to the surface. Second, the velocity along the reaction coordinate increases with $\left\langle E_{\text {inc }}\right\rangle$, meaning that the probability of electronically non-adiabatic transitions also increases. The velocity dependence is less pronounced for $\mathrm{CO}\left(v_{\text {inc }}=17\right) / \operatorname{Ag}(111)$ and vanishes for $\mathrm{NO}\left(v_{\text {inc }}=16\right) / \mathrm{Au}(111)$, where the anion formation happens at large molecule-surface distances. Thus, the closest approach to the surface is no longer a critical parameter. Similarly, the velocity dependence is also almost absent for $\mathrm{CO}\left(v_{\text {inc }}=2\right) / \mathrm{Au}(111)$, for which $E_{\mathrm{v}}\left(r_{\text {out }}\left(v_{\text {inc }}\right)\right)-\Phi$ is extremely small. The repulsive wall of the molecule-surface interaction potential is so steep that the ICS gained by increasing $\left\langle E_{\text {inc }}\right\rangle$ will not suffice to compensate for the low electron binding energy of $\mathrm{CO}\left(v_{\text {inc }}=2\right)$. The molecule cannot penetrate the repulsive wall far enough to reach the curve crossing region. Consequently, the dependence of $P_{\mathrm{R}}$ on $\left\langle E_{\text {inc }}\right\rangle$ is weak. These 


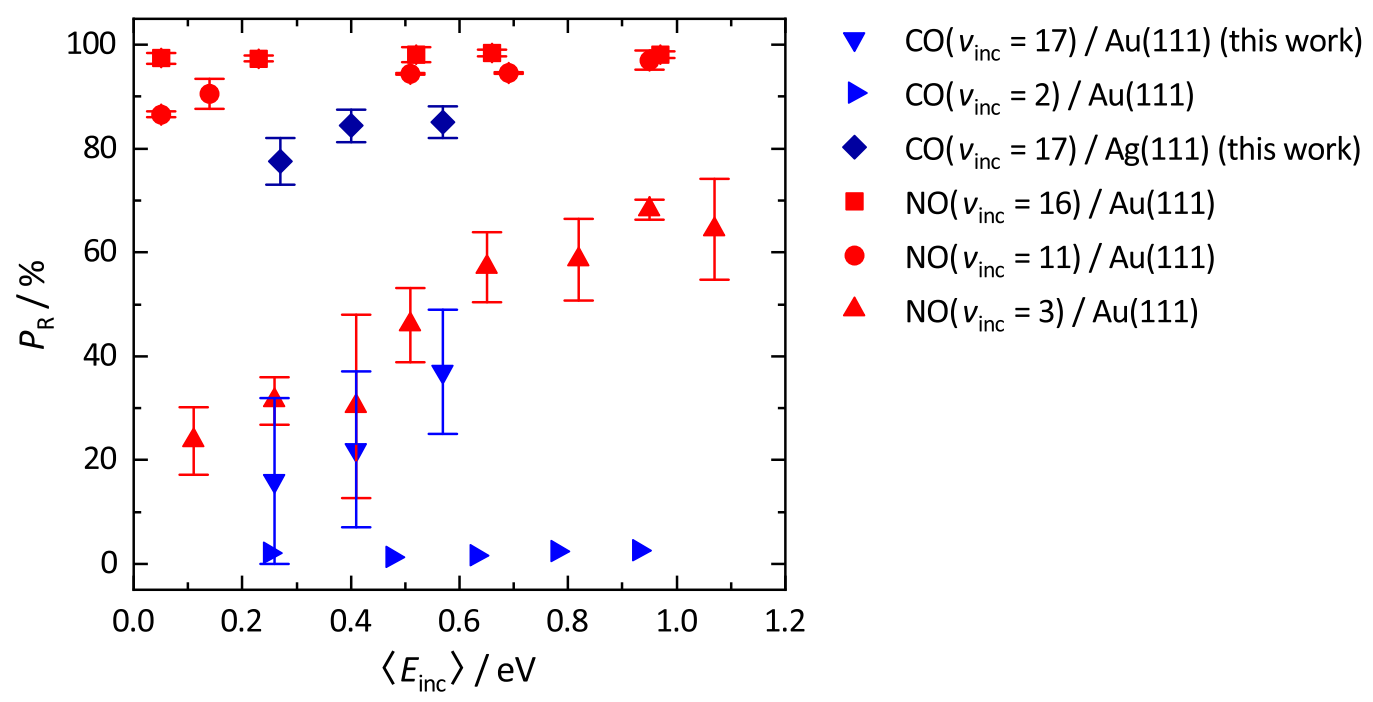

Figure 5.25: Vibrational relaxation probabilities $P_{\mathrm{R}}$ as a function of incidence translational energy $\left\langle E_{\text {inc }}\right\rangle$ for different molecule-surface systems and different initial vibrational excitations $v_{\text {inc }}$. Data for $\mathrm{CO}\left(v_{\text {inc }}=2\right) / \mathrm{Au}(111)$ and $\mathrm{NO}\left(v_{\text {inc }}=3,11,16\right) / \mathrm{Au}(111)$ are taken from Reference [134] and $[86,129]$, respectively.

features observed in the incidence translational energy dependence of $P_{\mathrm{R}}$ can be regarded as a fingerprint of electronically non-adiabatic dynamics in molecule-surface scattering and support the hypothesis of an anion mediated vibrational relaxation mechanism.

In order to estimate the position of the curve crossing, adiabatic potential energy surfaces describing the molecule-surface interaction have been calculated for different molecule-surface systems. Panel (A) of Figure 5.26 shows the adiabatic ground-state PES of the NO/Au(111) system. This PES relies on DFT calculations (GGA-PW91) that have been fitted by a $2 \times 2$ diabatic Hamiltonian, following the procedure by Roy et al. [168]. See also Figure 5.22. The Hamiltonian contains parameters representing the work function of the surface, the electron affinity of the molecule, and Morse oscillator parameters describing the vibration of the molecule. The red curve indicates the crossing of the neutral and the anionic diabats.

In order to study the effect of the work function, the PES of the NO/Ag(111) system has been approximated by only adjusting the value of the work function and leaving all other parameters unchanged [129]. See Panel (B). This approach is reasonable because the work function is the parameter that significantly changes when $\mathrm{Au}(111)$ $(\Phi=5.31 \mathrm{eV},[173])$ is replaced with $\mathrm{Ag}(111)(\Phi=4.74 \mathrm{eV},[173])$, whereas the elec- 

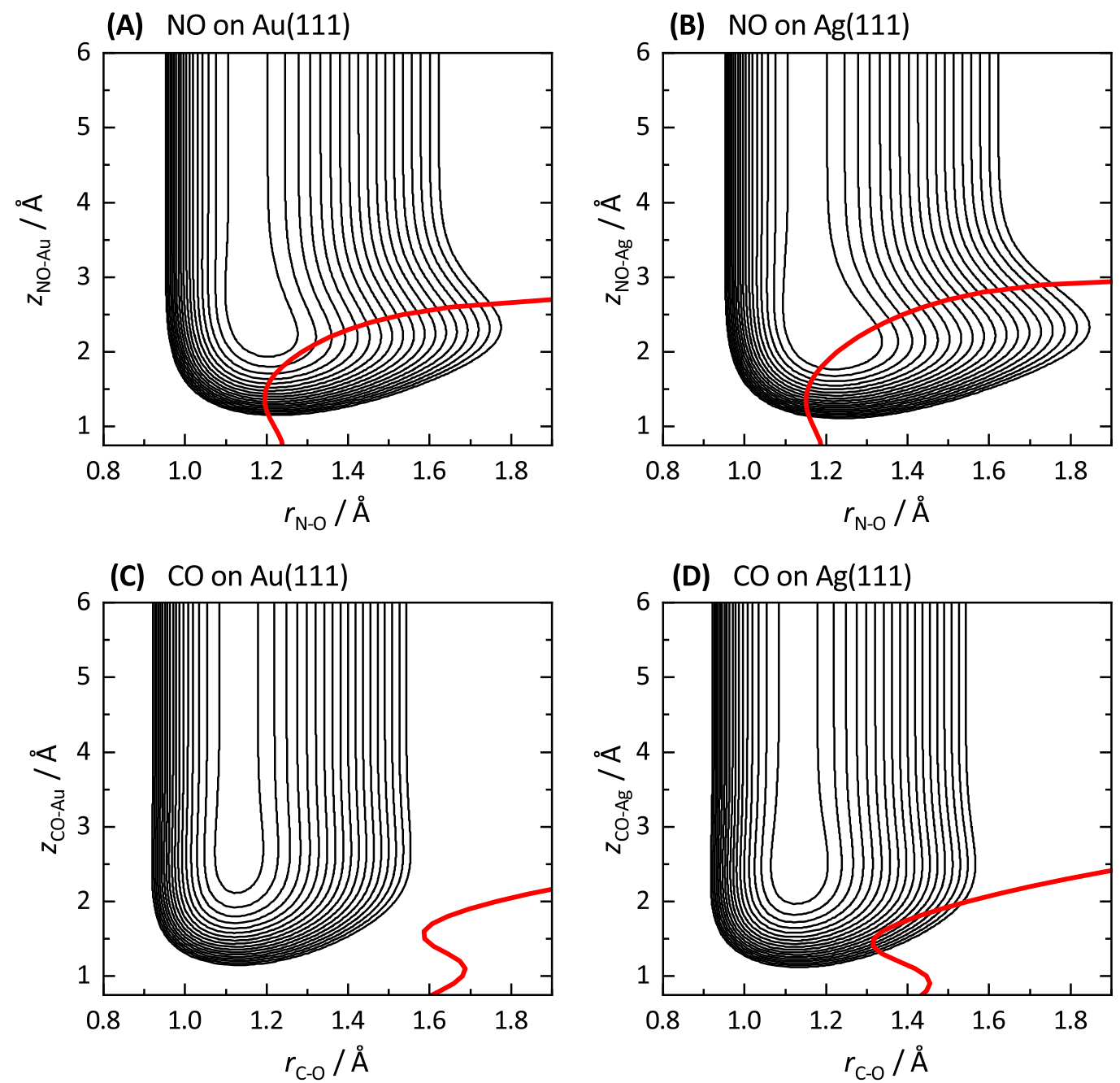

Figure 5.26: Two-dimensional adiabatic potential energy surfaces for different molecule-surface systems. The isoenergetic contours indicate vibrational energies of $0 \geq v \geq 17$. The curve crossing of the neutral and the anionic diabats is indicated by the red line, at which the net charge of the molecule is $-0.5 e$.

tronic structure [174] and the lattice constants [173] of $\mathrm{Au}(111)$ (4.0786 $\AA$ ) and $\mathrm{Ag}(111)$ $(4.0862 \AA)$ are quite similar. The comparison to $\mathrm{NO} / \mathrm{Au}(111)$ shows that the curve crossing occurs at larger molecule-surface distances for $\mathrm{NO} / \mathrm{Ag}(111)$. Thus, it is easier for NO to reach the region where non-adiabatic transitions occur, which leads to an increased vibrational relaxation probability on $\mathrm{Ag}(111)$. 


\section{Surface Scattering of $\operatorname{CO~} X^{1} \Sigma^{+}(v=17)$}

DFT data that are required to construct a PES for the $\mathrm{CO} / \mathrm{Au}(111)$ system are not available. In order to obtain an approximate PES for $\mathrm{CO} / \mathrm{Au}(111)$, the aforementioned DFT data computed for $\mathrm{NO} / \mathrm{Au}(111)$ are fitted with the only difference that the NO molecular constants are replaced by those of $\mathrm{CO}$ such that the PESs match the gas-phase PESs of $\mathrm{CO}$ and $\mathrm{CO}^{-}$at large separation from the surface. This way, electronic and vibrational properties of $\mathrm{CO}$ and $\mathrm{CO}^{-}$are adequately represented. The approach assumes that the interaction of $\mathrm{CO}$ with $\mathrm{Au}(111)$ is the same as for NO with $\mathrm{Au}(111)$. This approximation is crude since the interaction is expected to be different for open-shell NO and closed-shell CO. However, the interaction with $\mathrm{Au}(111)$ has been shown to be fairly weak in both cases. As a test, the ratio of desorption energies $E_{\text {des }}(\mathrm{CO} / \mathrm{Au}(111)): E_{\text {des }}(\mathrm{NO} / \mathrm{Au}(111))$ is extracted from the aforementioned PESs and compared to experimental results [175]. In both cases, the ratio is $0.7: 1$, demonstrating that the approximate $\mathrm{PES}$ for $\mathrm{CO} / \mathrm{Au}(111)$ makes reasonable predictions. Panel (C) in Figure 5.26 shows the crossing seam (red) for the $\mathrm{CO} / \mathrm{Au}(111)$ system. It can be seen that highly vibrationally excited $\mathrm{CO}$ cannot reach the curve crossing. Consequently, anionic contributions to the adiabatic PES are rather small near the closest surface approach. Just as $\mathrm{NO}(v=3) / \mathrm{Au}(111), \mathrm{CO}(v=17) / \mathrm{Au}(111)$ exhibits relaxation probabilities in the intermediate regime. For $\mathrm{CO}(v=2) / \mathrm{Au}(111)$, however, the anionic character of the adiabatic PES vanishes. Thus the non-adiabatic transition probability and the vibrational relaxation probability are extremely small and approach zero.

Panel (D) of Figure 5.26 displays the approximate PES as obtained for CO/Ag(111). Unlike $\mathrm{CO}$ on $\mathrm{Au}(111)$, highly vibrationally excited $\mathrm{CO}$ on $\mathrm{Ag}(111)$ reaches the crossing seam. Thus, the vibrational relaxation probability is drastically increased on $\operatorname{Ag}(111)$. For $\mathrm{NO} / \mathrm{Ag}(111)$, incident molecules traverse the crossing seam with ease, resulting in observed relaxation probabilities of $100 \%$.

In summary, vibrational relaxation probabilities and their dependence on incidence translational energy are presented for $\mathrm{CO}(v=17)$ scattered from $\mathrm{Au}(111)$ and $\mathrm{Ag}(111)$. These data complete a set of vibrational relaxation probabilities obtained from different experiments, including different molecules ( $\mathrm{CO}$ and $\mathrm{NO}$ ), different surfaces (gold and silver), and different initial vibrational excitations $\left(2 \leq v_{\text {inc }} \leq 17\right)$. The comparison of these systems clearly shows that the vibrational relaxation probability increases with the electron binding energy of the molecule and decreases with surface work function. This universal trend strongly suggests that an electron jump from the surface to the molecule lies at the essence of vibrational energy dissipation of small molecules at metal surfaces. The vibrational state dependent electron binding energy and the surface work function define the molecule-surface distance at which the neutral and the anionic diabats intersect. This is supported by potential energy surfaces that are derived from 
DFT calculations. The further away from the surface the curve crossing is located the more easily the incoming molecule can reach the curve-crossing point and the higher is the probability for electronically non-adiabatic transitions. Molecule-surface systems for which the crossing of the diabats is located near the closest approach to the surface show a pronounced velocity dependence of the relaxation probability.

\subsection{Rotational State Distributions}

When $\operatorname{CO}\left(v_{\text {inc }}=17, J_{\text {inc }}=0\right)$ is scattered from $\mathrm{Au}(111)$ and $\operatorname{Ag}(111)$, rotational excitation of the scattering products up to $J_{\text {sca }}=40$ is observed. See Figure 4.7. Final rotational state distributions are calculated from the rotationally resolved REMPI spectra shown in Figure 5.11 and 5.14. Specifically, the 7-17 and the 7-16 band are analyzed following the procedure described in Section 4.4.

Figure 5.27 shows the resultant Boltzmann plots for both vibrationally elastic $\left(v_{\text {sca }}=\right.$ 17 ) and vibrationally inelastic $\left(v_{\text {sca }}=16\right)$ scattering at $\operatorname{Au}(111)$ and $\operatorname{Ag}(111)$. The plots indicate a linear dependence on the rotational energy $E_{\mathrm{rot}}$, meaning that the rotational state distribution can be described by a Boltzmann distribution. The mean rotational energy $\left\langle E_{\text {rot }}\right\rangle$ of the scattering products can be determined from linear slopes fitted to the experimental data. Mean rotational energies and corresponding rotational temperatures are given in Tables 5.4 and 5.5. The dependence of $\left\langle E_{\text {rot }}\right\rangle$ on $\left\langle E_{\text {inc }}\right\rangle$ is plotted in Figure 5.28. Two trends should be noted. First, the rotational temperature of the scattered beam is higher than the surface temperature in all cases. Second, $\left\langle E_{\mathrm{rot}}\right\rangle$ increases linearly with $\left\langle E_{\text {inc }}\right\rangle$, indicating that the rotational excitation originates from mechanical translation-to-rotation energy transfer. Together with the narrow angular distributions presented in Section 5.2 and the linear dependence of $\left\langle E_{\text {sca }}\right\rangle$ on $\left\langle E_{\text {inc }}\right\rangle$ shown in Figure 5.4, these findings can be regarded as a fingerprint for a direct scattering mechanism.

The Boltzmann plots in Figure 5.27 do not show any evidence of orientation dependent rotational rainbow scattering [176]. For highly vibrationally excited $\mathrm{NO}\left(v_{\text {inc }}=11\right)$, however, rotational rainbows are observed when the molecules scatter vibrationally elastically from $\mathrm{Au}(111)$ [170]. The high- $J$ rainbow at around $J_{\text {sca }} \approx 35$ originates from the repulsive interaction when the molecule collides with the surface with the Oatom first. The rainbow disappears when the molecule undergoes vibrationally inelastic scattering and loses 6 vibrational quanta. Vibrational relaxation occurs preferentially for molecules that are incident with or that are dynamically steered [29] to an Natom first orientation. In the $\mathrm{N}$-atom first orientation, the rainbow is absent due to an attractive molecule-surface interaction for the $\mathrm{N}$-first orientation. The absence of 


$$
\begin{array}{ll}
\diamond\left\langle E_{\text {inc }}\right\rangle & =0.57 \mathrm{eV} \\
\square & \left\langle E_{\text {inc }}\right\rangle=0.41 \mathrm{eV} \\
\bigcirc \quad\left\langle E_{\text {inc }}\right\rangle=0.26 \mathrm{eV}
\end{array}
$$
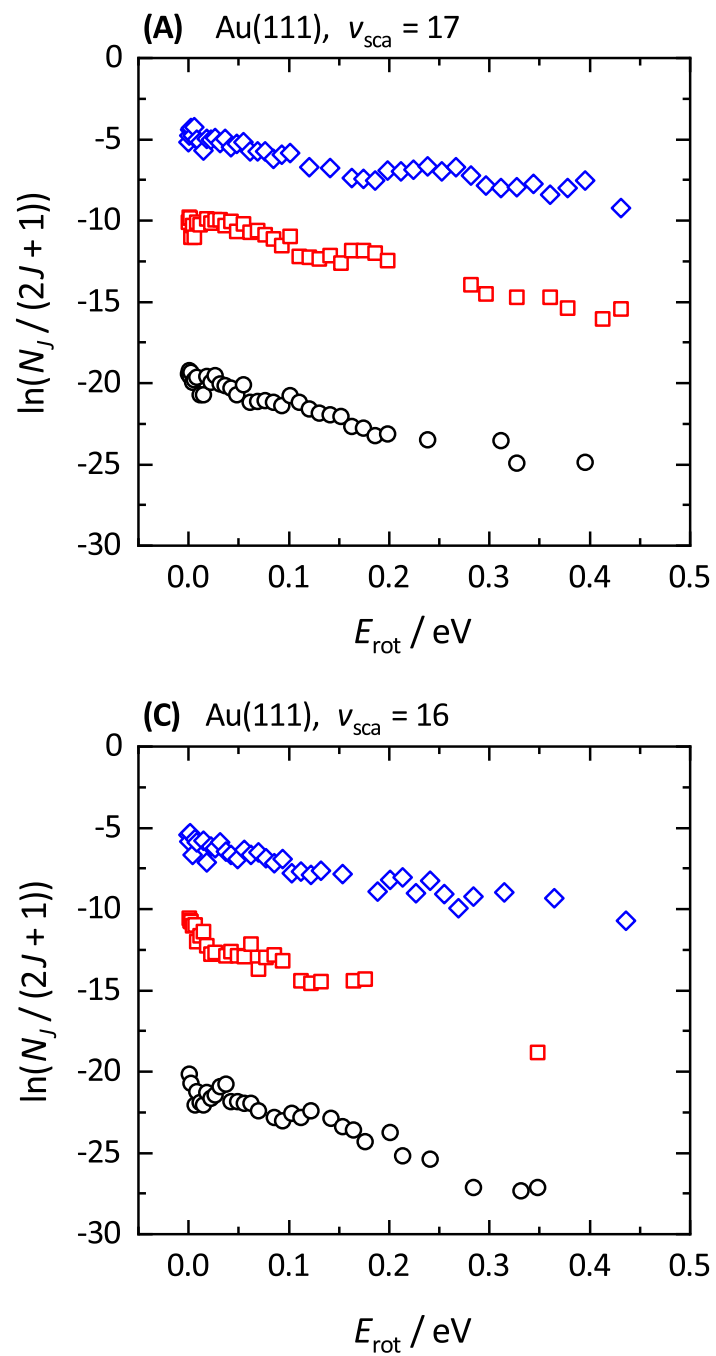

$\diamond\left\langle E_{\text {inc }}\right\rangle=0.57 \mathrm{eV}$

$\left\langle E_{\text {inc }}\right\rangle=0.40 \mathrm{eV}$

$\triangle\left\langle E_{\text {inc }}\right\rangle=0.30 \mathrm{eV}$

$\circ\left\langle E_{\text {inc }}\right\rangle=0.27 \mathrm{eV}$

(B) $\operatorname{Ag}(111), v_{\text {sca }}=17$

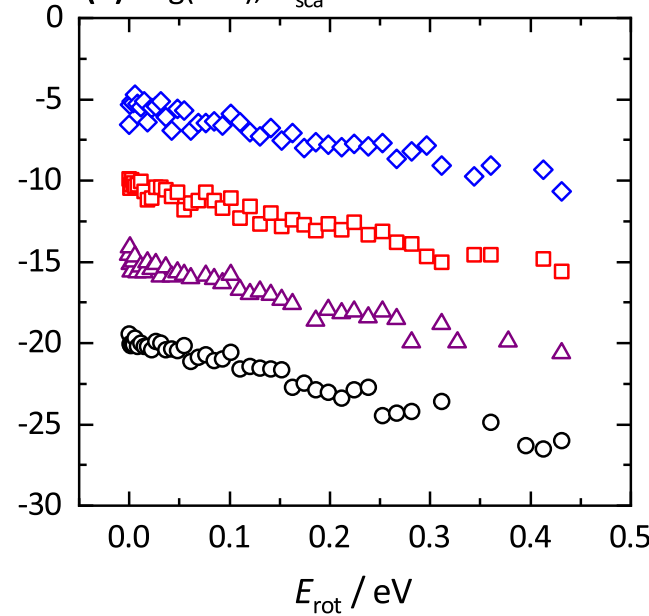

(D) $\operatorname{Ag}(111), v_{\text {sca }}=16$

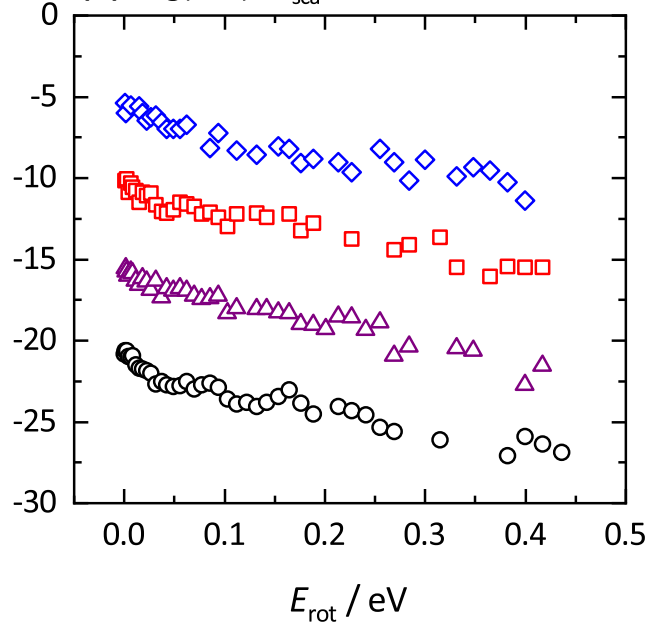

Figure 5.27: Boltzmann plots for $\mathrm{CO}\left(v_{\text {inc }}=17, J_{\text {inc }}=0 \rightarrow v_{\text {sca }}=17\right)$ scattering, Panel $(\mathrm{A})$ and $(\mathrm{B})$, and for $\mathrm{CO}\left(v_{\text {inc }}=17, J_{\text {inc }}=0 \rightarrow v_{\text {sca }}=16\right)$ scattering, Panel (C) and (D). Results are shown for the scattering from $\mathrm{Au}(111)$, Panel (A) and (C), and $\mathrm{Ag}(111)$, Panel (B) and (D), and for different incidence translational energies $\left\langle E_{\text {inc }}\right\rangle$. The vertical scales are shifted for convenience. 
Table 5.4: Mean rotational energies $\left\langle E_{\text {rot }}\right\rangle$ and rotational temperatures $T_{\text {rot }}$ for $\mathrm{CO}\left(v_{\text {inc }}=17, J_{\text {inc }}=0\right)$ scattered from $\mathrm{Au}(111)$ at different incidence translational energies $\left\langle E_{\text {inc }}\right\rangle$. Results are given for the vibrationally inelastic channel $\left(v_{\text {sca }}=17\right)$ and the vibrationally elastic channel $\left(v_{\text {sca }}=16\right) .\left\langle E_{\text {rot }}\right\rangle$ and $T_{\text {rot }}$ are determined from Boltzmann plots shown in Figure 5.27. Note that values in parentheses are not representative because the REMPI signal was too weak to detect high $J_{\text {sca }}$ (see red trace in Panel (C) of Figure 5.27).

\begin{tabular}{|c|c|c|c|c|}
\hline \multicolumn{5}{|c|}{$\overline{\mathrm{Au}(111)}$} \\
\hline \multirow[b]{2}{*}{$\left\langle\boldsymbol{E}_{\text {inc }}\right\rangle / \mathrm{eV}$} & \multicolumn{2}{|c|}{$v_{\text {sca }}=17$} & \multicolumn{2}{|c|}{$v_{\mathrm{sca}}=16$} \\
\hline & $\left\langle E_{\text {rot }}\right\rangle / \mathrm{meV}$ & $T_{\text {rot }} / \mathbf{K}$ & $\left\langle\boldsymbol{E}_{\text {rot }}\right\rangle / \mathrm{meV}$ & $T_{\text {rot }} / \mathbf{K}$ \\
\hline 0.57 & 100 & 1170 & 83 & 970 \\
\hline 0.41 & 80 & 930 & $(30)$ & $(320)$ \\
\hline 0.26 & 55 & 640 & 50 & 580 \\
\hline
\end{tabular}

Table 5.5: Mean rotational energies $\left\langle E_{\text {rot }}\right\rangle$ and rotational temperatures $T_{\text {rot }}$ for $\mathrm{CO}\left(v_{\text {inc }}=17, J_{\text {inc }}=0\right)$ scattered from $\operatorname{Ag}(111)$ at different incidence translational energies $\left\langle E_{\text {inc }}\right\rangle$. Results are given for the vibrationally inelastic channel $\left(v_{\mathrm{sca}}=17\right)$ and the vibrationally elastic channel $\left(v_{\mathrm{sca}}=16\right) .\left\langle E_{\mathrm{rot}}\right\rangle$ and $T_{\text {rot }}$ are determined from Boltzmann plots shown in Figure 5.27.

\begin{tabular}{|c|c|c|c|c|}
\hline \multicolumn{5}{|c|}{$\operatorname{Ag}(111)$} \\
\hline \multirow[b]{2}{*}{$\left\langle E_{\text {inc }}\right\rangle / \mathrm{eV}$} & \multicolumn{2}{|c|}{$v_{\mathrm{sca}}=17$} & \multicolumn{2}{|c|}{$v_{\text {sca }}=16$} \\
\hline & $\left\langle E_{\text {rot }}\right\rangle / \mathrm{meV}$ & $T_{\text {rot }} / \mathbf{K}$ & $\left\langle\boldsymbol{E}_{\mathrm{rot}}\right\rangle / \mathrm{meV}$ & $T_{\text {rot }} / \mathrm{K}$ \\
\hline 0.57 & 89 & 1030 & 74 & 860 \\
\hline 0.40 & 74 & 860 & 87 & 1010 \\
\hline 0.30 & 69 & 800 & 71 & 830 \\
\hline 0.27 & 67 & 780 & 64 & 740 \\
\hline
\end{tabular}

a high- $J$ rainbow for vibrationally elastically scattered $\mathrm{CO}\left(v_{\text {inc }}=17\right)$ suggests that orientation dependent effects are less important for high- $v$ CO than for high- $v$ NO. However, it remains unclear why high- $J$ rainbows are absent in $\mathrm{CO}\left(v_{\text {inc }}=17\right) / \mathrm{Au}(111)$ scattering, while strong rainbows are reported for $\mathrm{CO}\left(v_{\text {inc }}=0\right) / \mathrm{Au}(111)$ scattering [75]. 
5 Surface Scattering of $C O X^{1} \Sigma^{+}(v=17)$

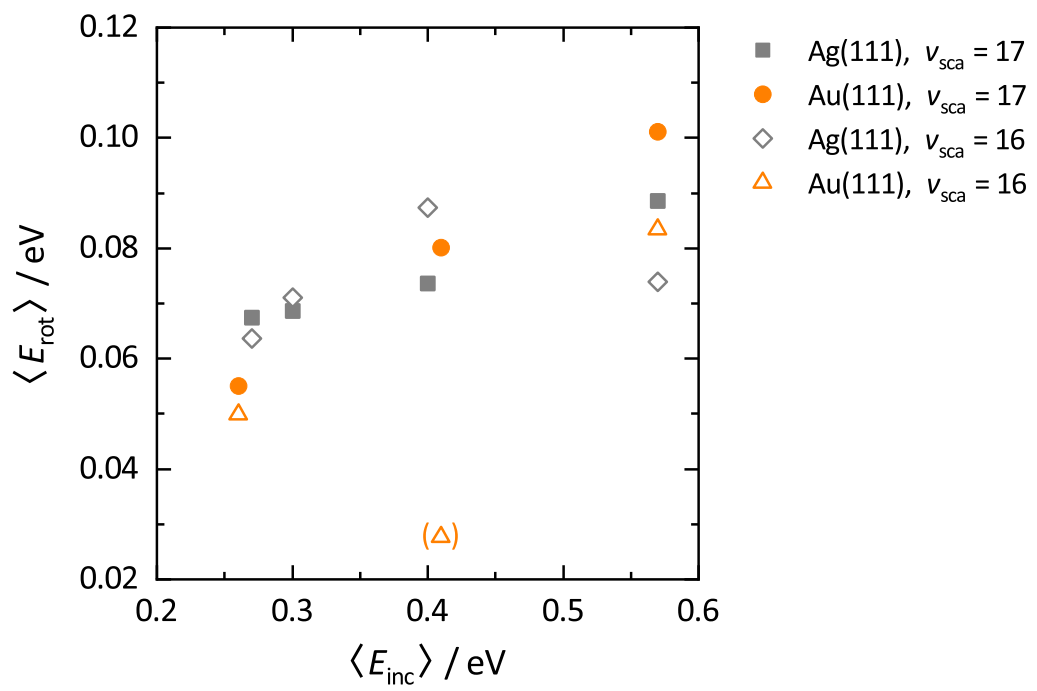

Figure 5.28: Mean rotational energy $\left\langle E_{\text {rot }}\right\rangle$ of surface scattered $\operatorname{CO}\left(v_{\text {inc }}=17, J_{\text {inc }}=0\right)$ as a function of incidence translational energy $\left\langle E_{\text {inc }}\right\rangle$. Note that the data point for vibrationally inelastic scattering $\left(v_{\text {sca }}=16\right)$ from $\mathrm{Au}(111)$ at $\left\langle E_{\text {inc }}\right\rangle=0.41 \mathrm{eV}$ is not representative because the REMPI signal was too weak to detect high $J_{\text {sca }}$ (see red trace in Panel (C) of Figure 5.27). 


\section{Towards Stark-Decelerated Beams of CO $\mathrm{X}^{1} \Sigma^{+}(v=17)$}

\subsection{Motivation}

Scattering experiments in which a molecular beam of highly vibrationally excited NO collides with a low-work function cesium-dosed $\mathrm{Au}(111)$ surface demonstrate the importance of electronic non-adiabaticity in molecule-surface dynamics [45, 88, 91]. As the vibrational energy of $\mathrm{NO}\left(v_{\mathrm{inc}}=18\right)$, which is $E_{\mathrm{vib}}=3.4 \mathrm{eV}$, exceeds the work function of $\mathrm{Cs} / \mathrm{Au}(111)$, which is $\Phi=1.6 \mathrm{eV}$ [90], electron emission from the surface is observed when the vibration is quenched $[92,93]$. The direct detection of ejected electrons provides strong evidence for the fact that the nuclear motion of a large-amplitude vibration can induce electronic excitation, a process that violates the Born-Oppenheimer approximation [89]. Interestingly, the electron yield increases with decreasing incidence translational energy $\left\langle E_{\text {inc }}\right\rangle[177]$. This inverse velocity dependence can be understood in terms of the window of opportunity model which defines a distinct region in front of the surface where electron emission is energetically feasible. One edge of the window is determined by the work function of the surface and the electron binding energy of the molecule, defining the largest molecule-surface distance at which an electron can be transferred from the surface to the molecule. Due to non-adiabatic transitions, the molecule may lose enough vibrational energy to elevate a surface electron above the vacuum level. The other edge of the window originates from the extremely low work function, which leads to an energetic situation where - at short distances - the anion is energetically favored to such an extend that it is more stable than the neutral regardless of whether the NO bond is stretched or compressed. As a result, electron emission is suppressed in this region. The existence of the second edge makes the scattering dynamics of high- $v$ diatomic molecules at low-work function surfaces different form those at $\mathrm{Au}(111)$ with $\Phi=5.31 \mathrm{eV}$ or $\mathrm{Ag}(111)$ with $\Phi=4.74 \mathrm{eV}$.

For $\mathrm{NO}\left(v_{\text {inc }}=18\right)$ scattering at cesium-covered $\mathrm{Au}(111)$, the electron yield increases from $3 \%$ to $13 \%$ when the molecules are slowed down from $v=2500 \mathrm{~m} / \mathrm{s}\left(E_{\mathrm{inc}}=0.97 \mathrm{eV}\right)$ to $430 \mathrm{~m} / \mathrm{s}\left(E_{\mathrm{inc}}=0.03 \mathrm{eV}\right)$. As the electron yield tends to increase linearly with $v^{-1}$, a 
significantly higher electron emission efficiency is conceivable for even slower molecular beams. The Stark decelerator used in the present work is capable of decelerating a supersonic molecular beam of metastable CO from $360 \mathrm{~m} / \mathrm{s}\left(E_{\text {inc }}=0.02 \mathrm{eV}\right)$ to $100 \mathrm{~m} / \mathrm{s}$ $\left(E_{\text {inc }}=0.002 \mathrm{eV}\right)$, covering a velocity range that is not accessible with conventional molecular beam techniques such as supersonic jet expansion. Thus, employing Starkmanipulated molecular beams in state-of-the-art beam-surface scattering setups appears to be an ideal choice for studying the role of electronically non-adiabatic dynamics in the interaction of highly vibrationally excited molecules with low-work function surfaces. This chapter summarizes the attempts that have been made to prepare and scatter Stark-decelerated molecular beams of $\mathrm{CO}\left(v_{\text {inc }}=17\right)$. Nevertheless, the planned surfacescattering experiment could not be accomplished because the molecular beam intensity produced by the decelerator was too low to apply the required laser preparation and detection schemes in a practicable way.

\subsection{Approaches}

\subsubsection{Velocity Scans}

Prior to any scattering experiment, the velocity with which the molecular beam leaves the decelerator is adjusted manually by loading the respective high-voltage triggers in KouDA - the computer program that controls the delay generator. However, this procedure is time consuming and impractical if the experiment needs to be repeated for many different velocities. Thus, a procedure has been developed that allows automated velocity scans, exploiting the full advantages of the Stark decelerator as a beam-manipulation tool.

Forty burst files - the files that contain the timings of the high-voltage triggers - have been generated for final velocities between $24 \mathrm{~m} / \mathrm{s}\left(\phi_{0}=79^{\circ}\right)$ and $512 \mathrm{~m} / \mathrm{s}\left(\phi_{0}=-90^{\circ}\right)$ using the libcoldmol software [137]. Interpolation of the high-voltage triggers allows the generation of burst files for any desired velocity between $24 \mathrm{~m} / \mathrm{s}$ and $512 \mathrm{~m} / \mathrm{s}$ without solving the equations of motion again. By defining a start velocity, an end velocity, and the step size of the velocity scan, an extended burst file can be generated which is composed of burst segments, each of which consists of a specific burst file representing a certain velocity. When feeding KouDA with the extended burst file, a new triggering sequence can be loaded automatically after each cycle of experiment by switching the segment that is read. In this manner, the velocity can be switched after each cycle of experiment, enabling velocity scans in which beam-surface scattering experiments with different beam velocities can be conducted with a $10 \mathrm{~Hz}$ repetition rate. 
(A)

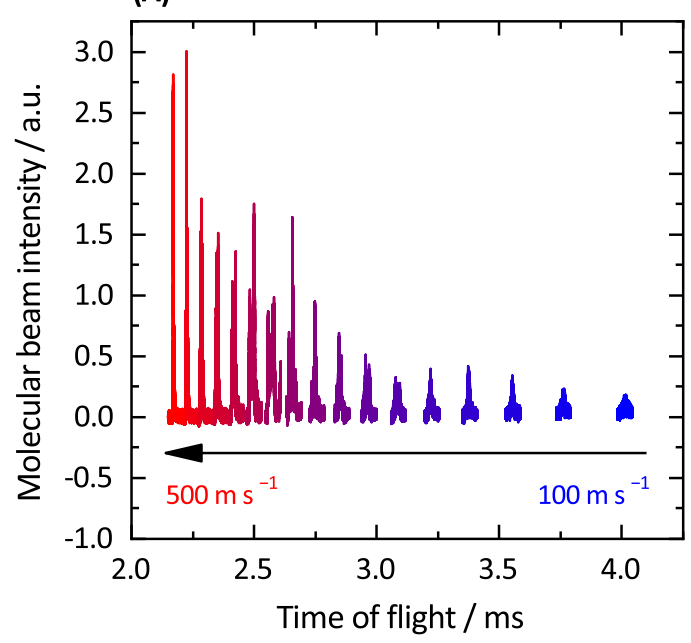

(B)

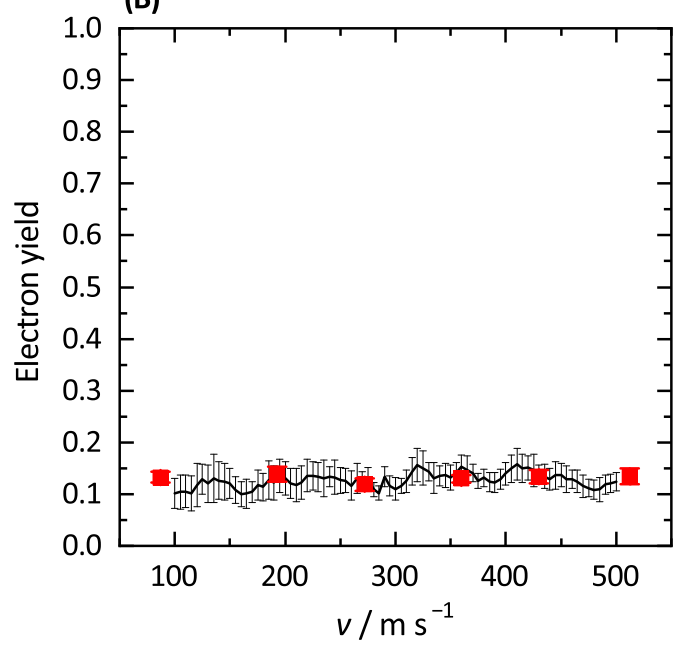

Figure 6.1: Panel (A) shows molecular beam profiles as a function of flight time for different beam velocities. Beam profiles are measured by collecting electrons emitted from $\mathrm{Au}(111)$ due to metastable $\mathrm{CO}$ quenching. The $x$-axis indicates the flight time from the point at which metastable $\mathrm{CO}$ is prepared by laser excitation to the surface. Panel (B) shows the electron yield measured for the de-excitation of metastable $\mathrm{CO}$ at $\mathrm{Au}(111)$ as a function of the velocity $v$ of the incoming beam. Results obtained by performing a velocity scan (black) are compared to results obtained by manual adjustment of the velocity (red) [178].

Panel (A) of Figure 6.1 shows molecular beam profiles for different velocities. The beam profile is monitored by collecting the electrons emitted from $\mathrm{Au}(111)$ as soon as metastable CO collides with the surface and gets de-excited. The horizontal axis indicates the flight time of the molecular beam. Each beam profile has been measured separately by repeating the experiment using different segments of a burst file that was designed for velocity scans between $100 \mathrm{~m} / \mathrm{s}$ and $500 \mathrm{~m} / \mathrm{s}$. Panel (A) illustrates that the velocity of a molecular beam incident on a surface can be easily tuned to values between $100 \mathrm{~m} / \mathrm{s}$ and $500 \mathrm{~m} / \mathrm{s}$. Note that the signal is extremely weak for velocities near or below $100 \mathrm{~m} / \mathrm{s}$ as the amount of metastable CO in the beam decreases with increasing flight time. The radiative lifetime of metastable $\mathrm{CO}$ is $2.63 \mathrm{~ms}$ [112].

For an incident beam velocity of $360 \mathrm{~m} / \mathrm{s}$, the absolute electron yield $\gamma$ observed for the quenching of metastable $\mathrm{CO}$ at $\mathrm{Au}(111)$ is 0.13 [179]. Panel (B) of Figure 6.1 shows $\gamma$ as a function of incidence velocity. The figure compares results obtained from 
a velocity scan (black), conducted from $100 \mathrm{~m} / \mathrm{s}$ to $500 \mathrm{~m} / \mathrm{s}$ with steps of $5 \mathrm{~m} / \mathrm{s}$, with results obtained by manual adjustment of the beam velocity (red) [178]. The results obtained by the two methods are consistent. In both cases, no velocity dependence of $\gamma$ is observed. The larger error bars of the black trace are due to a smaller number of averages.

In summary, the Stark machine has been improved by setting up automated velocity scans, which serves as an important tool in velocity-controlled beam-surface scattering experiments.

\subsubsection{Preparation of Stark-decelerated $\mathrm{CO} \mathrm{X}^{1} \Sigma^{+}(v=17)$}

$\Sigma$ states of diatomic molecules with a permanent electric dipole moment do not exhibit a first order Stark splitting because the dipole moment is perpendicular to the total angular momentum and the resultant electric dipole moment along the electric field lines is zero. Thus, molecular beams of $\mathrm{CO}$ in the ground electronic state $\mathrm{X}^{1} \Sigma^{+}$ cannot be Stark-decelerated. In order to Stark-decelerate a $\mathrm{CO}$ beam, the molecules are excited to the metastable $\mathrm{a}^{3} \Pi_{1}$ state, which has a sufficiently large component of the dipole moment parallel to the total angular momentum. Thus, the production of Stark-decelerated $\mathrm{CO} \mathrm{X}{ }^{1} \Sigma^{+}$requires laser preparation schemes that allow the transfer of metastable $\mathrm{CO}$ back to the ground electronic state after the beam has exited the decelerator. In order to produce $\mathrm{CO} \mathrm{X}^{1} \Sigma^{+}$in high $v$ states, the pump-pump-perturbdump $\left(\mathrm{P}^{3} \mathrm{D}\right)$ scheme is employed [106]. The first pump laser excites the $\mathrm{a}^{3} \Pi \leftarrow \mathrm{X}^{1} \Sigma^{+}$ transition prior to Stark deceleration. After having passed through the decelerator, metastable CO is transferred back to the $\mathrm{X}^{1} \Sigma^{+}$manifold by using a second pump laser and a dump laser. Laser frequencies are chosen such that $\mathrm{CO}^{1} \Sigma^{+}(v=17)$ is produced. See Section 3.4.1.

Figure 6.2 demonstrates that the $\mathrm{P}^{3} \mathrm{D}$ optical pumping scheme has been successfully applied to the production of highly vibrationally excited CO starting from metastable $\mathrm{CO}$ that has left the decelerator. The figure shows $(1+1)$ REMPI spectra probing the presence of $\mathrm{CO} \mathrm{X}^{1} \Sigma^{+}(v=17, J=0,2)$ in the molecular beam. The temporal beam profile (blue) of the $\mathrm{CO} \mathrm{X}^{1} \Sigma^{+}(v=17)$ beam is presented in Figure 6.3. Note that the high- $v$ CO beam has been prepared by laser exciting the most intense part of the $\mathrm{CO}$ $\mathrm{a}^{3} \Pi_{1}$ beam (black). The ion MCP intensities represent typical intensities that can be achieved with the experimental apparatus.

Even though Stark-decelerated $\mathrm{CO} \mathrm{a}^{3} \Pi_{1}$ is successfully converted to $\mathrm{CO} \mathrm{X}^{1} \Sigma^{+}$, the amount of $\mathrm{CO} \mathrm{X}^{1} \Sigma^{+}$in the beam is extremely low, which results in REMPI intensities at the limit of detection. See for instance Figure 6.2, in which transitions probing states 
(A)



(B)



Figure 6.2: $(1+1)$ REMPI spectra via $\mathrm{A}^{1} \Pi\left(v^{\prime}=8\right) \leftarrow \mathrm{X}^{1} \Sigma^{+}\left(v^{\prime \prime}=17\right)$ of highly vibrationally excited $\mathrm{CO}$ prepared by means of $\mathrm{P}^{3} \mathrm{D}$. Spectra are recorded for the dump laser turned on (red) and turned off (black). By tuning the dump laser's frequency, population can be transferred selectively either into $\mathrm{X}^{1} \Sigma^{+}\left(v^{\prime \prime}=17, J^{\prime \prime}=0\right)$, Panel $(\mathrm{A})$, or into $\mathrm{X}^{1} \Sigma^{+}\left(v^{\prime \prime}=17, J^{\prime \prime}=2\right)$, Panel (B). The molecular beam is guided through the decelerator $\left(\phi_{0}=0^{\circ}\right.$, $s=3)$.

prepared by Franck-Condon pumping (dump off) hardly emerge from the background. See also Figure 6.3 in which the $\mathrm{CO} \mathrm{X}^{1} \Sigma^{+}$time-of-flight trace exhibits a high level of noise. The difficulties in the detection of Stark-decelerated $\mathrm{CO} \mathrm{X}{ }^{1} \Sigma^{+}$are also illustrated by Figure 6.4, showing a REMPI scan in which both highly vibrationally excited CO and residual metastable $\mathrm{CO}$ in the molecular beam are probed at the same time. Since the resonant steps in the REMPI schemes are strong transitions in both cases, the weak signal obtained for $\mathrm{CO} \mathrm{X}^{1} \Sigma^{+}(v=17)$ is due to the small amount of $\mathrm{CO} \mathrm{X}^{1} \Sigma^{+}(v=17)$ in the beam. This has several reasons. First, the total number of molecules in the Starkmanipulated beam is reduced with respect to conventional supersonic beams because the Stark decelerator cuts out a small part of the initial phase-space distribution. However, this might lead to rather small losses as the most intense part of the distribution is guided with high efficiency through the decelerator. Note that beam manipulation by pulsed lasers is also capable of exciting only the most intense part of the initial distribution. The losses of the decelerator may increase though when the decelerator is operated in deceleration or acceleration mode rather than guiding mode. Second, the 


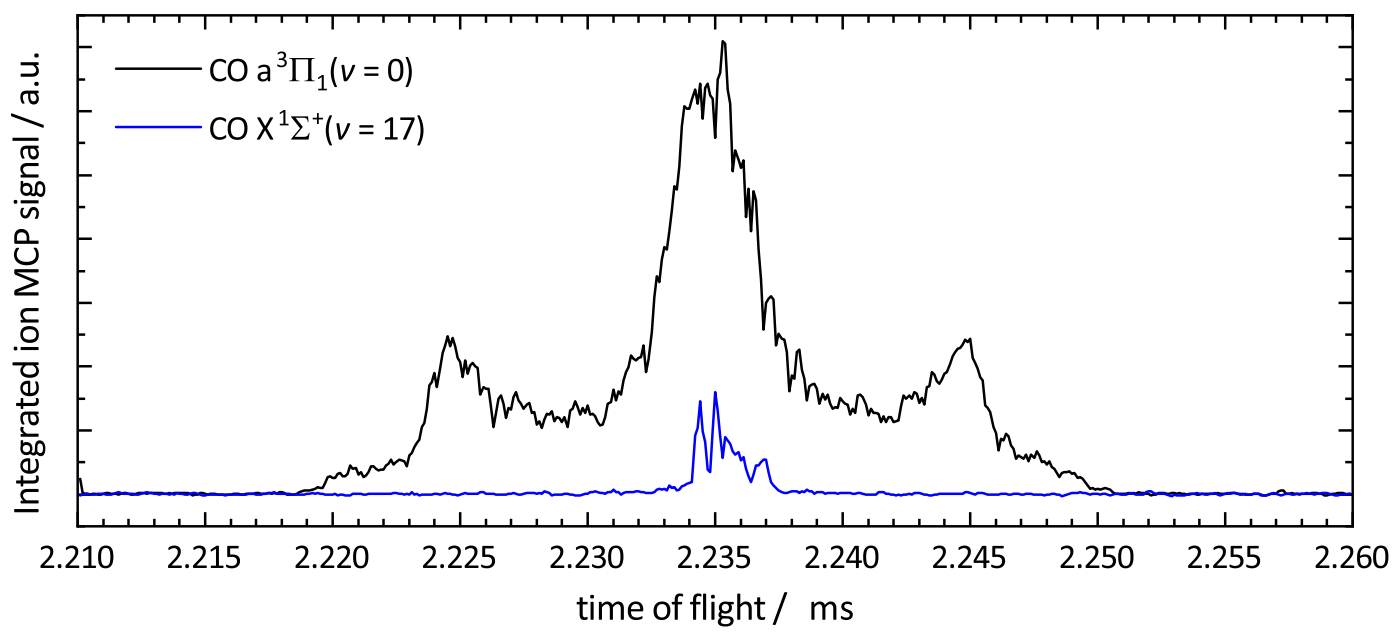

Figure 6.3: Beam profiles of Stark-manipulated molecular beams. The $x$-axis shows the flight time of the molecular beam from the point at which the first pump laser is fired (metastable production) to the point at which the beam is REMPI ionized by the probe laser. The beam velocity is $360 \mathrm{~m} / \mathrm{s}$. The decelerator is operated in guiding mode $\left(\phi_{0}=0^{\circ}\right.$, $s=3)$. The beam profile of $\mathrm{CO} \mathrm{a}^{3} \Pi_{1}$ is monitored by $(1+1)$ REMPI via the $\mathrm{k}^{3} \Pi_{1}\left(v^{\prime}=1\right) \leftarrow \mathrm{a}^{3} \Pi_{1}\left(v^{\prime \prime}=0\right)$ transition $32 \mathrm{~mm}$ downstream from the end of the decelerator, while $\mathrm{CO} \mathrm{X}^{1} \Sigma^{+}$is laser-prepared $32 \mathrm{~mm}$ behind the exit of the decelerator and monitored by $(1+1)$ REMPI via the $(1+1) \mathrm{A}^{1} \Pi\left(v^{\prime}=8\right) \leftarrow \mathrm{X}^{1} \Sigma^{+}\left(v^{\prime \prime}=17\right)$ transition $3.6 \mathrm{~mm}$ further downstream from the point of generation.

production of metastable CO prior to Stark-deceleration is challenging as it involves a spin-forbidden transition. Even though the transition gains intensity due to interaction with the $\mathrm{A}^{1} \Pi$ state, the narrow-bandwidth laser cannot saturate the transition. Third, radiative decay decreases the number of metastable CO by nearly $50 \%$ during the time that is needed to travel through the decelerator. Fourth, the preparation of ground electronic state $\mathrm{CO}$ from metastable $\mathrm{CO}$ is demanding as it involves a change of the spin manifold. This requires two more lasers and perturbed levels when employing the $\mathrm{P}^{3} \mathrm{D}$ scheme.

In order to compare Stark-manipulated beams with conventional supersonic beams, Figure 6.5 displays the REMPI spectrum of Stark-decelerated $\mathrm{CO} \mathrm{X}^{1} \Sigma^{+}(v=17)$ next to a similar spectrum obtained by using a supersonic molecular beam source without Stark decelerator. The signal-to-noise ratio decreases dramatically when the $\mathrm{CO} \mathrm{a}^{3} \Pi_{1}$ beam 


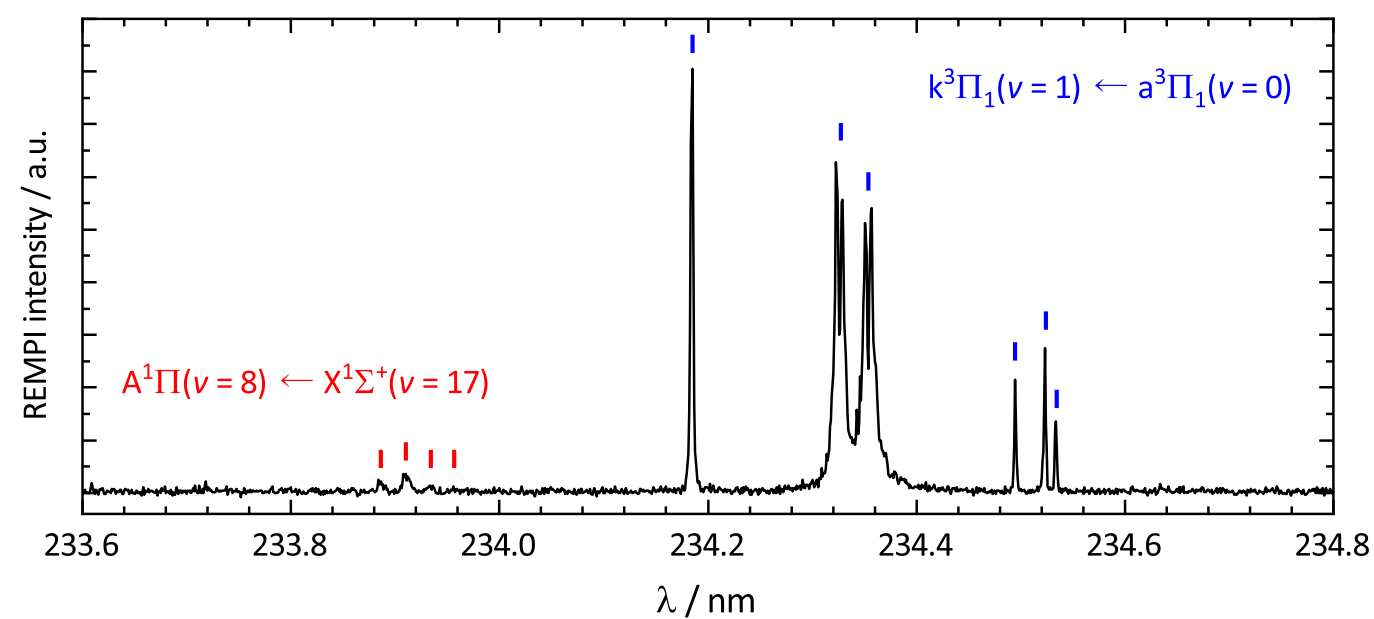

Figure 6.4: $(1+1)$ REMPI spectra of highly vibrationally excited CO (red markers, left) and metastable CO (blue markers, right). The molecular beam has been guided through the decelerator at $360 \mathrm{~m} / \mathrm{s}$ $\left(\phi_{0}=0^{\circ}, s=3\right)$. In order of ascending wavelength, the spectrum shows the $\mathrm{R}(2), \mathrm{R}(0), \mathrm{Q}(2)$, and $\mathrm{P}(2)$ (not resolved) transitions of the $(1+1) \mathrm{A}^{1} \Pi\left(v^{\prime}=8\right) \leftarrow \mathrm{X}^{1} \Sigma^{+}\left(v^{\prime \prime}=17\right)$ band, and the ${ }^{\mathrm{S}} \mathrm{R}_{32}(1)$, ${ }^{\mathrm{R}} \mathrm{R}_{22}(1),{ }^{\mathrm{Q}} \mathrm{Q}_{22}(1),{ }^{\mathrm{Q}} \mathrm{R}_{12}(1),{ }^{\mathrm{P}} \mathrm{Q}_{12}(1)$, and ${ }^{\mathrm{O}} \mathrm{P}_{12}(1)$ transitions of the $(1+1) \mathrm{k}^{3} \Pi_{1}\left(v^{\prime}=1\right) \leftarrow \mathrm{a}^{3} \Pi_{1}\left(v^{\prime \prime}=0\right)$ band. Note that the ${ }^{\mathrm{R}} \mathrm{R}_{22}(1)$ and ${ }^{\mathrm{Q}} \mathrm{Q}_{22}(1)$ transitions are split into doublets (see Section 3.4.2).

has to pass through the extra hexapole and decelerator devices before being converted to $\mathrm{CO} \mathrm{X}^{1} \Sigma^{+}(v=17)$.

For reasons of completeness, it should be mentioned that an alternative pumping scheme has been tested with the aim of producing Stark-decelerated $\mathrm{CO} \mathrm{X}^{1} \Sigma^{+}$. Following the approach developed by Blokland et al., $\mathrm{CO} \mathrm{X}^{1} \Sigma^{+}(v=0)$ can be populated by spontaneous emission from the perturbed $\mathrm{d}^{3} \Delta_{1}(v=5)$ state, which is laser-prepared by exciting the $\mathrm{d}^{3} \Delta_{1}(v=5) \leftarrow \mathrm{a}^{3} \Pi_{1}(v=0)$ transition [138]. In the Stark machine, the $\mathrm{d}^{3} \Delta_{1}(v=5) \leftarrow \mathrm{a}^{3} \Pi_{1}(v=0)$ transition is successfully monitored by laser-induced fluorescence (LIF). However, the presence of $\mathrm{CO} \mathrm{X}^{1} \Sigma^{+}(v=0)$ in the Stark-decelerated beam could not be observed by means of $(2+1)$ REMPI via the $\mathrm{B}^{1} \Sigma^{+}\left(v^{\prime}=0\right) \leftarrow \mathrm{X}^{1} \Sigma^{+}\left(v^{\prime \prime}=0\right)$ transition - the laser detection scheme of choice for quantum state-selective detection of $\mathrm{CO}$ in low $v$ states.

The successful preparation of Stark-decelerated $\mathrm{CO} \mathrm{X}^{1} \Sigma^{+}(v=17)$ is regarded as a proof of principle. However, the high- $v$ CO beam is not suited for surface scattering 

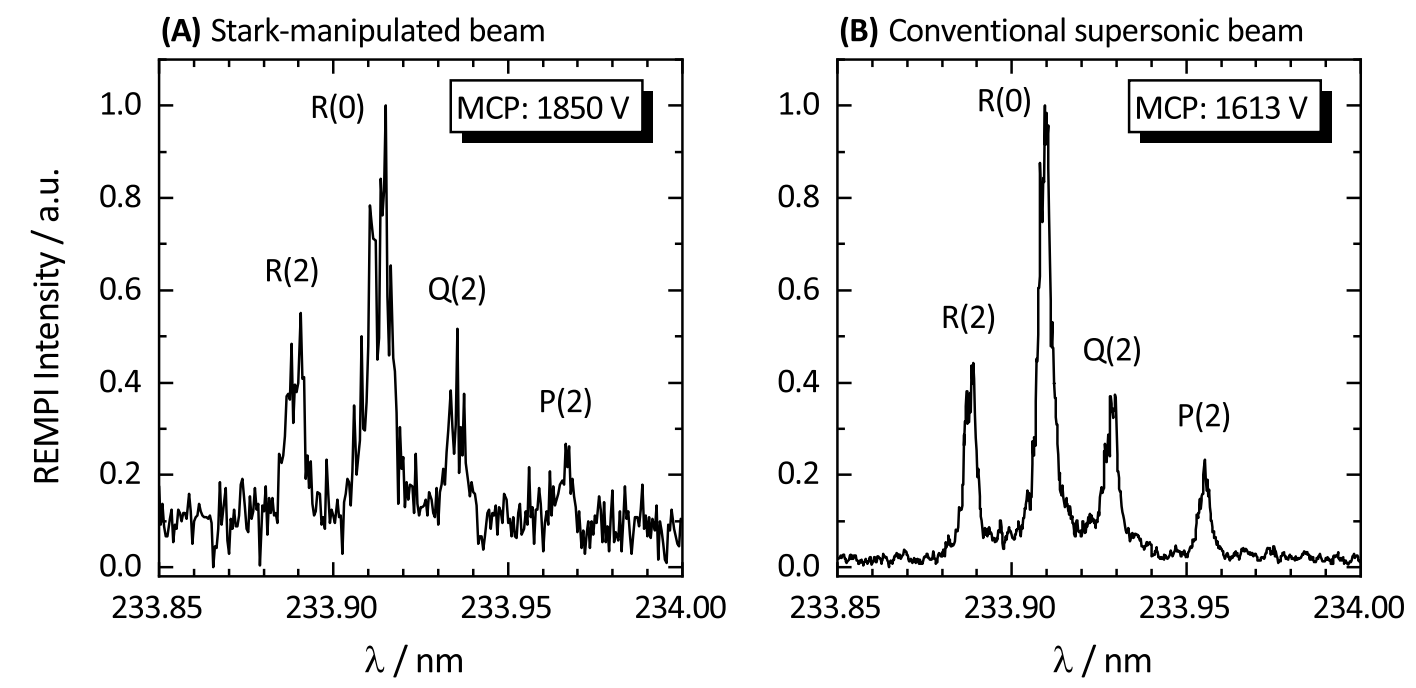

Figure 6.5: $(1+1)$ REMPI spectra via $\mathrm{A}^{1} \Pi\left(v^{\prime}=8\right) \leftarrow \mathrm{X}^{1} \Sigma^{+}\left(v^{\prime \prime}=17\right)$ of highly vibrationally excited $\mathrm{CO}$ prepared by means of $\mathrm{P}^{3} \mathrm{D}$ (dump off). Panel (A) and (B) represent typical REMPI intensities obtained for a Starkmanipulated beam and a pure supersonic beam. Laser intensities and nozzle settings are similar in both experiments. Note that the spectrum in Panel (B) is recorded at a significantly lower MCP voltage.

experiments as the total amount of $\mathrm{CO}$ prepared in $\mathrm{X}^{1} \Sigma^{+}(v=17)$ is too small. The search for surface scattered molecules using quantum state-selective REMPI was also unsuccessful, which is plausible because the population of scattered molecules spreads over several vibrational and rotational states and because the scattered beam is diluted due to temporal and angular spreading.

\subsubsection{Spatial Separation of $\mathrm{CO} \mathrm{X}^{1} \Sigma^{+}$and $\mathrm{CO} \mathrm{a}^{3} \Pi_{1}$}

In addition to REMPI spectroscopy, highly vibrationally excited molecules can also be detected by monitoring the electron emission current that results from the vibrational de-excitation at low-work function surfaces. However, since a large amount of residual $\mathrm{CO} \mathrm{a}^{3} \Pi_{1}(v=0)\left(E_{\mathrm{el}}=6.02 \mathrm{eV}\right)$ is present in the Stark-decelerated beam of $\mathrm{CO} \mathrm{X}{ }^{1} \Sigma^{+}(v=17)\left(E_{\mathrm{vib}}=4.20 \mathrm{eV}\right)$, the detection of high- $v$ CO by means of electron emission is nearly impossible because both species possess enough internal energy to overcome the surface work function $(\Phi=1.61 \mathrm{eV}$ for cesiated gold [90]). This section presents an approach to eliminate the $\mathrm{CO} \mathrm{a}^{3} \Pi_{1}$ induced background signal by spatially 


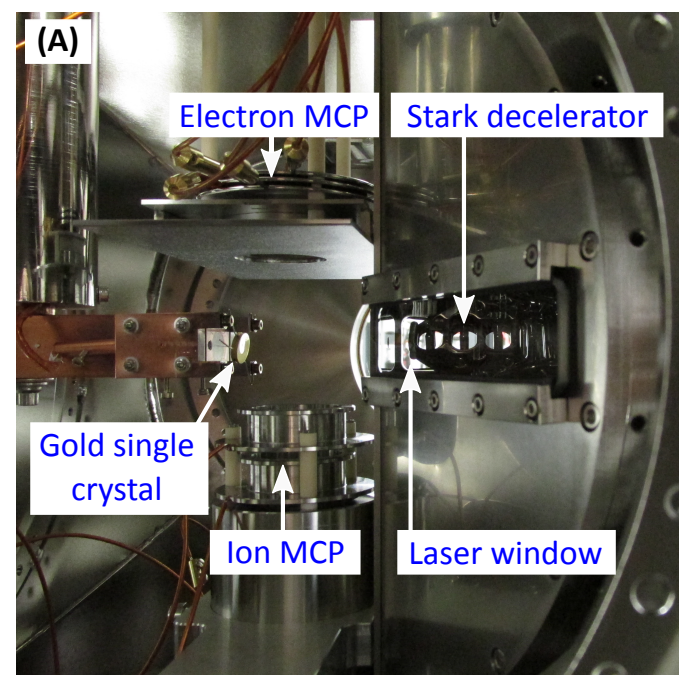

(B)

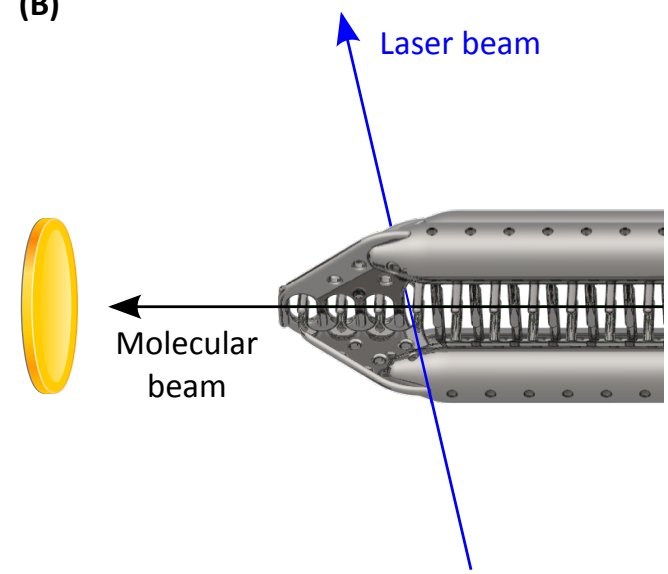

Figure 6.6: Panel (A) shows a photograph of the decelerator chamber and the surface scattering chamber. The end of the decelerator chamber is equipped with laser windows mounted at Brewster's angle. These windows allow laser access to the molecular beam before the beam has left the decelerator. Panel (B) shows a sketch illustrating laser excitation of molecules inside the decelerator.

separating high- $v$ CO from metastable CO, taking advantage of the decelerator's ability to alter the metastable beam velocity.

Spatial separation of high- $v$ CO from metastable CO can be achieved by means of time-of-flight if the velocities of the beams are different. Since $\mathrm{CO} \mathrm{a}^{3} \Pi_{1}$ exhibits a first-order Stark effect while $\mathrm{CO} \mathrm{X}^{1} \Sigma^{+}$does not, only the metastable beam velocity will change when the quickly switched electric fields of the decelerator act on the beam. In order to make this separation technique work, highly vibrationally excited CO needs to be prepared inside the Stark decelerator such that the last deceleration stages can be used to further decelerate or accelerate remaining metastables. The procedure is illustrated in Figure 6.6. Panel (A) shows a photograph of the surface scattering chamber and the decelerator chamber. Note that the decelerator chamber, which extends into the surface scattering chamber, is equipped with windows offering laser access to the last stages of the decelerator. Panel (B) schematically illustrates the laser access to the end part of the decelerator.

The separation scheme comprises three steps. First, the desired beam velocity is adjusted by using 125 of the overall 131 deceleration stages. Second, the high voltage 
is turned off when the beam is located between electrode pairs 126 and 127 (stage 126) such that the preparation lasers can be fired without causing damaging discharges. Third, the last five deceleration stages are used to alter the velocity of residual $\mathrm{CO} \mathrm{a}^{3} \Pi_{1}$ by either accelerating or decelerating the beam. The remainder of this section explains step three in more detail.

Figure 6.7 shows the arrival time of metastable $\mathrm{CO}$ at the $\mathrm{Au}(111)$ surface for different operations of the Stark decelerator. In Panel (A), the first 126 of the overall 131 electric field stages of the decelerator are used to guide the molecular beam at constant velocity. The black trace indicates the arrival time at the surface when the beam is not further manipulated while passing through the last five stages. The red (blue) trace indicates the arrival time of the beam when the last five stages are used to accelerate (decelerate) the beam. Note that the most intense part of the beam is significantly shifted to earlier (later) arrival times. Panel (B) and (C) show similar results for initially decelerated and accelerated beams, respectively.

Since CO in the ground electronic state has no linear Stark effect, the expected arrival time of $\mathrm{CO} \mathrm{X}^{1} \Sigma^{+}$generated inside the decelerator is deduced from the arrival time of metastable $\mathrm{CO}$ that has passed the last five stages with the electric fields turned off. Assuming that the $\mathrm{P}^{3} \mathrm{D}$ lasers excite the most intense part of the metastable beam, the center of the black traces shown in Figure 6.7 indicate the expected arrival time of a hypothetical $\mathrm{CO} \mathrm{X} \Sigma^{1} \Sigma^{+}$beam prepared inside the decelerator. However, it should be noted that only a small fraction of metastable $\mathrm{CO}$ can be converted to highly vibrationally excited CO. This is illustrated by molecular beam profiles depicted in Figure 6.3. Thus, a much weaker and narrower electron emission signal is expected for the quenching of $\mathrm{CO}(v=17)$ at a low-work function surface than for the quenching of $\mathrm{CO}$ $\mathrm{a}^{3} \Pi_{1}$ as depicted in Figure 6.7.

Concluding, Figure 6.7 demonstrates that the operation mode of the decelerator can be switched between guiding, deceleration, and acceleration while the molecular beam is located inside the decelerator and, thus, that the velocity of the $\mathrm{CO} \mathrm{a}^{3} \Pi_{1}$ beam can be tuned with high precision. Presumably, $\mathrm{CO} \mathrm{a}^{3} \Pi_{1}$ cannot be completely separated from $\mathrm{CO} \mathrm{X}{ }^{1} \Sigma^{+}$. However, the electron emission signal that might originate from $\mathrm{CO}$ $\mathrm{X}^{1} \Sigma^{+}(v=17)$ quenching is expected to emerge on top of a well-defined and reproducible electron emission background signal caused by $\mathrm{CO} \mathrm{a}^{3} \Pi_{1}$ when the last deceleration stages are used to alter the velocity of residual $\mathrm{CO} \mathrm{a}^{3} \Pi_{1}$. 

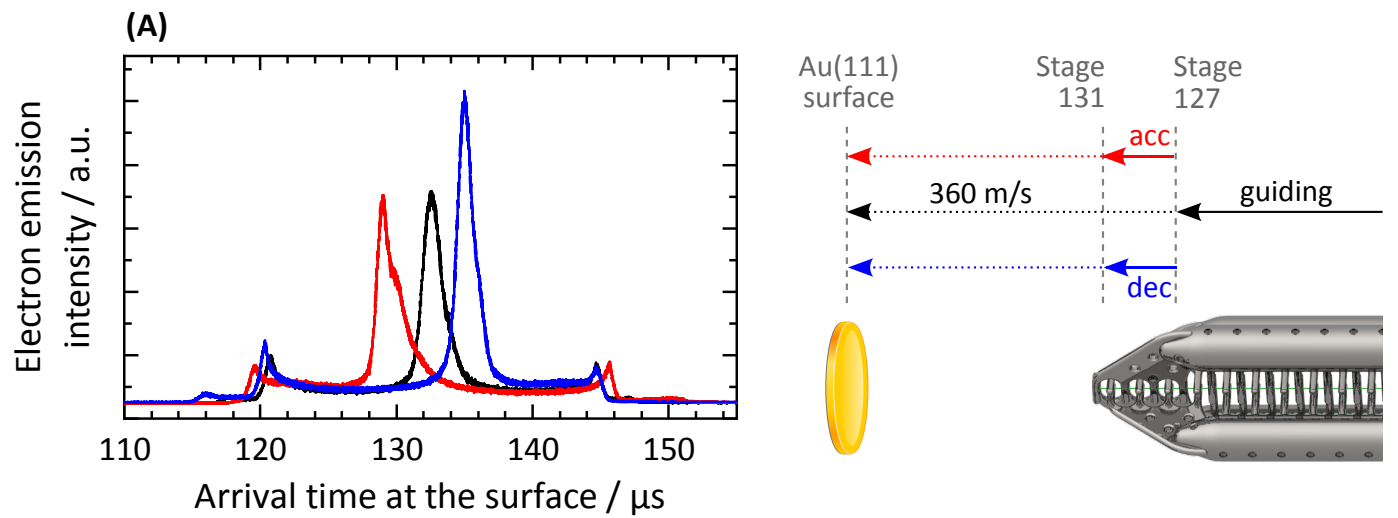

(B)
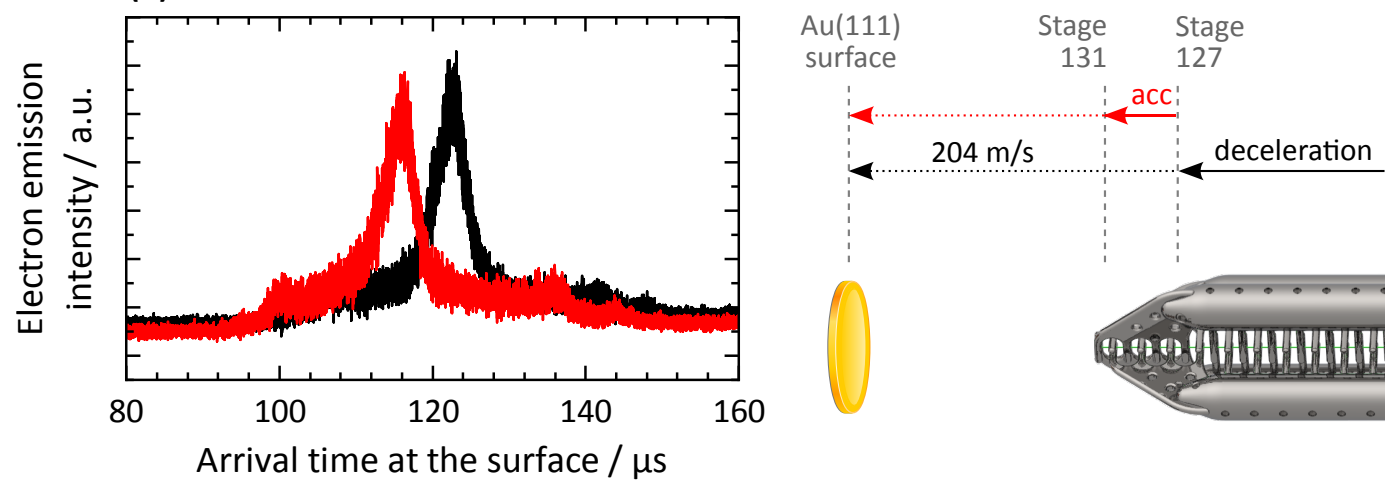

(C)
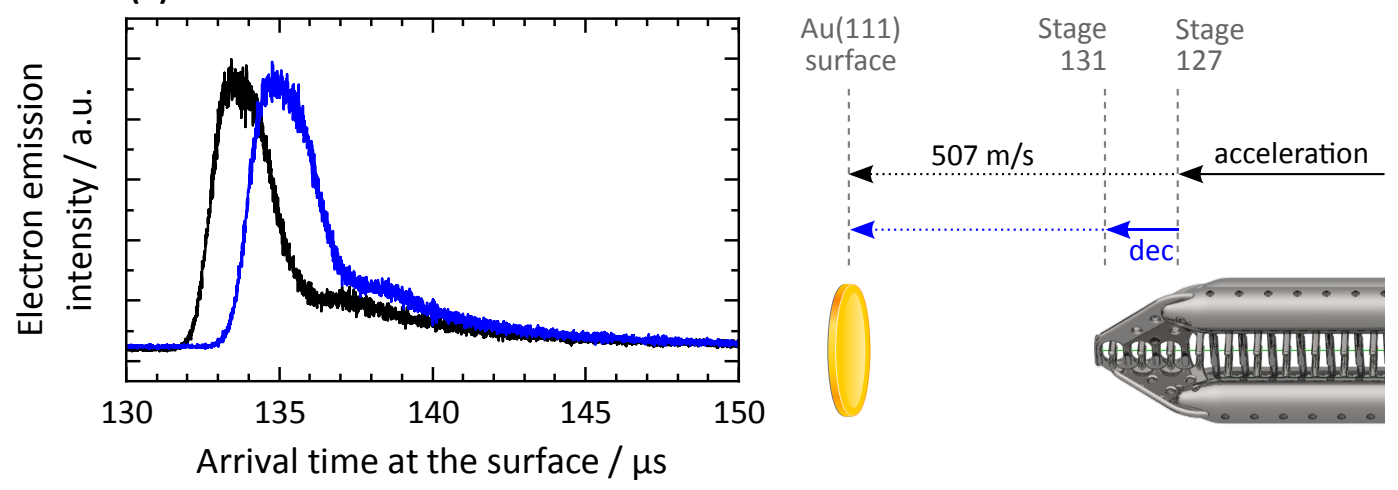

Figure 6.7: $\mathrm{CO} \mathrm{a}^{3} \Pi_{1}$ beam profiles monitored by electron emission induced by metastable quenching at $\mathrm{Au}(111)$. Traces are shown for different operations of the Stark decelerator. In Panel (A), (B), and (C), the metastable beam is guided $\left(\phi_{0}=0^{\circ}, 360 \mathrm{~m} / \mathrm{s}\right)$, decelerated $\left(\phi_{0}=47^{\circ}, 204 \mathrm{~m} / \mathrm{s}\right)$, and accelerated $\left(\phi_{0}=-90^{\circ}, 507 \mathrm{~m} / \mathrm{s}\right)$, respectively, using 126 out of the overall 131 stages of the decelerator (black). The last five stages can be used to either accelerate $\left(\phi_{0}=-90^{\circ}\right.$, "acc", red) or decelerate $\left(\phi_{0}=78^{\circ}\right.$, "dec", blue) the beam. Solid arrows indicate Stark manipulation of the beam with the decelerator's electrodes set under high voltage, while dotted arrows indicate propagation of the beam with the electric fields turned off. 



\section{Conclusion}

The scattering of highly vibrationally excited $\mathrm{CO} \mathrm{X}^{1} \Sigma^{+}(v=17, J=0)$ molecules from clean gold and silver surfaces is investigated for the first time [31, 32]. Supersonic molecular beams of CO are scattered from the (111) faces of gold and silver single crystals which are kept under ultra-high vacuum conditions. Quantum state-selective preparation and detection of $\mathrm{CO}$ is accomplished by laser spectroscopic methods. A three-laser optical excitation scheme is used to prepare incident $\mathrm{CO}$ in a high $v$ state prior to surface scattering [106]. It has been demonstrated previously that the surface dynamics of highly vibrationally excited diatomic molecules may be strongly dominated by electronically non-adiabatic effects [25]. This makes highly vibrationally excited molecules valuable candidates for surface scattering studies that are aiming at a deeper and fundamental understanding of non-adiabatic surface dynamics.

The molecule-surface experiments described in the present thesis include the measurement of state-to-state time-of-flight distributions, state-to-state angular distributions, final rotational state distributions, and final vibrational state distributions. The translational energy of incident molecules is varied between $0.27 \mathrm{eV}$ and $0.57 \mathrm{eV}$.

The final translational energy of vibrationally and rotationally elastically scattered molecules obeys the Baule limit, which agrees with a direct scattering mechanism. Narrow angular distributions as well as hyperthermal rotational distributions of both vibrationally eleastically and inelastically scattered molecules are also consistent with a direct scattering channel.

Rotational state distributions do not show any evidence of rotational rainbows similar to those observed in the scattering of highly vibrationally excited NO at $\mathrm{Au}(111)$ [87].

The main part of this surface-scattering study focuses on the dissipation of the molecule's vibrational energy. The employed resonance-enhanced multi-photon ionization detection scheme allows the detection of final vibrational states in the range $14 \geq v \geq 18$. For the scattering from $\mathrm{Au}(111)$, scattering products collectively populate vibrational states in the range $14 \geq v \geq 17$. For the scattering from $\operatorname{Ag}(111)$, however, CO relaxes to vibrational levels below $v=14$, which cannot be detected directly. Yet, the total number of scattered molecules, which is needed to determine the vibrational relaxation probability at $\mathrm{Ag}(111)$, is derived from scattering experiments employing a 


\section{Conclusion}

chlorinated $\operatorname{Ag}(111)$ surface, on which vibrational relaxation is suppressed. In general, vibrational relaxation probabilities are found to be higher for silver than for gold. Vibrational relaxation probabilities tend to increase with incidence translational energy for both metals. The observed incidence translational energy dependence is more pronounced for gold than for silver. Vibrational excitation to $v=18$ or higher states is not observed.

In previous work, the dynamics of highly vibrationally excited diatomic molecules in collisions with metal surfaces has been studied only for the NO molecule. Together with results reported for $\mathrm{NO}$ and $\mathrm{CO}$ prepared in low $v$ states, a large amount of data is available now, describing vibrational relaxation processes in direct molecule-surface scattering events. The data presented in the present work completes a set of data that spans two molecules ( $\mathrm{CO}$ and $\mathrm{NO}$ ), two metal surfaces ( $\mathrm{Au}$ and $\mathrm{Ag}$ ), and different incident vibrational states ranging from $v=2$ to $v=17$.

Key results in the vibrational relaxation of $\mathrm{CO}$ and $\mathrm{NO}$ at $\mathrm{Au}(111)$ and $\mathrm{Ag}(111)$ are as follows. First, vibrational relaxation probabilities are higher for $\operatorname{Ag}(111)$ than for $\mathrm{Au}(111)$ due to the lower work function, $\Phi$, of silver. Second, the vibrational relaxation probability on $\mathrm{NO}$ is higher than that on $\mathrm{CO}$ for similar incident vibrational states due to the higher electron affinity of NO. Third, the vibrational relaxation probability increases with incident vibrational excitation due to an increase of the vertical electron binding energy at the outer classical turning point, $E\left(r_{\text {out }}\left(v_{\text {inc }}\right)\right)$, with vibrational excitation.

The third point demonstrates that the vibrational state dependent vertical electron binding energy needs to be considered rather than just the electron affinity. The vertical electron binding energy and the electron affinity are closely related because $E\left(r_{\text {out }}\left(v_{\text {inc }}\right)\right)$ depends on the vertical shift of the anionic gas-phase potential energy curve of the diatomic molecule with respect to the neutral. Roughly speaking, the higher the electron affinity, the higher $E\left(r_{\text {out }}\left(v_{\text {inc }}\right)\right)$. Apart from the vertical shift, $E\left(r_{\text {out }}\left(v_{\text {inc }}\right)\right)$ also depends on the horizontal shift of the potential energy curves. The vertical electron binding energy at the outer turning point generally increases when the anion's equilibrium bond length is shifted to larger internuclear distances than that of the neutral species. This is the case for NO, in which the anion has a larger bond length than the neutral species, but not for $\mathrm{CO}$, in which the anionic and neutral species have similar bond lengths. Furthermore, $E\left(r_{\text {out }}\left(v_{\text {inc }}\right)\right)$ varies with shape and slope of the neutral and anionic potential energy curves.

According to the results mentioned above, the vibrational relaxation dynamics of molecule-surface systems considered in the present work is characterized by the vertical electron binding energy of the molecule and the work function of the surface. By comparing different molecule-surface systems, a unifying relationship has been found 
between the vibrational relaxation probability and $E\left(r_{\text {out }}\left(v_{\text {inc }}\right)\right)-\Phi$. Plotting the vibrational relaxation probability as a function of $E\left(r_{\text {out }}\left(v_{\text {inc }}\right)\right)-\Phi$ yields a sigmoidal curve (Figure 5.24). For an incidence translational energy of $0.6 \mathrm{eV}$, the vibrational relaxation probability increases from 0 to 1 in the window $-6.0 \mathrm{eV} \lesssim E\left(r_{\text {out }}\left(v_{\text {inc }}\right)\right)-\Phi \lesssim$ $-3.0 \mathrm{eV}$. Molecule-surface systems characterized by $E\left(r_{\text {out }}\left(v_{\text {inc }}\right)\right)-\Phi \lesssim-6.0 \mathrm{eV}$ show very little vibrational relaxation, while molecule-surface systems characterized by $E\left(r_{\text {out }}\left(v_{\text {inc }}\right)\right)-\Phi \gtrsim-3.0 \mathrm{eV}$ show complete vibrational relaxation.

$\Phi$ and $E\left(r_{\text {out }}\left(v_{\text {inc }}\right)\right)$ are energetic quantities relevant to an electron transfer from the surface to the molecule. Electron transfer is likely to occur as soon as the electron binding energy overcomes the work function. Thus, the difference $E\left(r_{\text {out }}\left(v_{\text {inc }}\right)\right)-\Phi$ represents the amount of image charge stabilization by which the affinity level of the molecule needs to be lowered in energy to enable the electron transfer. In other words, $E\left(r_{\text {out }}\left(v_{\text {inc }}\right)\right)-\Phi$ is a measure of how close a molecule needs to approach the surface before it reaches the region where the neutral and anionic diabats cross. As soon as electronically non-adiabatic transitions occur near this crossing seam, the molecule may suffer vibrational relaxation.

The dependence of the vibrational relaxation probability on $\Phi$ and $E\left(r_{\text {out }}\left(v_{\text {inc }}\right)\right)$ strongly suggests that the vibrational relaxation of diatomic molecules at metal surfaces proceeds electronically non-adiabatically and that an electron transfer process from the surface to the molecule, forming a transient anionic species, lies at the heart of the vibrational relaxation mechanism.

Summarizing, two consequences of the empirically found relationship between the vibrational relaxation probability and $E\left(r_{\text {out }}\left(v_{\text {inc }}\right)\right)-\Phi$ shall be emphasized. First, the vibrational relaxation probability can be predicted for a certain molecule-surface system simply by evaluating easily accessible energetic properties of the isolated molecule and surface. Second, molecule-surface systems for which electron transfer processes must be considered explicitly when describing their electronically non-adiabatic surface dynamics can easily be identified based on the work function of the surface and the vertical electron binding energy of the molecule. This might be of special importance for theorists when modeling non-adiabatic dynamics of certain molecule-surface systems. Predicting accurate final vibrational state distributions and correct trends in the velocity dependence of the relaxation probability is still a challenge to theory [30]. Molecule-surface systems with low work functions and high electron binding energies may be best described by theoretical approaches based on the strong vibration-electron coupling limit, such as the independent-electron surface hopping approach. Electronic friction based models, however, may apply best to molecule-surface systems which exhibit weak non-adiabatic couplings. See for example recent work by Spiering and Meyer, 


\section{Conclusion}

who calculate vibrational de-excitation probabilities for $\mathrm{H}_{2}$ in collisions with $\mathrm{Cu}(111)$ $\left(E\left(r_{\text {out }}\left(v_{\text {inc }}\right)\right)-\Phi \approx-7.0 \mathrm{eV}\right)[97]$. Miao, Dou and Subotnik recently introduced an approach that interpolates between the weak and the strong coupling cases and demonstrated that electronic friction alone is not sufficient to accurately predict the dynamics of highly vibrationally excited NO at gold [94]. Thus, the novel results presented for the surface-scattering of highly vibrationally excited $\mathrm{CO}$ together with the comparison to a large number of other results that have accumulated for various molecule-surface systems in the group of Prof. Wodtke over several years hopefully help to improve existing theoretical models and contribute to a fundamental understanding of electronically non-adiabatic surface dynamics.

It is in that spirit that experiments were planned to investigate the dynamics of highly vibrationally excited $\mathrm{CO}$ at low-work function surfaces, where electron emission is expected during the vibrational de-excitation process because the vibrational energy exceeds the work function [88]. Since similar experiments for NO revealed an inverse velocity dependence of the electron emission yield, a newly developed Stark deceleratorbased beam-surface scattering setup was intended for these experiments. The setup was modified in two ways. First, a method was developed that allows surface scattering experiments in which the velocity of the molecular beam can be scanned between $100 \mathrm{~m} / \mathrm{s}$ and $500 \mathrm{~m} / \mathrm{s}$ by repeating the scattering experiment with any desired scanning interval. Second, decelerator settings were adjusted such that highly vibrationally excited $\mathrm{CO}$ potentially produced inside the decelerator device can be separated from residual metastable CO. Unfortunately, the number of molecules leaving the Stark decelerator is too small to apply the $\mathrm{P}^{3} \mathrm{D}$ pumping scheme for $\mathrm{CO}(v=17)$ production [106] and to subsequently subject those highly vibrationally excited molecules to a surface scattering experiment. 


\section{A Appendix}

\section{A.1 Rotational Transition Wavenumbers of the $A^{1} \Pi-X^{1} \Sigma^{+}$ System in CO}

Table A.1: Rotational transitions of the 7-17 $\left(v^{\prime}-v^{\prime \prime}\right)$ band and the 7-16 band in $\mathrm{cm}^{-1}$ experimentally determined via $(1+1) \mathrm{A}^{1} \Pi\left(v^{\prime}, J^{\prime}\right) \leftarrow \mathrm{X}^{1} \Sigma^{+}\left(v^{\prime \prime}, J^{\prime \prime}\right)$ REMPI spectroscopy.

\begin{tabular}{|c|c|c|c|}
\hline \multicolumn{4}{|c|}{$7-17$} \\
\hline$J^{\prime \prime}$ & $\mathrm{P}\left(J^{\prime \prime}\right)$ & $\mathrm{Q}\left(J^{\prime \prime}\right)$ & $\mathrm{R}\left(J^{\prime \prime}\right)$ \\
\hline 0 & & & 41511.58 \\
\hline 1 & & 41508.33 & 41514.09 \\
\hline 2 & 41501.83 & 41507.59 & 41516.22 \\
\hline 3 & 41497.83 & 41506.47 & 41517.99 \\
\hline 4 & 41493.47 & 41504.99 & 41519.38 \\
\hline 5 & 41488.74 & 41503.13 & 41520.40 \\
\hline 6 & 41483.63 & 41500.90 & 41521.04 \\
\hline 7 & 41478.15 & 41498.30 & 41521.31 \\
\hline 8 & 41472.30 & 41495.32 & 41521.21 \\
\hline 9 & 41466.08 & 41491.97 & 41520.73 \\
\hline 10 & 41459.49 & 41488.25 & 41519.88 \\
\hline 11 & 41452.53 & 41484.16 & 41518.65 \\
\hline 12 & 41445.19 & 41479.69 & 41517.05 \\
\hline 13 & 41437.48 & 41474.84 & 41515.06 \\
\hline 14 & 41429.40 & 41469.62 & 41512.70 \\
\hline 15 & 41420.95 & 41464.03 & 41509.96 \\
\hline 16 & 41412.12 & 41458.05 & 41506.84 \\
\hline 17 & 41402.92 & 41451.70 & 41503.34 \\
\hline 18 & 41393.34 & 41444.97 & 41499.46 \\
\hline 19 & 41383.38 & 41437.87 & 41495.19 \\
\hline
\end{tabular}


Table A.1: (continued)

\begin{tabular}{|c|c|c|c|}
\hline$J^{\prime \prime}$ & $\mathrm{P}\left(J^{\prime \prime}\right)$ & $\mathrm{Q}\left(J^{\prime \prime}\right)$ & $\mathrm{R}\left(J^{\prime \prime}\right)$ \\
\hline 20 & 41373.05 & 41430.38 & 41490.54 \\
\hline 21 & 41362.34 & 41422.51 & 41485.51 \\
\hline 22 & 41351.26 & 41414.26 & 41480.08 \\
\hline 23 & 41339.79 & 41405.62 & 41474.27 \\
\hline 24 & 41327.95 & 41396.60 & 41468.08 \\
\hline 25 & 41315.72 & 41387.20 & $41464.56,41460.81$ \\
\hline 26 & 41303.12 & 41377.41 & 41454.51 \\
\hline 27 & $41292.81,41289.24$ & 41367.23 & 41447.14 \\
\hline 28 & 41277.21 & 41356.66 & 41439.37 \\
\hline 29 & 41263.26 & $41350.54,41343.33$ & 41431.21 \\
\hline 30 & 41249.05 & 41334.36 & 41422.65 \\
\hline 31 & 41234.51 & 41322.61 & 41413.69 \\
\hline 32 & 41219.49 & 41310.48 & 41404.33 \\
\hline 33 & 41203.62 & 41297.52 & 41394.57 \\
\hline 34 & 41188.71 & 41285.59 & 41384.40 \\
\hline 35 & 41172.29 & 41271.90 & 41373.83 \\
\hline 36 & 41155.11 & 41258.55 & 41362.85 \\
\hline 37 & 41140.26 & 41243.89 & 41351.46 \\
\hline 38 & 41122.00 & $41234.40,41227.85$ & 41339.67 \\
\hline 39 & 41103.67 & $41217.71,41209.58$ & 41327.45 \\
\hline 40 & 41088.93 & 41199.77 & 41314.82 \\
\hline 41 & & 41183.42 & $41309.69,41301.00$ \\
\hline 42 & & $41174.69,41165.20$ & 41289.67 \\
\hline 43 & & $41151.64,41146.66$ & 41275.32 \\
\hline 44 & & 41134.20 & 41260.83 \\
\hline 45 & & 41116.54 & 41246.12 \\
\hline 46 & & 41098.06 & 41231.58 \\
\hline \multicolumn{4}{|c|}{$7-16$} \\
\hline$J^{\prime \prime}$ & $\mathrm{P}\left(J^{\prime \prime}\right)$ & $\mathrm{Q}\left(J^{\prime \prime}\right)$ & $\mathrm{R}\left(J^{\prime \prime}\right)$ \\
\hline 0 & & & 43239.98 \\
\hline 1 & & 43236.69 & 43242.45 \\
\hline 2 & 43230.12 & 43235.88 & 43244.52 \\
\hline 3 & 43226.03 & 43234.66 & 43246.18 \\
\hline
\end{tabular}


A.1 Rotational Transition Wavenumbers of the $A^{1} \Pi-X^{1} \Sigma^{+}$System in $C O$

Table A.1: (continued)

\begin{tabular}{|c|c|c|c|}
\hline$J^{\prime \prime}$ & $\mathrm{P}\left(J^{\prime \prime}\right)$ & $\mathrm{Q}\left(J^{\prime \prime}\right)$ & $\mathrm{R}\left(J^{\prime \prime}\right)$ \\
\hline 4 & 43221.52 & 43233.04 & 43247.43 \\
\hline 5 & 43216.61 & 43231.00 & 43248.27 \\
\hline 6 & 43211.30 & 43228.56 & 43248.71 \\
\hline 7 & 43205.57 & 43225.72 & 43248.74 \\
\hline 8 & 43199.45 & 43222.46 & 43248.35 \\
\hline 9 & 43192.91 & 43218.80 & 43247.56 \\
\hline 10 & 43185.97 & 43214.73 & 43246.36 \\
\hline 11 & 43178.62 & 43210.25 & 43244.75 \\
\hline 12 & 43170.87 & 43205.36 & 43242.72 \\
\hline 13 & 43162.71 & 43200.07 & 43240.29 \\
\hline 14 & 43154.14 & 43194.36 & 43237.44 \\
\hline 15 & 43145.16 & 43188.24 & 43234.17 \\
\hline 16 & 43135.77 & 43181.70 & 43230.49 \\
\hline 17 & 43125.97 & 43174.76 & 43226.40 \\
\hline 18 & 43115.76 & 43167.40 & 43221.88 \\
\hline 19 & 43105.15 & 43159.63 & 43216.95 \\
\hline 20 & 43094.52 & 43151.44 & 43211.61 \\
\hline 21 & 43083.04 & 43142.84 & 43205.84 \\
\hline 22 & 43071.52 & 43133.82 & 43199.65 \\
\hline 23 & 43058.96 & 43124.38 & 43193.03 \\
\hline 24 & 43046.26 & 43114.52 & 43186.00 \\
\hline 25 & 43033.03 & 43104.25 & $43181.61,43177.86$ \\
\hline 26 & 43019.49 & 43093.83 & 43170.65 \\
\hline 27 & $43008.00,43004.43$ & 43082.5 & 43162.33 \\
\hline 28 & 42991.43 & 43070.79 & 43153.59 \\
\hline 29 & 42976.46 & $43063.74,43056.53$ & 43144.41 \\
\hline 30 & 42961.20 & 43047.13 & 43134.80 \\
\hline 31 & 42945.58 & 43034.18 & 43124.76 \\
\hline 32 & 42929.44 & 43020.71 & 43114.28 \\
\hline 33 & 42912.42 & 43006.32 & 43103.37 \\
\hline 34 & 42896.32 & 42993.20 & 43091.54 \\
\hline 35 & 42878.68 & 42978.29 & 43081.79 \\
\hline 36 & 42860.24 & 42963.68 & 43068.62 \\
\hline
\end{tabular}


A Appendix

Table A.1: (continued)

\begin{tabular}{|c|c|c|c|}
\hline$J^{\prime \prime}$ & $\mathrm{P}\left(J^{\prime \prime}\right)$ & $\mathrm{Q}\left(J^{\prime \prime}\right)$ & $\mathrm{R}\left(J^{\prime \prime}\right)$ \\
\hline 37 & 42844.10 & 42947.73 & 43055.42 \\
\hline 38 & 42824.51 & $42936.91,42930.36$ & 43045.91 \\
\hline 39 & 42804.81 & $42918.85,42910.72$ & 43029.46 \\
\hline 40 & 42788.68 & 42899.52 & 43015.12 \\
\hline 41 & & 42881.73 & $43008.00,42999.31$ \\
\hline 42 & & $42871.53,42862.04$ & 42986.51 \\
\hline 43 & & $42846.98,42842.00$ & 42970.66 \\
\hline 44 & & 42828.00 & 42954.63 \\
\hline 45 & & 42808.77 & 42938.35 \\
\hline 46 & & 42788.68 & 42922.20 \\
\hline
\end{tabular}




\section{List of Abbreviations}

$\mathrm{AC}$

a.u.

AES

AFM

amu

$\mathrm{BBO}$

$\mathrm{BO}$

$\mathrm{BOA}$

$\mathrm{CW}$

DFT

EF

EHP

ET

et al.

etc.

$\mathrm{eV}$

Fig.

GGA

hcp

$\mathrm{HV}$

ICS

i.e.

IESH

KDP

KTP

LEED

LIF

LZ

$\mathrm{MCP}$

Nd:YAG alternating current

arbitrary units

Auger electron spectroscopy

atomic force microscopy

atomic mass unit

$\beta$-barium borate

Born-Oppenheimer

Born-Oppenheimer approximation

continuous-wave

density functional theory

electronic friction

electron-hole pair

electron transfer

et alii or et aliae

et cetera

electron volt (unit)

Figure

generalized gradient approximation

hexagonal close-packed

high voltage

image charge stabilization

id est

independent-electron surface hopping

potassium dideuterium phosphate

potassium titanyl phosphate

low-energy electron diffraction

laser induced fluorescence

Landau-Zener

multi channel plate

neodymium-doped yttrium aluminum garnet 
List of Abbreviations

OPO optical parametric oscillator

p. page

$\mathrm{PC} \quad$ personal computer

$\mathrm{PD} \quad$ photo diode

PDL pulsed dye laser

PES potential energy surface

PW91 Perdew-Wang 91 functional

RAIRS reflection absorption infrared spectroscopy

RAT Rydberg atom tagging

Ref. Reference

REMPI resonance-enhanced multi-photon ionization

RGA residual gas analyzer

SERS surface enhanced Raman spectroscopy

SFG sum frequency generation

STM scanning tunneling microscopy

TD trapping-desorption

TPD temperature programmed desorption

UHV ultra-high vacuum

UPS

ultraviolet photoelectron spectroscopy

UV ultraviolet

VMI velocity-map imaging

VUV vacuum ultraviolet

XPS X-ray photoelectron spectroscopy 


\section{Bibliography}

[1] G. Ertl, Reactions at Surfaces: From Atoms to Complexity (Nobel Lecture), Angew. Chem. Int. Ed. 2008, 47, 3524-3535, https://doi.org/10.1002/anie. 200800480 .

[2] J. W. Döbereiner, Briefwechsel zwischen Goethe und Johann Wolfgang Döbereiner (1810-1830), edited by J. Schiff, Hermann Böhlaus Nachfolger, Weimar, Letter 94, July 1823, p. 78, 1914, https://archive.thulb.uni-jena.de/collections/receive/ HisBest_cbu_00025461.

[3] G. B. Kauffman, Johann Wolfgang Döbereiner's Feuerzeug, On the Sesquicentennial Anniversary of his Death, Platinum Metals Rev. 1999, 43, 122-128.

[4] W. Ostwald, Über Katalyse, in Vorträge und Diskussionen von der 73. Naturforscherversammlung zu Hamburg, Phys. Z. 1902, 3, 313-322.

[5] J. H. Sinfelt, Role of Surface Science in Catalysis, Surf. Sci. 2002, 500, 923-946, https://doi.org/10.1016/S0039-6028(01)01532-1.

[6] I. Langmuir, Part II.- "Heterogeneous Reactions." Chemical Reactions on Surfaces, Trans. Faraday Soc. 1922, 17, 607-620, http://dx.doi.org/10.1039/ TF9221700607.

[7] G. Ertl, Molecules at Solid Surfaces: A Personal Reminiscence, Ann. Rev. Phys. Chem. 2017, 68, 1-17, https://doi.org/10.1146/ annurev-physchem-052516-044758.

[8] G. A. Somorjai, Y. Li, Impact of Surface Chemistry, Proc. Natl. Acad. Sci. 2011, 108, 917-924, https://doi.org/10.1073/pnas.1006669107.

[9] X. Yang, A. M. Wodtke, Surface Reaction Dynamics, Chem. Soc. Rev. 2016, 45, 3573-3575, http://dx.doi.org/10.1039/C6CS90049F.

[10] H. Eyring, M. Polanyi, Über einfache Gasreaktionen, Z. physikal. Chem. B 1931, 12(4), 279-311. 
Bibliography

[11] H. Eyring, H. Gershinowitz, C. E. Sun, The Absolute Rate of Homogeneous Atomic Reactions, J. Chem. Phys. 1935, 3, 786-796, https://doi.org/10.1063/ 1.1749593.

[12] M. Born, R. Oppenheimer, Zur Quantentheorie der Molekeln, Annalen der Physik 1927, 389(20), 457-484, https://doi.org/10.1002/andp.19273892002.

[13] X. Yang, D. H. Zhang, Probing Quantum Dynamics of Elementary Chemical Reactions via Accurate Potential Energy Surfaces, Z. Phys. Chem. 2013, 227, 1247-1265, https://doi.org/10.1524/zpch.2013.0424.

[14] D. W. Chandler, P. L. Houston, Two-Dimensional Imaging of State-Selected Photodissociation Products Detected by Multiphoton Ionization, J. Chem. Phys. 1987, 87(2), 1445-1447, https://doi.org/10.1063/1.453276.

[15] M. N. R. Ashfold, N. H. Nahler, A. J. Orr-Ewing, O. P. J. Vieuxmaire, R. L. Toomes, T. N. Kitsopoulos, I. A. Garcia, D. A. Chestakov, S.-M. Wu, D. H. Parker, Imaging the Dynamics of Gas Phase Reactions, Phys. Chem. Chem. Phys. 2006, 8, 26-53, http://dx.doi.org/10.1039/B509304J.

[16] L. Schnieder, W. Meier, K. H. Welge, M. N. R. Ashfold, C. M. Western, Photodissociation Dynamics of $\mathrm{H}_{2} \mathrm{~S}$ at $121.6 \mathrm{~nm}$ and a Determination of the Potential Energy Function of $\mathrm{SH}\left(\mathrm{A}^{2} \Sigma^{+}\right)$, J. Chem. Phys. 1990, 92, 7027-7037, https://doi.org/10.1063/1.458243.

[17] H. L. Bethlem, G. Berden, G. Meijer, Decelerating Neutral Dipolar Molecules, Phys. Rev. Lett. 1999, 83, 1558-1561, https://doi.org/10.1103/PhysRevLett.83. 1558.

[18] S. Y. T. van de Meerakker, H. L. Bethlem, N. Vanhaecke, G. Meijer, Manipulation and Control of Molecular Beams, Chem. Rev. 2012, 112, 4828-4878, https://doi. org/10.1021/cr200349r.

[19] A. von Zastrow, J. Onvlee, S. N. Vogels, G. C. Groenenboom, A. van der Avoird, S. Y. T. van de Meerakker, State-Resolved Diffraction Oscillations Imaged for Inelastic Collisions of NO Radicals With He, Ne and Ar, Nature Chem. 2014, 6, 216-221, https://doi.org/10.1038/nchem.1860.

[20] E. I. Dashevskaya, I. Litvin, E. E. Nikitin, J. Troe, Electronically Nonadiabatic Mechanism of the Vibrational Relaxation of NO in Ar: Rate Coefficients From $A b$ Initio Potentials and Asymptotic Coupling, J. Chem. Phys. 2018, 149, 014301, https://doi.org/10.1063/1.5038619. 
[21] A. M. Wodtke, J. C. Tully, D. J. Auerbach, Electronically Non-Adiabatic Interactions of Molecules at Metal Surfaces: Can We Trust the Born-Oppenheimer Approximation for Surface Chemistry?, Int. Rev. Phys. Chem. 2004, 23, 513-539, https://doi.org/10.1080/01442350500037521.

[22] E. Hasselbrink, How Non-Adiabatic are Surface Dynamical Processes?, Curr. Opin. Solid State Mater. Sci. 2006, 10, 192-204, https://doi.org/10.1016/j. cossms.2007.04.003.

[23] B. I. Lundqvist, A. Hellmann, I. Zorić, Electron Transfer and Nonadiabaticity, in Handbook of Surface Science, Dynamics, Volume 3, Chapter 10, series editors: S. Holloway and N. V. Richardson, volume editors: E. Hasselbrink and B. I. Lundqvist, Elsevier, North-Holland, pp. 429-524, 2008, https://doi.org/10.1016/ S1573-4331(08)00010-3.

[24] O. Bünermann, H. Jiang, Y. Dorenkamp, A. Kandratsenka, S. M. Janke, D. J. Auerbach, A. M. Wodtke, Electron-Hole Pair Excitation Determines the Mechanism of Hydrogen Atom Adsorption, Science 2015, 350, 1346-1349, https: //doi.org/10.1126/science.aad4972.

[25] Y. Huang, C. T. Rettner, D. J. Auerbach, A. M. Wodtke, Vibrational Promotion of Electron Transfer, Science 2000, 290, 111-114, https://doi.org/10.1126/science. 290.5489 .111$.

[26] M. Head-Gordon, J. C. Tully, Molecular Dynamics with Electronic Frictions, J. Chem. Phys. 1995, 103, 10137-10145, https://doi.org/10.1063/1.469915.

[27] S. Monturet, P. Saalfrank, Role of Electronic Friction During the Scattering of Vibrationally Excited Nitric Oxide Molecules from Au(111), Phys. Rev. B 2010, 82, 075404, https://doi.org/10.1103/PhysRevB.82.075404.

[28] N. Shenvi, S. Roy, J. C. Tully, Nonadiabatic Dynamics at Metal Surfaces: Independent-Electron Surface Hopping, J. Chem. Phys. 2009, 130, 174107, https://doi.org/10.1063/1.3125436.

[29] N. Shenvi, S. Roy, J. C. Tully, Dynamical Steering and Electronic Excitation in NO Scattering from a Gold Surface, Science 2009, 326, 829-832, https://doi.org/ 10.1126/science.1179240.

[30] B. C. Krüger, N. Bartels, C. Bartels, A. Kandratsenka, J. C. Tully, A. M. Wodtke, T. Schäfer, NO Vibrational Energy Transfer on a Metal Surface: Still a Challenge 
Bibliography

to First-Principles Theory, J. Phys. Chem. C 2015, 119, 3268-3272, https://doi. org/10.1021/acs.jpcc.5b00388.

[31] R. J. V. Wagner, N. Henning, B. C. Krüger, G. B. Park, J. Altschäffel, A. Kandratsenka, A. M. Wodtke, T. Schäfer, Vibrational Relaxation of Highly Vibrationally Excited CO Scattered from $\mathrm{Au}(111)$ : Evidence for $\mathrm{CO}^{-}$Formation, J. Phys. Chem. Lett. 2017, 8, 4887-4892, https://doi.org/10.1021/acs.jpclett.7b02207.

[32] R. J. V. Wagner, B. C. Krüger, G. B. Park, M. Wallrabe, A. M. Wodtke, T. Schäfer, Electron Transfer Mediates Vibrational Relaxation of CO in Collisions with $\operatorname{Ag}(111)$, Phys. Chem. Chem. Phys. 2019, 21, 1650-1655, https: //doi.org/10.1039/C8CP06041J.

[33] I. Rahinov, R. Cooper, D. Matsiev, C. Bartels, D. J. Auerbach, A. M. Wodtke, Quantifying the Breakdown of the Born-Oppenheimer Approximation in Surface Chemistry, Phys. Chem. Chem. Phys. 2011, 13, 12680-12692, http://dx.doi.org/ 10.1039/C1CP20356H.

[34] K. Golibrzuch, N. Bartels, D. J. Auerbach, A. M. Wodtke, The Dynamics of Molecular Interactions and Chemical Reactions at Metal Surfaces: Testing the Foundations of Theory, Annu. Rev. Phys. Chem. 2015, 66, 399-425, https://doi. org/10.1146/annurev-physchem-040214-121958.

[35] H. G. Liddell, R. Scott, A Greek-English Lexicon, $9^{\text {th }}$ edition with a revised supplement, Clarendon Press, Oxford University Press, New York, 1996.

[36] L. J. Butler, Chemical Reaction Dynamics beyond the Born-Oppenheimer Approximation, Annu. Rev. Phys. Chem. 1998, 49, 125-171, https://doi.org/10. 1146/annurev.physchem.49.1.125.

[37] A. W. Jasper, C. Zhu, S. Nangia, D. G. Truhlar, Introductory Lecture: Nonadiabatic Effect in Chemical Dynamics, Faraday Discuss. 2004, 127, 1-22, https: //doi.org/10.1039/B405601A.

[38] A. W. Jasper, S. Nangia, C. Zhu, D. G. Truhlar, Non-Born-Oppenheimer Molecular Dynamics, Acc. Chem. Res. 2006, 39, 101-108, https://doi.org/10.1021/ ar040206v.

[39] J. C. Tully, Nonadiabatic Processes in Molecular Collisions, in Modern Theoretical Chemistry, Volume 2, Dynamics of Molecular Collisions, Part B, edited by W. H. Miller, Springer Science+Business Media, New York, pp. 217-267, 1976, https: //doi.org/10.1007/978-1-4757-0644-4_5. 
[40] J. C. Tully, Perspective on "Zur Quantentheorie der Molekeln", Theor. Chem. Acc. 2000, 103, 173-176, https://doi.org/10.1007/s002149900049.

[41] T. S. Rose, M. J. Rosker, A. H. Zewail, Femtosecond Real-Time Observation of Wave Packet Oscillations (Resonance) in Dissociation Reactions, J. Chem. Phys. 1988, 88, 6672-6673, https://doi.org/10.1063/1.454408.

[42] H. S. W. Massey, Collisions Between Atoms and Molecules at Ordinary Temperatures, Rep. Prog. Phys. 1949, 12, 248, http://stacks.iop.org/0034-4885/12/i=1/ $\mathrm{a}=311$.

[43] C. Zener, R. H. Fowler, Non-Adiabatic Crossing of Energy Levels, Proc. R. Soc. London A 1932, 137, 696-702, https://doi.org/10.1098/rspa.1932.0165.

[44] E. E. Nikitin, Nonadiabatic Transitions: What We Learned from Old Masters and How Much We Owe Them, Annu. Rev. Phys. Chem. 1999, 50, 1-21, https: //doi.org/10.1146/annurev.physchem.50.1.1.

[45] A. M. Wodtke, D. Matsiev, D. J. Auerbach, Energy Transfer and Chemical Dynamics at Solid Surfaces: The Special Role of Charge Transfer, Prog. Surf. Sci. 2008, 83, 167-214, https://doi.org/10.1016/j.progsurf.2008.02.001.

[46] J. C. Tully, Theories of the Dynamics of Inelastic and Reactive Processes at Surfaces, Ann. Rev. Phys. Chem. 1980, 31, 319-343, https://doi.org/10.1146/ annurev.pc.31.100180.001535.

[47] J. C. Tully, Chemical Dynamics at Metal Surfaces, Ann. Rev. Phys. Chem. 2000, 51, 153-178, https://doi.org/10.1146/annurev.physchem.51.1.153.

[48] G.-J. Kroes, Frontiers in Surface Scattering Simulations, Science 2008, 321, 794797, https://doi.org/10.1126/science.1157717.

[49] H. Arnolds, Vibrational Dynamics of Adsorbates - Quo Vadis?, Prog. Surf. Sci. 2011, 86, 1-40, https://doi.org/10.1016/j.progsurf.2010.10.001.

[50] G. E. Ewing, Energy Flow from Excited Molecules on Salt Surfaces, Acc. Chem. Res. 1992, 25, 292-299, https://doi.org/10.1021/ar00019a004.

[51] R. J. Maurer, M. Askerka, V. S. Batista, J. C. Tully, Ab Initio Tensorial Electronic Friction for Molecules on Metal Surfaces: Nonadiabatic Vibrational Relaxation, Phys. Rev. B 2016, 94, 115432, https://doi.org/10.1103/PhysRevB.94.115432. 
Bibliography

[52] C. Frischkorn, M. Wolf, Femtochemistry at Metal Surfaces: Nonadiabatic Reaction Dynamics, Chem. Rev. 2006, 106, 4207-4233, https://doi.org/10.1021/ cr050161r.

[53] T. Greber, Charge-Transfer Induced Particle Emission in Gas Surface Reactions, Surf. Sci. Rep. 1997, 28, 1-64, https://doi.org/10.1016/S0167-5729(97)00005-8.

[54] E. Hasselbrink, Nonadiabatic Pathways in the Dissociative Adsorption of Simple Molecules, Isr. J. Chem. 2005, 45, 37-44, https://doi.org/10.1560/ YQKH-DTXV-7M69-BK0L.

[55] H. Nienhaus, Electronic Excitations by Chemical Reactions on Metal Surfaces, Surf. Sci. Rep. 2002, 45, 1-78, https://doi.org/10.1016/S0167-5729(01)00019-X.

[56] E. Hasselbrink, Non-Adiabaticity in Surface Chemical Reactions, Surf. Sci. 2009, 603, 1564-1570, https://doi.org/10.1016/j.susc.2008.12.037.

[57] D. Diesing, E. Hasselbrink, Chemical Energy Dissipation at Surfaces under UHV and High Pressure Conditions Studied Using Metal-Insulator-Metal and Similar Devices, Chem. Soc. Rev. 2016, 45, 3747-3755, https://doi.org/10.1039/ c5cs00932d.

[58] J. Barker, D. Auerbach, Gas-Surface Interactions and Dynamics; Thermal Energy Atomic and Molecular Beam Studies, Surf. Sci. Rep. 1984, 4, 1-99, https://doi. org/10.1016/0167-5729(84)90005-0.

[59] C. T. Rettner, D. J. Auerbach, J. C. Tully, A. W. Kleyn, Chemical Dynamics at the Gas-Surface Interface, J. Chem. Phys. 1996, 100, 13021-13033, https: //doi.org/10.1021/jp9536007.

[60] H. Chadwick, R. D. Beck, Quantum State Resolved Gas-Surface Reaction Dynamics Experiments: A Tutorial Review, Chem. Soc. Rev. 2016, 45, 3576-3594, https://doi.org/10.1039/c5cs00476d.

[61] A. M. Wodtke, Electronically Non-Adiabatic Influences in Surface Chemistry and Dynamics, Chem. Soc. Rev. 2016, 45, 3641-3657, http://dx.doi.org/10.1039/ C6CS00078A.

[62] C. T. Rettner, F. Fabre, J. Kimman, D. J. Auerbach, Observation of Direct Vibrational Excitation in Gas-Surface Collisions: NO on $\mathrm{Ag}(111)$, Phys. Rev. Lett. 1985, 55, 1904-1907, https://doi.org/10.1103/PhysRevLett.55.1904. 
[63] C. Rettner, J. Kimman, F. Fabre, D. Auerbach, H. Morawitz, Direct Vibrational Excitation in Gas-Surface Collisions of NO with $\mathrm{Ag}(111)$, Surf. Sci. 1987, 192, 107-130, https://doi.org/10.1016/S0039-6028(87)81165-2.

[64] J. W. Gadzuk, Vibrational Excitation in Molecule-Surface Collisions due to Temporary Negative Molecular Ion Formation, J. Chem. Phys. 1983, 79, 6341-6348, https://doi.org/10.1063/1.445742.

[65] D. Newns, Electron-Hole Pair Mechanism for Excitation of Intramolecular Vibrations in Molecule-Surface Scattering, Surf. Sci. 1986, 171, 600-614, https: //doi.org/10.1016/0039-6028(86)91062-9.

[66] D. Matsiev, Z. Li, R. Cooper, I. Rahinov, C. Bartels, D. J. Auerbach, A. M. Wodtke, On the Temperature Dependence of Electronically Non-Adiabatic Vibrational Energy Transfer in Molecule-Surface Collisions, Phys. Chem. Chem. Phys. 2011, 13, 8153-8162, https://doi.org/10.1039/c0cp01418d.

[67] B. D. Kay, T. D. Raymond, M. E. Coltrin, Observation of Direct Multiquantum Vibrational Excitation in Gas-Surface Scattering: $\mathrm{NH}_{3}$ on $\mathrm{Au}(111)$, Phys. Rev. Lett. 1987, 59, 2792-2794, https://doi.org/10.1103/PhysRevLett.59.2792.

[68] E. K. Watts, J. L. Siders, G. O. Sitz, Vibrational Excitation of NO Scattered from Cu(110), Surf. Sci. 1997, 374, 191-196, https://doi.org/10.1016/S0039-6028(96) 01194-6.

[69] R. Cooper, I. Rahinov, Z. Li, D. Matsiev, D. J. Auerbach, A. M. Wodtke, Vibrational Overtone Excitation in Electron Mediated Energy Transfer at Metal Surfaces, Chem. Sci. 2010, 1, 55-61, https://doi.org/10.1039/c0sc00141d.

[70] R. Cooper, Z. Li, K. Golibrzuch, C. Bartels, I. Rahinov, D. J. Auerbach, A. M. Wodtke, On the Determination of Absolute Vibrational Excitation Probabilities in Molecule-Surface Scattering: Case Study of NO on $\mathrm{Au}(111)$, J. Chem. Phys. 2012, 137, 064705, https://doi.org/10.1063/1.4738596.

[71] R. Cooper, C. Bartels, A. Kandratsenka, I. Rahinov, N. Shenvi, K. Golibrzuch, Z. Li, D. J. Auerbach, J. C. Tully, A. M. Wodtke, Multiquantum Vibrational Excitation of NO Scattered from $\mathrm{Au}(111)$ : Quantitative Comparison of Benchmark Data to Ab Initio Theories of Nonadiabatic Molecule-Surface Interactions, Angew. Chem. Int. Ed. 2012, 124, 5038-5042, https://doi.org/10.1002/ange.201201168. 
Bibliography

[72] K. Golibrzuch, A. Kandratsenka, I. Rahinov, R. Cooper, D. J. Auerbach, A. M. Wodtke, C. Bartels, Experimental and Theoretical Study of Multi-Quantum Vibrational Excitation: $\mathrm{NO}(v=0 \rightarrow 1,2,3)$ in Collisions with $\mathrm{Au}(111), J$. Phys. Chem. A 2013, 117, 7091-7101, https://doi.org/10.1021/jp400313b.

[73] Q. Ran, D. Matsiev, D. J. Auerbach, A. M. Wodtke, Observation of a Change of Vibrational Excitation Mechanism with Surface Temperature: HCl Collisions with $\mathrm{Au}(111)$, Phys. Rev. Lett. 2007, 98, 237601, https://doi.org/10.1103/ PhysRevLett.98.237601.

[74] J. Geweke, P. R. Shirhatti, I. Rahinov, C. Bartels, A. M. Wodtke, Vibrational Energy Transfer Near a Dissociative Adsorption Transition State: State-to-State Study of $\mathrm{HCl}$ Collisions at $\mathrm{Au}(111)$, J. Chem. Phys. 2016, 145, 054709, https: //doi.org/10.1063/1.4959968.

[75] C. T. Rettner, The Search for Direct Vibrational Excitation in Gas-Surface Collisions of $\mathrm{CO}$ with $\mathrm{Au}(111)$, J. Chem. Phys. 1993, 99, 5481-5489, https: //doi.org/10.1063/1.465965.

[76] T. Schäfer, N. Bartels, K. Golibrzuch, C. Bartels, H. Köckert, D. J. Auerbach, T. N. Kitsopoulos, A. M. Wodtke, Observation of Direct Vibrational Excitation in Gas-Surface Collisions of CO with $\mathrm{Au}(111)$ : A New Model System for Surface Dynamics, Phys. Chem. Chem. Phys. 2013, 15, 1863-1867, https://doi.org/10. 1039/c2cp43351f.

[77] P. R. Shirhatti, J. Werdecker, K. Golibrzuch, A. M. Wodtke, C. Bartels, Electron Hole Pair Mediated Vibrational Excitation in CO Scattering from $\mathrm{Au}(111)$ : Incidence Energy and Surface Temperature Dependence, J. Chem. Phys. 2014, 141, 124704, https://doi.org/10.1063/1.4894814.

[78] J. Werdecker, P. R. Shirhatti, K. Golibrzuch, C. Bartels, A. M. Wodtke, D. J. Harding, Electronically Nonadiabatic Vibrational Excitation of $\mathrm{N}_{2}$ Scattered from Pt(111), J. Phys. Chem. C 2015, 119, 14722-14727, https://doi.org/10.1021/acs. jpcc.5b00202.

[79] Y. Huang, A. M. Wodtke, H. Hou, C. T. Rettner, D. J. Auerbach, Observation of Vibrational Excitation and Deexcitation for NO $(v=2)$ Scattering from $\mathrm{Au}(111)$ : Evidence for Electron-Hole-Pair Mediated Energy Transfer, Phys. Rev. Lett. 2000, 84, 2985-2988, https://doi.org/10.1103/PhysRevLett.84.2985. 
[80] K. Golibrzuch, P. R. Shirhatti, J. Altschäffel, I. Rahinov, D. J. Auerbach, A. M. Wodtke, C. Bartels, State-to-State Time-of-Flight Measurements of NO Scattering from $\mathrm{Au}(111)$ : Direct Observation of Translation-to-Vibration Coupling in Electronically Nonadiabatic Energy Transfer, J. Phys. Chem. A 2013, 117, 87508760, https://doi.org/10.1021/jp403382b.

[81] K. Golibrzuch, P. R. Shirhatti, I. Rahinov, D. J. Auerbach, A. M. Wodtke, C. Bartels, Incidence Energy Dependent State-to-State Time-of-Flight Measurements of $\operatorname{NO}(v=3)$ Collisions with $\mathrm{Au}(111)$ : The Fate of Incidence Vibrational and Translational Energy, Phys. Chem. Chem. Phys. 2014, 16, 7602-7610, http://dx.doi.org/10.1039/C3CP55224A.

[82] K. Golibrzuch, P. R. Shirhatti, I. Rahinov, A. Kandratsenka, D. J. Auerbach, A. M. Wodtke, C. Bartels, The Importance of Accurate Adiabatic Interaction Potentials for the Correct Description of Electronically Nonadiabatic Vibrational Energy Transfer: A Combined Experimental and Theoretical Study of $\mathrm{NO}(v=3)$ Collisions with a $\mathrm{Au}(111)$ Surface, J. Chem. Phys. 2014, 140, 044701, https: //doi.org/10.1063/1.4861660.

[83] A. M. Wodtke, Y. Huang, D. J. Auerbach, Interaction of $\mathrm{NO}(v=12)$ with LiF(001): Evidence for Anomalously Large Vibrational Relaxation Rates, $J$. Chem. Phys. 2003, 118, 8033-8041, https://doi.org/10.1063/1.1560143.

[84] N. Bartels, K. Golibrzuch, C. Bartels, L. Chen, D. J. Auerbach, A. M. Wodtke, T. Schäfer, Observation of Orientation-Dpendent Electron Transfer in MoleculeSurface Collisions, Proc. Natl. Acad. Sci. 2013, 110, 17738-17743, https://doi. org/10.1073/pnas.1312200110.

[85] N. Bartels, K. Golibrzuch, C. Bartels, L. Chen, D. J. Auerbach, A. M. Wodtke, T. Schäfer, Dynamical Steering in an Electron Transfer Surface Reaction: Oriented $\mathrm{NO}\left(v=3,0.08<E_{\mathrm{i}}<0.89 \mathrm{eV}\right)$ Relaxation in Collisions With a $\mathrm{Au}(111)$ Surface, J. Chem. Phys. 2014, 140, 054710, https://doi.org/10.1063/1.4863862.

[86] N. Bartels, B. C. Krüger, D. J. Auerbach, A. M. Wodtke, T. Schäfer, Controlling an Electron-Transfer Reaction at a Metal Surface by Manipulating Reactant Motion and Orientation, Angew. Chem. Int. Ed. 2014, 53, 13690-13694, https://doi.org/10.1002/anie.201407051.

[87] B. C. Krüger, N. Bartels, A. M. Wodtke, T. Schäfer, Final Rotational State Distributions from $\mathrm{NO}\left(v_{\mathrm{i}}=11\right)$ in Collisions with $\mathrm{Au}(111)$ : The Magnitude of 
Bibliography

Vibrational Energy Transfer Depends on Orientation in Molecule-Surface Collisions, Phys. Chem. Chem. Phys. 2016, 18, 14976-14979, https://doi.org/10.1039/ c6cp02100j.

[88] J. D. White, J. Chen, D. Matsiev, D. J. Auerbach, A. M. Wodtke, Conversion of Large-Amplitude Vibration to Electron Excitation at a Metal Surface, Nature 2005, 433, 503-505, http://dx.doi.org/10.1038/nature03213.

[89] J. D. White, J. Chen, D. Matsiev, D. J. Auerbach, A. M. Wodtke, Vibrationally Promoted Electron Emission from Low Work-Function Metal Surfaces, J. Chem. Phys. 2006, 124, 064702, https://doi.org/10.1063/1.2166360.

[90] J. L. LaRue, J. D. White, N. H. Nahler, Z. Liu, Y. Sun, P. A. Pianetta, D. J. Auerbach, A. M. Wodtke, The Work Function of Submonolayer Cesium-Covered Gold: A Photoelectron Spectroscopy Study, J. Chem. Phys. 2008, 129, 024709, https://doi.org/10.1063/1.2953712.

[91] N. H. Nahler, A. M. Wodtke, Dynamics of Molecule-Induced Electron Emission from Surfaces, Mol. Phys. 2008, 106, 2227-2244, https://doi.org/10.1080/ 00268970802418955.

[92] J. LaRue, T. Schäfer, D. Matsiev, L. Velarde, N. H. Nahler, D. J. Auerbach, A. M. Wodtke, Vibrationally Promoted Electron Emission at a Metal Surface: Electron Kinetic Energy Distributions, Phys. Chem. Chem. Phys. 2011, 13, 9799, http://dx.doi.org/10.1039/C0CP01626H.

[93] J. L. LaRue, T. Schäfer, D. Matsiev, L. Velarde, N. H. Nahler, D. J. Auerbach, A. M. Wodtke, Electron Kinetic Energies from Vibrationally Promoted Surface Exoemission: Evidence for a Vibrational Autodetachment Mechanism, J. Phys. Chem. A 2011, 115, 14306-14314, https://doi.org/10.1021/jp205868g.

[94] G. Miao, W. Dou, J. Subotnik, Vibrational Relaxation at a Metal Surface: Electronic Friction versus Classical Master Equations, J. Chem. Phys. 2017, 147, 224105, https://doi.org/10.1063/1.5000237.

[95] S. P. Rittmeyer, J. Meyer, J. I. n. Juaristi, K. Reuter, Electronic Friction-Based Vibrational Lifetimes of Molecular Adsorbates: Beyond the Independent-Atom Approximation, Phys. Rev. Lett. 2015, 115, 046102, https://doi.org/10.1103/ PhysRevLett.115.046102. 
[96] M. Alducin, R. D. M. no, J. I. Juaristi, Non-Adiabatic Effects in Elementary Reaction Processes at Metal Surfaces, Prog. Surf. Sci. 2017, 92, 317-340, https: //doi.org/10.1016/j.progsurf.2017.09.002.

[97] P. Spiering, J. Meyer, Testing Electronic Friction Models: Vibrational Deexcitation in Scattering of $\mathrm{H}_{2}$ and $\mathrm{D}_{2}$ from $\mathrm{Cu}(111)$, J. Phys. Chem. Lett. 2018, 9, 1803-1808, https://doi.org/10.1021/acs.jpclett.7b03182.

[98] S. Li, H. Guo, Monte Carlo Wave Packet Study of Negative Ion Mediated Vibrationally Inelastic Scattering of NO From the Metal Surface, J. Chem. Phys. 2002, 117, 4499-4508, https://doi.org/10.1063/1.1498479.

[99] G. Herzberg, I. Spectra of Diatomic Molecules, in Molecular Spectra and Molecular Structure, $2^{\text {nd }}$ edition, Van Nostrand Reinhold Company, New York, 1950.

[100] J. L. Dunham, The Energy Levels of a Rotating Vibrator, Phys. Rev. 1932, 41, 721-731, https://doi.org/10.1103/PhysRev.41.721.

[101] P. R. Bunker, P. Jensen, Molecular Symmetry and Spectroscopy, $2^{\text {nd }}$ edition, NRC Research Press, Ottawa, 1998.

[102] M. Schneider, K. Evenson, M. Vanek, D. Jennings, J. Wells, A. Stahn, W. Urban, Heterodyne Frequency Measurements of ${ }^{12} \mathrm{C}^{16} \mathrm{O}$ Laser Transitions, J. Mol. Spectrosc. 1989, 135(2), 197-206, https://doi.org/10.1016/0022-2852(89)90150-1.

[103] J. Simmons, A. Bass, S. Tilford, The Fourth Positive System of Carbon Monoxide Observed in Absorption at High Resolution in the Vacuum Ultraviolet Region, Astrophys. J. 1969, 155, 345-358, https://doi.org/10.1086/149869.

[104] F. Hund, Zur Deutung einiger Erscheinungen in den Molekelspektren, Z. Phys. 1926, 36, 657-674, https://doi.org/10.1007/BF01400155.

[105] T. C. James, Transition Moments, Franck-Condon Factors, and Lifetimes of Forbidden Transitions. Calculation of the Intensity of the Cameron System of CO, J. Chem. Phys. 1971, 55, 4118-4124, https://doi.org/10.1063/1.1676710.

[106] N. Bartels, T. Schäfer, J. Hühnert, R. W. Field, A. M. Wodtke, Production of a Beam of Highly Vibrationally Excited CO Using Perturbations, J. Chem. Phys. 2012, 136, 214201, https://doi.org/10.1063/1.4722090.

[107] S. Y. T. van de Meerakker, H. L. Bethlem, G. Meijer, Taming Molecular Beams, Nature Phys. 2008, 4, 595-602, https://doi.org/10.1038/nphys1031. 
Bibliography

[108] M. Schnell, G. Meijer, Cold Molecules: Preparation, Applications, and Challenges, Angew. Chem. Int. Ed. 2009, 48, 6010-6031, https://doi.org/10.1002/ anie.200805503.

[109] J. Stark, Beobachtungen über den Effekt des elektrischen Feldes auf Spektrallinien, Sitzungsberichte der Königlich Preussischen Akademie der Wissenschaften 1913, 932-946.

[110] C. A. Burrus, Stark Effect from 1.1 to 2.6 Millimeters Wavelength: $\mathrm{PH}_{3}, \mathrm{PD}_{3}$, DI, and CO, J. Chem. Phys. 1958, 28, 427-429, https://doi.org/10.1063/1.1744151.

[111] B. G. Wicke, R. W. Field, W. Klemperer, Fine Structure, Dipole Moment, and Perturbation Analysis of $\mathrm{a}^{3} \Pi$ CO, J. Chem. Phys. 1972, 56, 5758-5770, https: //doi.org/10.1063/1.1677113.

[112] J. J. Gilijamse, S. Hoekstra, S. A. Meek, M. Metsälä, S. Y. T. van de Meerakker, G. Meijer, G. C. Groenenboom, The Radiative Lifetime of Metastable CO (a $\left.{ }^{3} \Pi, v=0\right)$, J. Chem. Phys. 2007, 127, 221102, https://doi.org/10.1063/1. 2813888.

[113] R. J. V. Wagner, Surface Scattering Using Velocity Controlled Molecular Beams of Metastable Carbon Monoxide, Master's Thesis, Georg-August-Universität Göttingen, Göttingen, 2013.

[114] J. M. Brown, A. Carrington, Rotational Spectroscopy of Diatomic Molecules, Cambridge Molecular Science, Cambridge University Press, Cambridge, 2003, https://doi.org/10.1017/CBO9780511814808.

[115] S. A. Meek, A Stark Decelerator on a Chip, Dissertation, Freie Universität Berlin, Fritz-Haber-Institut der Max-Planck-Gesellschaft, Berlin, 2010, https: //refubium.fu-berlin.de/handle/fub188/13291.

[116] R. W. Field, S. G. Tilford, R. A. Howard, J. D. Simmons, Fine Structure and Perturbation Analysis of the $\mathrm{a}^{3} \Pi$ State of CO, J. Mol. Spectrosc. 1972, 44, 347382, https://doi.org/10.1016/0022-2852(72)90110-5.

[117] R. T. Jongma, T. Rasing, G. Meijer, Two-Dimensional Imaging of Metastable CO Molecules, J. Chem. Phys. 1995, 102, 1925-1933, https://doi.org/10.1063/1. 468758 . 
[118] J. P. Gordon, H. J. Zeiger, C. H. Townes, Molecular Microwave Oscillator and New Hyperfine Structure in the Microwave Spectrum of $\mathrm{NH}_{3}$, Phys. Rev. 1954, 95, 282-284, https://doi.org/10.1103/PhysRev.95.282.

[119] J. P. Gordon, H. J. Zeiger, C. H. Townes, The Maser-New Type of Microwave Amplifier, Frequency Standard, and Spectrometer, Phys. Rev. 1955, 99, 12641274, https://doi.org/10.1103/PhysRev.99.1264.

[120] H. G. Bennewitz, W. Paul, C. Schlier, Fokussierung polarer Moleküle, Z. Phys. 1955, 141, 6-15, https://doi.org/10.1007/BF01327279.

[121] J. R. Zacharias, J. G. King, Linear Decelerator for Molecules, in: Atomic Beams, Research Laboratory of Electronics (RLE) at the Massachusetts Institute of Technology (MIT), January 15 ${ }^{\text {th }}$, Quarterly Progress Report 1958, 48(7), 56-57, http://hdl.handle.net/1721.1/52129.

[122] J. G. King, Experiments with Slow Molecules, $13^{\text {th }}$ Annual Symposium on Frequency Control 1959, 603, https://doi.org/10.1109/FREQ.1959.199414.

[123] D. Auerbach, E. E. A. Bromberg, L. Wharton, Alternate-Gradient Focusing of Molecular Beams, J. Chem. Phys. 1966, 45, 2160-2166, https://doi.org/10.1063/ 1.1727902 .

[124] R. Golub, On Decelerating Molecules, Dissertation, Massachusetts Institute of Technology, Cambridge, USA, 1967.

[125] S. A. Meek, H. Conrad, G. Meijer, A Stark Decelerator on a Chip, New J. Phys. 2009, 11, 055024, https://doi.org/10.1088/1367-2630/11/5/055024.

[126] D. P. Engelhart, F. Grätz, R. J. V. Wagner, H. Haak, G. Meijer, A. M. Wodtke, T. Schäfer, A New Stark Decelerator Based Surface Scattering Instrument for Studying Energy Transfer at the Gas-Surface Interface, Rev. Sci. Instrum. 2015, 86, 043306, https://doi.org/10.1063/1.4918797.

[127] F. Grätz, Molecule-Surface Scattering with Velocity-Controlled Molecular Beams, Dissertation, Freie Universität Berlin, Fritz-Haber-Institut der Max-PlanckGesellschaft, Berlin, 2014, https://refubium.fu-berlin.de/handle/fub188/1770.

[128] T. Schäfer, N. Bartels, N. Hocke, X. Yang, A. M. Wodtke, Orienting Polar Molecules Without Hexapoles: Optical State Selection with Adiabatic Orientation, Chem. Phys. Lett. 2012, 535, 1-11, https://doi.org/10.1016/j.cplett.2012.03.056. 
Bibliography

[129] B. C. Krüger, From Diatomic to Polyatomic Quantum-State-Resolved MoleculeSurface Scattering, Dissertation, Georg-August-Universität Göttingen, Göttingen, 2017, http://hdl.handle.net/11858/00-1735-0000-0023-3F1E-7.

[130] U. Even, J. Jortner, D. Noy, N. Lavie, C. Cossart-Magos, Cooling of Large Molecules Below $1 \mathrm{~K}$ and He Clusters Formation, J. Chem. Phys. 2000, 112, 80688071, https://doi.org/10.1063/1.481405.

[131] D. R. Miller, Free Jet Sources, in Atomic and Molecular Beam Methods, Volume 1, edited by G. Scoles, Oxford University Press, New York, Oxford, 1988.

[132] Handbook of Auger Electron Spectroscopy, $2^{\text {nd }}$ edition, edited by L. E. Davis, N. C. MacDonald, P. W. Palmberg, G. E. Riach, R. E. Weber, Physical Electronics Industries, Inc., Eden Prairie, Minnesota, USA, 1976.

[133] R. Musket, W. McLean, C. Colmenares, D. Makowiecki, W. Siekhaus, Preparation of Atomically Clean Surfaces of Selected Elements: A Review, Appl. Surf. Sci. 1982, 10, 143-207, https://doi.org/10.1016/0378-5963(82)90142-8.

[134] K. Golibrzuch, Quantum-State Specific Scattering of Molecules from Surfaces, Dissertation, Georg-August-Universität Göttingen, Göttingen, 2014, http://hdl. handle.net/11858/00-1735-0000-0023-9904-3.

[135] D. P. Engelhart, Electron Emission from Metastable Carbon Monoxide Molecules at Adsorbate Covered Au(111) Surfaces, Dissertation, GeorgAugust-Universität Göttingen, Göttingen, 2015, http://hdl.handle.net/11858/ 00-1735-0000-0022-604F-4.

[136] S. N. Vogels, Z. Gao, S. Y. T. van de Meerakker, Optimal Beam Sources for Stark Decelerators in Collision Experiments: A Tutorial Review, EPJ Techniques and Instrumentation 2015, 2:12, https://doi.org/10.1140/epjti/s40485-015-0021-y.

[137] J. Küpper, F. Filsinger, F. Grätz, S. Putzke, Y.-P. Chang, libcoldmol: A Particle Trajectory Calculation Framework, 2003-2013.

[138] J. H. Blokland, J. Riedel, S. Putzke, B. G. Sartakov, G. C. Groenenboom, G. Meijer, Producing Translationally Cold, Ground-Sate CO Molecules, J. Chem. Phys. 2011, 135, 114201, https://doi.org/10.1063/1.3637037.

[139] L. Velarde, D. P. Engelhart, D. Matsiev, J. LaRue, D. J. Auerbach, A. M. Wodtke, Generation of Tunable Narrow Bandwidth Nanosecond Pulses in the Deep Ultra- 
violet for Efficient Optical Pumping and High Resolution Spectroscopy, Rev. Sci. Instrum. 2010, 81, 063106, https://doi.org/10.1063/1.3436973.

[140] X. Yang, A. M. Wodtke, Efficient State-Specific Preparation of Highly Vibrationally Excited $\mathrm{NO}\left(\mathrm{X}^{2} \Pi\right)$, J. Chem. Phys. 1990, 92, 116-120, https://doi.org/10. $1063 / 1.458480$.

[141] J. Price, J. Mack, C. Rogaski, A. Wodtke, Vibrational-State-Specific SelfRelaxation Rate Constant. Measurements of Highly Vibrationally Excited $\mathrm{O}_{2}(v=$ 19-28), Chem. Phys. 1993, 175, 83-98, https://doi.org/10.1016/0301-0104(93) 80230-7.

[142] G. Berden, R. T. Jongma, D. van der Zande, G. Meijer, A Reanalysis of the $\mathrm{k}^{3} \Pi$ State of CO, J. Chem. Phys. 1997, 107, 8303-8310, https://doi.org/10.1063/1. 475031.

[143] M. Bowker, K. Waugh, The Adsorption of Chlorine and Chloridation of $\operatorname{Ag}(111)$, Surf. Sci. 1983, 134, 639-664, https://doi.org/10.1016/0039-6028(83)90063-8.

[144] K. Wu, D. Wang, J. Deng, X. Wei, Y. Cao, M. Zei, R. Zhai, X. Guo, Chlorine on $\operatorname{Ag}(111)$ : The Intermediate Coverage Case, Surf. Sci. 1992, 264, 249-259, https://doi.org/10.1016/0039-6028(92)90182-6.

[145] E. R. Frank, R. J. Hamers, Chlorine-Induced Restructuring of Ag(111) Films Observed by Scanning Tunneling Microscopy, J. Catal. 1997, 172, 406-413, https: //doi.org/10.1006/jcat.1997.1798.

[146] B. Andryushechkin, K. Eltsov, V. Shevlyuga, V. Yurov, Direct STM Observation of Surface Modification and Growth of $\mathrm{AgCl}$ Islands on $\mathrm{Ag}(111)$ upon Chlorination at Room Temperature, Surf. Sci. 1999, 431, 96-108, https://doi.org/10.1016/ S0039-6028(99)00429-X.

[147] H. Piao, K. Adib, M. A. Barteau, A Temperature-Programmed X-Ray Photoelectron Spectroscopy (TPXPS) Study of Chlorine Adsorption and Diffusion on $\operatorname{Ag}(111)$, Surf. Sci. 2004, 557, 13-20, https://doi.org/10.1016/j.susc.2004.03.063.

[148] S. R. Bare, K. Griffiths, W. Lennard, H. Tang, Generation of Atomic Oxygen on $\mathrm{Ag}(111)$ and $\mathrm{Ag}(110)$ Using $\mathrm{NO}_{2}$ : a TPD, LEED, HREELS, XPS and NRA Study, Surf. Sci. 1995, 342, 185-198, https://doi.org/10.1016/0039-6028(95)00670-2. 
Bibliography

[149] W. Huang, J. White, Revisiting $\mathrm{NO}_{2}$ on $\mathrm{Ag}(111)$ : A Detailed TPD and RAIRS Study, Surf. Sci. 2003, 529, 455-470, https://doi.org/10.1016/S0039-6028(03) 00332-7.

[150] S. Günther, S. Böcklein, J. Wintterlin, M. A. Niño, T. O. Menteş, A. Locatelli, Locating Catalytically Active Oxygen on $\mathrm{Ag}(111)$ - Spectromicroscopy Study, Chem. Cat. Chem. 2013, 5, 3342-3350, https://doi.org/10.1002/cctc.201300355.

[151] D. J. Auerbach, Velocity Measurements by Time-of-Flight Methods, in Atomic and Molecular Beam Methods, Volume 1, edited by G. Scoles, Oxford University Press, New York, Oxford, 1988.

[152] R. J. Le Roy, RKR1 2.0, A Computer Program Implementing the First-Order RKR Method for Determining Diatomic Molecule Potential Energy Functions, University of Waterloo, (CP657R), 2004.

[153] R. J. Le Roy, LEVEL 8.0, A Computer Program for Solving the Radial Schrödinger Equation for Bound and Quasibound Levels, University of Waterloo, (CP-663), 2007.

[154] C. M. Western, PGOPHER, A Program for Simulating Rotational, Vibrational and Electronic Spectra, Version: 10.0.505.0, University of Bristol, http://pgopher.chm.bris.ac.uk.

[155] C. M. Western, PGOPHER: A Program for Simulating Rotational, Vibrational and Electronic Spectra, J. Quant. Spectrosc. Radiat. Transfer 2017, 186, 221-242, https://doi.org/10.1016/j.jqsrt.2016.04.010.

[156] L. Gerö, Über $\mathrm{A}^{1} \Pi \rightarrow \mathrm{X}^{1} \Sigma$-(IV. Pos.) Kohleoxydbanden, Z. Phys. 1936, 99, 52-64, https://doi.org/10.1007/BF01847813.

[157] W. Demtröder, Laserspektroskopie 1, 6., aktualisierte Auflage, Springer, Heidelberg, 2011.

[158] N. Bartels, B. C. Krüger, S. Meyer, A. M. Wodtke, T. Schäfer, Suppression of Spontaneous Emission in the Optical Pumping of Molecules: Pump-DumpSweep-Probe, J. Phys. Chem. Lett. 2013, 4, 2367-2370, https://doi.org/10.1021/ jz401266m.

[159] R. M. Logan, R. E. Stickney, Simple Classical Model for the Scattering of Gas Atoms from a Solid Surface, J. Chem. Phys. 1966, 44, 195-201, https://doi.org/ 10.1063/1.1726446. 
[160] B. Baule, Theoretische Behandlung der Erscheinungen in verdünnten Gasen, Ann. Phys. 1914, 349, 145-176, http://dx.doi.org/10.1002/andp.19143490908.

[161] C. T. Rettner, J. Kimman, D. J. Auerbach, Inelastic Scattering of NO from $\mathrm{Ag}(111)$ : Internal State, Angle, and Velocity Resolved Measurements, J. Chem. Phys. 1991, 94, 734-750, https://doi.org/10.1063/1.460342.

[162] J. Kimman, C. T. Rettner, D. J. Auerbach, J. A. Barker, J. C. Tully, Correlation Between Kinetic-Energy Transfer to Rotation and to Phonons in Gas-Surface Collisions of NO with $\mathrm{Ag}(111)$, Phys. Rev. Lett. 1986, 57, 2053-2056, https: //doi.org/10.1103/PhysRevLett.57.2053.

[163] K. R. Lykke, B. D. Kay, Rotationally Inelastic Gas-Surface Scattering: HCl from $\mathrm{Au}(111)$, J. Chem. Phys. 1990, 92, 2614-2623, https://doi.org/10.1063/1.457955.

[164] M. A. Hines, R. N. Zare, The Interaction of CO with Ni(111): Rainbows and Rotational Trapping, J. Chem. Phys. 1993, 98, 9134-9147, https://doi.org/10. 1063/1.464421.

[165] E. Watts, G. O. Sitz, State-to-State Scattering in a Reactive System: $\mathrm{H}_{2}(v=1$, $J=1$ ) from $\mathrm{Cu}(100), J$. Chem. Phys. 2001, 114, 4171-4179, https://doi.org/10. $1063 / 1.1344233$.

[166] I. Rahinov, R. Cooper, C. Yuan, X. Yang, D. J. Auerbach, A. M. Wodtke, Efficient Vibrational and Translational Excitations of a Solid Metal Surface: State-to-State Time-of-Flight Measurements of $\mathrm{HCl}(v=2, J=1)$ Scattering from $\mathrm{Au}(111), J$. Chem. Phys. 2008, 129, 214708, https://doi.org/10.1063/1.3028542.

[167] J. E. Hurst, C. A. Becker, J. P. Cowin, K. C. Janda, L. Wharton, D. J. Auerbach, Observation of Direct Inelastic Scattering in the Presence of Trapping-Desorption Scattering: Xe on Pt(111), Phys. Rev. Lett. 1979, 43, 1175-1177, https://doi. org/10.1103/PhysRevLett.43.1175.

[168] S. Roy, N. A. Shenvi, J. C. Tully, Model Hamiltonian for the Interaction of NO with the $\mathrm{Au}(111)$ Surface, J. Chem. Phys. 2009, 130, 174716, https://doi.org/10. 1063/1.3122989.

[169] C. Steinsiek, P. R. Shirhatti, J. Geweke, C. Bartels, A. M. Wodtke, Work Function Dependence of Vibrational Relaxation Probabilities: $\mathrm{NO}(v=2)$ Scattering from Ultrathin Metallic Films of $\mathrm{Ag} / \mathrm{Au}(111)$, J. Phys. Chem. C 2018, 122, 1002710033, https://doi.org/10.1021/acs.jpcc.8b01950. 
Bibliography

[170] B. C. Krüger, S. Meyer, A. Kandratsenka, A. M. Wodtke, T. Schäfer, Vibrational Inelasticity of Highly Vibrationally Excited $\mathrm{NO}$ on $\mathrm{Ag}(111)$, J. Phys. Chem. Lett. 2016, 7, 441-446, https://doi.org/10.1021/acs.jpclett.5b02448.

[171] I. Magoulas, A. Papakondylis, A. Mavridis, Structural Parameters of the Ground States of the Quasi-Stable Diatomic Anions $\mathrm{CO}^{-}, \mathrm{BF}^{-}$, and $\mathrm{BCl}^{-}$as Obtained by Conventional Ab Initio Methods, Int. J. Quantum Chem. 2015, 115, 771-778, https://doi.org/10.1002/qua.24899.

[172] M. W. Siegel, R. J. Celotta, J. L. Hall, J. Levine, R. A. Bennett, Molecular Photodetachment Spectrometry. I. The Electron Affinity of Nitric Oxide and the Molecular Constants of $\mathrm{NO}^{-}$, Phys. Rev. A 1972, 6, 607-631, https://doi.org/10. 1103/PhysRevA.6.607.

[173] D. R. Lide, CRC Handbook of Chemistry and Physics, 77th edition, CRC Press, Boca Raton, New York, London, Tokyo, 1996.

[174] C. S. Fadley, D. A. Shirley, Electronic Densities of States from X-Ray Photoelectron Spectroscopy, J. Res. NBS A Phys. Chem. 1970, 74, 543-558, http: //dx.doi.org/10.6028/jres.074A.045.

[175] D. P. Engelhart, R. J. V. Wagner, A. Meling, A. M. Wodtke, T. Schäfer, Temperature Programmed Desorption of Weakly Bound Adsorbates on $\mathrm{Au}(111)$, Surf. Sci. 2016, 650, 11-16, https://doi.org/10.1016/j.susc.2015.06.010.

[176] A. Kleyn, T. Horn, Rainbow Scattering from Solid Surfaces, Phys. Rep. 1991, 199, 191-230, https://doi.org/10.1016/0370-1573(91)90032-H.

[177] N. H. Nahler, J. D. White, J. LaRue, D. J. Auerbach, A. M. Wodtke, Inverse Velocity Dependence of Vibrationally Promoted Electron Emission from a Metal Surface, Science 2008, 321, 1191-1194, https://doi.org/10.1126/science.1160040.

[178] F. Grätz, D. P. Engelhart, R. J. V. Wagner, G. Meijer, A. M. Wodtke, T. Schäfer, CO $\left(\mathrm{a}^{3} \Pi\right)$ Quenching at a Metal Surface: Evidence of an Electron Transfer Mediated Mechanism, J. Chem. Phys. 2014, 141, 044712, https://doi.org/10.1063/ 1.4887777 .

[179] F. Grätz, D. P. Engelhart, R. J. V. Wagner, H. Haak, G. Meijer, A. M. Wodtke, T. Schäfer, Vibrational Enhancement of Electron Emission in CO ( $\left.\mathrm{a}^{3} \Pi\right)$ Quenching at a Clean Metal Surface, Phys. Chem. Chem. Phys. 2013, 15, 14951-14955, https://doi.org/10.1039/c3cp52468j. 


\section{Acknowledgments}

First, I would like to thank my thesis advisor Prof. Dr. Alec M. Wodtke, who offered me an excellent position for my doctoral research. I joined his group at a special time when more and more machines started to produce data three years after Alec and his group moved from Santa Barbara to Göttingen. I was immediately infected with the great enthusiasm and motivation of that time. It is not only the excellent research conditions that make me feel grateful to be part of Alec's group, but also the friendly and welcoming atmosphere in his group. I thank Alec for fruitful discussions in his office and for helpful ideas and motivation at any time.

I am indebted to Prof. Dr. Dirk Schwarzer, who kindly agreed to be the second reviewer of this thesis. Dirk put a lot of effort into the construction of a new nozzle that was implemented in two different surface scattering machines that I used.

Special thanks go to Dr. Tim Schäfer, who has been the project leader and my supervisor for several years. His excellent scientific expertise and practical skills paired with his positive attitude and his relentless enthusiasm make him an incredible person and wonderful colleague. Together, we spent many hours in the lab turning knobs, switching triggers, and watching oscilloscopes, which I will always remember when listening to songs by Cesária Évora or Sixto Rodriguez. I will also remember Tim's commitment in organizing group activities, such as the skiing trips to the Harz mountains, the sailing trip on the Baltic Sea, the pig roasting on the lawn in front of the institute on warm summer nights, and the gigs of our band "The Swinging Alectrons". I am very thankful that I met Tim and that I had the chance to learn from him in many ways.

I would like to thank Dr. Fabian Grätz and Dr. Daniel P. Engelhart. I joined their lab at a time when they were doing their PhDs. Fabian and Dan taught me basically everything that I needed to know in order to run a modern surface science lab. "Now, you're a man!" is what Dan said when he patted my shoulder after I had tightened my first CF flange. Fabian and Dan also taught me the use of the Stark decelerator, which is an astonishing and fascinating device. Many thanks go to Dr. Bastian C. Krüger, who instructed me in the use of "his" surface scattering apparatus. Bastian is a talented scientist no matter whether it comes to practical work in the lab, the analysis of data, or teaching. I am grateful to Bastian for his support including the numerous discussions 


\section{Acknowledgments}

we had and also for the great time we had at the table soccer table and during our hikes in the Hessische Schweiz or on the Hoher Meißner. I would like to thank Dr. G. Barratt Park, who helped me a lot with the understanding of quantum mechanics and spectroscopy. Barratt is always up for discussions and his extraordinarily structured and logical way of thinking makes the discussions extremely fruitful and enjoyable.

I kindly thank Dr. Tim Schäfer and Dr. G. Barratt Park for proofreading this thesis.

I thank Artur Meling, who joined my lab for his Master's thesis. Together, we struggled for months with the generation of Stark decelerated beams of high- $v$ CO, and finally succeeded. I thank Niklas Henning, who did his Master's thesis on the scattering of $\mathrm{CO}(v=17)$ from gold. Niklas knows what it means to make an experiment work that includes a total of 10 lasers. Once everything was working, he kept company and stayed late with me in the lab collecting data, or he invited me to his place where he had prepared lunch or dinner. I thank Niklas for his continuous support that goes far beyond his contributions as a master student.

Many thanks go to the whole group which includes more than 40 people. Everybody is open for discussion and lends a hand when help is needed in the lab. I appreciate the unlimited willingness to learn about and to discuss the newest results of each member of the group during our weekly group seminars and the annual group retreats at Schloß Ringberg. It is not only the dialogue and scientific spirit, but also the cheerful and relaxed atmosphere that keep up the high level of motivation and inspiration. I appreciate the collective head-scratching while puzzling over scientific problems as well as the tough table soccer matches after lunch, the cake breaks in the afternoon, the road bike trips in the evening, and the pub trips at night. Especially, I am happy about our recent trip to Sri Lanka where we visited our colleague D. M. Rasika E. A. Dissanayake, which was a great experience.

I appreciate any kind of support by the IPC manager Dr. Markus Hold, by the secretaries Jessica Paulsen, Kerstin Quentin, and Marion Diegmann, by Andreas Knorr and the whole electronics workshop, by Volker Meyer, Mike Zippert, and the whole mechanics workshop, by Bernd Wichmann, and by the IPC technical staff Clemens Heymann, Michael Schlote, Werner Noack alias Milo, and Norbert Neisen.

Many thanks go to my friends Martin Stubenvoll, Niklas Henning, Daniel Fetzer, Jan Brondke, and Dr. Ingmar Polenz (and many more) for lots of joyful moments that enriched my life and for your company when I was brooding over my $\mathrm{PhD}$ or any other task in life.

Finally, I would like to thank my parents Ursula and Reinhard Wagner. You were always there with your overwhelming patience, experience, and love. This work is dedicated to both of you. 


\section{List of Publications}

1. R. J. V. Wagner, B. C. Krüger, G. B. Park, M. Wallrabe, A. M. Wodtke, T. Schäfer, Electron Transfer Mediates Vibrational Relaxation of CO in Collisions with $\operatorname{Ag}(111)$, Phys. Chem. Chem. Phys. 2019, 21, 1650-1655, https://doi.org/ 10.1039/c8cp06041j.

2. R. J. V. Wagner, N. Henning, B. C. Krüger, G. B. Park, J. Altschäffel, A. Kandratsenka, A. M. Wodtke, T. Schäfer, Vibrational Relaxation of Highly Vibrationally Excited CO Scattered from $\mathrm{Au}(111)$ : Evidence for $\mathrm{CO}^{-}$Formation, J. Phys. Chem. Lett. 2017, 8, 4887-4892, https://doi.org/10.1021/acs.jpclett.7b02207.

3. B. C. Krüger, G. B. Park, S. Meyer, R. J. V. Wagner, A. M. Wodtke, T. Schäfer, Trapping-Desorption and Direct-Scattering of Formaldehyde at $\mathrm{Au}(111)$, Phys. Chem. Chem. Phys. 2017, 19, 19896-19903, https://doi.org/10.1039/c7cp03907g.

4. D. P. Engelhart, R. J. V. Wagner, A. Meling, A. M. Wodtke, T. Schäfer, Temperature Programmed Desorption of Weakly Bound Adsorbates on $\mathrm{Au}(111)$, Surf. Sci. 2016, 650, 11-16, http://dx.doi.org/10.1016/j.susc.2015.06.010.

5. D. P. Engelhart, R. J. V. Wagner, P. C. Johnsen, A. M. Wodtke, T. Schäfer, Adsorbate Enhancement of Electron Emission During the Quenching of Metastable CO at Metal Surfaces, Phys. Chem. Chem. Phys. 2015, 17, 11540-11545, https://doi.org/10.1039/c5cp01255d.

6. D. P. Engelhart, F. Grätz, R. J. V. Wagner, H. Haak, G. Meijer, A. M. Wodtke, T. Schäfer, A New Stark Decelerator Based Surface Scattering Instrument for Studying Energy Transfer at the Gas-Surface Interface, Rev. Sci. Instrum. 2015, 86, 043306, https://doi.org/10.1063/1.4918797.

7. F. Grätz, D. P. Engelhart, R. J. V. Wagner, G. Meijer, A. M. Wodtke, T. Schäfer, CO $\left(\mathrm{a}^{3} \Pi\right)$ Quenching at a Metal Surface: Evidence of an Electron Transfer Mediated Mechanism, J. Chem. Phys. 2014, 141, 044712, https://doi.org/10.1063/ 1.4887777 . 
List of Publications

8. F. Grätz, D. P. Engelhart, R. J. V. Wagner, H. Haak, G. Meijer, A. M. Wodtke, T. Schäfer, Vibrational Enhancement of Electron Emission in CO ( $\left.\mathrm{a}^{3} \Pi\right)$ Quenching at a Clean Metal Surface, Phys. Chem. Chem. Phys. 2013, 15, 14951-14955, https://doi.org/10.1039/c3cp52468j. 